\title{
Biogenesis of Lipid Bodies in Lobosphaera incisa
}

\author{
Dissertation \\ for the award of the degree "Doctor rerum naturalium" \\ of the Georg-August-Universität Göttingen \\ within the doctoral program GGNB Microbiology and Biochemistry \\ of the Georg-August University School of Science (GAUSS)
}

submitted by

\section{Heike Siegler}

from Münster

Göttingen 2016 



\section{Members of the Thesis Committee}

Prof. Dr. Ivo Feußner

Department for Plant Biochemistry, Albrecht-von-Haller Institute for Plant Sciences, University of Göttingen

Prof. Dr. Volker Lipka

Department of Plant Cell Biology, Albrecht-von-Haller Institute for Plant Sciences, University of Göttingen

Prof. Dr. Thomas Friedl

Department of Experimental Phycology and Culture Collection of Algae at the University of Göttingen, Albrecht-von-Haller Institute for Plant Sciences, University of Göttingen

\section{Members of the Examination Board}

Prof. Dr. Ivo Feußner (Referee)

Department for Plant Biochemistry, Albrecht-von-Haller Institute for Plant Sciences, University of Göttingen

Prof. Dr. Volker Lipka (2 ${ }^{\text {nd }}$ Referee)

Department of Plant Cell Biology, Albrecht-von-Haller Institute for Plant Sciences, University of Göttingen

Prof. Dr. Thomas Friedl

Department of Experimental Phycology and Culture Collection of Algae at the University of Göttingen, Albrecht-von-Haller Institute for Plant Sciences, University of Göttingen

Prof. Dr. Andrea Polle

Department of Forest Botany and Tree Physiology, Büsgen Institute, University of Göttingen

PD Dr. Thomas Teichmann

Department of Plant Cell Biology, Albrecht-von-Haller Institute for Plant Sciences, University of Göttingen

Dr. Martin Fulda

Department for Plant Biochemistry, Albrecht-von-Haller Institute for Plant Sciences, University of Göttingen

Date of oral examination: 30.05.2016 


\section{Affidavit}

I hereby declare that I wrote the present dissertation on my own and with no other sources and aids than quoted.

Heike Siegler

Göttingen, February 2017 


\section{Index}

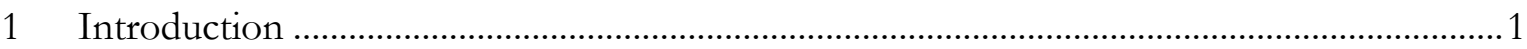

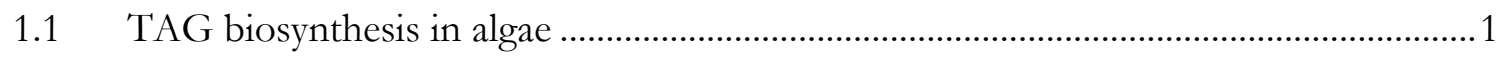

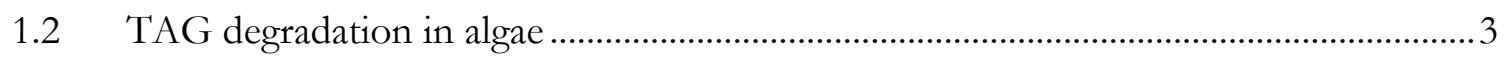

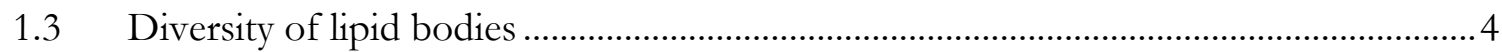

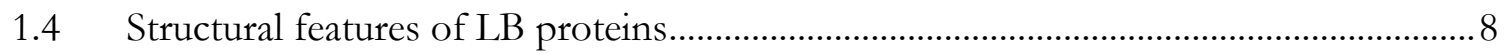

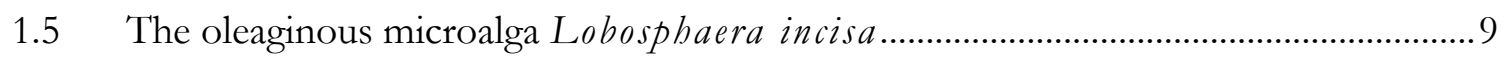

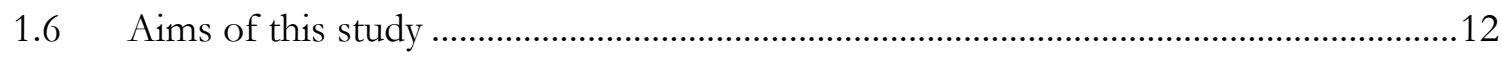

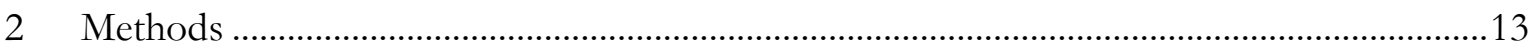

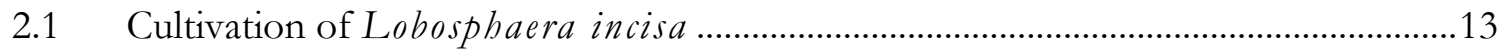

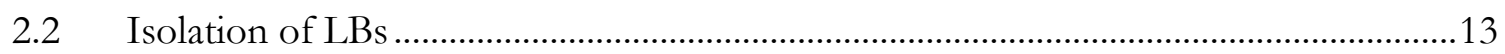

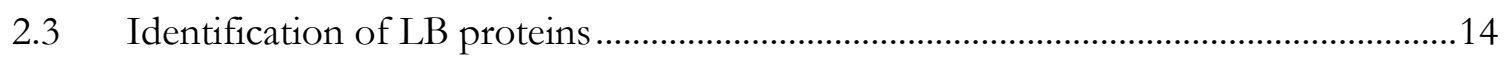

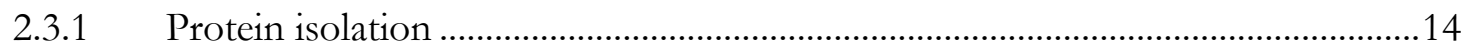

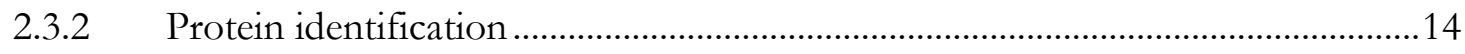

2.3.3 Enrichment analysis of identified proteins.............................................................. 16

2.3.4 In silico analyses of putative LB proteins ...........................................................17

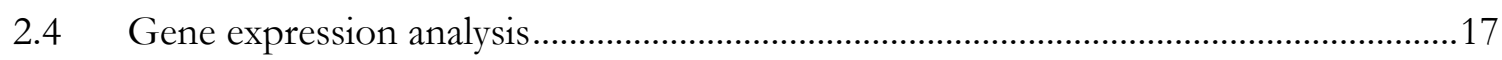

2.4.1 L. incisa growth under conditions of nitrogen starvation and recovery.............17

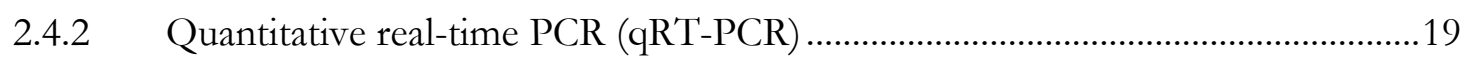

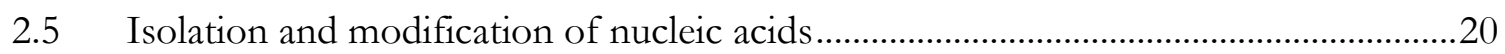

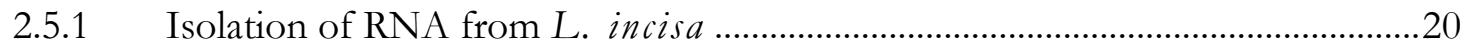

2.5.2 Isolation of RNA from Arabidopsis thaliana seeds ............................................20

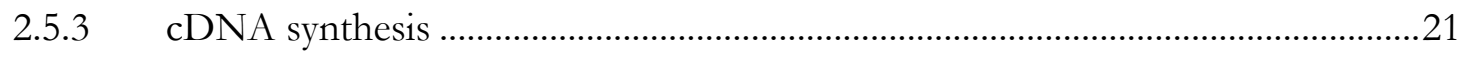

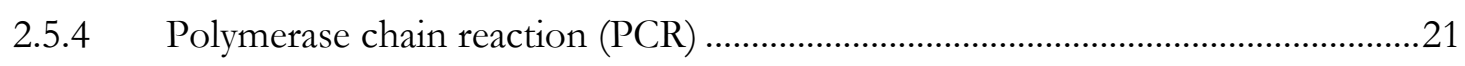

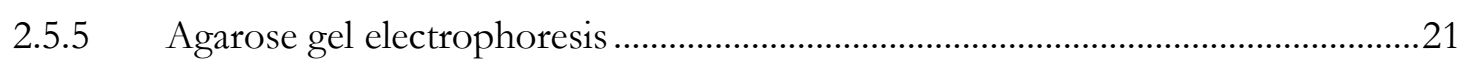




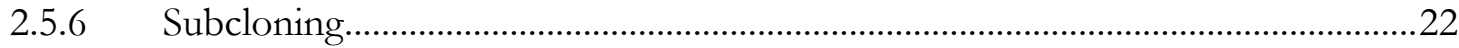

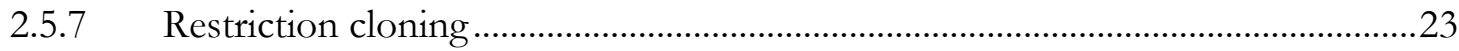

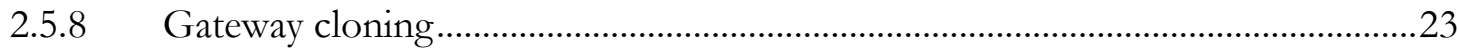

2.5.9 Generation of chemically competent bacteria …....................................................2

2.6 Transient gene expression in Nicotiana tabacum pollen tubes ...................................24

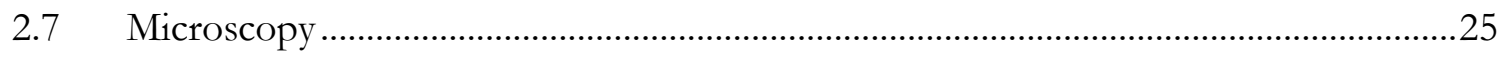

2.7.1 Detection of neutral lipids in L. incis a cells and isolated LBs............................25

2.7.2 Localization studies in N. tabacum pollen tubes ..................................................26

2.7.3 Selection of Arabidopsis thaliana seeds expressing the $m$ Cherry gene ...........26

2.8 Complementation of Arabidopsis thaliana mutants....................................................2

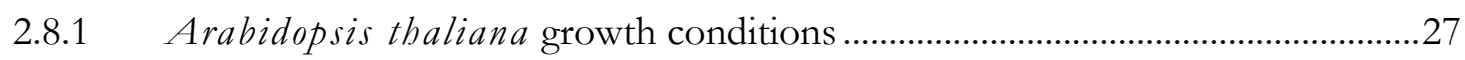

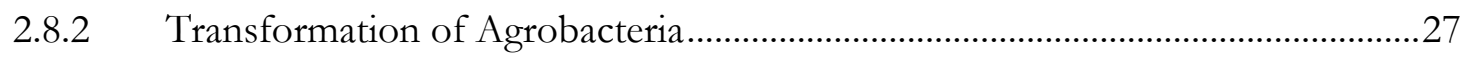

2.8.3 Agrobacterium-mediated transformation of Arabidopsis thaliana ....................28

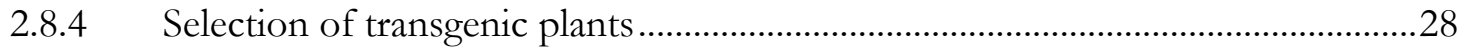

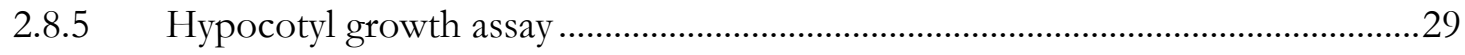

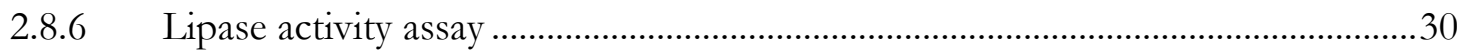

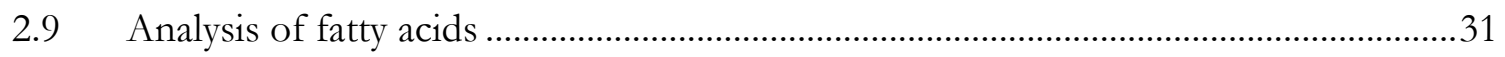

2.9.1 Analysis of fatty acids in Arabidopsis thaliana seeds and seedlings ..................31

2.9.2 Analysis of fatty acids in TAG and total extracts of $L$. incisa …...........................32

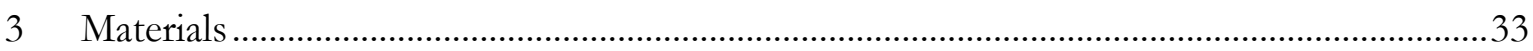

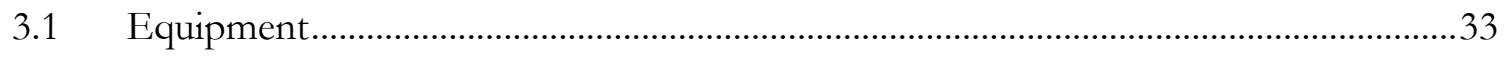

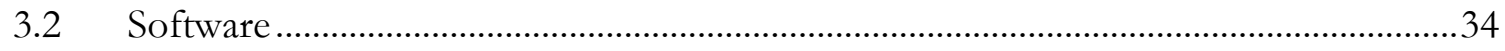

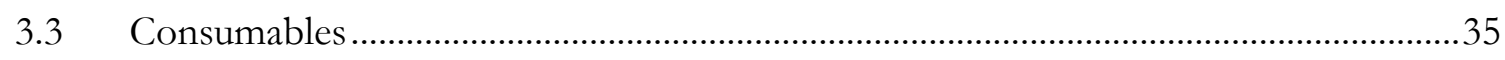

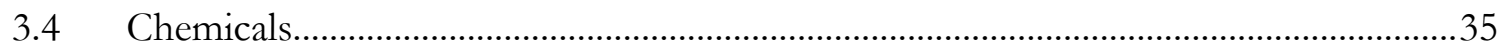

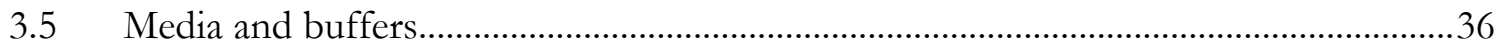

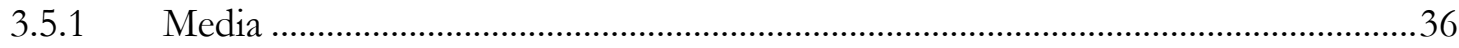




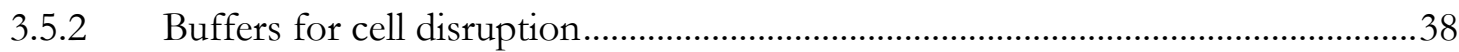

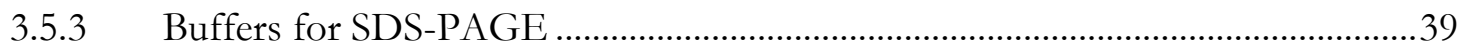

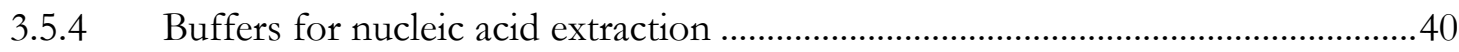

3.5.5 Buffers for agarose gel electrophoresis ..................................................................41

3.5.6 TFP buffer for generation of competent $E$. coli ...................................................... 41

3.5.7 Tris EDTA buffer (TE buffer) for Gateway cloning .............................................42

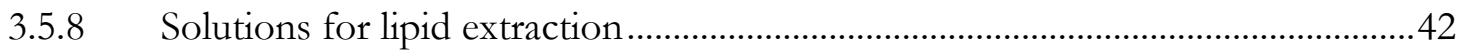

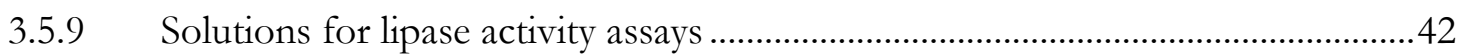

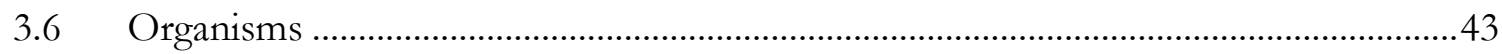

3.6.1 Algae and plant lines..........................................................................................

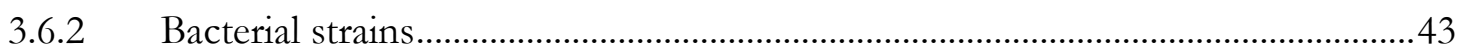

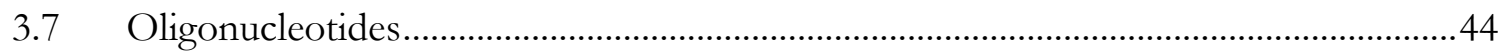

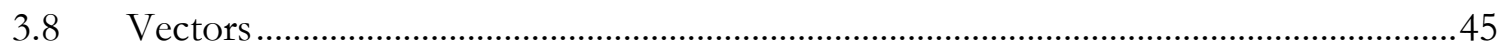

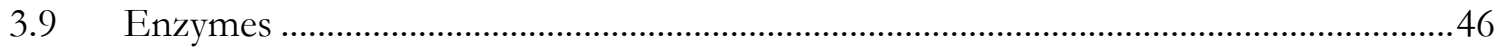

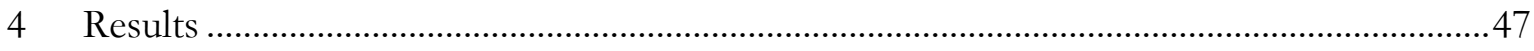

4.1 Analysis of physiological responses to changes in nitrogen availability …....................47

4.1.1 Determination of pigment and fatty acid content.................................................4

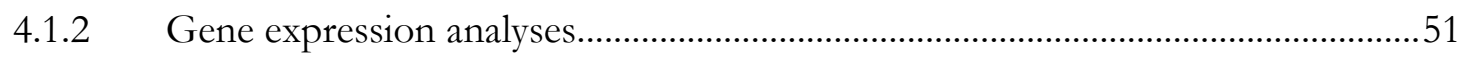

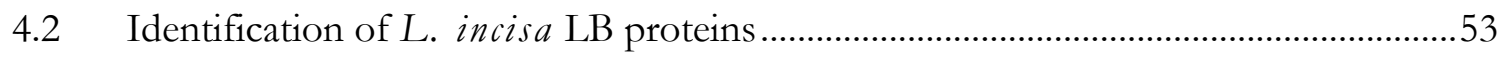

4.2.1 Characterization of L. incisa OIL GLOBULE PROTEIN (LiOGP) ................56

4.2.2 Characterization of the L. incisa g15430 protein ................................................59

4.2.3 Characterization of the L. incisa g13747 protein ................................................63

4.2.4 Characterization of the L. incisa g13945 protein ..................................................65

4.3 Identification of enzymes involved in triacylglycerol degradation ..................................71

4.3.1 Characterization of the putative lipase g2905 _.................................................. 
4.3.2 Characterization of the L. incisa SUGAR-DEPENDENT 1 lipase (LiSDP1)

5 Discussion...

5.1 Lobosphaera incis a physiology is strongly affected by nitrogen supply....

5.2 Nitrogen supply affects expression of genes commonly used for normalization in qRT-PCR...

5.3 Several LB proteins were identified in this study ......................................................... 90

5.3.1 LiOGP, g15430 and g13747 are LB associated proteins of unknown function . .92

5.3.2 g13945 is an LB-localized putative lipase ................................................................ 93

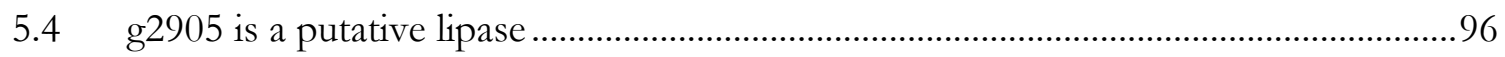

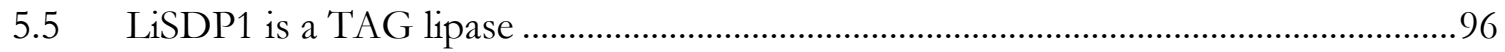

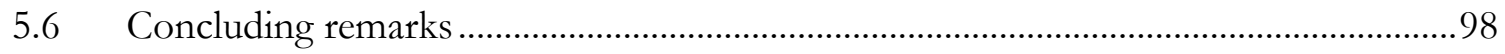

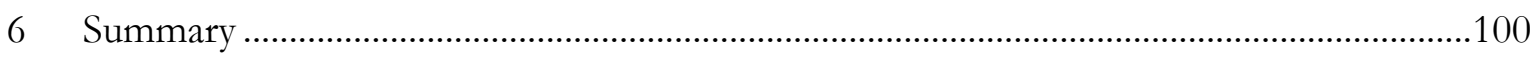

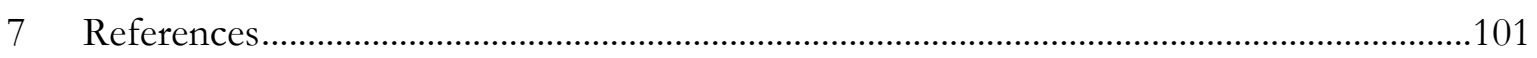

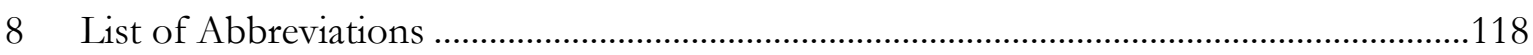

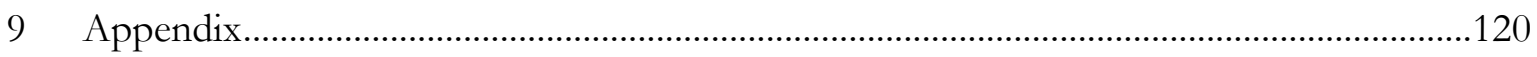

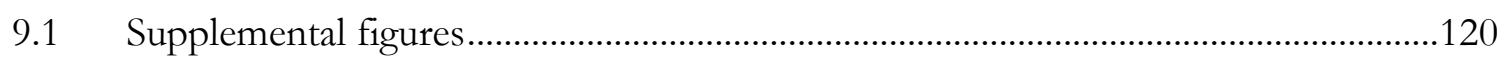

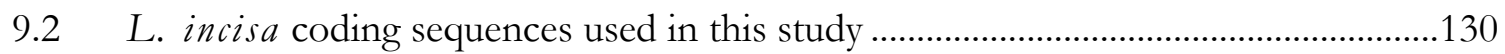

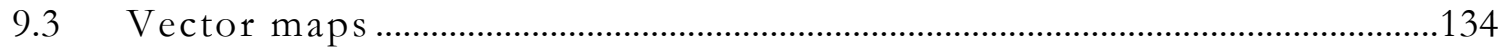

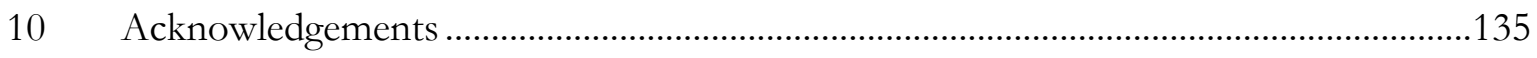

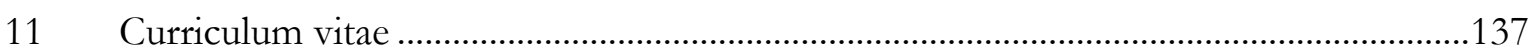




\section{Introduction}

Microalgae are a highly heterogeneous group of organisms that can be found in diverse habitats ranging from snowy mountains to the deep sea. Their fundamental role in the respective ecosystems has been thoroughly studied and the elucidation of cellular processes such as photosynthesis has been greatly aided by the characterization of microalgae on a molecular scale. Some microalgal species accumulate high levels of the neutral lipid triacylglycerol (TAG) and store it within the cell as carbon and energy reserve. They have therefore gained attention as possible producers of biofuels (Shi et al., 2011) or high value products such as polyunsaturated fatty acids (PUFAs) (Khozin-Goldberg et al., 2011). Cultures of these oleaginous algae can be stimulated to upregulate TAG synthesis by exposing them to a range of abiotic stresses, deprivation of nitrogen being most effective in this respect. This stress response is accompanied by far-reaching changes in overall metabolism, including growth arrest (Cheng-Wu et al., 2002; Davidi et al., 2012; Msanne et al., 2012; Tsai et al., 2014), a drastic downregulation of photosynthesis on a transcript level (Miller et al., 2010; Schmollinger et al., 2014), degradation of chloroplasts (Allen et al., 2015; Davidi et al., 2012; Msanne et al., 2012; Peled et al., 2011; Schmollinger et al., 2014) and a restructuring of the proteome towards a lower nitrogen content (Schmollinger et al., 2014). Remarkably, these extensive adjustments are reversible by resupplying nitrogen to the growth media (Khozin-Goldberg et al., 2005; Tsai et al., 2014). Keeping in mind the potential application of TAG derived products, the fact that oleaginous microalgae are this responsive to manipulation makes them especially interesting for the study of lipid metabolism.

\subsection{TAG biosynthesis in algae}

TAG is a highly reduced molecule consisting of three acyl chains that are esterified to a glycerol backbone. A large part of our knowledge of algal TAG synthesis was initially derived from insights gained in plant research by inferring the function of many algal genes from homology with plant counterparts that had already been characterized as parts of lipid metabolism (Liu and Benning, 2013; Moellering et al., 2009). When the functions of numerous proteins encoded in the algal genomes were validated through reverse genetic studies, many similarities in fundamental processes leading up to the accumulation of TAG were confirmed and some features unique to algae were uncovered (Zienkiewicz et al., 2016). 
The assembly of acyl lipids requires fatty acids, which are synthesized in the chloroplast by the fatty acid synthase complex. Using pyruvate-derived malonyl-ACYL CARRIER PROTEIN (malonyl-ACP) as a building block, this enzyme complex generates 16:0 and 18:0 fatty acids bound to ACP through repeated cycles of condensation, reduction, dehydration and reduction as well as 18:1-ACP through a subsequent desaturation step. In Arabidopsis thaliana leaves, the majority of fatty acids is exported from the plastid following cleavage of ACP and is activated by addition of Coenzyme A (CoA). Acyl-CoAs can be used for the synthesis of glycerolipids via the Kennedy pathway at the endoplasmic reticulum (ER), which begins with the sequential addition of the acyl moieties to the $s n-1$ and $s n-2$ positions of glycerol-3-phosphate by acyl-CoA:glycerol-3-phosphate acyltransferase (GPAT) and acyl-CoA:lysophosphatidic acid acyltransferase (LPAAT), respectively, resulting in phosphatidic acid. This molecule is subsequently dephosphorylated by phosphatidic acid phosphatase (PAP), forming diacylglycerol (DAG). DAG can then be used for the synthesis of membrane glycerolipids through the addition of a polar headgroup, or acylated at the $s n-3$ position to yield the neutral lipid TAG. This final step can be catalyzed by 2 different enzyme families that have been characterized in algae so far and which differ in the type of lipid they use as an acyl donor. Firstly, acyl-CoA:diacylglycerol acyltransferase (DGAT) enzymes catalyze the esterification of a fatty acid from acyl-CoA to DAG. Three types of DGAT, differing strongly in amino acid sequence and subcellular localization, have been identified in plants and two of them have been functionally characterized in algae. Secondly, the final acyl group for TAG synthesis can be transferred from membrane lipids to DAG by phospholipid:diacylglycerol acyltransferase (PDAT). This enzyme has been shown to contribute to TAG biosynthesis in C. reinhardtii, transferring acyl groups not only from phospholipids, as its plant homologs (Dahlqvist et al., 2000; Stahl et al., 2004), but also from other lipid subclasses including DAG and galactolipids (Yoon et al., 2012).

In plants, approximately $40 \%$ of fatty acids remain in the chloroplast and enter a "prokaryotic pathway" of lipid synthesis with high similarity to the extraplastidic Kennedy pathway. The subsequent addition of polar headgroups results in the formation of constituents of thylakoid and envelope membranes such as monogalactosyldiacylglycerol (MGDG). Plant lipids originating from the chloroplast may be distinguished from those derived from the ER by the acyl chain occupying the $s n-2$ position, as the LPAAT in the chloroplast exhibits a strong preference for 16:0, whereas the isozyme at the ER membrane preferentially incorporates 18 
carbon fatty acids into lysophosphatidic acid. The observation that most TAG molecules in C. reinhardtii carry a 16:0 fatty acid in this position hints at a possible plastidial origin of the storage lipid, presuming the $s n$-specificity of the LPAAT isoforms holds true for algae, which has not been demonstrated to date.

\subsection{TAG degradation in algae}

Rescuing microalgae after a phase of stress induces an adaptation of the overall metabolism, which undergoes a shift away from the accumulation of storage lipids towards growth fueled by photosynthesis. For this purpose, chloroplasts must be rebuilt, a process that can be quantified in terms of increased galactolipid and chlorophyll synthesis. Fatty acids from TAG are used as a source of carbon and energy for this drastic remodeling of cellular functions. This requires the action of TAG- DAG- and monoacylglycerol (MAG) lipases to cleave off fatty acids, which can then be degraded through $\beta$-oxidation. An enzyme with the latter two lipase activities has been characterized in C. reinhardtii (LIPASE 1, CrLIP1) and appears to be linked to TAG hydrolysis, as reduced gene expression leads to a delay in TAG mobilization (Li et al., 2012b). However, in contrast to plants, mammals or other microorganisms, a lipase catalyzing the first step in the mobilization of TAG has not been identified in C. reinhardtii or any other microalga, with the exception of the diatom $P$. tricornutum. In this organism, a protein belonging to the family of patatin domain lipases has recently been described (TAG LIPASE 1, TGL1, Barka et al., 2016). This family of enzymes is named after a phospholipase A originally described in potato tubers (Andrews et al., 1988) and also comprises diverse lipases including the major TAG lipases in A. thaliana seedlings (SUGAR DEPENDENT 1, AtSDP1, Eastmond, 2006) and Saccharomyces cerevisiae (ScTGL3-5, Athenstaedt and Daum, 2003, 2005). So far, the assumption of TAG lipase activity for $P$. tricornutum TGL1 is based on the recombinant protein exhibiting esterase activity on the substrate analog para-nitrophenyl butyrate as well as on increased TAG accumulation in knockdown mutants (Barka et al., 2016). A study directly implicating the enzyme in the first step of TAG hydrolysis rather than subsequent degradation of DAG and MAG as demonstrated for CrLIP1 has not been published to date.

While the enzymes that are directly involved in algal TAG metabolism are being characterized one by one, only very little information has been gathered on their transcriptional regulation. In C. reinhardtii, a putative transcription factor specifically involved in the response to nitrogen 
starvation (Boyle et al., 2012) and a kinase that possibly regulates other transcription factors in nitrogen- and sulfur deplete conditions (Kajikawa et al., 2015) have been identified but not characterized in more detail.

Overall, despite vast overlaps between the features of acyl lipid metabolism in plants and algae, there seem to be differences. In addition to the discrepancies mentioned above, a high degree of variation can be found in the copy numbers of genes encoding acyltransferases of the Kennedy pathway between microalgae and plants as well as among algal species (Zienkiewicz et al., 2016). This observation indicates that the degree of complexity in glycerolipid synthesis may not be the same in all of these organisms, a notion that is supported by the fact that C. reinhardtii lacks phosphatidylcholine (PC), in contrast to plants and many other algae (Giroud et al., 1988).

\subsection{Diversity of lipid bodies}

TAG is an unpolar lipid molecule and therefore cannot be a constitutive part of a membrane, nevertheless it needs to be stored in a way that allows mobilization when necessary. This function is fulfilled by cytosolic lipid bodies (LBs). Early studies on the composition of LBs were carried out in onion, cabbage and cottonseed (Yatsu et al., 1971), while their structure was first elucidated in peanut seedlings (Yatsu and Jacks, 1972). Since then, LBs have been found to occur in an astonishing range of organisms, tissues, developmental stages and environmental conditions. They can measure between $50 \mathrm{~nm}$ (in milk-secreting cells) and $200 \mu \mathrm{m}$ (in mature adipocytes) in diameter (Murphy, 2001) and are generally composed of a core of neutral lipids surrounded by a monolayer of polar lipids with proteins directly or indirectly attached to the surface (Yatsu and Jacks, 1972).

In mammals, LBs are being increasingly investigated as their relevance for human health and disease becomes more and more clear. They have been found in a large variety of cell types, where they are thought to fulfill a range of functions that are connected to the presence of different proteins on the LB surface. Adipocytes are naturally abundant in LBs, which carry enzymes involved in lipid metabolism and lipid secretion on their surface (Brasaemle et al., 2004; Love et al., 2015). LBs in hepatocytes carry adipose TAG lipase on their surface (Eichmann et al., 2015) among other proteins that are thought to be involved in fatty liver diseases (Carr and Ahima, 2015). Activated mast cells (Dichlberger et al., 2015), a Chinese hamster ovary cell line (Bartz et al., 2007), enterocytes (Beilstein et al., 2015) and cells 
originating from the skin (Dahlhoff et al., 2015) as well as the skeletal muscle (Bosma, 2015) have equally been investigated with regards to the LB proteome. LBs and their protein components have been established to play a major role in the inflammatory response of leukocytes through the synthesis and regulation of eicosanoids, fatty acid derived mediators of inflammation (Melo and Weller, 2015). Additionally, a subset of testicular cells accumulates LBs that are rich in cholesteryl esters, which are required as precursors for testosterone synthesis (Wang et al., 2015) and LBs have even been identified within the nuclei of hepatic cells (Layerenza et al., 2013; Uzbekov and Roingeard, 2013; Wang et al., 2013). Despite this broad spectrum of tissue specific functions, all mammalian LBs that have been characterized so far possess structural proteins belonging to the perilipin (PLN) family, consisting of PLN1-5 (Sztalryd and Kimmel, 2014), which regulate neutral lipid degradation by lipases (Sztalryd and Kimmel, 2014; Wang et al., 2011). They need to be degraded in order for lipases to be able to access their substrate (Schweiger and Zechner, 2015) and $p l n$ knockout mice are characterized by constitutive lipolysis (Martinez-Botas et al., 2000). The determinant role of PLNs in LB structural integrity is further underlined by the observation that heterologous PLN expression alone is sufficient to induce the formation of LBs in neutral lipid-enriched yeast cells (Jacquier et al., 2013; Mishra and Schneiter, 2015).

A homolog of PLNs has been identified in analyses of the Drosophila melanogaster fat body tissue LB proteome and was shown to fulfill a comparable function in the regulation of LB degradation (Beller, 2006; Grönke et al., 2003; Teixeira et al., 2003). In vitro experiments with insect cells have shown that GPAT4, the first enzyme in the Kennedy pathway, relocalizes from the ER to a subset of LBs that then grow upon addition of exogenous fatty acid (Wilfling et al., 2013), an aspect of LB proteome dynamics that appears to be conserved in mammals (Wilfling et al., 2013). In addition to merely storing carbon and energy in the form of neutral lipids, certain D. melanogaster stem cell LBs seem to have a role in protecting membranes during oxidative stress by sequestering PUFAs, thus protecting them from harmful peroxidation (Bailey et al., 2015).

Not only mammals and insects have been the subject of studies focusing on LBs, but unicellular organisms are also of great interest regarding the composition, metabolism and function of this organelle where no specialized tissues exist. For many years, LBs in S. cerevisiae have been studied and in contrast to other oleaginous organisms known so far, yeast LBs contain a large amount of sterol esters, comprising approximately half of the neutral lipids, the other half being 
TAG (Clausen et al., 1974). Both classes of neutral lipid serve as sources of acyl moieties for membrane lipid synthesis (Daum and Paltauf, 1980). In contrast to other organisms, no structural LB proteins have so far been identified in $S$. cerevisiae, but a number of other proteins has been reported to be associated with the LB surface (Athenstaedt et al., 1999; Grillitsch et al., 2011). TAG and sterol biosynthesis appear to take place at the LB as indicated by the presence of a DGAT (Oelkers et al., 2002; Sorger and Daum, 2002) and a sterol $\Delta^{24}$-methyltransferase (Zinser $e$ t al., 1993), as well as TAG degradation by TAG LIPASEs 3-5 (TGL 3-5) (Athenstaedt and Daum, 2003, 2005).

Plants have been studied intensively for their lipid metabolism, in part motivated by potential applications in the production of oil for food, feed and industrial applications. In this context, A. thaliana has become a very useful model organism to study TAG metabolism in oilseeds and the findings made in analyses of seed LB protein composition in this organism (Jolivet et al., 2004; Vermachova et al., 2011) have been confirmed as well as complemented with studies in crop plants such as rapeseed (Brassica napus) (Jolivet et al., 2009; Katavic et al., 2006), maize (Zea mays) (Tnani et al., 2011), sesame (Sesamum indicum L.) (Chen et al., 1998), sunflower (Helianthus annuus L.) (Thakur and Bhatla, 2016), peanut (Arachis bypogea) (Jolivet et al., 2013), cucumber (Cucumis sativus) (Sturm et al., 1985) and false flax (Camelina sativa) (Jolivet et al., 2013).

In all oilseed LB isolations so far, the most abundant protein was oleosin, originally identified in maize seeds (Vance and Huang, 1987). 16 members of this protein family are encoded in the A. thaliana genome, of which 5 are specifically expressed in maturing seeds (Kim et al., 2002). These proteins are now known to play an important role in the structural integrity of LBs, as a reduction in oleosin abundance drastically increases LB size in seeds, which has been correlated with a delay in germination (Siloto et al., 2006). Oleosin degradation is required for LB breakdown (Deruyffelaere et al., 2015) and the proteins have been assigned with a function in the freezing tolerance of seeds (Shimada et al., 2008). An additional protein that is frequently found associated with seed LBs is caleosin (Chen et al., 1998). The function of this protein, named after its ability to bind calcium and the structural similarity with oleosins (Chen et al., 1999), has not been exhaustively studied. It has been assigned peroxygenase activity in oat seeds (Hanano et al., 2006) and a function in vacuolar degradation of LBs in A. thaliana and B. napus (Poxleitner et al., 2006), while in $A$. thaliana seeds a role the in abiotic stress response has also been postulated (Takahashi et al., 2000). A third protein that is frequently 
found at the surface of plant seed LBs and bears structural resemblance to oleosins is steroleosin (Lin et al., 2002). It exhibits hydroxysteroid dehydrogenase activity (d'Andréa et al., 2007) and based on overexpression and knockdown studies, a role in brassinosteroid-mediated signaling has been proposed for this protein (Baud et al., 2009; Li et al., 2007), however the exact nature of its role remains unclear. Other enzymatic activities at seed LBs have been more clearly characterized, such as a lipoxygenase (LOX) that acts on both TAG and the polar lipid surface of the LB in cucumber seedlings, thereby contributing to LB degradation during germination (Feussner and Kindl, 1992; Feussner et al., 1995; Sturm et al., 1985). Furthermore, the major TAG lipase in A. thaliana seed germination, SDP1, is an LB protein (Eastmond, 2006). AtSDP1 transcripts accumulate during seed maturation and the protein is responsible for bulk TAG degradation during postgerminative growth of the seedling (Eastmond, 2006), a function that is supported by its close homolog SDP1-LIKE (SDP1-L) (Kelly et al., 2011). Yet another TAG lipase is associated with LBs in castor bean (Ricinus communis) seeds (OIL BODY LIPASE 1, OBL1) (Eastmond, 2004). It has been classified as an acid lipase as it is most active at pH 4 (Eastmond, 2004), however its physiological role has not been elucidated so far. Furthermore, the activity of a phospholipase at the LBs in cucumber seedlings is thought to facilitate access to the core of the organelle for TAG degrading enzymes (Rudolph et al., 2011). LB associated proteins are not only important for germination processes in oilseeds, but also in pollen, although substantially less research has been carried out on LBs in reproductive tissues. The TAG lipase SDP1-L is more highly expressed in mature pollen than in any other A. thaliana tissue (Kelly et al., 2011), however it has not been shown whether the protein localizes to the LBs in this tissue and fulfills a function analogous to its homolog. In analogy to seeds, oleosins have also been reported to associate with the LB surface in developing pollen (Kim et al., 2002) as well as the floret tapetum (Kim et al., 2002; Lévesque-Lemay et al., 2015). The fruit tissues of olive, avocado and oil palm are also abundant in LBs, however compared to seed LBs these are substantially larger and do not contain oleosins (Murphy, 2001). Instead, they harbor different structural surface proteins, which are termed LIPID DROPLET ASSOCIATED PROTEINs (LDAPs, Horn $e t$ al., 2013) and do not resemble any known LB proteins in other organisms.

Although the majority of TAG accumulation in plants takes place in the tissues mentioned above, LBs have also been detected in vegetative tissues. In $A$. thaliana sdp1 roots for instance, considerable amounts of LBs accumulate (Kelly et al., 2013) and LBs have equally 
been reported for leaf mesophyll as well as leaf epidermal tissues (Pautov et al., 2016; Shimada and Hara-Nishimura, 2015).

Microalgal LBs are being characterized in a rapidly increasing number of species and parallels with plant LBs can be observed, however some striking differences have been revealed. Firstly, the accumulation of high levels of TAG is a stress response in most algae studied to date, as opposed to a part of regular developmental processes. Secondly, oleosins and perilipins are absent from all algal species that have been investigated. Instead, a family of proteins that forms a new clade of structural LB proteins seems to take over this function in many unicellular algal species. The MAJOR LIPID DROPLET PROTEIN (MLDP) of C. reinhardtii was the first one of these to be identified (Moellering and Benning, 2010) and expression of the gene has been used as a marker for TAG accumulation in C. reinhardtii (Tsai et al., 2014). CrMLDP has been shown to recruit other proteins, especially tubulins, to the LBs during nitrogen starvation. Homologs have since been characterized in Nannochloropsis (LIPID DROPLET SURFACE PROTEIN, LDSP) (Vieler et al., 2012), Haematococcus pluvialis (OIL GLOBULE PROTEIN, OGP) (Peled et al., 2011) and Dunaliella salina (MLDP) (Davidi et al., 2012), while homologous genes are present in the genomes of further microalgae of the Volvocales and Chlorellales order (Davidi et al., 2012). The most extensively characterized diatom, the oleaginous alga P. tricornutum, has been found to possess yet another type of structural LB protein that is different from all other LB proteins described above (Yoneda $e$ t al., 2016). Caleosin or steroleosin homologs have not been reported for any algal species, with the exception of a caleosin-like protein of unknown function in Chlorella (Lin et al., 2012) and Auxenochlorella protothecoides (Pasaribu et al., 2014).

Apart from these highly diverse structural proteins, algal LB proteomes also include enzymes with a broad range of predicted functions in lipid metabolism and other processes, as reflected in comprehensive datasets obtained in studies of C. reinhardtii (Moellering and Benning, 2010; Nguyen et al., 2011) and Dunaliella bardawil (Davidi et al., 2015).

\subsection{Structural features of LB proteins}

Oleosins, constituting the most intensively studied plant structural LB protein family in plants, are relatively small proteins $(15-30 \mathrm{kDa}$, Chapman et al., 2012) and possess three features permitting them a direct and strong association with LBs (Tzen et al., 1992). Firstly, a prominent sequence of hydrophobic residues allows the protein to become an integral part of 
the LB surface. Caleosins and steroleosins harbor a similar domain, as do Nannochloropsis LDSP and the $P$. tricornutum structural LB protein, thus pointing to a functional conservation of this type of domain even in the absence of sequence homology. Secondly, a motif termed "proline knot" that consists of three proline residues within a sequence of 12 amino acids is located within the hydrophobic region of the protein (Abell et al., 1997). It protrudes into the TAG core of the LB and is thought to be crucial in anchoring the protein in the organelle. Interestingly, a hydrophobic domain containing this motif is also required for LB localization of Hepatitis C virus core protein in mammalian cells (Graham Hope et al., 2002). The proline knot is equally conserved in caleosins and present in modified form as a "proline knob" in steroleosins (Lin et al., 2002). Thirdly, two amphipathic helices flank the hydrophobic region of oleosins (Tzen et al., 1992). The hydrophobic and polar faces of each helix associate with the acyl moieties and the polar headgroups of membrane lipids, respectively (Segrest et al., 1974), thus allowing proteins to bind to membranes or LBs. Mammalian and insect perilipins exclusively make use of this type of structural element to bind to LBs (Najt et al., 2014; Rowe et al., 2016), however the respective regions of the protein only assume a helical conformation in the presence of a lipid surface, a property that greatly impeded the elucidation of this feature of perilipins (Rowe et al., 2016). Not all known LB proteins however possess one or more of the above-mentioned features. The cucumber LB LOX for instance is targeted to the LB by means of its $\beta$-barrel domain (May et al., 2000) and for other enzymes such as the lipases A. thaliana SDP1 and R. communis OBL1 or the structural LDAPs, the determinant for LB localization is currently unknown.

LB proteins thus form a highly diverse group of proteins in terms of both function and mechanism of LB localization. As an increasing number of oleaginous organisms is being investigated with this regard, additional information will be gained and help to understand the complex functions of LBs.

\subsection{The oleaginous microalga Lobosphaera incisa}

Model organisms have helped to elucidate many basic processes in lipid metabolism, however the expanding scope of species analyzed has shown that new organisms from different ecological and evolutionary backgrounds are valuable for the discovery of novel mechanisms. In this study, a unicellular alga (strain SAG 2468) was investigated, which was originally isolated from a glacier in Japan, tentatively classified as Myrmecia incisa but later proposed to be named 
Parietochloris incisa (Watanabe et al., 1996) and subsequently Lobosphaera incisa (Karsten et al., 2005). A closely related strain of $M$. incisa originating from the Ötztal, Austria, has retained the original classification as assigned by Reisigl (Ouyang et al., 2012; Reisigl, 1964). Both strains as well as Coccomyxa subellipsoidea strain C-169 belong to the class of Trebouxiophyceae within the phylum Chlorophyta and have been studied with regards to lipid metabolism.

Several resources and molecular biology tools are available for the algal strains mentioned above, facilitating their experimental characterization. Within the European consortium Genetic Improvement of Algae for Value Added Products (GIAVAP), the L. incisa nuclear and plastidial genomes as well as the transcriptome of cultures subjected to $0 \mathrm{~h}, 12 \mathrm{~h}$ and $72 \mathrm{~h}$ of nitrogen depletion were sequenced using Illumina technology (unpublished data, Tourasse et al., 2014, 2015). The genome of $C$. subellipsoidea and the transcriptome of $M$. incisa are equally available (Blanc et al., 2012; Ouyang et al., 2013). Out of the three strains, L. incisa is the best characterized and a protocol for its stable transformation has been established (Zorin et al., 2014), even though further optimization of this protocol is required to achieve efficient transformation for reverse genetic studies. Furthermore, the genome of this strain has been successfully altered by chemical mutagenesis resulting in a marked phenotype (Iskandarov et al., 2011), demonstrating the feasibility of knockout mutations for analyses of this organism. While the TAG accumulated by most algae that have been analyzed to date consists mostly of saturated or monounsaturated mid and long chain fatty acids (Hu et al., 2008), the L. incisa strain analyzed in this study is one of the few species in which partitioning of the very long chain PUFA arachidonic acid (20:4 (n-6), ARA) into TAG has been reported (Bigogno et al., 2002a). A higher abundance of this fatty acid has not been observed in any other plant or alga. During logarithmic growth, the alga produces TAG which then makes up $43 \%$ of TFAs, ARA being the most abundant fatty acid in this lipid class (Bigogno et al., 2002a). When the culture is deprived of nitrogen, TAG accumulates, comprising $87 \%$ of TFAs after $14 \mathrm{~d}$ and the fraction of ARA in this lipid class increases to almost 2/3 of TFAs (Khozin-Goldberg et al., 2002). In response to a temperature decrease from $25^{\circ} \mathrm{C}$ to $12{ }^{\circ} \mathrm{C}$ or even $4{ }^{\circ} \mathrm{C}$, ARA is mobilized from TAG and transferred to polar lipids (Bigogno et al., 2002b), a process that may have evolved as part of the strain's adaptation to its native glacial environment. When grown in nitrogen limiting conditions, chloroplasts are degraded and additional LBs are formed. The ratio of carotenoids and chlorophylls is altered during his process and has been demonstrated to closely 
correlate with ARA content and thus with TAG accumulation, permitting a simple way of monitoring the overall physiological state of the culture (Merzlyak et al., 2007). In a further study, the alga has been reported to respond to high light stress by depositing $\beta$-carotene in LBs (Solovchenko et al., 2008a).

Beyond these observations, several aspects of lipid metabolism in L. incisa and its close relatives have been elucidated. Biosynthesis pathways for ARA have been proposed based on pulse-chase experiments with radiolabeled acetate, demonstrating that $\Delta^{12}$ and $\Delta^{6}$ desaturation take place at fatty acids esterified to either PC or the betaine lipid diacylglyceryltrimethylhomoserine, while fatty acids need to be incorporated into PC or phosphatidylethanolamine for $\Delta^{5}$ desaturation to take place (Bigogno et al., 2002c). This possible combination of pathways is distinct from other algal species (Bigogno et al., 2002c; Nichols and Appleby, 1969), but has been in part confirmed by an analysis of expressed sequence tags in M. incisa (Ouyang et al., 2012). For both closely related strains, the C18 $\Delta^{6}$ PUFA elongase involved in this pathway has been shown to be upregulated on a transcriptional level in response to nitrogen limitation and has been characterized by heterologous expression in yeast (Iskandarov et al., 2009; Yu et al., 2012). When ARA synthesis is disrupted by a mutation in the $\Delta^{5}$ desaturase rendering it inactive, TFAs still increase during nitrogen depletion to a comparable amount as in WT cultures and 18:1 (n-9) as well as 20:3 (n-6), the substrate of the desaturase, become the major fatty acids (Iskandarov et al., 2011). The Kennedy pathway of glycerolipid synthesis has also been studied in the two strains. In L. incisa, an extraplastidial GPAT has been partially characterized by overexpression in C. reinhardtii, confirming its role as a part of the TAG biosynthesis pathway (Iskandarov et al., 2015). A DGAT1 and two DGAT2 enzymes have been identified in $M$. incisa and their function in catalyzing the last step of TAG synthesis was confirmed by heterologous expression in a neutral lipid deficient strain of $S$. cerevisiae (Chen et al., 2015). Specificity for certain fatty acids could not be observed and a predominant activity during nitrogen starvation has not been reported to date.

In summary, $L$. incisa is a promising organism for the investigation and manipulation of PUFA accumulation in algal neutral lipids. Further characterization of this organism with respect to TAG metabolism can help to elucidate in more detail the diversity of algal cellular processes. 


\subsection{Aims of this study}

The observation of LBs in a broad spectrum of cell types and conditions has led to an increasing fascination with the physiological relevance and molecular composition of these organelles. Unicellular algae are particularly interesting in this regard due to the capacity for high-level TAG and PUFA synthesis in some strains. While the mechanisms of algal TAG biosynthesis are relatively well understood owing to studies in higher plants and the model green alga C. reinhardtii, other aspects of LB biogenesis appear to be more variable between species and have not been studied as exhaustively.

The main objective of this study was to investigate the storage and degradation of neutral lipids in the ARA-accumulating unicellular green alga L. incisa. Here, the focus was primarily on proteins associating with LBs. Additionally, similarities to known model organisms were harnessed in the identification of enzymes possibly involved in LB degradation. 


\section{Methods}

All nucleotide and predicted amino acid sequences used in this study were obtained from the unpublished results of genome and RNA sequencing experiments carried out within the consortium Genetic Improvement for Value Added Products (GIAVAP) as part of the $7^{\text {th }}$ Framework Programme for Research and Development of the European Commission. See appendix 0 for coding nucleotide sequences of genes used in this study.

\subsection{Cultivation of Lobosphaera incisa}

L. incisa strain SAG 2468 was kindly provided by Dr. Inna Khozin-Goldberg, Ben-Gurion University of the Negev, Israel, and cultivated in BG11 media (Stanier et al., 1971) in $400 \mathrm{~mL}$ glass columns with an inner diameter of $3 \mathrm{~cm}$ (Ochs $\mathrm{GmbH}$, Bovenden, Germany). Volumes of $300 \mathrm{~mL}$ were kept at $25^{\circ} \mathrm{C}$, continuously illuminated with $190 \mu \mathrm{mol}$ photons $\mathrm{m}^{-2} \mathrm{~s}^{-1}$ and aerated with a supplement of $1 \% \mathrm{CO}_{2}$. Cells were starved of nitrogen by washing and resuspending them in modified BG11 media, in which $\mathrm{NaNO}_{3}$ was omitted and ammonium ferric citrate was replaced with ferric citrate (Khozin-Goldberg et al., 2002).

\subsection{Isolation of LBs}

All centrifugation steps were performed at $4{ }^{\circ} \mathrm{C}$ using an Eppendorf $5810 \mathrm{R}$ centrifuge for $50 \mathrm{~mL}$ tubes, an Optima LE - 80K ultracentrifuge equipped with an SW40 rotor for $12 \mathrm{~mL}$ thin wall polypropylene tubes and an Optima TLX ultracentrifuge equipped with a TLS55 rotor for $2.5 \mathrm{~mL}$ thin wall polypropylene tubes (all obtained from Beckman Coulter GmbH, Krefeld, Germany).

Buffers used were ice cold and samples were kept on ice between centrifugation steps as well as during resuspension.

An L. incisa culture in the stationary growth phase was starved of nitrogen for 3 days to promote TAG formation, which was verified by Nile Red staining (see section 2.7.1). $50 \mathrm{~mL}$ of culture were centrifuged at $2500 \mathrm{x} g$ for $10 \mathrm{~min}$ to sediment the cells, washed once with distilled water and ground in liquid nitrogen. LBs were then isolated according to (Sturm et al., 1985) with some modifications. The homogenized material was thawed in $60 \mathrm{~mL}$ centrifugation buffer containing $0.6 \mathrm{M}$ sucrose and a $200 \mu \mathrm{L}$ sample of the total extract was transferred to a Protein LoBind $2 \mathrm{~mL}$ reaction tube for later protein isolation. 
The remaining cell extract was transferred to $12 \mathrm{~mL}$ ultracentrifugation tubes and cell debris was sedimented by centrifugation for $10 \mathrm{~min}$ at $10,000 \mathrm{x}$. LBs floating on top along with the entire supernatant were transferred to 2 new ultracentrifugation tubes, each carefully overlain with $10 \mathrm{~mL}$ centrifugation buffer containing $0.4 \mathrm{M}$ sucrose and centrifugation was repeated in order to separate LBs from other cell components. LBs floating on top were collected using a spatula and transferred to a $30 \mathrm{~mL}$ Potter-Elvehjem tissue grinder for resuspension in $10 \mathrm{~mL}$ centrifugation buffer with 0.6 M sucrose using a PTFE pestle. The suspension was once again transferred to an ultracentrifugation tube, overlain with $8 \mathrm{~mL}$ centrifugation buffer with $0.4 \mathrm{M}$ sucrose and centrifuged at $10,000 \mathrm{x}$. The LB fraction was washed this way 2 more times. In the final washing step, LBs were resuspended in $1.5 \mathrm{~mL}$ centrifugation buffer with $0.6 \mathrm{M}$ sucrose using a $5 \mathrm{~mL}$ Potter-Elvehjem tissue grinder, transferred to a $2.5 \mathrm{~mL}$ ultracentrifugation tube and overlain with centrifugation buffer with $0.4 \mathrm{M}$ sucrose before centrifugation at $100,000 \mathrm{xg}$ for $60 \mathrm{~min}$.

$50 \mathrm{~mL}$ of the supernatant from the previous separation step, containing membranes and soluble proteins, were transferred to $412 \mathrm{~mL}$ ultracentrifugation tubes and membranes were sedimented by centrifugation at $105,000 \mathrm{x} g$ for $90 \mathrm{~min}$. A $200 \mu \mathrm{L}$ sample of the supernatant containing soluble proteins was transferred to a Protein LoBind $2 \mathrm{~mL}$ reaction tube and the sedimented membranes were combined in another one.

\subsection{Identification of LB proteins}

\subsubsection{Protein isolation}

Proteins were isolated from the LB fraction as well as the 3 control samples obtained as described in section 2.2 according to (Feussner and Kindl, 1992; Valledor and Weckwerth, 2014) with some modifications. $1.5 \mathrm{~mL}$ ice cold $90 \%$ ethanol were added to each sample and kept at $-80{ }^{\circ} \mathrm{C}$ for $2 \mathrm{~h}$ in order to precipitate proteins, followed by centrifugation at $20,000 \mathrm{xg}$ and $4{ }^{\circ} \mathrm{C}$ for $15 \mathrm{~min}$. After removal of the supernatant the pellets were washed 3 times with ice cold $80 \%$ ethanol and air dried. Proteins were solubilized by adding $100 \mu \mathrm{L}$ denaturing protein solubilization buffer (Laemmli, 1970; Zienkiewicz et al., 2014) and incubating at $37^{\circ} \mathrm{C}$ for $2 \mathrm{~h}$.

\subsubsection{Protein identification}

Proteins in each sample were analyzed 3 times. For each triplicate, $20 \mu \mathrm{L}$ of the sample were loaded on a $10 \%$ sodium dodecyl sulfate polyacrylamide gel (Davis, 1964; Kellenberger, 1968; 
Laemmli, 1970; Ornstein, 1964) and a current of 30 Milliamperes was applied until the dye in the solubilization buffer had migrated approximately $1 \mathrm{~cm}$ into the running gel. The gel was stained for proteins using Coomassie Brilliant Blue G-250 (Meyer and Lamberts, 1965; Neuhoff et al., 1988) and destained overnight in water before the entire band was excised and subjected to an in-gel tryptic digest as previously described (Shevchenko et al., 2007). Briefly, gel pieces were transferred to Protein LoBind tubes and completely destained by incubating at $37^{\circ} \mathrm{C}$ for $30 \mathrm{~min}$ in $50 \%(\mathrm{v} / \mathrm{v})$ acetonitrile containing $200 \mathrm{mM}$ ammonium bicarbonate. Water was removed from the gel by incubation in acetonitrile at room temperature for $10 \mathrm{~min}$ followed by air drying. Sulfide bonds were then reduced with $10 \mathrm{mM}$ dithiotreitol (DTT) at $60{ }^{\circ} \mathrm{C}$ for $15 \mathrm{~min}$ and cysteine residues were blocked by incubation for another 15 min with iodoacetamide at room temperature in the dark. Gel pieces were then washed with $5 \%$ acetonitrile and $50 \mathrm{mM}$ ammonium bicarbonate and equilibrated 2 more times for $15 \mathrm{~min}$ with the same solution before removing the buffer with acetonitrile. Following removal of acetonitrile, samples were incubated over night at $37^{\circ} \mathrm{C}$ with the following amounts of trypsin in $5 \%$ acetonitrile and $50 \mathrm{mM}$ ammonium bicarbonate: $1.2 \mu \mathrm{g}$ (total extract), $0.9 \mu \mathrm{g}$ (each, soluble protein and membrane fraction) and $0.2 \mu \mathrm{g}$ (LB fraction). Supernatants were transferred to new Protein LoBind tubes. Tryptic peptides were extracted from the gel pieces with $1 \%(\mathrm{v} / \mathrm{v})$ formic acid in $60 \%$ acetonitrile at room temperature for $15 \mathrm{~min}$ and for each sample the supernatant was combined with the one from the previous step. Remaining peptides were removed from the gel pieces by incubating them in acetonitrile for another $15 \mathrm{~min}$ at room temperature and combining the supernatant with the previous ones.

The peptides were identified by Dr. Oliver Valerius, University of Göttingen, using liquid chromatography followed by tandem mass spectrometry. All equipment and software used were obtained from Thermo Fisher Scientific, Waltham, USA, unless otherwise stated. An Acclaim PepMap 100 pre-column was employed to wash the peptides (C18 packing material, dimensions: $0.1 \times 2 \mathrm{~cm}, 3 \mu \mathrm{m}$ particle size, $100 \AA$ pore size) with $98 \%$ water, $2 \%$ acetonitrile, $0.07 \%$ trifluoroacetic acid at $25 \mu \mathrm{L} / \mathrm{min}$ for $6 \mathrm{~min}$. Analytical separation of peptides was achieved by reverse phase chromatography using an Acclaim PepMap Rapid Separation Liquid Chromatography column (C18 packing material, dimensions: $0.075 \times 15 \mathrm{~cm}, 3 \mu \mathrm{m}$ particle size, 100 A pore size) with a solvent gradient from $98 \%$ solvent A (99.9\% water, $0.1 \%$ formic acid) and $2 \%$ solvent B (80\% acetonitrile, $19.9 \%$ water, $0.1 \%$ formic acid) to $40 \%$ solvent $B$ within $40 \mathrm{~min}$ at a flow rate of $0.3 \mu \mathrm{L} / \mathrm{min}$. Online ionization took place in a Nanospray Flex Ion 
Source at $2.4 \mathrm{kV}$. Mass over charge ratios of ionized peptides were determined using an Orbitrap Fourier Transform-analyzer (mass over charge range: 300-1850, resolution: 30000) with parallel collision-induced dissociation fragmentation in the Linear Trap Quadrupole Velos Pro ion trap. XCalibur 2.2 software was used for method programming and data acquisition. Identified peptides were searched against a database of predicted proteins generated by the GIAVAP consortium (see beginning of section 0 ) with mass tolerances of 10 parts per million for precursor ions and 0.6 Daltons for fragment ions. Proteome Discoverer software was employed to calculate the false discovery rate based on the number of hits to a decoy database consisting of reversed amino acid sequences of predicted proteins and to filter for a false discovery rate of no more than $1 \%$.

\subsubsection{Enrichment analysis of identified proteins}

Only proteins that were identified in all 3 technical replicates of the LB sample were considered from this point on. For each of these, enrichment compared to the total extract, the membrane fraction and the soluble fraction was determined by calculating its relative abundance in each of the samples based on the number of spectral counts. This requires normalization for protein size, as proteins with a longer amino acid sequence are likely to give rise to more tryptic peptides and thus more MS/MS spectra. The number of spectral counts $(\mathrm{SpC})$ for each protein $k$ was thus divided by the length of its amino acid sequence $(L)$, resulting in the Protein Abundance Factor (PAF) (Powell et al., 2004). As further normalization is necessary to allow comparison of PAFs between different samples, the PAF for each protein in a sample was divided by the sum of the PAFs for all $N$ proteins detected in this sample, yielding the Normalized Spectral Abundance Factor (NSAF) as described previously (Zybailov et al., 2006) (see equation 1).

1

$$
(N S A F)_{k}=\frac{\left(\frac{S p C}{L}\right)_{k}}{\sum_{i=1}^{N}\left(\frac{S p C}{L}\right)_{i}}
$$

NSAF scores were averaged over the 3 technical replicates and for each protein detected in the LB fraction the mean value was divided by its average NSAF score in each of the 3 control samples. The proteins that were highly abundant and strongly enriched in the LB fraction compared to at least one of the controls were selected for further analysis. 


\subsubsection{In silico analyses of putative LB proteins}

The list of identified proteins obtained as described in 2.3.3 was filtered by applying a hierarchy of criteria in order to determine the most promising candidate proteins for further analysis.

Firstly, 2 different algorithms were used to predict the subcellular localization of each protein. TargetP searches for $\mathrm{N}$-terminal presequences in the dataset to predict subcellular localization of the protein and has been tested with datasets from human and A. thaliana samples (Emanuelsson et al., 2000). PredAlgo was specifically developed for datasets from green algae and the algorithm was trained using known transit peptide sequences from the chlorophyte C. reinhardtii (Tardif et al., 2012). Proteins which were predicted by both algorithms to be localized in the mitochondria were not considered any further, whereas those predicted to enter the chloroplast or the secretory pathway as well as those without consensus between both methods were kept as possible candidates.

The remaining amino acid sequences were submitted as queries to the protein Basic Local Alignment Search Tool (pBLAST, Altschul et al., 1990) provided by the National Center for Biotechnology Information (NCBI), Bethesda, USA. Sequences were first searched against a non-redundant database of known proteins from $A$. thalian a and, where no homologs with at least $30 \%$ identity could be found, the search was repeated against a $C$. reinhardtii database. For sequences that showed no or only very low similarity with any entries in these databases, the searches were repeated against all amino acid sequences deposited in the NCBI database.

Amino acid sequences were also searched for conserved domains using the Protein families (PFAM, Finn et al., 2014) database in order to obtain information on possible functions of candidate proteins for which no homolog could be found.

\subsection{Gene expression analysis}

Gene expression was analyzed in L. incis a for several genes of interest. The response of each transcript level to conditions favoring either TAG accumulation or degradation was investigated and the physiological state of the alga was monitored.

\subsubsection{L. incisa growth under conditions of nitrogen starvation and recovery}

A preculture of $L$. incisa was cultivated as described in section 2.1 and diluted with fresh media 3 times during the 2 weeks preceding the experiment to ensure robust growth of the culture. Nitrogen starvation was applied by sedimenting the preculture, washing the cells twice with 
modified BG11 lacking nitrate and ammonium (BG11 -N) and then resuspending them in BG11 -N. The culture was split into 3 main cultures of $300 \mathrm{~mL}$ each and cultivated under the same temperature and light conditions as described before. Following $7 \mathrm{~d}$ of nitrogen depletion, the 3 cultures were sedimented separately and resuspended in full BG11 media for another $3 \mathrm{~d}$ of growth in nitrogen replete conditions.

During the course of the experiment, samples for pigment, lipid and RNA extraction were taken just before as well as 1, 3 and $7 \mathrm{~d}$ following the onset of nitrogen starvation and again 1 and $3 \mathrm{~d}$ after nitrogen resupply. Additional samples for expression analysis were taken $6 \mathrm{~h}$ after each change of media to account for rapid changes in messenger RNA (mRNA) levels.

All samples were frozen in liquid nitrogen and freeze-dried. Pigments were extracted in dimethyl sulfoxide (DMSO) and chlorophyll as well as carotenoid concentrations were determined as described previously (Wellburn, 1994). Briefly, $1 \mathrm{~mL}$ DMSO was added to the sample, which was then heated to $70{ }^{\circ} \mathrm{C}$ for $5 \mathrm{~min}$. Samples were cooled to room temperature, cell debris was sedimented by centrifugation at $3000 \mathrm{x} g$ for $2 \mathrm{~min}$ and the supernatant was transferred to a microcuvette (Sarstedt AG \& Co, Nümbrecht, Germany). Light absorption (A) of the extract was measured at 480, 649 and $665 \mathrm{~nm}$ using an Ultrospec 1100pro spectrophotometer (GE Health Care Life Sciences Ltd., Buckinghamshire, England), subtracting absorption by pure DMSO. Chlorophyll a (Chl a) and b (Chl b) as well as total carotenoid (Car) concentrations were calculated using equations 2 - 4 according to (Wellburn, 1994).

2

$$
[\text { Chl a }]=12.19 \times A_{665}-3.45 \times A_{649}
$$

3

$$
\left[\text { Chl b] }=21.99 \times A_{649}-5.32 \times A_{665}\right.
$$

4

$$
[\text { Car }]=\frac{1000 \times A_{480}-2.14 \times[\text { Chl a }]-70.16 \times[\text { Chl b }]}{221}
$$

The ratio of total carotenoids and chlorophyll was thus calculated using equation 5.

5

$$
\frac{[\mathrm{Car}]}{[\mathrm{Chl}]}=\frac{1000 \times A_{480}-1535.44 \times A_{649}+347.16 \times A_{665}}{1518.27 \times A_{665}+4097.34 \times A_{649}}
$$


Accumulation and degradation of TAG in the course of the experiment were analyzed by quantifying fatty acids in total lipid extracts and in the TAG fraction as described in section 2.9.2.

\subsubsection{Quantitative real-time PCR (qRT-PCR)}

RNA was isolated from samples obtained during the growth experiment and used as a template for complementary DNA (cDNA) synthesis as described in sections 2.5.1 and 2.5.3. The samples were checked for contamination by genomic DNA (gDNA) with RedTaq PCR (see sections 2.5.4 and 2.5.5) using primers specific for actin (gene accession g10221), which gives rise to an amplicon of 1134 base pairs (bp) when cDNA is amplified, but 1409 bp if gDNA serves as a template.

To design primers for the detection of each transcript of interest, the coding nucleotide sequence was first entered into the Primer3Prefold interface (Markham and Zuker, 2008) so as to exclude parts of the sequence that are likely to form secondary structures and thus interfere with amplification. The modified sequence was then entered into the Primer3Plus interface (Untergasser et al., 2012) and oligonucleotides suitable for qRT-PCR were automatically selected considering an amplicon length of 70-150 bp, primer length between 18 and $23 \mathrm{bp}$ and melting temperatures of $58-62{ }^{\circ} \mathrm{C}$ with a difference of less than $3{ }^{\circ} \mathrm{C}$ within the pair. For oligonucleotide sequences see section 3.6.

The gene encoding ribosomal protein S21 was chosen as a reference gene. Normalization to this gene is required to account for changes caused by uneven efficiency of cDNA synthesis as well as alterations in overall transcription due to growth arrest. The gene was chosen based on the observation that its transcript abundance was not significantly changed after 12 or $72 \mathrm{~h}$ of nitrogen starvation according to an RNA sequencing experiment in which 2 biological replicates were measured 4 times each (GIAVAP consortium, unpublished data, see beginning of section 0$)$.

Quantitative real-time measurements were performed using an iQ5 qPCR cycler (BioRad Laboratories GmbH, München, Germany) and the Takyon No Rox SYBR Core Kit blue dTTP (Eurogentec Biologics Division, Seraing, Belgium) according to the manufacturers' instructions. 


\subsection{Isolation and modification of nucleic acids}

Unless otherwise stated, all methods were carried out according to (Green and Sambrook, 2012) and all enzymes used were obtained from Thermo Fisher Scientific, Waltham, USA.

\subsubsection{Isolation of RNA from L. incisa}

Algal material was lyophilized and ground in liquid nitrogen in a $2 \mathrm{~mL}$ reaction tube to ensure effective cell disruption. Total ribonucleic acid (RNA) was then isolated using Trizol extraction (Chomcyzynski and Sacchi, 1987). Briefly, $1 \mathrm{~mL}$ Trizol was added before the material was thawed while thoroughly mixing and incubated at room temperature for $5 \mathrm{~min}$. Cell debris was sedimented by centrifugation at $20,000 \mathrm{x} g$ and $4{ }^{\circ} \mathrm{C}$ for $10 \mathrm{~min}$. The supernatant was transferred to a new $1.5 \mathrm{~mL}$ reaction tube and $200 \mu \mathrm{L}$ chloroform were added. Tubes were shaken vigorously and incubated at room temperature for 2 minutes before centrifugation at 20,000 $\mathrm{g} g$ and $4{ }^{\circ} \mathrm{C}$ for $15 \mathrm{~min}$. The aqueous upper phase containing RNA was once again transferred to a new reaction tube and $1 / 2$ volume isopropanol as well as $1 / 2$ volume high salt precipitation buffer were added to precipitate RNA. The tubes were gently inverted, incubated at room temperature for $10 \mathrm{~min}$ and centrifuged at $20,000 \mathrm{x}$ and $4{ }^{\circ} \mathrm{C}$ for $10 \mathrm{~min}$. The supernatant was removed, the pellet was washed twice with $900 \mu \mathrm{L} 75 \%$ ethanol and dried at room temperature. RNA was dissolved in $20 \mu \mathrm{L}$ RNase-free water.

\subsubsection{Isolation of RNA from Arabidopsis thaliana seeds}

RNA was isolated from $\operatorname{dry} A$. thaliana seeds as described previously (Oñate-Sánchez and Vicente-Carbajosa, 2008). Briefly, $20 \mathrm{mg}$ seeds were collected in a $1.5 \mathrm{~mL}$ reaction tube, cooled in liquid nitrogen and ground with a precooled pestle. $550 \mu \mathrm{L}$ extraction buffer and $550 \mu \mathrm{L}$ chloroform were quickly added, the samples were mixed thoroughly and centrifuged at $4{ }^{\circ} \mathrm{C}$ for $3 \mathrm{~min}$. The supernatant was transferred to a new tube, $500 \mu \mathrm{L}$ phenol saturated with $0.1 \mathrm{M}$ citrate was added followed by vigorous mixing and $200 \mu \mathrm{L}$ chloroform was added before sedimenting again at $4{ }^{\circ} \mathrm{C}$ for $3 \mathrm{~min}$. The supernatant was once again transferred to a new tube, $1 / 3$ volume $8 \mathrm{M} \mathrm{LiCl}$ was added to precipitate $\mathrm{RNA}$ at $-20^{\circ} \mathrm{C}$ for $1 \mathrm{~h}$.

Precipitated nucleic acids were sedimented by centrifugation at $18,000 \mathrm{x} g$ and $4{ }^{\circ} \mathrm{C}$ for $30 \mathrm{~min}$. The pellet was then dissolved in $26 \mu \mathrm{L}$ RNase-free water and residual genomic DNA was removed by adding $1 \mu \mathrm{L}$ DNaseI along with $3 \mu \mathrm{L}$ DNase buffer (Thermo Fisher Scientific, Waltham, USA) and incubating at $37^{\circ} \mathrm{C}$ for $30 \mathrm{~min}$. Subsequently carbohydrates were precipitated by adding $470 \mu \mathrm{L}$ RNase-free water, $7 \mu \mathrm{L} 3 \mathrm{M}$ sodium acetate (pH 5.2) and $250 \mu \mathrm{L}$ 
ethanol and incubating at $4{ }^{\circ} \mathrm{C}$ for $10 \mathrm{~min}$. The supernatant was transferred to a new tube and RNA was precipitated by adding $43 \mu \mathrm{L}$ sodium acetate ( $\mathrm{pH}$ 5.2) and $750 \mu \mathrm{L}$ ethanol, mixing and incubating at $-20{ }^{\circ} \mathrm{C}$ overnight. RNA was then sedimented by centrifugation for $20 \mathrm{~min}$ at $18,000 \times g$ and $4{ }^{\circ} \mathrm{C}$, the pellet was washed with $70 \%$ ethanol and air-dried before resuspension in $20 \mu \mathrm{L} \mathrm{RNase-free} \mathrm{water.}$

\subsection{3 cDNA synthesis}

RNA concentrations were determined by measuring light absorption at $260 \mathrm{~nm}$ wavelength using a NanoDrop 2000 spectrophotometer (Thermo Fisher Scientific, Waltham, USA). Residual DNA was removed by digesting with DNaseI according to the manufacturer's instructions. The entire product of this reaction was then used for cDNA synthesis from messenger RNAs using RevertAid H Minus reverse transcriptase and oligodesoxythymine primers according to the manufacturer's instructions.

\subsubsection{Polymerase chain reaction (PCR)}

DNA sequences were amplified by Polymerase Chain Reaction (PCR) according to (Mullis, 1987). Varying DNA dependent DNA polymerases were used to amplify sequences of interest for different purposes. Phusion Polymerase (New England Biolabs, Ipswich, USA) was used to amplify sequences for further cloning, whereas GoTaq (Promega Corporation, Madison, USA) and RedTaq (Sigma-Aldrich Co., St. Louis, USA) were used to verify the presence of specific DNA sequences. Colony PCR, which serves to verify positive bacterial clones after transformation with a plasmid, was performed by picking a small amount of cells from an agar plate using a pipette tip and resuspending them in the reaction mixture. Reactions were set up according to the manufacturers' instructions and Mastercycler Personal thermocyclers (Eppendorf AG, Hamburg, Germany) were used to ensure appropriate thermal conditions for cycles of DNA denaturing, oligonucleotide annealing and secondary strand elongation. Oligonucleotides used in PCRs for a range of purposes are listed in section 3.6.

\subsubsection{Agarose gel electrophoresis}

Products of PCR and restriction digests were separated in gels consisting of $1 \%(\mathrm{w} / \mathrm{v})$ agarose in Tris Acetic acid EDTA (TAE) buffer based on the methods described previously (Aaij and Borst, 1972; Thorne, 1967). For this purpose, products of Phusion PCR and restriction digests were supplemented with $1 / 5$ volume 6 x DNA loading dye. This was not necessary for GoTaq and RedTaq PCR products, as the respective reaction buffers already contain a loading dye. 
Agarose gels were submerged in TAE buffer, samples were loaded along with a 1 kilobasepair GeneRuler DNA ladder (Thermo Fisher Scientific, Waltham, USA) and a voltage of $10 \mathrm{~V} / \mathrm{cm}$ was applied for $20 \mathrm{~min}$. Gels were then incubated in $2 \mu \mathrm{g} / \mathrm{mL}$ ethidium bromide in TAE buffer for $10 \mathrm{~min}$ before DNA bands were visualized using ultraviolet irradiation and an IDA or DIANA gel documentation system (Raytest Isotopenmessgeräte $\mathrm{GmbH}$, Straubenhardt, Germany).

\subsubsection{Subcloning}

PCR products of the expected length were excised from agarose gels and extracted using the NucleoSpin Gel and PCR Clean-up kit (Macherey-Nagel, Düren, Germany). Products synthesized by Phusion polymerase have blunt ends and could thus be directly ligated into the pJET1.2/blunt subcloning vector using the CloneJET PCR cloning kit (Thermo Fisher Scientific, Waltham, USA) according to the manufacturer's instructions with minor modifications. Reactions were set up in a total volume of $10 \mu \mathrm{L}$ and ligation was carried out for $30 \mathrm{~min}$ at room temperature. The DNA fragment to be inserted and the vector were used in a molar ratio of 3:1 as calculated using the online tool provided by the Directed Evolution group of the University of Düsseldorf, Germany.

The entire ligation mixture was used to transform $100 \mu \mathrm{L}$ chemically competent Escherichia coli (E. coli) XL1-Blue cells (Agilent Technologies, Santa Clara, USA) by heat shock (for generation of competent cells see section 2.5.9). Briefly, the cells were incubated on ice with the ligation product for $30 \mathrm{~min}$ followed by a heat shock of $42{ }^{\circ} \mathrm{C}$ for $45 \mathrm{~s}$. The mixture was then placed back on ice for another 2 min before $900 \mu \mathrm{L}$ Lysogeny broth (LB) was added. The cells were shaken at $37^{\circ} \mathrm{C}$ for $1 \mathrm{~h}$ and then plated on LB agar plates containing $100 \mu \mathrm{g} / \mathrm{mL}$ carbenicillin for selection of positive clones. Plates were incubated at $37^{\circ} \mathrm{C}$ overnight and positive clones were verified by colony PCR followed by agarose gel electrophoresis (see sections 2.5.4 and 2.5.5).

Positive bacterial clones were used to inoculate $4 \mathrm{~mL} \mathrm{LB}$ containing $100 \mu \mathrm{g} / \mathrm{mL}$ carbenicillin and shaken over night at $37^{\circ} \mathrm{C}$. Plasmids were isolated using the GenElute HP Plasmid Miniprep kit (Sigma-Aldrich Co., St. Louis, USA) according to the manufacturer's instructions. They were then subjected to control restriction digests (see section 2.5.7) followed by agarose gel electrophoresis to compare the lengths of the resulting DNA fragments with the expected restriction fragments. Verified plasmids were submitted to GATC Biotech AG, Konstanz, 
Germany for sequencing. Sequencing results were compared with the expected sequence using Geneious R8 software (Biomatters Ltd., Auckland, New Zealand).

\subsubsection{Restriction cloning}

Restriction cloning was used to transfer DNA fragments of interest from the subcloning vector to other vectors for expression or Gateway cloning. Reactions were set up according to the manufacturer's instructions either in a $50 \mu \mathrm{L}$ volume overnight for preparative digests or in a $10 \mu \mathrm{L}$ volume for $1 \mathrm{~h}$ for control digests.

For restriction cloning, the destination vector and the DNA fragment to be inserted into it were digested with enzymes resulting in the same or compatible overhangs. The products of preparative digests were separated by agarose gel electrophoresis and the band of expected size was excised. DNA was extracted, ligated to the destination vector and used for transformation of competent E. coli XL1-Blue cells as previously described (see section 2.5.6).

A higher concentration of plasmids was required for transformation of N. tabacum pollen grains, thus for each plasmid $50 \mathrm{~mL} \mathrm{LB}$ media with $100 \mu \mathrm{g} / \mathrm{mL}$ carbenicillin was inoculated with a positive E. coli XL1-Blue clone and shaken at $37^{\circ} \mathrm{C}$ overnight. High amounts of plasmid were then isolated using the GenElute HP Plasmid Midiprep kit (Sigma-Aldrich Co., St. Louis, USA) according to the manufacturer's instructions.

\subsubsection{Gateway cloning}

The Gateway cloning system (Thermo Fisher Scientific, Waltham, USA) was used to introduce genes of interest into the relatively large vectors required for stable transformation of A. thaliana. For vectors used see section 9.3.

Initially, DNA fragments of interest were inserted into Entry vectors as described in section 2.5.7. The Entry vector pUC18-Entry was used for subsequent transfer of the DNA fragment into the expression vector pCambia 23.1, while pEntry-E was used for transfer into pCambia 43.0 by Gateway cloning according to the manufacturer's instructions. Unmodified pEntry-E was used in Gateway reactions to generate pCambia plasmids lacking a coding sequence in the open reading frame. These plasmids were used to transform plants that served as empty vector controls.

Briefly, 10 fmol Entry vector containing the gene of interest was mixed with 20 femtomol expression vector, $1 \mu \mathrm{L}$ LR Clonase II enzyme mix and Tris EDTA buffer (TE buffer) in a total volume of $10 \mu \mathrm{L}$. The reaction mixture was incubated at $25{ }^{\circ} \mathrm{C}$ overnight and $1 \mu \mathrm{L}$ Proteinase $\mathrm{K}$ 
was added followed by incubation at $37^{\circ} \mathrm{C}$ for $10 \mathrm{~min}$ to stop the reaction. The proteinase was inactivated by incubation at $70{ }^{\circ} \mathrm{C}$ for $10 \mathrm{~min}$ and the entire mixture was used in transformation of $100 \mu \mathrm{L}$ competent E. coli DH5- $\alpha$ cells (New England Biolabs, Ipswich, USA). Transformation of bacteria was carried out as described in section 2.5.6, except for a prolonged incubation of 90 min after heat shock transformation and LB agar plates containing $25 \mu \mathrm{g} / \mathrm{mL}$ kanamycin instead of carbenicillin for selection of positive clones. Following incubation on agar plates overnight, individual colonies were each transferred to a new LB plate containing kanamycin as well as a second plate containing carbenicillin. The following day, only those colonies that had grown on kanamycin but not on carbenicillin, and therefore did not contain the Entry plasmid anymore, were used in colony PCR (see section 2.5.4).

\subsubsection{Generation of chemically competent bacteria}

Bacteria were made competent for the uptake of plasmid DNA by chemical treatment. Overnight cultures of E. coli XL1-Blue or DH5- $\alpha$ were used to inoculate a main culture which was shaken at $37^{\circ} \mathrm{C}$ until an optical density at $600 \mathrm{~nm}\left(\mathrm{OD}_{600}\right)$ of $0.45-0.75$ was reached. Cells were chilled on ice and sedimented at $1000 \mathrm{xg}$ and $4{ }^{\circ} \mathrm{C}$ for $10 \mathrm{~min}$. The supernatant was removed and the cells were resuspended in ice cold TFP buffer followed by an additional $10 \mathrm{~min}$ incubation on ice. This treatment was repeated once before dimethylsulfoxide (DMSO) was added to a final concentration of $7 \%(\mathrm{v} / \mathrm{v})$. Cells were incubated on ice for another $10 \mathrm{~min}$, frozen in liquid nitrogen and stored at $-80^{\circ} \mathrm{C}$.

An $A$. tumefaciens EHA 105 culture was shaken at $28^{\circ} \mathrm{C}$ overnight and used to inoculate a main culture that was cultivated under the same conditions until an $\mathrm{OD}_{600}$ of 0.5 was reached. The cells were sedimented at $4000 \mathrm{x} g$ and $4{ }^{\circ} \mathrm{C}$ for $15 \mathrm{~min}$, the supernatant was removed and the cells were gently resuspended in ice cold $0.15 \mathrm{M} \mathrm{NaCl}$. The cell suspension was centrifuged again, the supernatant was removed and the cells were resuspended in ice cold $75 \mathrm{mM} \mathrm{CaCl}_{2}$. They were then frozen in liquid nitrogen and stored at $-80{ }^{\circ} \mathrm{C}$.

\subsection{Transient gene expression in Nicotiana tabacum pollen tubes}

LB localization of L. incis a proteins was confirmed by transiently expressing the corresponding genes fused to a fluorescent marker in N. tabacum (ecotype SNN) pollen tubes. Using the methods described in sections 2.5.4 - 2.5.7, C-terminal fusions of algal coding sequences were created by removing the stop codon and inserting them into a pUC19 vector containing the 
mVenus cDNA fragment and the pollen specific Lat52 promoter from Solanum lycopersicum (Mähs et al., 2013; Twell et al., 1989). Pollen grains were transformed as described previously (Read et al., 1993). Briefly, $10 \mathrm{mg}$ gold particles with a diameter of $1 \mu \mathrm{m}$ (BioRad Laboratories GmbH, München, Germany) were washed repeatedly with ethanol and finally resuspended in sterile bidistilled water. The suspension was split into 8 aliquots of $50 \mu \mathrm{L}$ that were coated with copies of one plasmid each.

$8 \mu \mathrm{g}$ highly concentrated plasmid obtained as described in 2.5.7 were added to the gold particles to a final volume of no more than $55 \mu \mathrm{L}$ and mixed vigorously. DNA was precipitated and coated onto the gold particles by adding $50 \mu \mathrm{L} 2.5 \mathrm{M} \mathrm{CaCl}_{2}$ and then $20 \mu \mathrm{L} 0.1 \mathrm{M}$ spermidine while mixing thoroughly. The coated particles were washed 3 times with ethanol and resuspended in $60 \mu \mathrm{L}$ ethanol.

For each gene to be expressed, pollen from 6 to $8 N$. tabacum flowers were harvested by collecting stamens in a $15 \mathrm{~mL}$ conical tube (Sarstedt AG \& Co, Nümbrecht, Germany). They were suspended in $2 \mathrm{~mL}$ pollen tube growth media (PT media) and quickly transferred to a cellulose acetate filter (Sartorius AG, Göttingen, Germany) by vacuum filtration, which was then placed on a piece of filter paper (GE Health Care Life Sciences Ltd., Buckinghamshire, England) soaked in PT media to avoid dehydration of the pollen grains. The PDS1000/He Biolistic Particle Delivery System (BioRad Laboratories GmbH, München, Germany) was used for particle bombardment according to the manufacturer's instructions. $20 \mu \mathrm{L}$ gold particle suspension were used for each bombardment and rupture discs that can withstand a pressure of 1350 pounds per square inch were used with a vacuum equivalent to 28 inches of mercury. Pollen grains were scraped off and resuspended in $250 \mu \mathrm{L}$ PT media. $60 \mu \mathrm{L}$ suspension were distributed onto each of 4 glass slides (Carl Roth GmbH \& Co. KG, Karlsruhe, Germany), which were then incubated at room temperature and high humidity for $6 \mathrm{~h}$ before analysis.

\subsection{Microscopy}

\subsubsection{Detection of neutral lipids in L. incisa cells and isolated LBs}

$1 \mathrm{~mL} \mathrm{~L}$. incisa culture that had been starved of nitrogen for $3 \mathrm{~d}$ was stained for neutral lipids according to (Greenspan et al., 1985) by adding $1 \mu \mathrm{L}$ of $1 \mathrm{mg} / \mathrm{mL}$ Nile Red in acetone, mixing carefully and incubating at room temperature for 5 min in the dark.

For isolated LBs (see section 2.2), a $1 \mu \mathrm{L}$ sample was carefully resuspended in $1 \mathrm{~mL}$ water using a Potter-Elvehjem tissue grinder and then stained in the same way. 
Nile Red fluorescence was observed using a BX51 microscope equipped with a U-RFL-T mercury lamp. A U-MWB2 filter was used to allow excitation of the fluorescent dye at 460$490 \mathrm{~nm}$ wavelength and detection of emission above $520 \mathrm{~nm}$. Images were acquired using a ColorView II camera and analySis 3.2 software (all obtained from Olympus Corporation, Tokyo, Japan).

\subsubsection{Localization studies in N. tabacum pollen tubes}

Subcellular localization of transiently expressed proteins in tobacco pollen tubes was analyzed by confocal fluorescence microscopy using a Laser Scanning Microscope 510 (Carl Zeiss Microscopy GmbH, Jena, Germany). For colocalization with Nile Red, the pollen tube suspension on the glass slide was supplemented dropwise with $20 \mu \mathrm{L}$ fixation solution consisting of PT media with $4 \%$ (w/v) formaldehyde and $1 \mu \mathrm{g} / \mathrm{mL}$ Nile Red in acetone. An argon laser and a helium-neon laser were used for excitation of mVenus and Nile Red at 488 and $543 \mathrm{~nm}$, respectively. A HFT 488/543 main dichroic beam splitter directed only the excitation light to the sample and a $505-530 \mathrm{~nm}$ bandpass filter was used for the detection of green fluorescence, while a $585 \mathrm{~nm}$ long pass filter was used to detect fluorescence emitted by Nile Red.

2.7.3 Selection of Arabidopsis thaliana seeds expressing the $m$ Cherry gene Seeds expressing $m$ Cherry as a reporter gene were identified using an M165C stereomicroscope equipped with an EL6000 mercury lamp, a M205FA/M165FC filter set and a DFC3000 G camera (all obtained from Leica Microsystems GmbH, Wetzlar, Germany). 


\subsection{Complementation of Arabidopsis thaliana mutants}

Unless otherwise stated, the methods in this section were adapted from (Weigel and Glazebrook, 2002). For plant lines used, see section 3.6.1.

\subsubsection{Arabidopsis thaliana growth conditions}

For sterile culture seeds were surface sterilized by exposing them to chlorine gas for $3 \mathrm{~h}$. In a desiccator under a fume hood, $50 \mathrm{~mL} 12 \%(\mathrm{w} / \mathrm{v})$ sodium hypochlorite was mixed with $5 \mathrm{~mL}$ $32 \%(\mathrm{v} / \mathrm{v})$ hydrochloric acid. Immediately afterwards, open reaction tubes containing the seeds were placed in the desiccator and the lid was closed. After $3 \mathrm{~h}$, the seeds were removed and placed in a laminar flow hood for several hours to remove residual gas that could inhibit germination. The seeds were subsequently taken up in $0.1 \%$ microagar (Duchefa Biochemie B.V, Haarlem, The Netherlands) and spread on 1/2 Murashige-Skoog (including vitamins) (Murashige and Skoog, 1962) (1/2 MS) agar plates containing $1 \%$ sucrose. Plates were allowed to dry before being sealed with surgical tape (3M Deutschland GmbH, Neuss, Germany) and stratified for $3 \mathrm{~d}$ at $4{ }^{\circ} \mathrm{C}$ in the dark in order to synchronize germination. The plates were then placed under constant illumination at room temperature until seedlings were large enough to be transferred to soil.

Soil (Fruhstorfer Erde Typ T fein, Hawita Gruppe GmbH, Vechta, Germany) was steamed at $80{ }^{\circ} \mathrm{C}$ for $8 \mathrm{~h}$ and then treated with $0.25 \%$ (v/v) Previcur fungicide (Bayer AG, Leverkusen, Germany) before being used in non-sterile $A$. thalian a culture. Seedlings were carefully placed in soil, covered with a plastic hood for several days and transferred to a climate chamber (York Industriekälte $\mathrm{GmbH} \& \mathrm{Co} . \mathrm{KG})$. Growth conditions were set to $22{ }^{\circ} \mathrm{C}, 16 \mathrm{~h}$ light $/ 8 \mathrm{~h}$ dark and $60 \%$ humidity. For plants that were to be transformed, the first inflorescence was cut off in order to induce the growth of further inflorescence meristems. Other plants were directly transferred to the greenhouse as soon as they started flowering.

\subsubsection{Transformation of Agrobacteria}

Agrobacterium tumefaciens EHA 105 cells were transformed by heat shock according to (Höfgen and Willmitzer, 1988) with minor modifications. Briefly, $200 \mu \mathrm{L}$ competent cells were thawed on ice and $3 \mu \mathrm{g}$ plasmid was added. The mixture was incubated on ice for $30 \mathrm{~min}$, frozen at $-80{ }^{\circ} \mathrm{C}$ for $3 \mathrm{~min}$ and incubated at $37^{\circ} \mathrm{C}$ for $5 \mathrm{~min}$ before adding $800 \mu \mathrm{L} \mathrm{LB}$ media. The cells were incubated at $28{ }^{\circ} \mathrm{C}$ for $4 \mathrm{~h}$ and then spread on LB agar plates containing $50 \mu \mathrm{g} / \mathrm{mL}$ rifampicin (Duchefa Biochemie B.V, Haarlem, The Netherlands) and $25 \mu \mathrm{g} / \mathrm{mL}$ kanamycin for 
selection of positive clones. Plates were sealed with Parafilm (Bemis Company Inc., Neenah, USA) and incubated at $28{ }^{\circ} \mathrm{C}$ for $2 \mathrm{~d}$. Individual colonies were transferred to a new plate, incubated again for $1 \mathrm{~d}$ and stored at $4{ }^{\circ} \mathrm{C}$.

\subsubsection{Agrobacterium-mediated transformation of Arabidopsis thaliana}

Stable transformants of $A$. thaliana were generated by flower dip with $A$. tumefaciens. A $500 \mathrm{~mL}$ culture of transgenic Agrobacteria in LB media containing antibiotics as described in section 2.8.2 was shaken for $2 \mathrm{~d}$ at $28{ }^{\circ} \mathrm{C}$ until an $\mathrm{OD}_{600}$ of at least 0.8 was reached. Cells were sedimented by centrifugation at $7000 \mathrm{x} g$ and $4{ }^{\circ} \mathrm{C}$ for $20 \mathrm{~min}$ using an Avanti J25 centrifuge with a JA-10 rotor (Beckman Coulter GmbH, Krefeld, Germany) and resuspended in $300 \mathrm{~mL}$ cold 5\% sucrose solution. $70 \mu \mathrm{L}$ Silwet L-77 silicone surfactant (Momentive Performance Materials Inc., Waterford, USA) was added just before dipping the flowers in the bacterial suspension and gently agitating them for several seconds. Plants were then covered with a plastic hood overnight and transferred to the greenhouse. Transformation was repeated after 1 week to increase the number of transgenic seeds.

\subsubsection{Selection of transgenic plants}

Seeds expressing $m$ Cherry as a reporter gene were identified using a fluorescence stereomicroscope as described in section 2.7.3.

The $s d p 1 / s d p 1-L$ line used in this study (see section 3.6.1) was originally generated by crossing the single knockout lines SALK_076697 (Eastmond, 2006) and European Arabidopsis Stock Center N873426 (Kelly et al., 2011) and is thus resistant to both glufosinate and kanamycin. Seeds from plants that had been transformed with a plasmid lacking the $m$ Cherry reporter gene were therefore screened for functional complementation of the mutant etiolated seedling phenotype by the introduced algal gene (see section 2.8.5). Seedlings with elongated hypocotyls as compared to mutant seeds carrying only the empty vector were carefully transferred to $1 / 2 \mathrm{MS}$ agar plates containing $1 \%(\mathrm{w} / \mathrm{v})$ sucrose and placed under constant illumination. Plants were then grown as described in section 2.8.1 and transgene insertion was confirmed by PCR following genomic DNA extraction according to (Doyle, 1987). Briefly, plant material was placed in a $1.5 \mathrm{~mL}$ reaction tube and ground in liquid nitrogen using a precooled plastic pestle. $250 \mu \mathrm{L}$ Cetyltrimethylammoniumbromide (CTAB) extraction buffer was added to the frozen material and incubated at $65^{\circ} \mathrm{C}$ for $15 \mathrm{~min}$. An equal volume of chloroform:isoamyl alcohol $(24: 1 \mathrm{v} / \mathrm{v})$ was added and after thorough mixing phase separation was ensured by centrifugation 
for $3 \mathrm{~min}$ at $7500 \mathrm{x} g$ and room temperature. $200 \mu \mathrm{L}$ of the aqueous phase was then transferred to a new tube, $200 \mu \mathrm{L}$ isopropanol was added and the tubes were inverted several times. Following incubation at room temperature for $2 \mathrm{~min}$, the extract was centrifuged at room temperature and 20,000 $\mathrm{x} g$ for $10 \mathrm{~min}$ to sediment DNA. The pellet was washed with $100 \mu \mathrm{L}$ ethanol and then air-dried before redissolving in $70 \mu \mathrm{L}$ water. Transgenes were detected by RedTaq PCR as described in section 2.5.4.

\subsubsection{Hypocotyl growth assay}

Functional complementation of postgerminative growth conferred by L. incisa genes heterologously expressed in the A. thaliana $s d p 1 / s d p 1-L$ mutant was tested by observing hypocotyl length of etiolated T2 seedlings as described previously (Kelly et al., 2011). Initially, seeds from each independent line were selected for similar size $(250-300 \mu \mathrm{m})$ by sifting through wash sieves (Advantech Manufacturing Inc., New Berlin, USA). Seeds were then surface sterilized and placed on $1 / 2$ MS agar plates with and without $1 \%$ sucrose as described in section 2.8.1 before transfer to $4{ }^{\circ} \mathrm{C}$ to synchronize germination by stratification for $2 \mathrm{~d}$. As the transgene is still segregating in this generation, red fluorescence of individual seeds was documented at this point for lines carrying the $m$ Cherry reporter gene (see section 2.8.4). The plates were then exposed to light for another $30 \mathrm{~min}$ to induce germination and afterwards kept upright in the dark at room temperature for $5 \mathrm{~d}$. Plates were documented using a CanonScan 8000F scanner (Canon Incorporated, Tokyo, Japan) and the length of seedlings originating from fluorescing seeds was measured using ImageJ software (Rasband). For plant lines not carrying the fluorescent reporter, seedlings from agar plates without sucrose were individually transferred to plates with $1 \%$ sucrose and cultivated for an additional week under continuous illumination. In this way, sufficient material was obtained to extract genomic DNA for a posteriori identification of transgenic and non-transgenic seedlings (see section 2.8.4). For each line, the hypocotyl length in 4 batches of at least 10 transgenic seedlings was determined.

The fatty acid content of these seedlings was determined by gas chromatography as described in section 2.9.1. For each independent line, 4 samples of at least 10 seedlings were collected on ice and stored at $-20^{\circ} \mathrm{C}$. In order to relate the fatty acid content of etiolated seedlings to that of seeds, 4 batches of 10 seeds were analyzed per line as well. 


\subsubsection{Lipase activity assay}

TAG lipase activity of L. incisa putative lipases was tested as previously described (Eastmond, 2006) by measuring the degradation of radioactively labeled TAG in crude cell extracts of germinated $s d p 1 / s d p 1$ - $L$ seedlings expressing the algal gene. Briefly, $6 \mathrm{mg}$ seeds from each independent line were surface sterilized, taken up in sterile water and germinated on $1 / 2$ MS agar plates with $1 \%$ sucrose as described in section 2.8.1. After $3 \mathrm{~d}$ germination at room temperature, the seedlings were carefully scraped off the agar and collected in $1.5 \mathrm{~mL}$ reaction tubes on ice, ground in $250 \mu \mathrm{L}$ grinding buffer using a precooled plastic pestle and the pestle was washed with an additional $250 \mu \mathrm{L}$ grinding buffer. Cell debris was sedimented by centrifugation at $4{ }^{\circ} \mathrm{C}$ and $100 \mathrm{x} g$ for 1 min before transfer of the supernatant to a new tube on ice.

Total protein concentration was measured according to (Bradford, 1976). $5 \mu \mathrm{L}$ sample were diluted 10-fold in Bradford reagent, incubated at room temperature for $10 \mathrm{~min}$ and absorbance was measured at $595 \mathrm{~nm}$ wavelength. Bovine serum albumin was used as a standard to calculate protein concentration in the sample.

A volume of each sample corresponding to $40 \mu \mathrm{g}$ total protein (or grinding buffer as a control) was diluted to a final volume of $250 \mu \mathrm{L}$ with buffer at $\mathrm{pH}$ 6, 7 or 8 in glass vials. Carboxyl- ${ }^{14} \mathrm{C}$ triolein (PerkinElmer Inc., Waltham, USA) or ${ }^{14} \mathrm{C}$-palmitic acid (GE Health Care Life Sciences Ltd., Buckinghamshire, England) were each diluted 1:10 in ethanol, $2.5 \mu \mathrm{L}$ was added and the reaction was incubated at room temperature for $2 \mathrm{~h}$. The reaction was stopped by adding $200 \mu \mathrm{L}$ hexane and mixing. The entire reaction was then transferred to $1.5 \mathrm{~mL}$ reaction tubes and centrifuged at $18,000 \mathrm{xg}$ for $1 \mathrm{~min}$ to promote phase separation. The hexane phase was transferred to a new tube and the aqueous phase was re-extracted with $500 \mu \mathrm{L}$ hexane. The supernatants were then combined and evaporated under a stream of nitrogen before dissolving the lipids in $50 \mu \mathrm{L}$ hexane.

The entire sample was applied to a TLC plate and a solvent system consisting of chloroform:acetone $(96: 4 \mathrm{v} / \mathrm{v})$ was used as a mobile phase. The plate was air-dried, wrapped in cling foil and placed under a BAS-MP imaging plate (Fujifilm Corporation, Tokyo, Japan). The plate was exposed for 15 min to visualize radioactive radiation emitted by free fatty acids and TAG. It was read using a FLA-3000 fluorescent image analyzer (Fujifilm Corporation, Tokyo, Japan), the intensity of the band corresponding to TAG was measured using ImageJ and related to the sum of signals detected for the sample. 


\subsection{Analysis of fatty acids}

2.9.1 Analysis of fatty acids in Arabidopsis thaliana seeds and seedlings

Fatty acids in A. thaliana material were quantified by gas chromatography (GC) following acidic methanolysis according to (Miquel and Browse, 1992). Water was removed from the samples by drying at $60{ }^{\circ} \mathrm{C}$ overnight (for seeds) or freeze-drying for $1 \mathrm{~d}$ using a Lyovac GT 3 freeze dryer (GEA Lyophil GmbH, Hürth, Germany, for frozen seedlings). Varying amounts of tri-15:0 in toluol were added to the different types of samples as an internal standard: $20 \mu \mathrm{g}$ (for seeds), $15 \mu \mathrm{g}$ (for $s d p 1 / s d p 1-L$ seedlings carrying only the empty vector), $8 \mu \mathrm{g}$ (for seedlings of complemented lines) and $1.5 \mu \mathrm{g}$ (for wildtype seedlings). Acidic methanolysis of fatty acids was achieved by adding $1 \mathrm{~mL}$ fatty acid methyl ester (FAME) solution and incubating at $80{ }^{\circ} \mathrm{C}$ for $1 \mathrm{~h}$. The reaction was stopped by adding $200 \mu \mathrm{L} 5 \mathrm{M} \mathrm{NaCl}$ and FAMEs were extracted with $2 \mathrm{~mL}$ hexane. Phase separation was promoted by centrifugation at $1000 \mathrm{x} \mathrm{g}$ for 2 min, the hexane phase was transferred to a new glass vial and the remainder was extracted a second time with $2 \mathrm{~mL}$ hexane. The combined supernatants were dried under a stream of nitrogen, dissolved in $200 \mu \mathrm{L}$ acetonitrile and transferred to the inlay of a GC vial. Lipids were once again dried under a stream of nitrogen, dissolved in $20 \mu \mathrm{L}$ acetonitrile and quantified by gas chromatography. An Agilent Gas Chromatograph 6890 (Agilent Technologies, Santa Clara, USA) equipped with a capillary DB-23 column ( $30 \mathrm{~m}$ x $0.25 \mathrm{~mm}, 0.25 \mu \mathrm{m}$ coating thickness, J\&W Scientific, Agilent, Waldbronn, Germany) and a flame ionization detector (FID) were used with the following temperature gradient:

$150{ }^{\circ} \mathrm{C}$ for $1 \mathrm{~min}$

$150{ }^{\circ} \mathrm{C}$ to $200{ }^{\circ} \mathrm{C}$ at $4{ }^{\circ} \mathrm{C} / \mathrm{min}(12.5 \mathrm{~min})$

$200{ }^{\circ} \mathrm{C}$ to $250{ }^{\circ} \mathrm{C}$ at $20^{\circ} \mathrm{C} / \mathrm{min}(2.5 \mathrm{~min})$

$250{ }^{\circ} \mathrm{C}$ for $3 \mathrm{~min}$.

Samples were measured in sequence with a standard mixture of FAMEs with a known pattern of retention times (C14-C24, Sigma-Aldrich Co., St. Louis, USA) to allow identification of the fatty acids. For each fatty acid identified, the peak area was integrated using Agilent ChemStation software (Agilent Technologies, Santa Clara, USA). Peak area was normalized to account for differences in molecular weight, related to the amount of fatty acids derived from the internal standard and divided by the number of seeds or seedlings in the sample. In $A$. thaliana seeds, 
20:1 (n-9) is almost exclusively present in TAG (Lemieux et al., 1990) and was therefore used as a marker for TAG levels.

\subsubsection{Analysis of fatty acids in TAG and total extracts of L. incisa}

Lipids from L. incisa material were extracted prior to methanolysis and TAG was separated from other lipid classes by thin layer chromatography (TLC).

Methyl tert-butyl ether (MTBE) extraction was performed according to (Matyash et al., 2008). Briefly, $1 \mathrm{~mL}$ isopropanol at $75^{\circ} \mathrm{C}$ was added to $10 \mathrm{mg}$ lyophilized algal material along with $50 \mu \mathrm{L}$ tri-15:0 (1 mg/mL) and 100 $\mu \mathrm{L}$ di-17:0 phosphatidylcholine $(1 \mathrm{mg} / \mathrm{mL}$, Larodan Fine Chemicals AB, Limhamn, Sweden) as internal standards. The reaction was covered with argon to prevent oxidation and heated to $75^{\circ} \mathrm{C}$ for $15 \mathrm{~min}$. Isopropanol was allowed to evaporate, the reaction was cooled to room temperature and $750 \mu \mathrm{L}$ methanol as well as $2.5 \mathrm{~mL}$ MTBE were added. Lipids were extracted by shaking at $4{ }^{\circ} \mathrm{C}$ for $1 \mathrm{~h}$ before sonifying the samples for $10 \mathrm{~min}$ in a Sonorex RK 510S sonification bath (Bandelin electronic GmbH \& Co. KG, Berlin, Germany). $600 \mu \mathrm{L}$ water was added, samples were mixed vigorously and incubated at room temperature for $10 \mathrm{~min}$ before centrifugation at $3000 \mathrm{xg}$ for $15 \mathrm{~min}$ to promote phase separation. The MTBE-phase was transferred to a new glass vial and the aqueous phase was re-extracted with $700 \mu \mathrm{L}$ methanol/water 3:2.5 (v/v) and $1.3 \mathrm{~mL}$ MTBE. The resulting MTBE phase was combined with the first one, dried under a stream of nitrogen and taken up in $100 \mu \mathrm{L}$ chloroform.

TAG was separated from other lipid classes in the extract by TLC according to (Blank and Snyder, 1975). $40 \mu \mathrm{L}$ lipid extract was applied to a Silica gel 60 plate (Merck KGaA, Darmstadt, Germany) a solvent system consisting of hexane:diethyl ether:acetic acid (80:20:1, v/v/v) was used as the mobile phase. The plate was air-dried, sprayed with $0.2 \%(\mathrm{w} / \mathrm{v})$ 8-anilinonaphthalene-1-sulfonic acid (ANS) and irradiated with UV light to visualize lipids. The TAG fraction was scraped out and fatty acids were derivatized by acidic methanolysis for gas chromatographic analysis as described in section 2.9.1.

In addition to TAG quantification, total lipid extracts obtained by MTBE extraction were directly derivatized using the method described above (section 2.9.1), using the internal 17:0 standard to quantify fatty acids. 


\section{Materials}

\subsection{Equipment}

Table 1: Equipment used in this study. Listed below is the equipment used in this study, along with the supplier.

\begin{tabular}{|l|l|}
\hline & Supplier \\
\hline 400 mL glass columns for algae cultivation & Ochs GmbH, Bovenden, Germany \\
\hline Agilent Gas Chromatograph 6890 & Agilent Technologies, Santa Clara, USA \\
\hline Avanti J25 centrifuge & Beckman Coulter GmbH, Krefeld, Germany \\
\hline BAS-MP imaging plate & Fujifilm Corporation, Tokyo, Japan \\
\hline BX51 microscope & Olympus Corporation, Tokyo, Japan \\
\hline CanonScan 8000F scanner & Canon Incorporated, Tokyo, Japan \\
\hline climate chamber & York Industriekälte GmbH \& Co. KG \\
\hline ColorView II camera & Olympus Corporation, Tokyo, Japan \\
\hline DB-23 column for gas chromatography & J\&W Scientific, Agilent, Waldbronn, Germany \\
\hline DFC3000 G camera & Leica Microsystems GmbH, Wetzlar, Germany \\
\hline EL6000 mercury lamp & Leica Microsystems GmbH, Wetzlar, Germany \\
\hline FLA-3000 fluorescent image analyzer & Fuiifilm Corporation, Tokyo, Japan \\
\hline $\begin{array}{l}\text { IDA and DIANA gel documentation } \\
\text { systems }\end{array}$ & $\begin{array}{l}\text { Raytest Isotopenmessgeräte GmbH, } \\
\text { Straubenhardt, Germany }\end{array}$ \\
\hline iQ5 qPCR cycler & $\begin{array}{l}\text { BioRad Laboratories GmbH, München, } \\
\text { Germany }\end{array}$ \\
\hline JA10 rotor & Beckman Coulter GmbH, Krefeld, Germany \\
\hline Laser Scanning Microscope 510 & Carl Zeiss Microscopy GmbH, Jena, Germany \\
\hline Lyovac GT 3 freeze dryer & GEA Lyophil GmbH, Hürth, Germany \\
\hline M165C stereomicroscope & Leica Microsystems GmbH, Wetzlar, Germany \\
\hline M205FA/M165FC filter set & Leica Microsystems GmbH, Wetzlar, Germany \\
\hline Mastercycler Personal thermocycler & Eppendorf AG, Hamburg, Germany \\
\hline Mini-PROTEAN3 electrophoresis system & $\begin{array}{l}\text { BioRad Laboratories GmbH, München, } \\
\text { Germany }\end{array}$ \\
\hline NanoDrop 2000 spectrophotometer & Thermo Fisher Scientific, Waltham, USA \\
\hline Optima TLX ultracentrifuge & Beckman Coulter GmbH, Krefeld, Germany \\
\hline Optima LE - 80K ultracentrifuge & Beckman Coulter GmbH, Krefeld, Germany \\
\hline $\begin{array}{l}\text { PDS1000/He Biolistic Particle Delivery } \\
\text { System }\end{array}$ & $\begin{array}{l}\text { BioRad Laboratories GmbH, München, } \\
\text { Germany }\end{array}$ \\
\hline $\begin{array}{l}\text { Potter-Elvehjem tissue grinder (5 and } \\
\text { 30mL) and PTFE pestles }\end{array}$ & Wheaton Industries Inc., Millville, USA \\
\hline Sonorex RK 510S sonification bath & $\begin{array}{l}\text { Bandelin electronic GmbH \& Co. KG, Berlin, } \\
\text { Germany }\end{array}$ \\
\hline TLS55 ultracentrifuge rotor & Beckman Coulter GmbH, Krefeld, Germany \\
\hline
\end{tabular}


Table 1, continued

\begin{tabular}{|l|l|}
\hline Ultrospec 1100pro spectrophotometer & $\begin{array}{l}\text { GE Health Care Life Sciences Ltd., } \\
\text { Buckinghamshire, England }\end{array}$ \\
\hline U-MWB2 filter for Nile Red & Olympus Corporation, Tokyo, Japan \\
\hline U-RFL-T mercury lamp & Olympus Corporation, Tokyo, Japan \\
\hline Wash sieves & $\begin{array}{l}\text { Advantech Manufacturing Inc., New Berlin, } \\
\text { USA }\end{array}$ \\
\hline
\end{tabular}

\subsection{Software}

Table 2: Software and web-based services used in this study. Given below is a list of all software and web-based services used in this study, along with the associated reference.

\begin{tabular}{|l|l|}
\hline & Origin \\
\hline XCalibur 2.2 & Thermo Fisher Scientific, Waltham, USA \\
\hline Proteome Discoverer & Thermo Fisher Scientific, Waltham, USA \\
\hline Geneious R8 & Biomatters Ltd., Auckland, New Zealand \\
\hline analySis 3.2 & Olympus Corporation, Tokyo, Japan \\
\hline ImageJ 1.48 & National Institutes of Health, Bethesda, USA \\
\hline ChemStation & Agilent Technologies, Santa Clara, USA \\
\hline $\begin{array}{l}\text { Basic Local Alignment Search } \\
\text { Tool }\end{array}$ & $\begin{array}{l}\text { National Center for Biotechnology Information } \\
\text { (NCBI), Bethesda, USA (Altschul et al., 1990) }\end{array}$ \\
\hline TargetP 1.1 & $\begin{array}{l}\text { Center for Biological Sequence Analysis, Technical } \\
\text { University of Denmark (Emanuelsson et al., 2000) }\end{array}$ \\
\hline PredAlgo & $\begin{array}{l}\text { Institut de Biologie Physico-Chimique, Paris, France } \\
\text { (Tardif et al., 2012) }\end{array}$ \\
\hline
\end{tabular}




\subsection{Consumables}

Table 3: Consumables used in this study. Listed below are the consumables used in this study, along with the supplier.

\begin{tabular}{|l|l|}
\hline & Supplier \\
\hline CloneJET PCR cloning kit & Thermo Fisher Scientific, Waltham, USA \\
\hline Filtropur S0.2 sterile filter & Sarstedt AG \& Co, Nümbrecht, Germany \\
\hline Fruhstorfer Erde Typ T fein & Hawita Gruppe GmbH, Vechta, Germany \\
\hline GenElute HP Plasmid Midiprep kit & Sigma-Aldrich Co., St. Louis, USA \\
\hline GenElute HP Plasmid Miniprep kit & Sigma-Aldrich Co., St. Louis, USA \\
\hline NucleoSpin Gel and PCR Clean-up kit & Macherey-Nagel, Düren, Germany \\
\hline Protein LoBind 2 mL reaction tube & Eppendorf AG, Hamburg, Germany \\
\hline Silica gel 60 plate & Merck KGaA, Darmstadt, Germany \\
\hline surgical tape & 3M Deutschland GmbH, Neuss, Germany \\
\hline $\begin{array}{l}\text { Takyon No Rox SYBR Core Kit blue } \\
\text { dTTP }\end{array}$ & Eurogentec Biologics Division, Seraing, Belgium \\
\hline
\end{tabular}

\subsection{Chemicals}

All chemicals were obtained from one of the following suppliers, unless stated otherwise:

Merck KGaA, Darmstadt, Germany

Carl Roth GmbH \& Co. KG, Karlsruhe, Germany

Thermo Fisher Scientific, Waltham, USA

Sigma-Aldrich Co., St. Louis, USA 
Table 4: Chemicals used in this study. Listed below are the chemicals used in this study, that were obtained from suppliers other than the ones stated above.

\begin{tabular}{|l|l|}
\hline & Supplier \\
\hline${ }^{14}$ C palmitic acid & $\begin{array}{l}\text { GE Health Care Life Sciences Ltd., Buckinghamshire, } \\
\text { England }\end{array}$ \\
\hline Acetonitrile & Fischer Chemical Co. Ltd., Hong Kong, China \\
\hline Carbenicillin & Duchefa Biochemie B.V, Haarlem, The Netherlands \\
\hline Carboxyl- ${ }^{14}$ C-triolein & PerkinElmer Inc., Waltham, USA \\
\hline Di-17:0 phosphatidylcholine & Larodan Fine Chemicals AB, Limhamn, Sweden \\
\hline Isopropanol & Fischer Chemical Co. Ltd., Hong Kong, China \\
\hline Microagar & Duchefa Biochemie B.V, Haarlem, The Netherlands \\
\hline $\begin{array}{l}\text { Murashige-Skoog salts with } \\
\text { modified vitamins }\end{array}$ & Duchefa Biochemie B.V, Haarlem, The Netherlands \\
\hline Previcur fungicide & Bayer AG, Leverkusen, Germany \\
\hline rifampicin & Duchefa Biochemie B.V, Haarlem, The Netherlands \\
\hline Serva Blue G & Serva Electrophoresis GmbH, Heidelberg, Germany \\
\hline Silwet L-77 silicone surfactant & GE Specialty Materials, Meyrin, Switzerland \\
\hline
\end{tabular}

\subsection{Media and buffers}

\subsubsection{Media}

Unless stated otherwise, media were sterilized by autoclaving at $121^{\circ} \mathrm{C}$ for $20 \mathrm{~min}$.

Table 5: Blue-green algae media (BG11, Stanier et al., 1971)

\begin{tabular}{|l|l|}
\hline & Final concentration [mM] \\
\hline $\mathrm{NaNO}_{3}$ & 17.6 \\
\hline $\mathrm{K}_{2} \mathrm{HPO}_{4}$ & 0.2 \\
\hline $\mathrm{MgSO}_{4}$ & 0.3 \\
\hline $\mathrm{CaCl}_{2}$ & 0.25 \\
\hline $\mathrm{C}_{6} \mathrm{H}_{8} \mathrm{O}_{7}$ & 0.03 \\
\hline Ferric ammonium citrate & 0.02 \\
\hline EDTA & 0.002 \\
\hline $\mathrm{Na}_{2} \mathrm{CO}_{3}$ & 0.19 \\
\hline $\mathrm{H}_{3} \mathrm{BO}_{3}$ & 0.046 \\
\hline $\mathrm{MnCl}_{2}$ & 0.009 \\
\hline $\mathrm{ZnSO}$ & 0.0008 \\
\hline $\mathrm{Na}_{2} \mathrm{MoO}_{4}$ & 0.0016 \\
\hline $\mathrm{CuSO}$ & 0.0003 \\
\hline $\mathrm{Co}\left(\mathrm{NO}_{3}\right)_{2}$ & 0.0002 \\
\hline
\end{tabular}


Table 6: Lysogeny broth (LB media, Bertani, 1951)

\begin{tabular}{|l|l|}
\hline & Final concentration \\
\hline Peptone & $1 \%(\mathrm{w} / \mathrm{v})$ \\
\hline Yeast extract & $0.5 \%(\mathrm{w} / \mathrm{v})$ \\
\hline $\mathrm{NaCl}$ & $1 \%(\mathrm{w} / \mathrm{v})$ \\
\hline
\end{tabular}

$1.5 \%$ (w/v) microagar (Duchefa Biochemie B.V, Haarlem, The Netherlands) was added before autoclaving for LB agar plates.

Table 7: Pollen tube growth media (PT media, Read et al., 1993)

\begin{tabular}{|l|l|}
\hline & Final concentration \\
\hline Sucrose & $5 \%(\mathrm{w} / \mathrm{v})$ \\
\hline Polyethylene glycol (PEG)-6000 & $12.5 \%(\mathrm{w} / \mathrm{v})$ \\
\hline $\begin{array}{l}\text { 2-(N-morpholino)ethanesulfonic acid } \\
\text { (MES)-KOH (pH 5.9) }\end{array}$ & $15 \mathrm{mM}$ \\
\hline $\mathrm{CaCl}$ & $1 \mathrm{mM}$ \\
\hline $\mathrm{KCl}$ & $1 \mathrm{mM}$ \\
\hline $\mathrm{MgSO}_{4}$ & $0.8 \mathrm{mM}$ \\
\hline $\mathrm{H}_{3} \mathrm{BO}_{3}$ & $1.6 \mathrm{mM}$ \\
\hline $\mathrm{CuSO}_{4}$ & $30 \mu \mathrm{M}$ \\
\hline
\end{tabular}

PT media was filter sterilized using a Filtropur S0.2 sterile filter (Sarstedt AG \& Co, Nümbrecht, Germany).

Table 8: 1/2 Murashige-Skoog media (1/2 MS media, Murashige and Skoog, 1962)

\begin{tabular}{|l|l|}
\hline & Final concentration \\
\hline Murashige-Skoog salts with modified vitamins & $0.22 \%(\mathrm{w} / \mathrm{v})$ \\
\hline sucrose & $1 \%(\mathrm{w} / \mathrm{v})$ \\
\hline
\end{tabular}

$\mathrm{pH}$ was adjusted to 5.9 using $0.5 \mathrm{M} \mathrm{KOH} .0 .7 \%(\mathrm{w} / \mathrm{v})$ microagar was added for $1 / 2 \mathrm{MS}$ plates. 
3.5.2 Buffers for cell disruption

Table 9: Centrifugation buffers for LB isolation from L. incisa (modified from Feussner and Kindl, 1992; Sturm et al., 1985)

\begin{tabular}{|l|l|}
\hline & Final concentration \\
\hline Sucrose & 0.6 or $0.4 \mathrm{M}$ \\
\hline Tris- $\mathrm{HCl}(\mathrm{pH} 7.5)$ & $100 \mathrm{mM}$ \\
\hline $\begin{array}{l}\text { Ethylenediaminetetraacetic acid } \\
\text { (EDTA) }\end{array}$ & $3 \mathrm{mM}$ \\
\hline Dithiotreitol (DTT) & $10 \mathrm{mM}$ \\
\hline Plant protease inhibitor cocktail & $1 \%(\mathrm{v} / \mathrm{v})$ \\
\hline
\end{tabular}

DT'T and plant protease inhibitors were added shortly before use.

Table 10: Grinding buffer for disruption of A. thaliana seedlings (modified from Hills and Murphy, 1988)

\begin{tabular}{|l|l|}
\hline & Final concentration \\
\hline sucrose & $0.4 \mathrm{M}$ \\
\hline $\begin{array}{l}\text { 4-(2-hydroxyethyl)-1-piperazineethanesulfonic acid } \\
\text { (HEPES) }\end{array}$ & $100 \mathrm{mM}$ \\
\hline $\mathrm{KCl}$ & $10 \mathrm{mM}$ \\
\hline $\mathrm{MgCl}_{2}$ & $1 \mathrm{mM}$ \\
\hline EDTA & $1 \mathrm{mM}$ \\
\hline DTT & $10 \mathrm{mM}$ \\
\hline Plant protease inhibitor cocktail & $1 \%(\mathrm{v} / \mathrm{v})$ \\
\hline
\end{tabular}

DTT and plant protease inhibitors were added shortly before use. 


\subsubsection{Buffers for SDS-PAGE}

Table 11: Denaturing protein solubilization buffer (modified from Laemmli, 1970; Zienkiewicz et al., 2014)

\begin{tabular}{|l|l|}
\hline & Final concentration \\
\hline Sodium dodecyl sulfate (SDS) & $4 \%(\mathrm{w} / \mathrm{v})$ \\
\hline DTT & $4 \mathrm{mM}$ \\
\hline Glycerol & $8 \%(\mathrm{v} / \mathrm{v})$ \\
\hline Tris-HCl, pH 6.8 & $80 \mathrm{mM}$ \\
\hline Bromophenolblue & $0.02 \%(\mathrm{w} / \mathrm{v})$ \\
\hline urea & $7 \mathrm{M}$ \\
\hline thiourea & $2 \mathrm{M}$ \\
\hline
\end{tabular}

Table 12: Running buffer for SDS-PAGE (Davis, 1964; Laemmli, 1970; Ornstein, 1964)

\begin{tabular}{|l|l|}
\hline & Final concentration \\
\hline Tris-HCl & $25 \mathrm{mM}$ \\
\hline Glycine & $200 \mathrm{mM}$ \\
\hline SDS & $0.1 \%(\mathrm{w} / \mathrm{v})$ \\
\hline
\end{tabular}

Table 13: Running gel for denaturing SDS-PAGE (Davis, 1964; Laemmli, 1970; Ornstein, 1964)

\begin{tabular}{|l|l|}
\hline & Final concentration \\
\hline Tris-HCl, $\mathrm{pH} 8.8$ & $375 \mathrm{mM}$ \\
\hline SDS & $0.1 \%(\mathrm{w} / \mathrm{v})$ \\
\hline Acrylamide/Bis-acrylamide & $10 \% / 0.027 \%(\mathrm{w} / \mathrm{v})$ \\
\hline Ammonium persulfate (APS) & $0.05 \%(\mathrm{w} / \mathrm{v})$ \\
\hline Tetramethylethylenediamine (TEMED) & $0.0007 \%(\mathrm{v} / \mathrm{v})$ \\
\hline
\end{tabular}

Table 14: Stacking gel for denaturing SDS-PAGE (Davis, 1964; Laemmli, 1970; Ornstein, 1964)

\begin{tabular}{|l|l|}
\hline & Final concentration \\
\hline Tris-HCl, pH 6.8 & $125 \mathrm{mM}$ \\
\hline SDS & $0.1 \%(\mathrm{w} / \mathrm{v})$ \\
\hline Acrylamide/Bis-acrylamide & $4 \% / 0.1 \%(\mathrm{w} / \mathrm{v})$ \\
\hline Ammonium persulfate (APS) & $0.05 \%(\mathrm{w} / \mathrm{v})$ \\
\hline Tetramethylethylenediamine (TEMED) & $0.0007 \%(\mathrm{v} / \mathrm{v})$ \\
\hline
\end{tabular}


Table 15: Coomassie staining solution (Meyer and Lamberts, 1965; Neuhoff et al., 1988)

\begin{tabular}{|l|l|}
\hline & Final concentration \\
\hline Serva Blue G & $0.002 \%(\mathrm{w} / \mathrm{v})$ \\
\hline Ethanol & $10 \%(\mathrm{v} / \mathrm{v})$ \\
\hline Acetic acid & $5 \%(\mathrm{v} / \mathrm{v})$ \\
\hline
\end{tabular}

3.5.4 Buffers for nucleic acid extraction

Table 16: Trizol (Chomcyzynski and Sacchi, 1987)

\begin{tabular}{|l|l|}
\hline & Final concentration \\
\hline Guanidinium thiocyanate & $0.8 \mathrm{M}$ \\
\hline Ammonium thiocyanate & $0.4 \mathrm{M}$ \\
\hline Sodium acetate, pH 5 & $0.1 \mathrm{M}$ \\
\hline Glycerol & $5 \%(\mathrm{v} / \mathrm{v})$ \\
\hline Aqueous phenol & $38 \%(\mathrm{v} / \mathrm{v})$ \\
\hline
\end{tabular}

Table 17: High salt precipitation buffer for RNA (Chomcyzynski and Sacchi, 1987)

\begin{tabular}{|l|l|}
\hline & Final concentration \\
\hline Sodium citrate & $0.8 \mathrm{M}$ \\
\hline Sodium chloride & $1.2 \mathrm{M}$ \\
\hline
\end{tabular}

High salt precipitation buffer was sterilized by autoclaving at $121^{\circ} \mathrm{C}$ for $20 \mathrm{~min}$.

Table 18: Seed RNA extraction buffer (Oñate-Sánchez and Vicente-Carbajosa, 2008)

\begin{tabular}{|l|l|}
\hline & Final concentration \\
\hline LiCl & $0.4 \mathrm{M}$ \\
\hline Tris, $\mathrm{pH} 8$ & $0.2 \mathrm{M}$ \\
\hline EDTA, pH 8 & $25 \mathrm{mM}$ \\
\hline SDS & $1 \%(\mathrm{w} / \mathrm{v})$ \\
\hline
\end{tabular}


Table 19: CTAB buffer for extraction of genomic DNA (Weigel and Glazebrook, 2002)

\begin{tabular}{|l|l|}
\hline & Final concentration \\
\hline Cetyltrimethylammoniumbromide (CTAB) & $2 \%(\mathrm{w} / \mathrm{v})$ \\
\hline Tris-HCl, $\mathrm{pH} 8$ & $100 \mathrm{mM}$ \\
\hline EDTA, pH 8 & $20 \mathrm{mM}$ \\
\hline $\mathrm{NaCl}$ & $1.4 \mathrm{M}$ \\
\hline
\end{tabular}

3.5.5 Buffers for agarose gel electrophoresis (Green and Sambrook, 2012)

Table 20: Tris Acetic acid EDTA (TAE) buffer

\begin{tabular}{|l|l|}
\hline & Final concentration \\
\hline Tris & $40 \mathrm{mM}$ \\
\hline Acetic acid & $20 \mathrm{mM}$ \\
\hline EDTA & $1 \mathrm{mM}$ \\
\hline
\end{tabular}

Table 21: 6 x DNA loading dye

\begin{tabular}{|l|l|}
\hline & Final concentration \\
\hline Bromophenol Blue sodium salt & $0.25 \%(\mathrm{w} / \mathrm{v})$ \\
\hline Xylene cyanol & $0.25 \%(\mathrm{w} / \mathrm{v})$ \\
\hline Glycerol & $30 \%(\mathrm{v} / \mathrm{v})$ \\
\hline EDTA & $10 \mathrm{mM}$ \\
\hline
\end{tabular}

3.5.6 TFP buffer for generation of competent E. coli

Table 22: TFP buffer

\begin{tabular}{|l|l|}
\hline $\begin{array}{l}\text { piperazine-N,N'-bis(2-ethanesulfonic acid) } \\
\text { (PIPES) }\end{array}$ & Final concentration \\
\hline $\mathrm{CaCl}_{2}$ & $10 \mathrm{mM}$ \\
\hline $\mathrm{KCl}$ & $15 \mathrm{mM}$ \\
\hline $\mathrm{MnCl}_{2}$ & $250 \mathrm{mM}$ \\
\hline
\end{tabular}


3.5.7 Tris EDTA buffer (TE buffer) for Gateway cloning

Table 23: TE buffer

\begin{tabular}{|l|l|}
\hline & Final concentration \\
\hline Tris-HCl & $10 \mathrm{mM}$ \\
\hline EDTA & $1 \mathrm{mM}$ \\
\hline
\end{tabular}

3.5.8 Solutions for lipid extraction

Table 24: Fatty acid methyl ester (FAME) solution for acidic methanolysis (Miquel and Browse, 1992)

\begin{tabular}{|l|l|}
\hline & Final concentration \\
\hline Methanol & $63.7 \%(\mathrm{v} / \mathrm{v})$ \\
\hline Toluol & $31.8 \%(\mathrm{v} / \mathrm{v})$ \\
\hline Sulfuric acid & $2.5 \%(\mathrm{v} / \mathrm{v})$ \\
\hline Dimethoxypropane & $2 \%(\mathrm{v} / \mathrm{v})$ \\
\hline
\end{tabular}

3.5.9 Solutions for lipase activity assays

Table 25: Bradford reagent (Bradford, 1976)

\begin{tabular}{|l|l|}
\hline & Final concentration \\
\hline Serva Blue G $(0.14 \% \mathrm{w} / \mathrm{v}$ in ethanol $)$ & $0.007 \%(\mathrm{w} / \mathrm{v})$ \\
\hline Phosphoric acid & $8.5 \%(\mathrm{w} / \mathrm{v})$ \\
\hline
\end{tabular}

Table 26: Buffer for lipase reaction, $\mathrm{pH} 6$

\begin{tabular}{|l|l|}
\hline & Final concentration \\
\hline Citric acid & $0.1 \mathrm{M}$ \\
\hline DTT & $2 \mathrm{mM}$ \\
\hline Plant protease inhibitor cocktail & $1 \%(\mathrm{v} / \mathrm{v})$ \\
\hline
\end{tabular}

$\mathrm{pH}$ was set to 6 using $\mathrm{KOH}$, DTT and Plant protease inhibitors were added just before use. 
Table 27: Buffers for lipase reaction, $p H 7$ and 8

\begin{tabular}{|l|l|}
\hline & Final concentration \\
\hline Tris & $0.1 \mathrm{M}$ \\
\hline DTT & $2 \mathrm{mM}$ \\
\hline Plant protease inhibitor cocktail & $1 \%(\mathrm{v} / \mathrm{v})$ \\
\hline
\end{tabular}

pH was set to 7 or 8 using HCl, DTT and Plant protease inhibitors were added just before use.

\subsection{Organisms}

3.6.1 Algae and plant lines

Lobosphaera incisa strain SAG 2468

Arabidopsis thaliana Columbia-0 and sdp1-5/sdp1-L2 (Kelly et al., 2011)

Nicotiana tabacum SNN

3.6.2 Bacterial strains

Escherichia coli XL1-Blue (Agilent Technologies, Santa Clara, USA)

Escherichia coli DH5- $\alpha$ (New England Biolabs, Ipswich, USA)

Agrobacterium tumefaciens EHA 105 


\subsection{Oligonucleotides}

All oligonucleotides were obtained from Sigma-Aldrich Chemie GmbH, Steinheim, Germany.

Table 28: Oligonucleotides used in this study. Nucleotide sequences are given along with their general purpose and the target coding sequence. Added endonuclease restriction sites are indicated in capital letters.

\begin{tabular}{|c|c|c|}
\hline Target & Primer nucleotide sequence & General purpose \\
\hline \multirow{3}{*}{ g555.t1 } & ataGGATCCatgggtgctgagccagtg & \multirow{29}{*}{$\begin{array}{l}\text { Amplification of coding } \\
\text { sequences from cDNA and } \\
\text { addition of restriction sites }\end{array}$} \\
\hline & ataGTCGACgtgagcgecgttcacctc & \\
\hline & ataGTCGACatgggtgctgagccagtg & \\
\hline \multirow{5}{*}{ g2905.t1 } & ggaGGATCCatgtactcagcgatgcagttg & \\
\hline & ggaGAATTCttagccetgcgggaacct & \\
\hline & ataGTCGACatgtactcagcgatgcagttg & \\
\hline & ataGGATCCttagccctgcgggaacct & \\
\hline & ataGTCGACgecctgcgggaacctggcat & \\
\hline \multirow{7}{*}{ g9192.t1 (LiSDP1) } & atgaaacctctgacttaccaaacgggtcgc & \\
\hline & tcatggcgcgatgacgtccagcgcctggcc & \\
\hline & ggaGGATCCatgaaacctctgacttaccaaac & \\
\hline & ataGTCGACtcatggcgcgatgacgtc & \\
\hline & ataGTCGACatgaaacctctgacttaccaaac & \\
\hline & ataGGATCCtcatggcgcgatgacgtc & \\
\hline & ataGTCGACtggcgcgatgacgtccag & \\
\hline \multirow{2}{*}{ g9582.t1 } & ataGGATCCatgcaagacccgtaccaacg & \\
\hline & ataCTCGAGgggcaaaaacagctggtagt & \\
\hline \multirow{2}{*}{ g13714.t1 } & ataGGATCCatgcgtgccgcagcaagc & \\
\hline & ataCTCGAGtgccgccgctgtcaacgc & \\
\hline \multirow{2}{*}{ g13747.t1 } & agaGGATCCatgactaagtccacctcatccc & \\
\hline & ataGTCGACctgttcagcgctagcggc & \\
\hline \multirow{3}{*}{ g13945.t1 } & ataGGATCCatggcatctcatgacaacctg & \\
\hline & ataCTCGAGtttcatgtttaggccattgctg & \\
\hline & ataACATGTatggcatctcatgacaacctg & \\
\hline \multirow{2}{*}{ g14373.t1 } & ggcCTCGAGatgtctgtccgtcagccg & \\
\hline & ataCCCGGGtgccgctgcagtcgccggc & \\
\hline \multirow{3}{*}{ g15430.t1 } & ataGGATCCatgtataacgcagacgggtccat & \\
\hline & ataGTCGACccatccaaagctgaacgtgc & \\
\hline & ataGTCGACatgtataacgcagacgggtc & \\
\hline mVenus & ataGGATCCttacttgtacagctcgtcca & $\begin{array}{l}\text { Amplification of } \mathrm{C} \text {-terminal } \\
\mathrm{mVenus} \text { fusions and addition } \\
\text { of restriction site }\end{array}$ \\
\hline
\end{tabular}


Table 28

continued

\begin{tabular}{|c|c|c|}
\hline \multirow{2}{*}{ A. thaliana Actin 8} & atggccgatgctgatgacattcaacct & \multirow{6}{*}{$\begin{array}{l}\text { Confirmation of gene } \\
\text { expression }\end{array}$} \\
\hline & ttagaagcattttctgtggacaatgcctg & \\
\hline \multirow{2}{*}{ A. thaliana SDP1 } & accgtttgctccccaagaaa & \\
\hline & cttgagcctccgcatatggt & \\
\hline \multirow{2}{*}{$\begin{array}{l}\text { A. thaliana } \\
\text { SDP1-L }\end{array}$} & tcattcctcggtgggttagc & \\
\hline & tacagaccccacgcttgaac & \\
\hline \multirow{2}{*}{ g555.t1 } & tgtctacagtactggcaagagc & \multirow{12}{*}{ qRT-PCR } \\
\hline & tgttccatactcgctgactgtc & \\
\hline \multirow{2}{*}{ g2905.t1 } & ataccgcttgcgctattctc & \\
\hline & tcacatgcgcaaacacgatg & \\
\hline \multirow{2}{*}{$\begin{array}{l}\text { g5830 (ribosomal } \\
\text { protein S21) }\end{array}$} & caacgtagacaaggccttttcc & \\
\hline & tctccttctggttgagcactc & \\
\hline \multirow{2}{*}{ g9192.t1 (LiSDP1) } & ggtgttgtcaagacgctctttg & \\
\hline & ttggttgcgatgatggatgc & \\
\hline \multirow{2}{*}{ g13945.t1 } & atgctcacaaactcgtggac & \\
\hline & ttgccaatgcccttcttgac & \\
\hline \multirow{2}{*}{ g15430.t1 } & acatctttggcaaccacagc & \\
\hline & atcgctgatgtcacggactc & \\
\hline
\end{tabular}

\subsection{Vectors}

Table 29: Vectors used for cloning and heterologous expression in this study. Listed below are vector names and their origin.

\begin{tabular}{|l|l|}
\hline & Origin \\
\hline pUC18-Entry & $\begin{array}{l}\text { Dr. Ellen Hornung, University of Göttingen (Hornung } e t \\
\text { al., 2005) }\end{array}$ \\
\hline pENTRY-E-napin::MCS & $\begin{array}{l}\text { Dr. Mareike Heilmann, University of Halle, formerly } \\
\text { University of Göttingen (Heilmann } e t \text { al., 2012) }\end{array}$ \\
\hline pCambia 23.1 & $\begin{array}{l}\text { Dr. Ellen Hornung, University of Göttingen } \\
\text { (generated as described previously, Hornung } e t \text { al., 2005) }\end{array}$ \\
\hline pCambia 43.0 & $\begin{array}{l}\text { Dr. Ellen Hornung, University of Göttingen } \\
\text { (generated as described previously, Hornung } \text { et al., 2005) }\end{array}$ \\
\hline pJET1.2/blunt & Thermo Fisher Scientific, Waltham, USA \\
\hline pUC-Lat52::mVenus & $\begin{array}{l}\text { Prof. Dr. Jörg Kudla, University of Münster (Mähs } \text { et al.., } \\
\text { 2013) }\end{array}$ \\
\hline
\end{tabular}

For features of pCambia vectors, see appendix 9.3. 


\subsection{Enzymes}

Table 30: Enzymes used in this study. Listed below are enzyme names and the supplier they were obtained from.

\begin{tabular}{|l|l|}
\hline & Supplier \\
\hline DNaseI & Thermo Fisher Scientific, Waltham, USA \\
\hline GoTaq Polymerase & Promega Corporation, Madison, USA \\
\hline LR Clonase II enzyme mix & Thermo Fisher Scientific, Waltham, USA \\
\hline Phusion Polymerase & New England Biolabs, Ipswich, USA \\
\hline Plant protease inhibitor cocktail & Sigma-Aldrich Co., St. Louis, USA \\
\hline RedTaq Polymerase & Sigma-Aldrich Co., St. Louis, USA \\
\hline restriction enzymes & Thermo Fisher Scientific, Waltham, USA \\
\hline $\begin{array}{l}\text { RevertAid H Minus reverse } \\
\text { transcriptase }\end{array}$ & Thermo Fisher Scientific, Waltham, USA \\
\hline Trypsin & Sigma-Aldrich Co., St. Louis, USA \\
\hline
\end{tabular}




\section{Results}

L. incisa is a unicellular microalga that is of special interest due to its ability to accumulate large amounts of the PUFA ARA in LBs. In this study, lipid and protein analyses were carried out in order to elucidate the biogenesis of LBs in conditions of stress and recovery.

\subsection{Analysis of physiological responses to changes in nitrogen availability}

Physiological responses of L. incisa strain SAG2468 were investigated during a $7 \mathrm{~d}$ period of nitrogen limitation followed by $3 \mathrm{~d}$ in nitrogen replete conditions.

\subsubsection{Determination of pigment and fatty acid content}

The ratio of carotenoids to total chlorophyll is a useful indication of the physiological state of L. incis a cultures (Solovchenko et al., 2009). The ratio increases during the first $3 \mathrm{~d}$ of nitrogen limitation when it reaches a plateau and slightly increases once more upon nitrogen resupply before reaching a basal level (Figure 1). This pattern is also found in TAG fatty acids reaching $56 \%$ of the total after the first $3 \mathrm{~d}$, albeit the increase observed $1 \mathrm{~d}$ after nitrogen resupply is more pronounced with a maximum of $90 \%$ of the total (Figure 1, Supplemental figure 1). Total fatty acids (TFAs) do not undergo significant changes during the $7 \mathrm{~d}$ in growth limiting conditions, whereas they decrease when nitrogen is resupplied. The proportion of fatty acids sequestered in TAG molecules increases slightly to approximately $50 \%$ during the first part of the experiment, however it is strongly affected by the change of growth media at day 7 , increasing to approximately $90 \%$ during $1 \mathrm{~d}$.

Nitrogen supply not only affects the total amount but also the profile of all fatty acids (Figure 2) and those esterified in TAG (Figure 3). Comparing proportions in TFAs (Figure 2A), the saturated fatty acid species 16:0 and 18:0 show opposite trends over the course of the experiment: The relative amount of 16:0 is reduced after $3 \mathrm{~d}$ and only recovers to the initial level in the second half of the experiment, whereas 18:0, a minor species in L. incisa, doubles in relative amount during $7 \mathrm{~d}$ of nitrogen depletion and is diminished in the second half of the experiment. Neither of the two species is affected in the relative amount found in TAG (Figure $3 \mathrm{~A})$. 


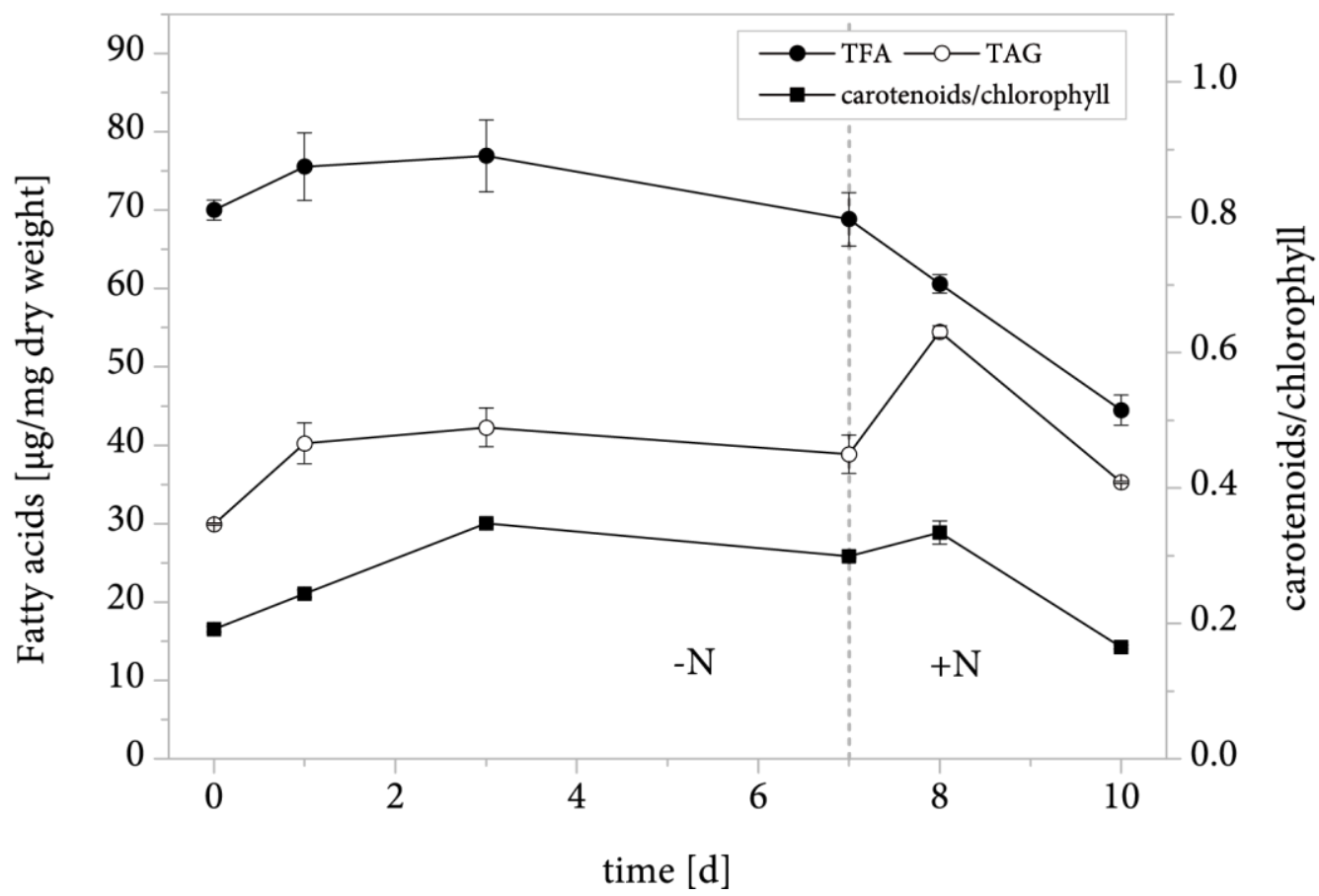

Figure 1: Response of L. incisa to a sequence of nitrogen deplete and replete conditions. The TFA content and the amount of fatty acids in TAG as well as the ratio of carotenoids to total chlorophyll are shown. Error bars represent the standard error of the mean of three L. incisa batches cultivated in parallel in a single experiment.

In L. incisa, two monounsaturated fatty acid species can be detected: 18:1(n-9) and 18:1(n-7). The former undergoes the most dramatic increase in response to nitrogen starvation out of all fatty acids analyzed (Figure 2A), reaching a maximum of $27 \%$ of TFAs within $3 \mathrm{~d}$ and decreasing to its initial level of $17 \%$ in the presence of nitrate. The latter does not appear to be affected by the changing growth conditions in this experiment.

L. incisa is rich in PUFAs, particularly ARA (20:4 (n-6)), as shown in Figure 2B. 18:3 (n-6) and 20:5 (n-3) are of relatively low abundance and do not undergo significant changes. 18:2 (n-6) is the only fatty acid observed in this experiment that decreases in relative abundance but then rises to its initial proportion within the first $7 \mathrm{~d}$. The relative amount of 18:3 (n-3) is strongly reduced from 12 to $2 \%$ of TFAs and recovers completely within $3 \mathrm{~d}$ of nitrogen resupply. The same trend can be observed in TAG, however 18:3 (n-3) is only a minor species in this lipid (Figure 3B). ARA strongly accumulates in response to nitrogen starvation, accounting for almost $30 \%$ of all fatty acids at day 3 of nitrogen limitation, and remains largely unchanged afterwards. Interestingly, the proportion of ARA in TAG follows 
this trend in reverse order, so that no significant change can be observed under nitrogen limiting conditions, in contrast to a clear accumulation upon nitrogen resupply.

A

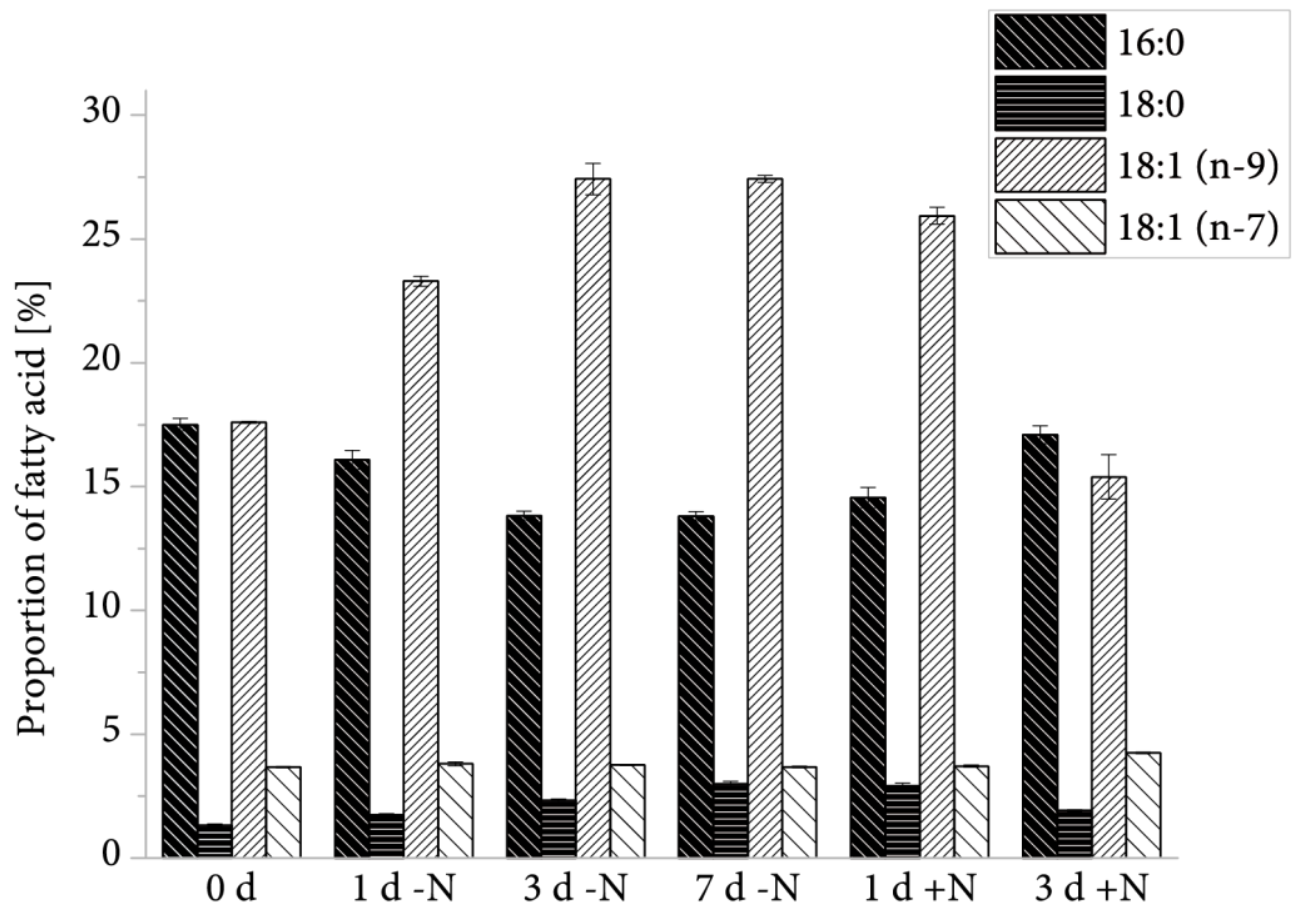

B

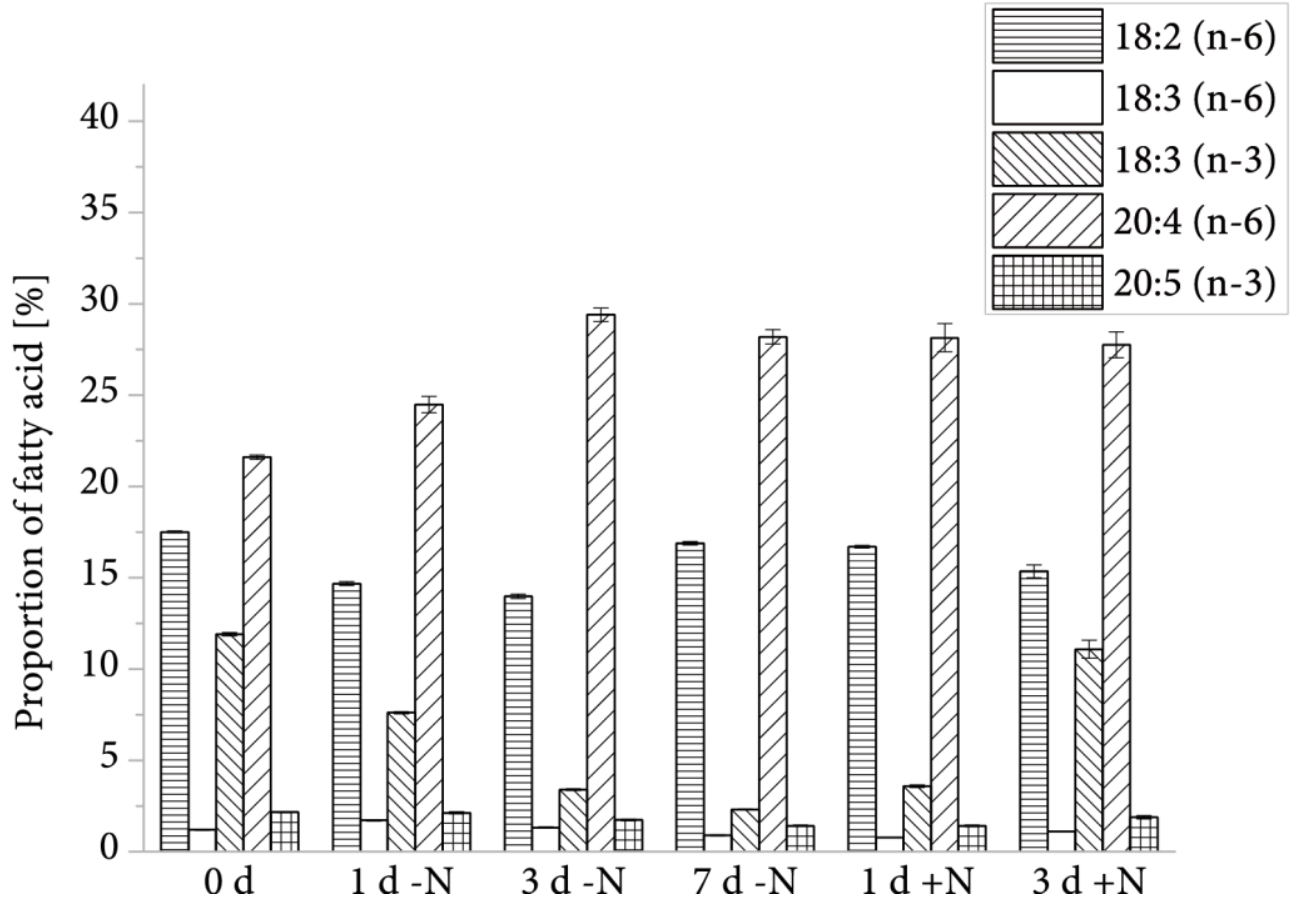

Figure 2: Effect of nitrogen starvation and recovery on the TFA profile of L. incisa. For each fatty acid species, the proportion relative to the total of fatty acids is shown and error bars represent the standard error of the mean of three $L$. incis a batches cultivated in parallel in a single experiment. A, Saturated and monounsaturated fatty acids. B, PUFAs. 
Combined, nitrogen depletion leads to a reduction in C16 fatty acids in favor of C20 species, while the overall C18 content is not affected. Furthermore, the accumulation of TAG in L. incisa during cultivation in nitrogen deplete conditions can be largely attributed to an increase in 18:1 (n-9). This shift in the TAG fatty acid profile is not reflected in the distribution

A

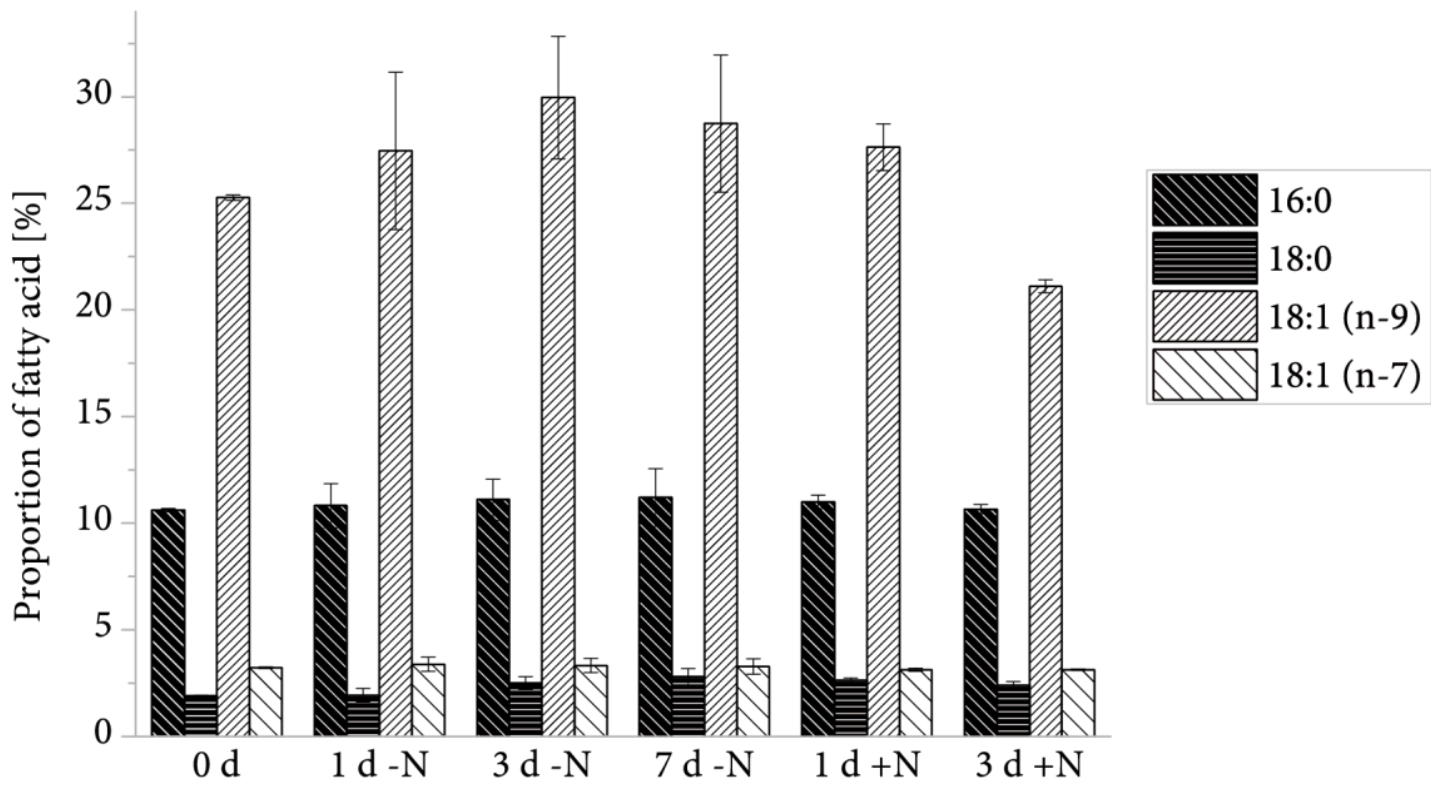

B

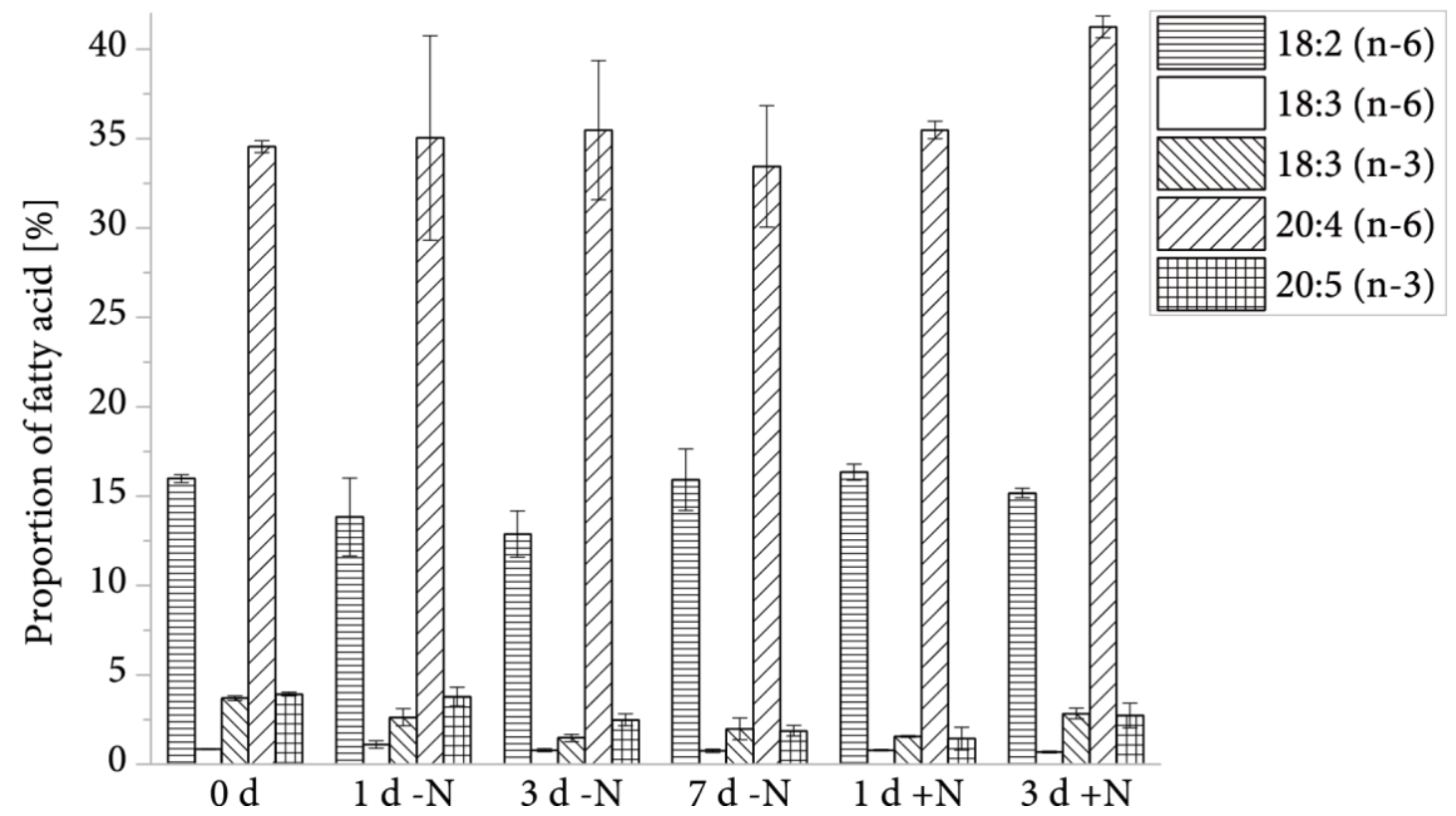

Figure 3: Effect of nitrogen starvation and recovery on the TAG fatty acid profile of L. incisa. For each fatty acid species, the proportion relative to the total of fatty acids in TAG is shown and error bars represent the standard error of the mean of three L. incisa batches cultivated in parallel in a single experiment. A, Saturated and monounsaturated fatty acids. B, PUFAs. 
of TFAs, since the change in the monounsaturated fatty acid is outweighed by reductions in 16:0 and 18:3 (n-3). Additionally, the ratio of ARA and 16:0 is altered in lipid classes other than TAG in this phase of the experiment.

Following nitrogen resupply, the decrease in TFAs can be mostly attributed to 18:1 (n-9) being removed from TAG, while ARA accumulation alone leads to a TAG increase.

\subsubsection{Gene expression analyses}

In order to analyze the physiological changes in L. incisa on the transcript level, the identification of an appropriate housekeeping gene to be used as a reference for normalization was necessary. The alga goes into growth arrest when nitrogen is not available and resumes growth upon resupply of nitrogen (Cheng-Wu et al., 2002), which is a common response in microalgae and known to be associated with extensive changes in the transcriptome (Blaby et al., 2013; Miller et al., 2010; Schmollinger et al., 2014). Many housekeeping genes are likely to be affected by these global changes in gene expression and may therefore not be suitable as references.

Initially, the published results of a set of RNA sequencing (RNAseq) experiments with C. reinhardtii (Tsai et al., 2014) were used to identify genes exhibiting constitutive expression independently of cell division. The authors had sequenced the transcripts of $C$. reinhardtii wildtype and the cht 7 mutant, which is unable to resume growth after nitrogen resupply due to a defect in a central regulator of cellular quiescence. Transcriptomes of cultures growing in normal conditions or subjected to $48 \mathrm{~h}$ nitrogen starvation had been sequenced and for each treatment, the authors compared the expression changes between the genotypes. For the purpose of this study, genes with stable expression across both genotypes and treatments $(\log 2$ of expression changes: -0.1 to 0.1 ) were selected and homologs in L. incisa were identified by BLAST search. The candidate genes were then checked for stable expression in L. incisa following 12 or $72 \mathrm{~h}$ nitrogen starvation (unpublished RNA sequencing data) and several candidates were selected for quantitative real-time PCR (qRT-PCR) analysis. Samples from one culture that was part of the growth experiment described in section 4.1.1 were used for RNA extraction with additional samples taken $6 \mathrm{~h}$ after each change in growth media. The results of the qRT-PCR for RNA HELICASE (transcript accession g5223.t1, homologous to C. reinhardtii Cre01.g051550), POLY(A) BINDING PROTEIN (g11952.t2, Cre17.g725300), NUCLEAR MATRIX PROTEIN (g4250.t2, Cre16.g695300) and a SPLICEOSOME COMPONENT (g7962.t1, Cre01.g051100) relative to time point 0 for three biological replicates 
are shown in Figure 4. All candidates tested exhibited a marked reaction to the changes in nitrogen availability at the time points that were not covered by either RNAseq experiment. An additional gene was thus tested that exhibits only minor alterations in expression ( $\log 2$ of expression changes: -0.1 to 0.1 ) in L. incisa cultures at 0,12 and $72 \mathrm{~h}$ nitrogen starvation (unpublished RNAseq data). The gene putatively encodes RIBOSOMAL PROTEIN S21 (L. incisa transcript accession g5830.t1) and is the most stably expressed one out of all candidates. It was therefore used as a reference gene for normalization in all further qRT-PCR experiments.

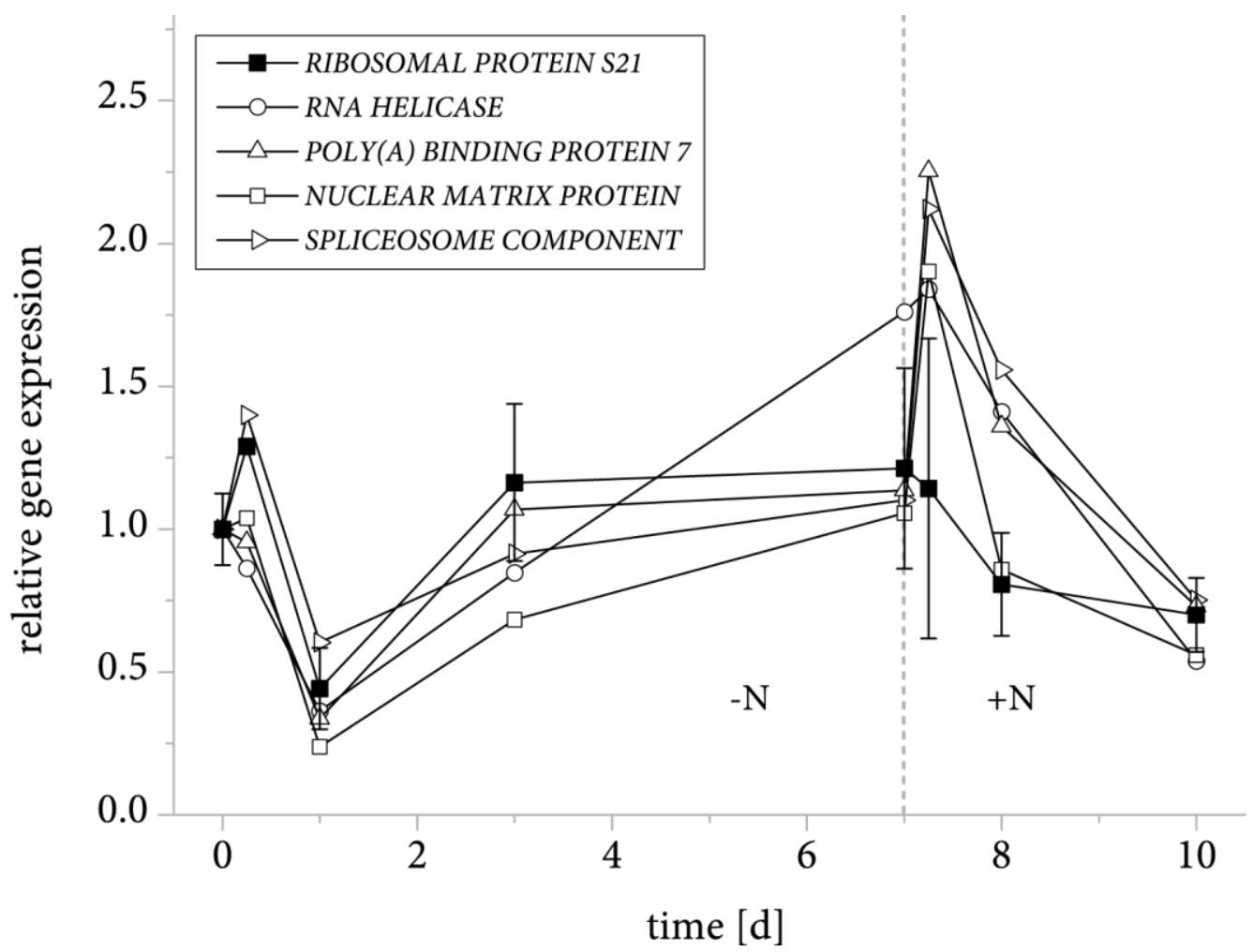

Figure 4: Quantitative real-time PCR measurements of various housekeeping genes in response to changes in nitrogen supply. Transcript levels are shown relative to time point 0 and for the RIBOSOMAL PROTEIN S21 gene error bars represent the standard error of the mean for three batches cultivated in parallel in a single experiment. All other genes were tested using complementary DNA from one algal culture from the same experiment. The dotted line indicates nitrogen resupply. 


\subsection{Identification of L. incisa LB proteins}

In many organisms, proteins are attached to the surface of LBs which can play a role in maintaining the structural integrity of the organelle or actively participate in lipid metabolism.

The L. incisa genome (unpublished data) was therefore searched for homologs of genes encoding known LB proteins. Oleosins, caleosins and steroleosins found in many oil seed plants were used as queries in BLAST searches along with mammalian perilipins and algal proteins including C. reinhardtii Major Lipid Droplet Protein and the Lipid Droplet Surface Protein conserved in Nannochloropsis species. None of these queries yielded any homologies, consequently LBs were isolated from L. incisa in order to identify new LB proteins.

An L. incisa culture that had been starved of nitrogen for $3 \mathrm{~d}$ in order to promote the formation of LBs was used for LB isolation (see

Figure $5 \mathrm{~A}$ and $\mathrm{B}$ ) and a range of cell disruption methods was tested to obtain intact LBs from the alga with as little contamination from other membranes as possible. This proved to be challenging as L. incisa has an especially robust cell wall compared to other microalgae that have been used for this type of analysis in the past. Mechanical cell disruption was attempted using a Potter-Elvehjem tissue grinder, glass beads, pipetting, exposure to ultrasound using a sonification bath and a sonifier tip. In addition to this, a mix of cell wall degrading enzymes frequently used for the generation of A. thaliana protoplasts, consisting of cellulase, hemicellulase and pectinase, was tested on L. incisa cells alone and in combination with the Potter-Elvehjem tissue grinder. Efficient cell disruption was not achieved with any of these methods. A rapid pressure drop using a French press set to pressures up to 3000psi proved equally unsuccessful, whereas 4000psi were sufficient to break the algal cells, however intact LBs could not be retrieved from these cell extracts (data not shown). Cells were finally disrupted
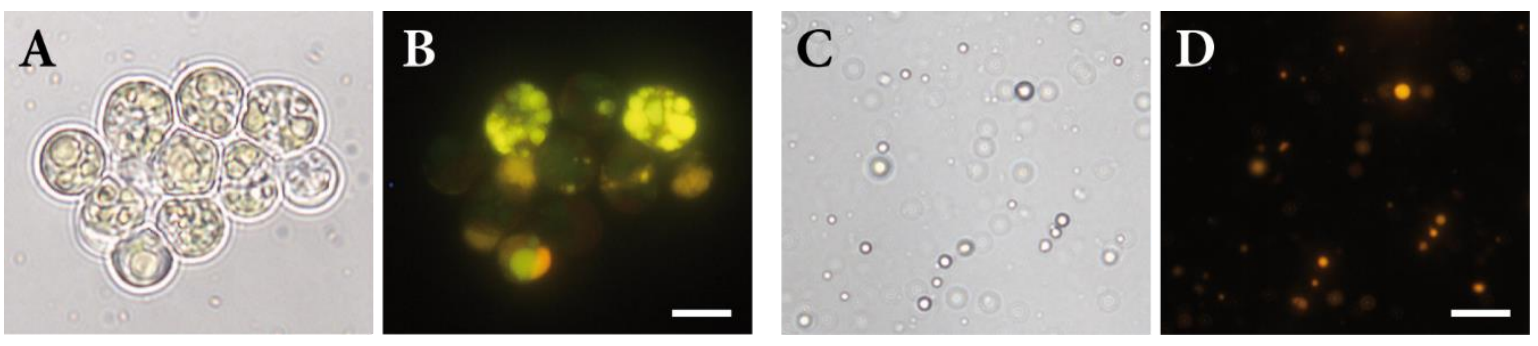

Figure 5: Detection of LBs with Nile Red. Scale bars $=10 \mu \mathrm{m} . A$ and B, Brightfield and fluorescence images of $L$. incis a cells starved of nitrogen for $3 d$. C and D, Brightfield and fluorescence images of $L B S$ that were isolated from $L$. incisa and used for protein extraction. 
by grinding in liquid nitrogen and intact LBs were isolated as described in section 2.2 (see Figure 5 C and D).

Proteins isolated from the LB sample were identified by mass spectrometry, yielding a list of 4105 proteins that were identified by more than one detected peptide with at least medium confidence. This list of putatively LB-associated proteins was compared to proteins identified that way in total protein extract, soluble and microsome fractions obtained from the same algal sample. Table 31 shows an excerpt from the list of identified proteins including the proteins that were characterized in more detail in this study. Among the most abundant proteins, several ones can be clearly assigned to other cellular compartments based on sequence homology with known proteins, such as VOLTAGE-DEPENDENT ANION CHANNEL PROTEIN 2 (VDAC2), ATP SYNTHASE CF1 SUBUNIT $\beta$ (ATP $\beta$ ), PHOTOSYSTEM II OXYGENEVOLVING ENHANCER PROTEIN (PSBQ) and LIGHT-HARVESTING COMPLEX II CHLOROPHYLL A/B BINDING PROTEIN 2 (LHCB2). Proteins were selected for further analysis based on the criteria outlined in section 2.3.4 and the coding sequences that could be successfully amplified from cDNA were studied in more detail. 
Table 31: Proteins identified in L. incisa LB samples. Only proteins that were detected in all three technical replicates are shown. \# denotes the rank in abundance as determined based on the protein's average Normalized Spectral Abundance Factor (NSAF) in LB protein extracts. Coverage refers to the proportion of the amino acid sequence covered by peptides detected in all samples. SD, standard deviation of three technical replicates in a single experiment; VDAC2, voltage-dependent anion channel protein 2; ATP $\beta$, ATP synthase CF1 subunit $\beta$; PSBQ, Photosystem II oxygen-evolving enhancer protein; LHCB2, light-harvesting complex II chlorophyll a/b binding protein 2; LiOGP, L. incisa Oil Globule Protein.

\begin{tabular}{|c|c|c|c|c|c|c|}
\hline \multirow{2}{*}{$\begin{array}{c}\text { Protein } \\
\text { name } \\
\text { (accession) }\end{array}$} & \multirow{2}{*}{$\begin{array}{c}\text { Average NSAF } \\
\quad \pm \text { SD }\end{array}$} & \multirow[b]{2}{*}{$\#$} & \multirow{2}{*}{$\begin{array}{c}\text { Coverage } \\
{[\%]}\end{array}$} & \multicolumn{3}{|c|}{$\begin{array}{c}\begin{array}{c}\text { Fold enrichment compared } \\
\text { to }\end{array} \\
\end{array}$} \\
\hline & & & & $\begin{array}{c}\text { Total } \\
\text { protein } \\
\text { extract }\end{array}$ & $\begin{array}{l}\text { Micro- } \\
\text { somes }\end{array}$ & $\begin{array}{l}\text { Soluble } \\
\text { protein }\end{array}$ \\
\hline g12938.p1 & $0.01333 \pm 0.0005$ & 1 & 84.5 & 1.50 & 1.49 & 1.71 \\
\hline $\begin{array}{l}\text { VDAC2 } \\
(\mathrm{g} 14145 . \mathrm{p} 1)\end{array}$ & $0.00988 \pm 0.0006$ & 2 & 84.0 & 2.02 & 2.58 & 3.03 \\
\hline $\begin{array}{l}\text { ATP } \beta \\
\text { (atpB.p1) }\end{array}$ & $0.00754 \pm 0.0018$ & 3 & 92.8 & 1.16 & 2.62 & 1.39 \\
\hline g15892.p4 & $0.00749 \pm 0.0019$ & 4 & 67.5 & 7.09 & 9.67 & 10.82 \\
\hline g15892.p2 & $0.00748 \pm 0.0019$ & 5 & 72.5 & 7.15 & 10.21 & 11.20 \\
\hline g5834.p2 & $0.00671 \pm 0.0003$ & 6 & 87.9 & 2.41 & 6.55 & 3.26 \\
\hline g5834.p1 & $0.00666 \pm 0.0004$ & 7 & 87.8 & 2.39 & 6.46 & 3.32 \\
\hline $\begin{array}{l}\text { PSBQ } \\
(\mathrm{g} 4252 . p 1)\end{array}$ & $0.00656 \pm 0.0030$ & 8 & 53.9 & 1.67 & 2.10 & 2.89 \\
\hline $\begin{array}{l}\text { LHCB2 } \\
(\mathrm{g} 4499 . \mathrm{p} 1)\end{array}$ & $0.00642 \pm 0.0020$ & 9 & 77.8 & 0.73 & 1.05 & 0.89 \\
\hline g14820.p1 & $0.00642 \pm 0.0002$ & 10 & 58.6 & 2.79 & 1.22 & 2.56 \\
\hline \multicolumn{7}{|c|}{$\vdots$} \\
\hline $\begin{array}{l}\text { LiOGP } \\
\text { (g555.p1) }\end{array}$ & $0.00494 \pm 0.0001$ & 28 & 75.5 & 1.04 & 1.55 & 0.95 \\
\hline \multicolumn{7}{|c|}{$\vdots$} \\
\hline g13945.p1 & $0.00454 \pm 0.0010$ & 36 & 49.1 & 5.48 & 7.32 & 9.18 \\
\hline \multicolumn{7}{|c|}{$\vdots$} \\
\hline g15430.p1 & $0.00320 \pm 0.0014$ & 62 & 64.8 & 31.35 & $*$ & 26.57 \\
\hline \multicolumn{7}{|c|}{ 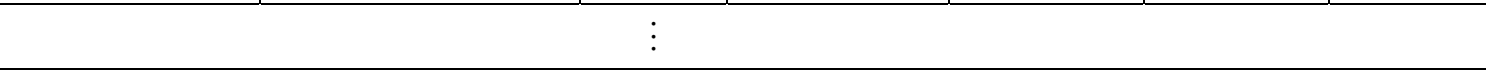 } \\
\hline g13747.p1 & $0.00117 \pm 0.0002$ & 214 & 37.0 & 7.99 & 25.70 & 12.8 \\
\hline
\end{tabular}




\subsubsection{Characterization of L. incisa OIL GLOBULE PROTEIN (LiOGP)}

The protein encoded by the $g 555$ gene was identified as a putative structural LB protein based on its relatively high abundance in LB protein extract (see Table 31) as well as the fact that the protein was found to exhibit some homology with a known LB associated protein from the unicellular green alga Haematococcus pluvialis. The protein was thus termed L. incisa OIL GLOBULE PROTEIN (LiOGP) based on homology with H. pluvialis OIL GLOBULE PROTEIN (HpOGP, see Figure 6A). $75.5 \%$ of the LiOGP amino acid sequence is covered by tryptic peptides detected in the mass spectrometry experiment as shown in Table 31 and Figure 6A. Hydrophobicity of the amino acid sequences follows a highly similar pattern (Figure 6B) and it is notable that neither protein contains a distinct hydrophobic stretch as the one found in oleosins of higher plants (A. thaliana OLEOSIN1 is shown for comparison). No conserved domains collected in the PFAM database were detected and structural homologies to other proteins could not be found using the PHYRE2 server.

The subcellular localization of the protein was confirmed by heterologous expression of a gene fusion with mVenus in $N$. tabacum pollen tubes (

Figure 7). The fluorescence emitted by the mVenus tag shows a distinct punctate pattern typical of LBs (

Figure 7A) and overlaps with the neutral lipid stain Nile Red (

Figure 7B).

In order to gain more information on the function of LiOGP, gene expression was analyzed in L. incisa cultures subjected to nitrogen starvation followed by a change to nitrogen replete media as described in section 4.1.2. Transcript levels closely track the accumulation of TAG during $7 \mathrm{~d}$ of nitrogen limitation and decrease to the initial value during recovery with a local minimum reached $6 \mathrm{~h}$ after the change in growth media (Figure 8). 
A

\begin{tabular}{|c|c|c|}
\hline $\begin{array}{l}\text { LiOGP } \\
\text { HpOGP }\end{array}$ & $\begin{array}{l}1 \\
1\end{array}$ & 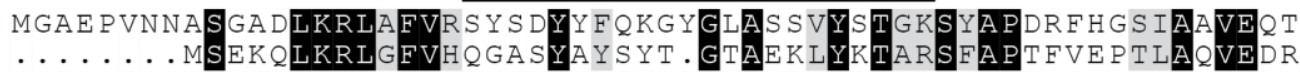 \\
\hline $\begin{array}{l}\text { IOGP } \\
\mathrm{pOGP}\end{array}$ & $\begin{array}{l}180 \\
167\end{array}$ & 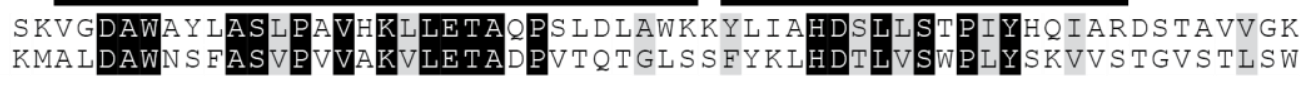 \\
\hline
\end{tabular}

B

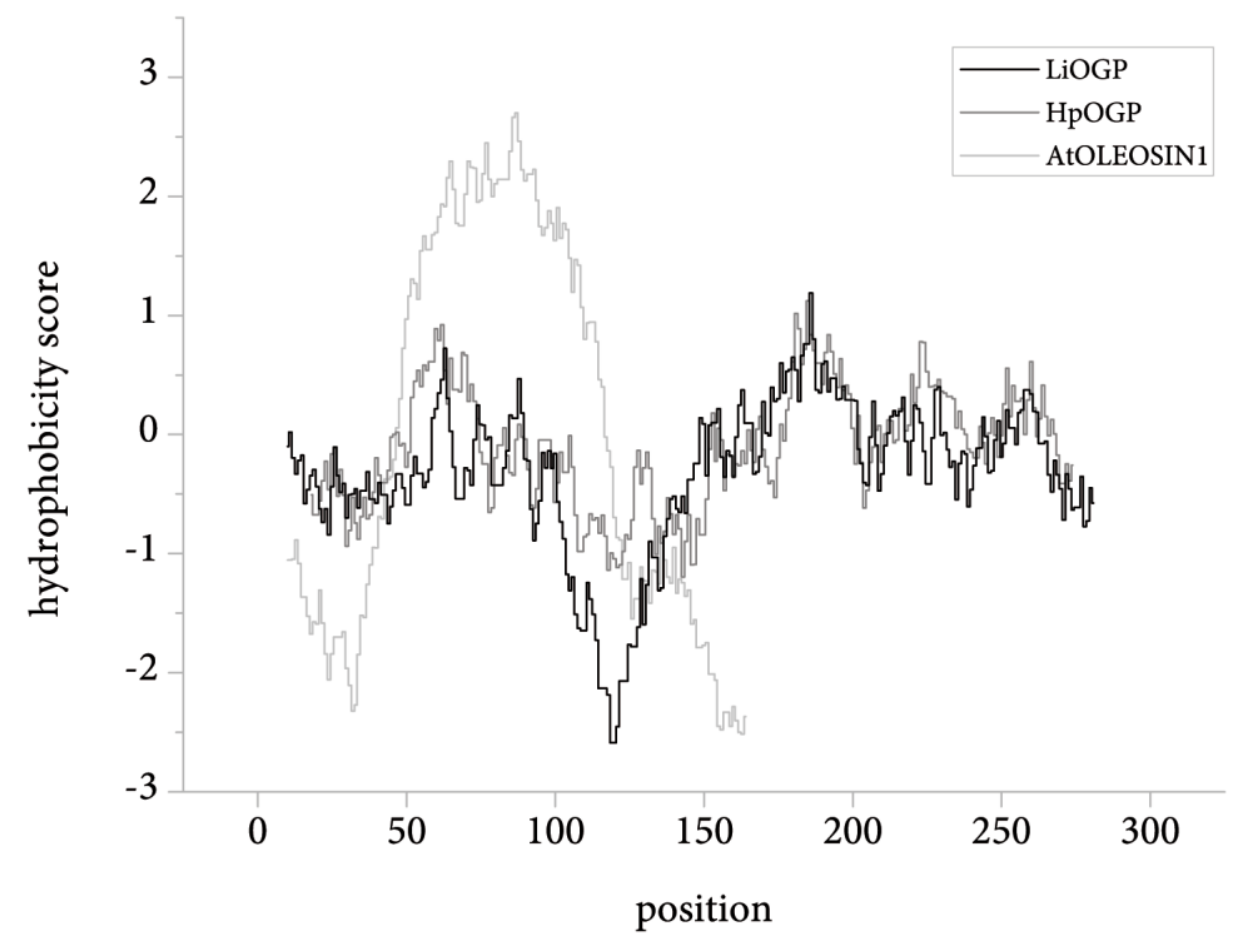

Figure 6: Features of the L. incis a OIL GLOBULE PROTEIN (LiOGP) amino acid sequence. A, Similarities with the amino acid sequence of Haematococcus pluvialis OIL GLOBULE PROTEIN (HPOGP). Black and grey boxes highlight identical and similar residues, respectively. Sequences were aligned using MultAlin and ExPASy BoxShade software. Black bars indicate peptides that were detected with high confidence in mass spectrometry measurements of L. incisa LB protein fractions. B, Hydrophobicity of amino acid positions in the sequence of LiOGP compared to HPOGP and A. thaliana OLEOSIN1. The hydrophobicity score was determined using ExPASy ProtScale software with the Kyte \& Doolittle amino acid scale and a window size of 19 residues. 

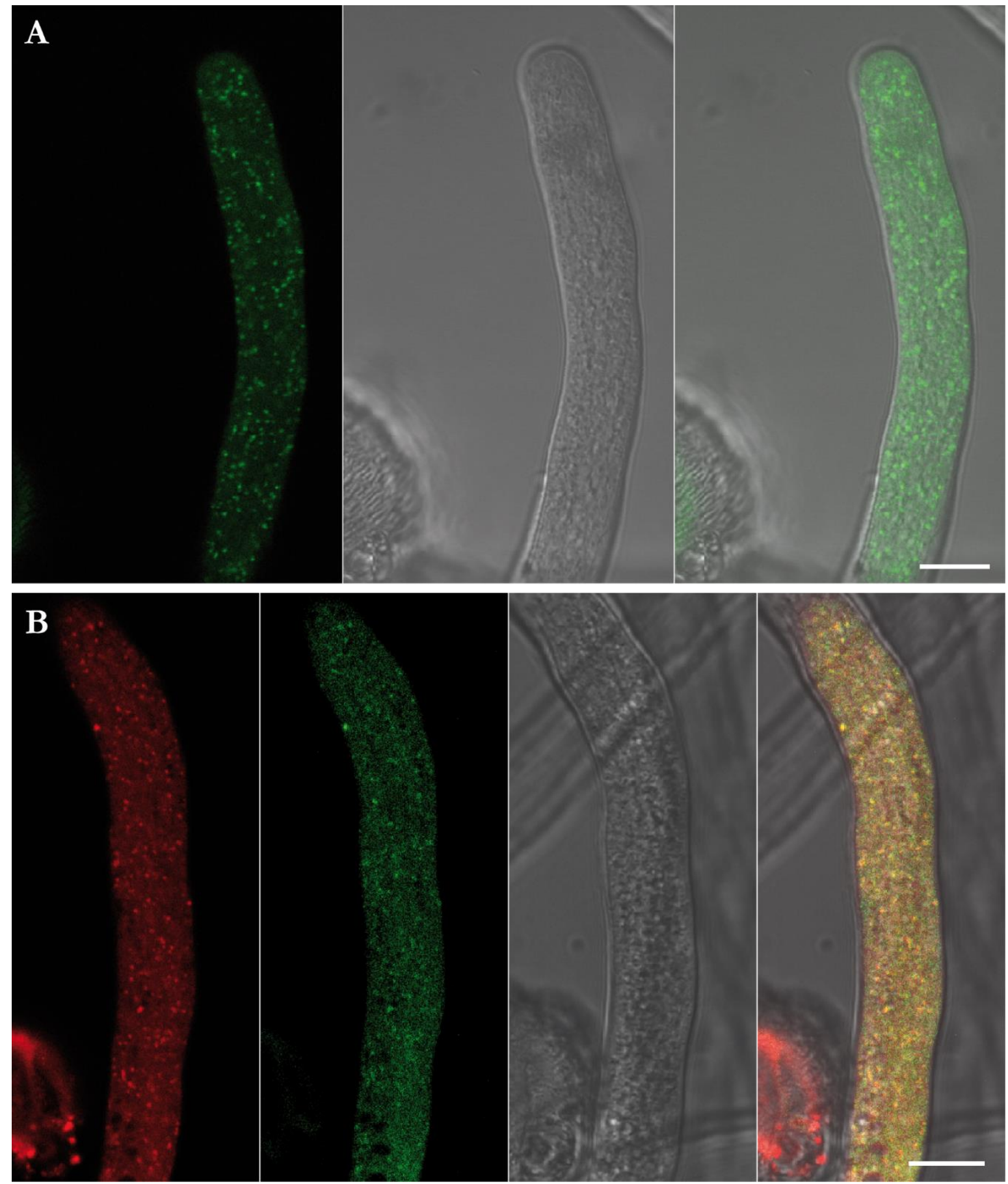

Figure 7: Subcellular localization of L. incisa OIL GLOBULE PROTEIN (LiOGP)-mVenus fusion in N. tabacum pollen tubes. Fluorescence was documented by confocal laser scanning microscopy after $6 \mathrm{~h}$ of pollen germination. Scale bars $=10 \mu \mathrm{m}$. A, Unstained pollen tube expressing the LiOGP-mVenus fusion. From left to right: mVenus fluorescence, brightfield image, merged image. 8 out of 8 pollen tubes analyzed showed comparable results. B, Pollen tube expressing the LiOGP-mVenus fusion and stained for LBs with Nile Red. From left to right: Nile Red fluorescence, mVenus fluorescence, brightfield image, merged image. 9 out of 9 pollen tubes analyzed showed comparable results. 


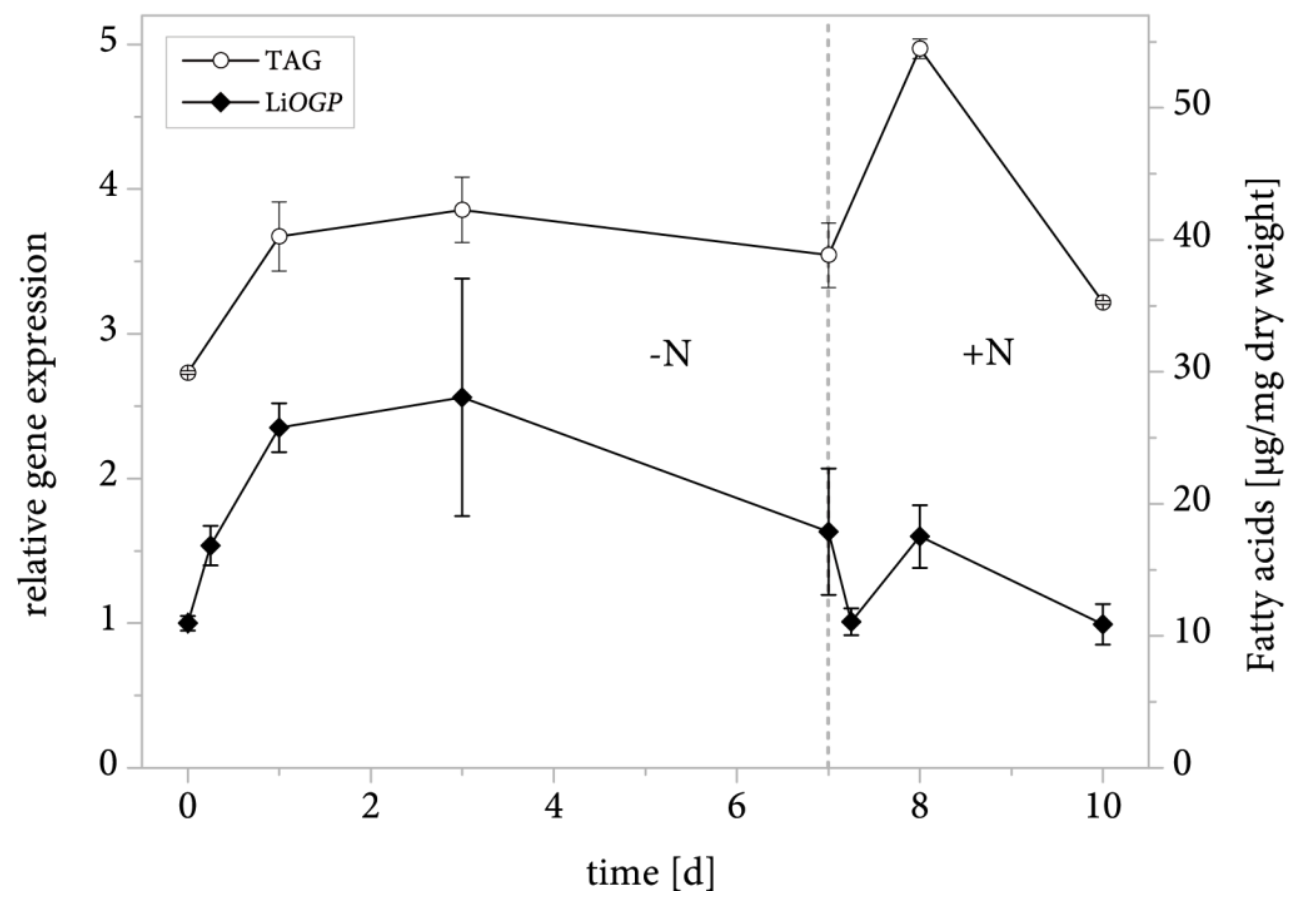

Figure 8: Expression of the L. incisa OIL GLOBULE PROTEIN (LiOGP) gene in response to changes in nitrogen supply. Transcript levels were determined by quantitative real-time PCR and normalized to RIBOSOMAL PROTEIN S21 transcripts. Expression is shown relative to time point 0 and error bars represent the standard error of the mean for three batches cultivated in parallel in a single experiment. The dotted line indicates nitrogen resupply and TAG levels are shown for comparison.

\subsubsection{Characterization of the L. incisa g15430 protein}

Protein g15430 is highly enriched in LB extracts of L. incisa and was therefore chosen for further analysis. The subcellular localization of g15430 at the surface of LBs was confirmed by heterologous expression in tobacco pollen tubes as described above (Figure 9).

Peptides covering almost $65 \%$ of the amino acid sequence contributed to the identification of the protein in the algal LB fraction (Table 31 and Figure 10A). Searches for homologous amino acid sequences or tertiary structures in other organisms yielded no results and a pronounced hydrophobic stretch of amino acids as found in AtOLEOSIN1 is absent from the sequence (Figure 10B). A conserved domain of unknown function (PFAM accession: DUF 4057) spans 70 amino acids in g15430. This domain has also been identified in uncharacterized proteins of the lycophyte Selaginella moellendorfii, the moss Physcomitrella patens, 27 species of flowering plant including $A$. thalian $a$ and other oil seed plants as well as the unicellular green algal species Micromonas pusilla, Ostreococcus tauri and Ostreococcus lucimarinus (PFAM). 


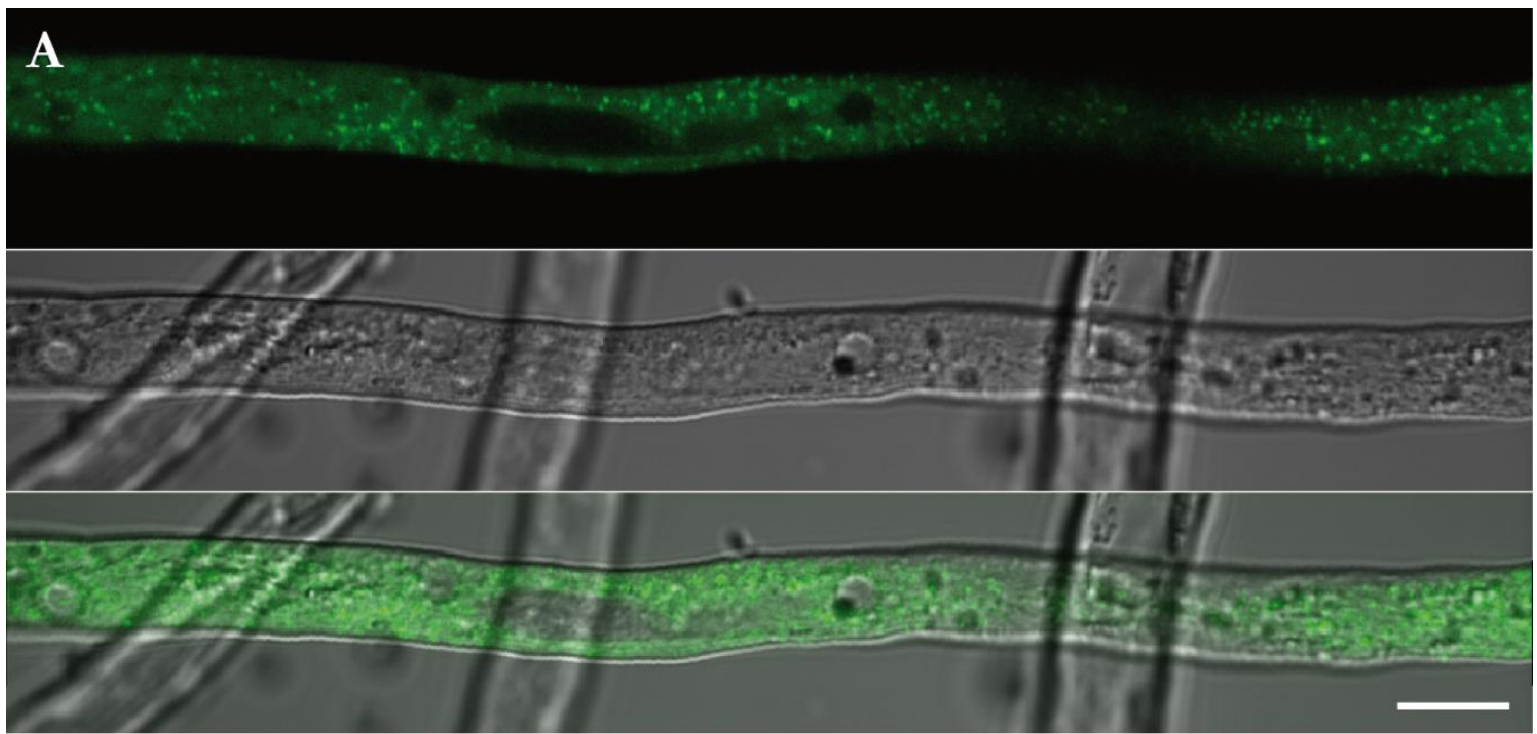

B

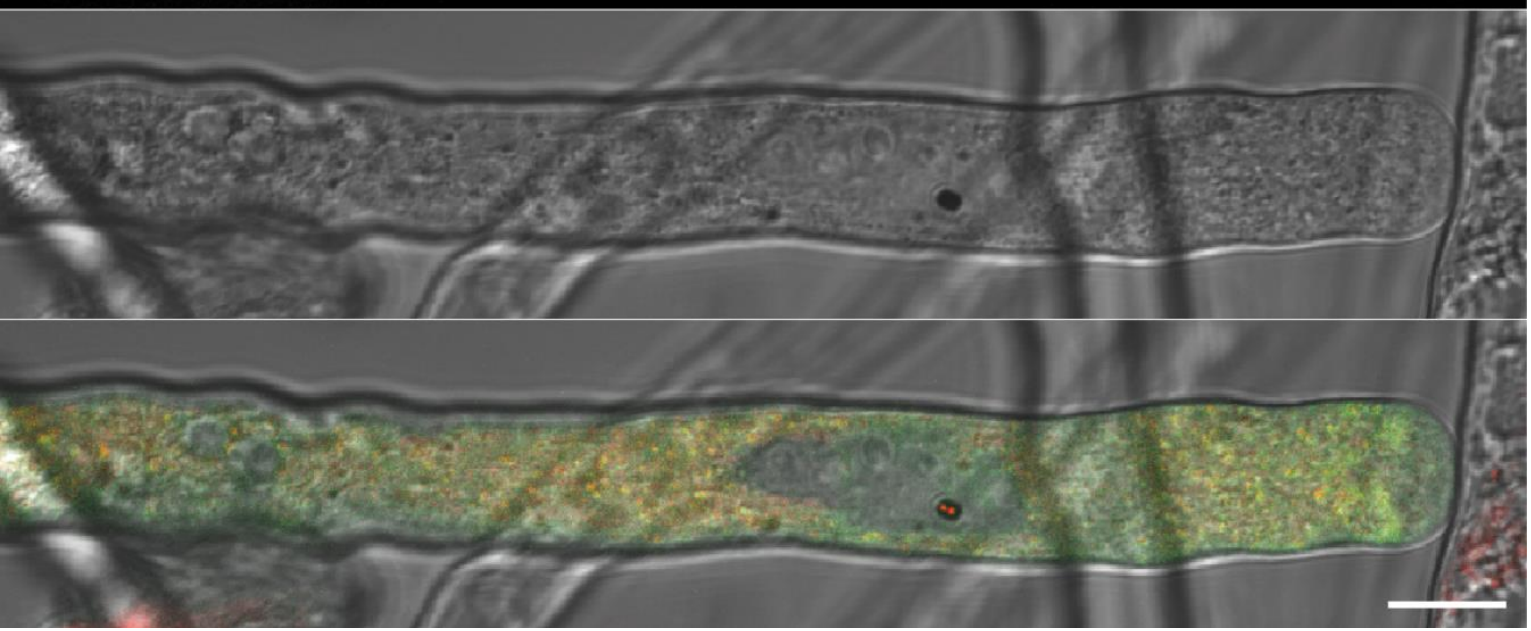

Figure 9: Subcellular localization of L. incisa g15430-mVenus fusion in N. tabacum pollen tubes. Fluorescence was documented by confocal laser scanning microscopy after $6 \mathrm{~h}$ of pollen germination. Scale bars $=10 \mu \mathrm{m}$. A, Unstained pollen tube expressing the g15430-mVenus fusion. From top to bottom: mVenus fluorescence, brightfield image, merged image. Three out of three pollen tubes analyzed showed comparable results. B, Pollen tube expressing the g15430-mVenus fusion and stained for LBs with Nile Red. From top to bottom: Nile Red fluorescence, mVenus fluorescence, brightfield image, merged image. Four out of four pollen tubes analyzed showed comparable results. 
A

g15430 1 MYNADGSMRAIR $\overline{\text { RPAHSSGGGQSSWSGGWAADAKENEPRNTVGSNSGNVAGGPSPAAAGM }}$

61 K̄SKSAGAEAGKGSRQPADAKIK $\overline{\text { ALQGSNIFGNHSDAATVQDTTAKLQQVQVQDMPAAGSA }}$

$121 \overline{\text { TIANSGESGVRDISDAKRRALYQTNVFTHTDEPANWRSAEA IASKPEVPAVPAGSAGQFR }}$

180 AAEPGARDISDAKKKS LYQTR $\overline{V F G T A D A A S P A A Q Q V S S L K H K E M S G H N I F G A A E E ~ P A P R A ~}$

240 AGQSGR RPPGGASTFSFGNSGAESAAAPSQGGSR $Q T A G G S S T F S F G W$

$\mathrm{B}$

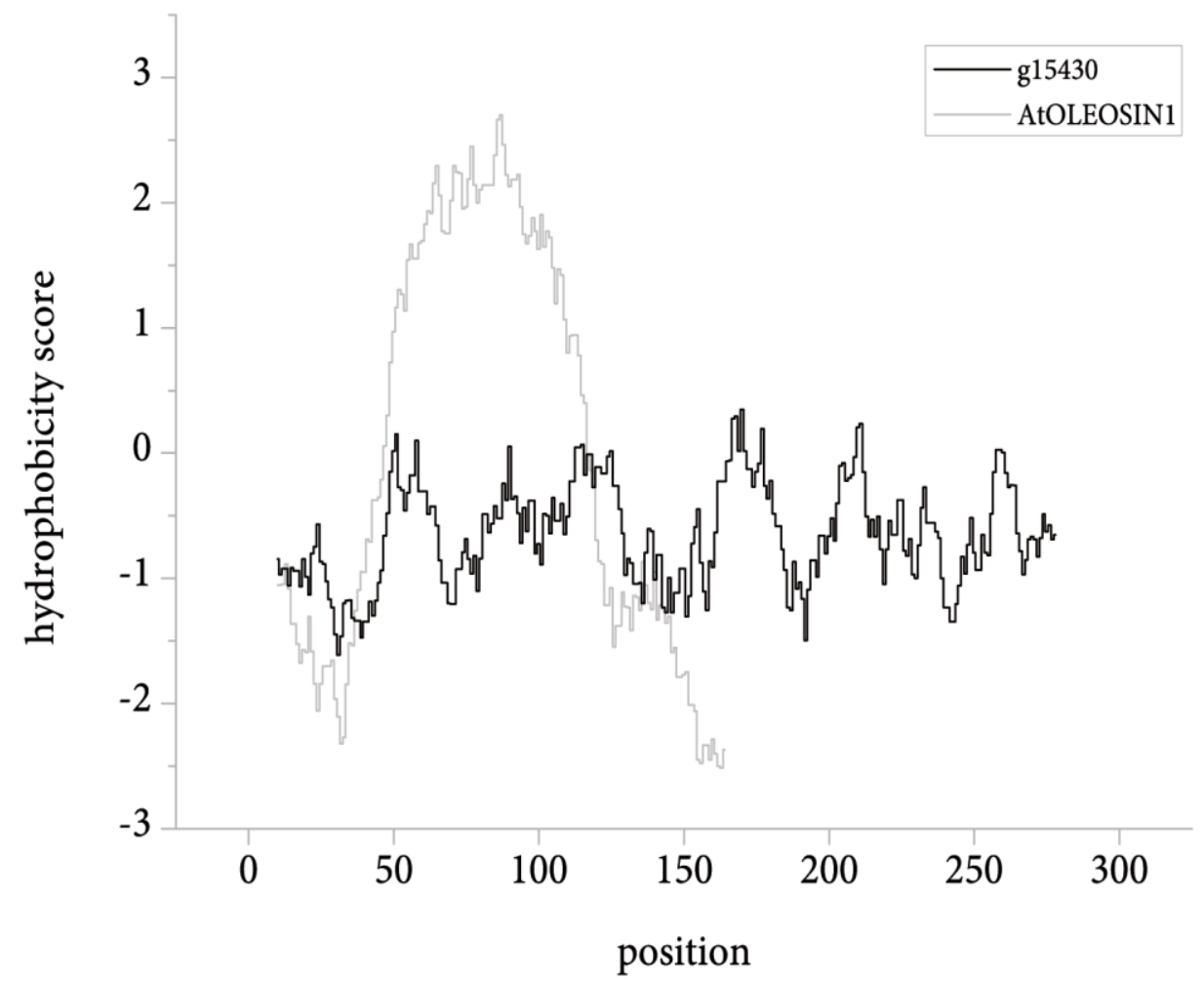

Figure 10: Features of the L. incis a g15430 amino acid sequence. A, The amino acid sequence with black bars indicating peptides that were detected with high confidence in mass spectrometry measurements of $L$. incisa LB protein fractions. The grey bar represents the domain of unknown function (DUF 4057) detected using the PFAM server. B, Hydrophobicity of amino acid positions in the sequence of g15430 compared to A. thaliana OLEOSIN1. The hydrophobicity score was determined using EXPASy ProtScale software with the Kyte \& Doolittle amino acid scale and a window size of 19 residues. 


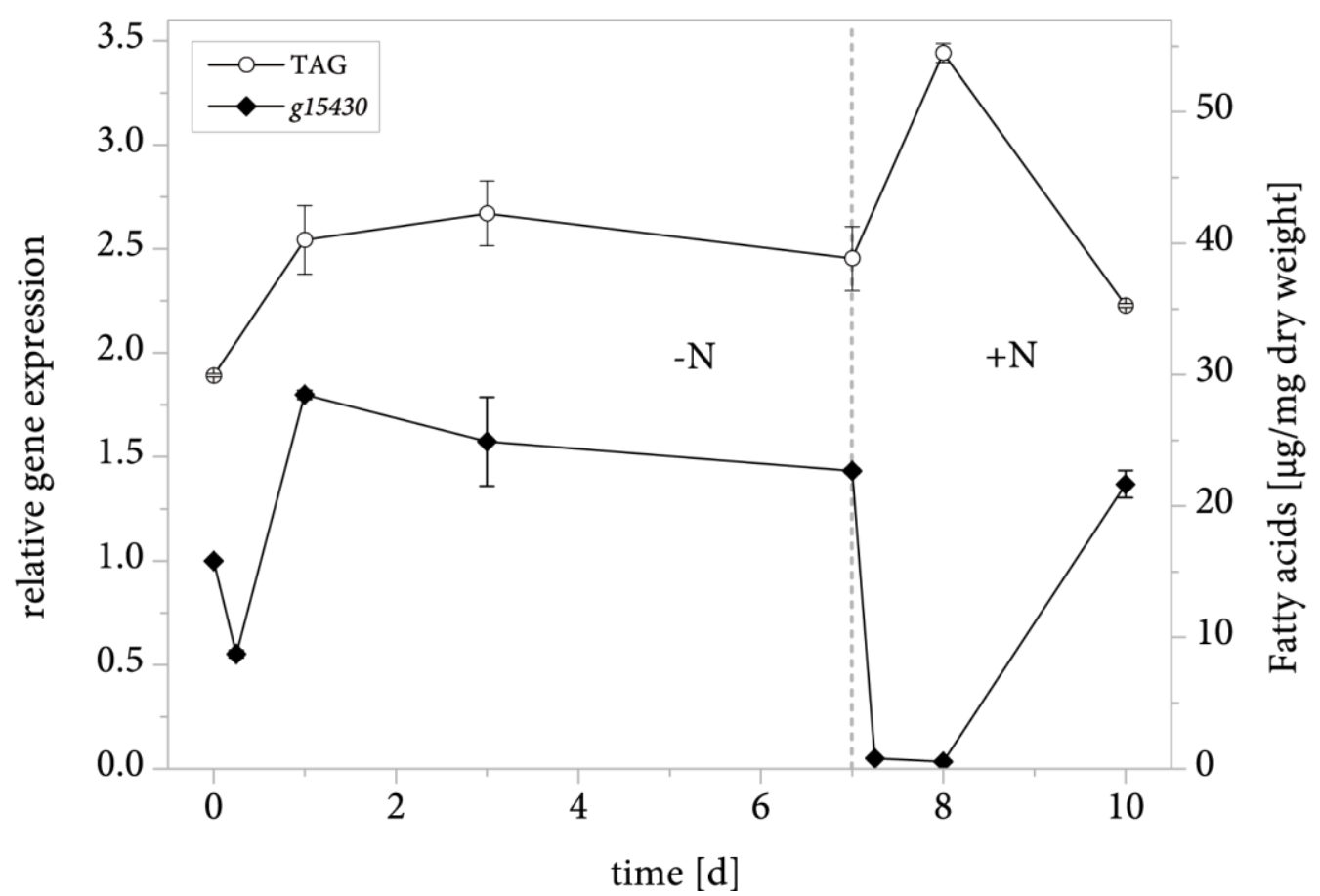

Figure 11: Expression of the L. incisa g15430 gene in response to changes in nitrogen supply. Transcript levels were determined by quantitative real-time PCR and normalized to RIBOSOMAL PROTEIN S21 transcripts. Expression is shown relative to time point $O$ and error bars represent the standard error of the mean for three batches cultivated in parallel in a single experiment. The dotted line indicates nitrogen resupply and TAG levels are shown for comparison.

The g15430 gene undergoes a brief drop in expression during the first hours of cultivation without nitrogen before exhibiting a sharp increase at day 1 (Figure 11). From this point on, the level of transcripts mirrors that of TAG, remaining almost constant until nitrogen is resupplied, which is associated with a rapid reduction in g15430 gene expression. At $3 \mathrm{~d}$ of growth in full media, the transcripts once again reach their former plateau level as TAG is degraded. 


\subsubsection{Characterization of the L. incisa g13747 protein}

Protein g13747 was identified as a putatively LB associated protein as it is strongly enriched in the L. incisa LB fraction and a region of increased hydrophobicity was detected in a preliminary analysis. The tryptic peptides used to identify the protein in mass spectrometry cover $37 \%$ of the amino acid sequence (Table 31).

When heterologously expressed in the pollen tubes of N. tabacum, the g13747 protein exhibits the same distinct punctate pattern as for other LB localized proteins (

Figure 12). However, transformation efficiency was not sufficient to permit a colocalization assay with the neutral lipid specific dye Nile Red.

The primary structure of the protein allows little speculation as to the protein's function as the amino acid sequence is not similar to any other known proteins or conserved domains, however the $\mathrm{N}$-terminus contains a marked hydrophobic section comparable to that found in AtOLEOSIN1 (Figure 13B).

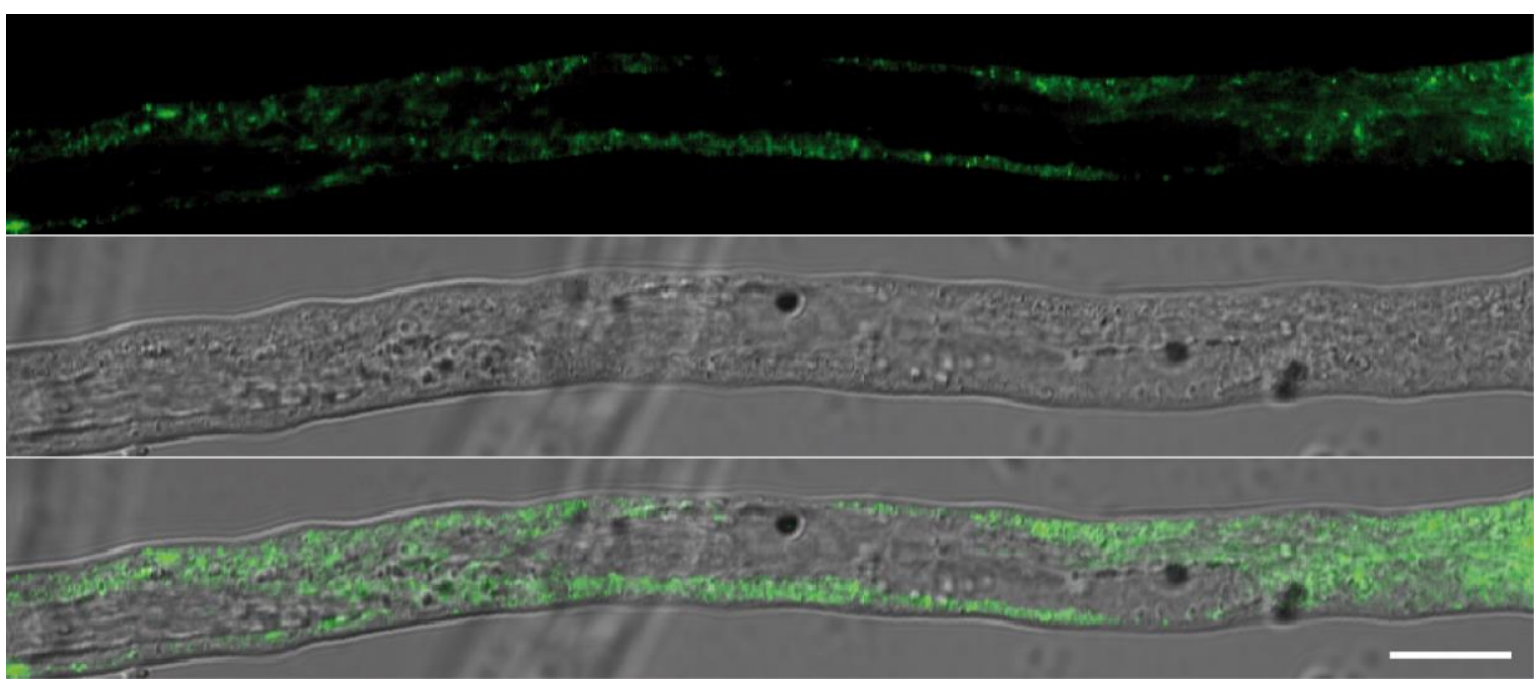

Figure 12: Subcellular localization of mVenus-L. incisa g13747 fusion in N. tabacum pollen tubes. Fluorescence was documented by confocal laser scanning microscopy after $6 \mathrm{~h}$ of pollen germination. From top to bottom: mVenus fluorescence, brightfield image, merged image. A pollen tube expressing the C-terminal fusion showed comparable localization. Scale bar $=10 \mu \mathrm{m}$. 
A

g13747 1 MTKSTSSRSARDVAFSVFEVTTAVMYGVYENTLAGVVPYFVPDRTREALFQLFCELRTNS

61 PKDKRFLKKHLAAFVPETVTGWIAIASSALYATSVVAGS LFMLAGLASAALFSCVGIVLV

121 TGSFAGVALAFLALGLLCAACITGVMGTATAFGYLSALTGSAAWQFVSQHVFGGASLVPD

181 SIRDLPISRHAGKIFGSTASAHKQSDTKRASAR $\overline{A S A Q Q P P S A P Q P P K L Q P L S Q Q Q A F G S M ~}$

241 QTQDEAQCTGKQPTAGQAPSEPQQAKHAKQPAPAELPQPVSSVASVK $\overline{A P D G P T T P A P S D T}$

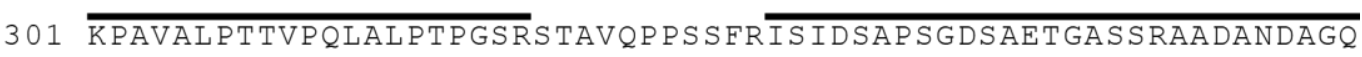

361 DAQPETDAQHAQHVKQPAPAELAKPPTTEPTAEVVSEQPKAAPKVAFADPQQAITASSAS

421 AEQLYQIKPSAVDQQPVTARHGTAATEAQQAK $\overline{\text { HAQQPAPAEPGKPPRSETPAAAAAPAAQ }}$

$481 \overline{\text { QTQRSVPEPIDVHIIGDADAAQAGP PPGAAPATR SNASK SQELFADLADTAVVEAIDDAP }}$

541 HEIDASVALNSALKTGTAQAAVVEGPAPAIQASASATHSDDTGFLTGAKGVAPNYLSTPD

601 SNSKLPAGLQTSLASIDTQPAPFSFVVPEPATAAPHTSSAAVLTGLRSADVAPQGPHLA

661 AHADQPAAPIFGHSAAQQPQQADTQAPASAMGFSAPSVHDNILFDLPRAGSNTPMLSDTK

721 PPVALSALPQPTAAIPDATTTTVQPVTSFRISIDSGDSAHTGASDPLVRSESEGSEVSAS

781 GRPKRMSSKNRR $\overline{\text { KAQAAALAASAEQ }}$

B

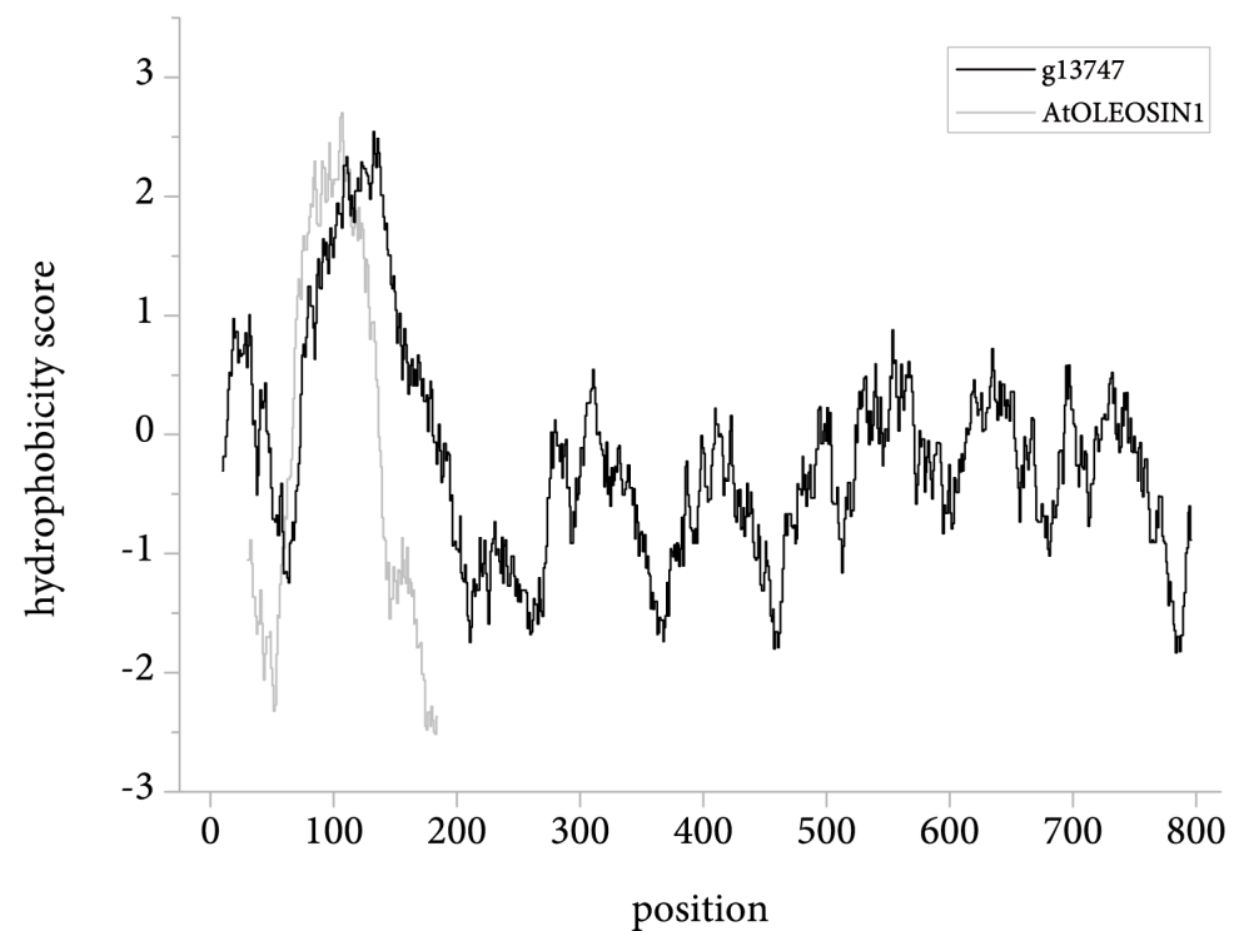

Figure 13: Features of the L. incisa g13747 amino acid sequence. A, Black bars indicate peptides that were detected with high confidence in mass spectrometry measurements of L. incisa LB protein fractions. B, Hydrophobicity of amino acid positions in the sequence of L. incisa g13747 compared to A. thaliana OLEOSIN1. The hydrophobicity score was determined using ExPASy ProtScale software with the Kyte \& Doolittle amino acid scale and a window size of 19 residues. 


\subsubsection{Characterization of the L. incisa g13945 protein}

LB localization of g13945 could be confirmed by heterologous expression in tobacco pollen tubes and colocalization with Nile Red (Figure 14).

The amino acid sequence, $49 \%$ of which were identified in the initial mass spectrometric analysis (Table 31), is characterized by a partial conserved lipase class 3 domain (PFAM accession PF01764, Figure 15). This domain is found in many lipases and contains the characteristic catalytic triad of serine, aspartate and histidine (Derewenda and Derewenda, 1991). The serine residue is part of a nucleophilic elbow that consists of a highly conserved glycine-X-serine-X-glycine motif, with $\mathrm{X}$ representing any residue. This motif as well as the catalytic aspartate can be identified in the conserved domain in the g13945 amino acid sequence, whereas the C-terminal part of the protein lacks homology to any other known protein in both amino acid sequence and predicted structure (data not shown), so that the identity and location of the third catalytic residue cannot be predicted.

Overall the g13945 protein does not exhibit a striking sequence similarity with any single known protein, however its predicted secondary structure closely matches that of a group of fungal and yeast secretory lipases, as determined by Phyre2 search. A representative of this group, Malassezia globosa LIPASE1 (MgLIP1) is included in Figure 15. These lipases are known to degrade mono-, di- and triacylglycerol, however MgLIP1 differs from its structural homologs in strictly not accepting TAG as a substrate (DeAngelis et al., 2007). The crystal structure of this protein has been published (Xu et al., 2012) (PDB accession 3UUE) and the authors of the study suggest the presence of the relatively large tryptophane residue directly adjacent to the catalytic aspartate to be a determinant in this unusual substrate specificity. All other lipases that are highly similar in structure and do hydrolyze TAG have a smaller residue in this position, which is also the case for L. incisa g13945 (see Figures 15 and 17).

In L. incisa cells exposed to nitrogen limitation, g13945 transcript levels rapidly increase more than 10-fold during the first $3 \mathrm{~d}$ and undergo a sharp drop following nitrogen resupply, reaching the initial range within only $1 \mathrm{~d}$ (Figure 16).

Protein g13945 was tested for TAG lipase activity by complementation assays in $A$. thaliana. The A. thaliana sdp1/sdp1-L mutant lacks the lipases responsible for the bulk of TAG degradation in germinating seeds: SUGAR-DEPENDENT1 (SDP1) and SDP1-LIKE (SDP1-L) (Kelly et al., 2011). Etiolated mutant seedlings have been shown to be limited in seedling growth in comparison with etiolated wildtype seedlings, a phenotype that can be 


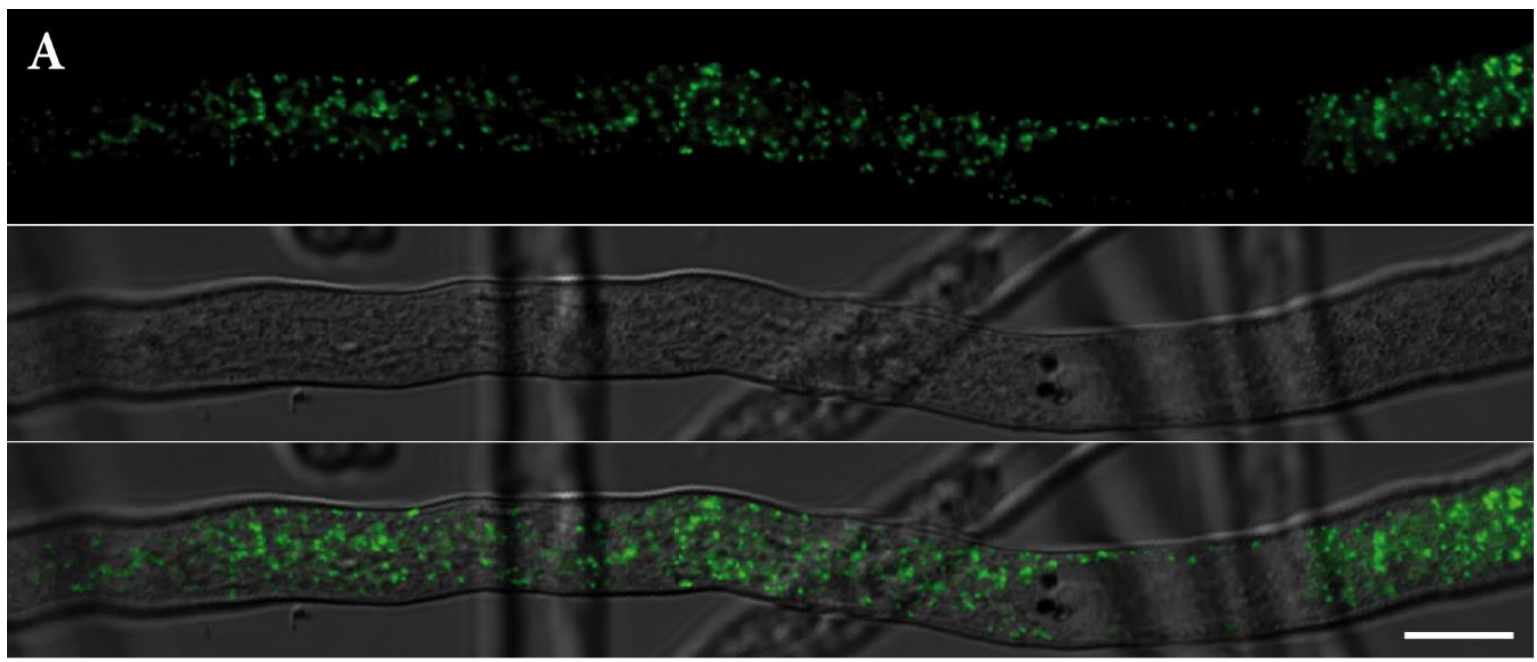

B
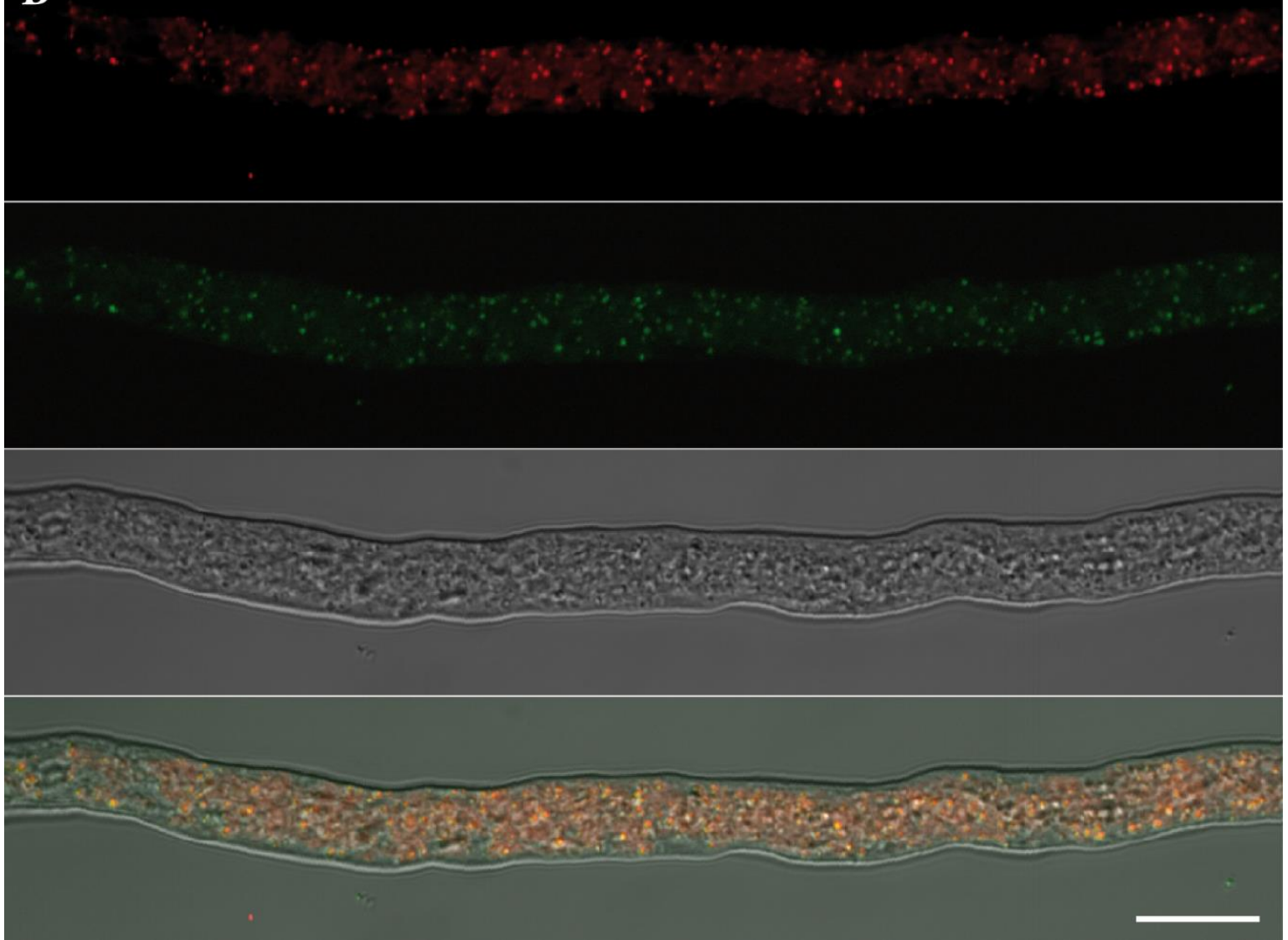

Figure 14: Subcellular localization of L. incisa g13945-mVenus fusion in N. tabacum pollen tubes. Fluorescence was documented by confocal laser scanning microscopy after $6 \mathrm{~h}$ of pollen germination. Scale bars $=10 \mu \mathrm{m}$. A, Unstained pollen tube expressing the g13945-mVenus fusion. From top to bottom: mVenus fluorescence, brightfield image, merged image. 7 out of 7 pollen tubes analyzed showed comparable results. B, Pollen tube expressing the g13945-mVenus fusion and stained for LBs with Nile Red. From top to bottom: Nile Red fluorescence, mVenus fluorescence, brightfield image, merged image. Three out of three pollen tubes analyzed showed comparable results. 
rescued by supplying an external carbon source such as sucrose. The algal g13945 gene was placed under the control of the seed specific Brassica napus napin A (napA) promoter and expressed in the mutant background. Seeds were germinated in the dark for $5 \mathrm{~d}$ on solid media either without an added carbon source or supplemented with sucrose. As depicted in Figure $18 \mathrm{~A}$, a minor but significant increase in the ratio of hypocotyl length between the treatments can be observed in the complemented lines in comparison with the double knockout mutant. Hypocotyl length after germination in the two different conditions is shown separately in Supplemental figure $2 \mathrm{~A}$.

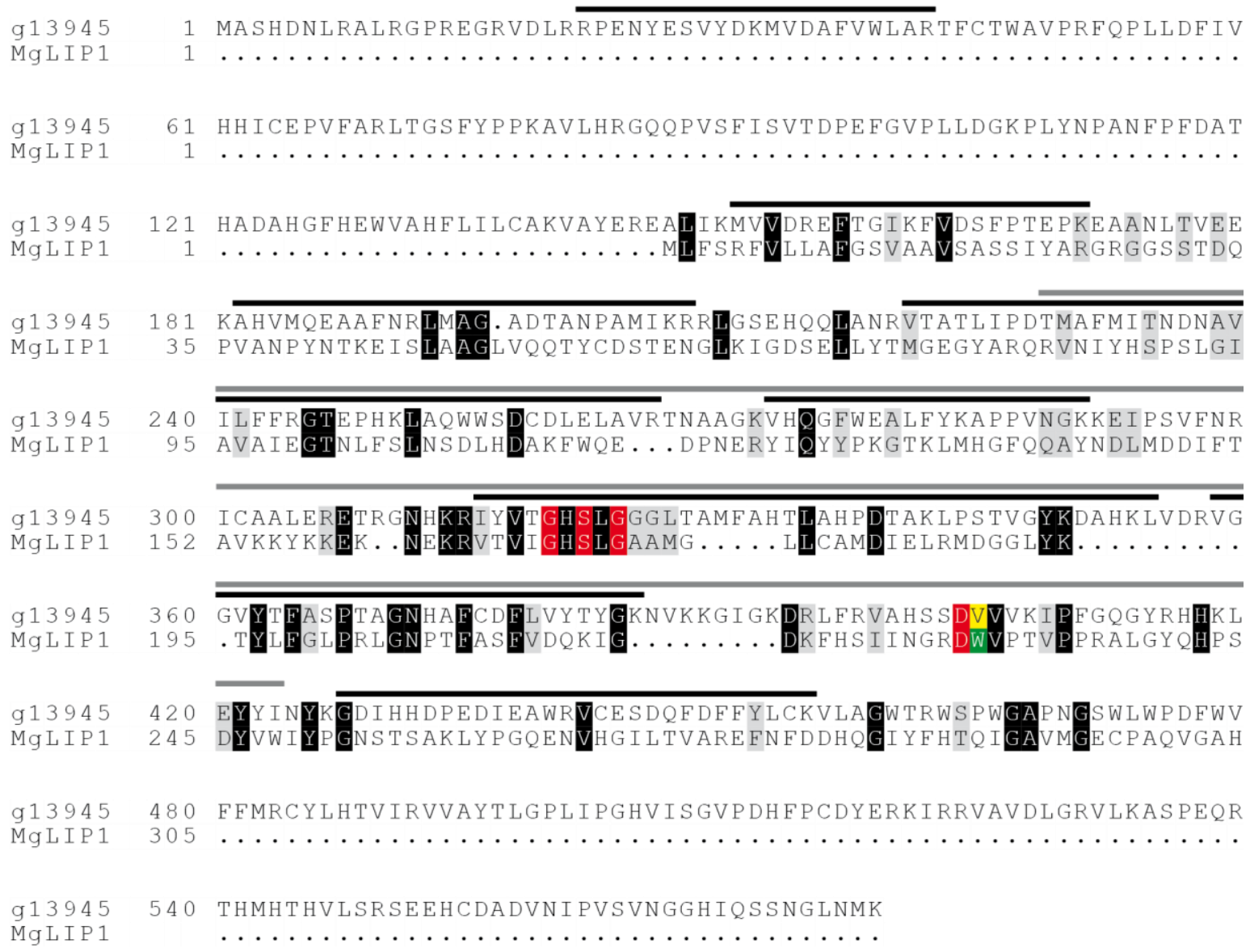

Figure 15: Features of the L. incisa g13945 amino acid sequence. Similarities with the amino acid sequence of M. globosa LIPASE 1 (MgLIP1) are shown with black and grey boxes highlighting identical and similar residues, respectively. Sequences were aligned using MultAlin and ExPASy BoxShade software. Components of the active site as determined by structural homology are marked in red and the residues of different sizes adjacent to the catalytic aspartate are highlighted in yellow and green for the algal and the fungal protein, respectively. The grey bar represents the conserved lipase class 3 domain. Black bars indicate peptides that were detected with high confidence in mass spectrometry measurements of L. incisa LB protein fractions. 


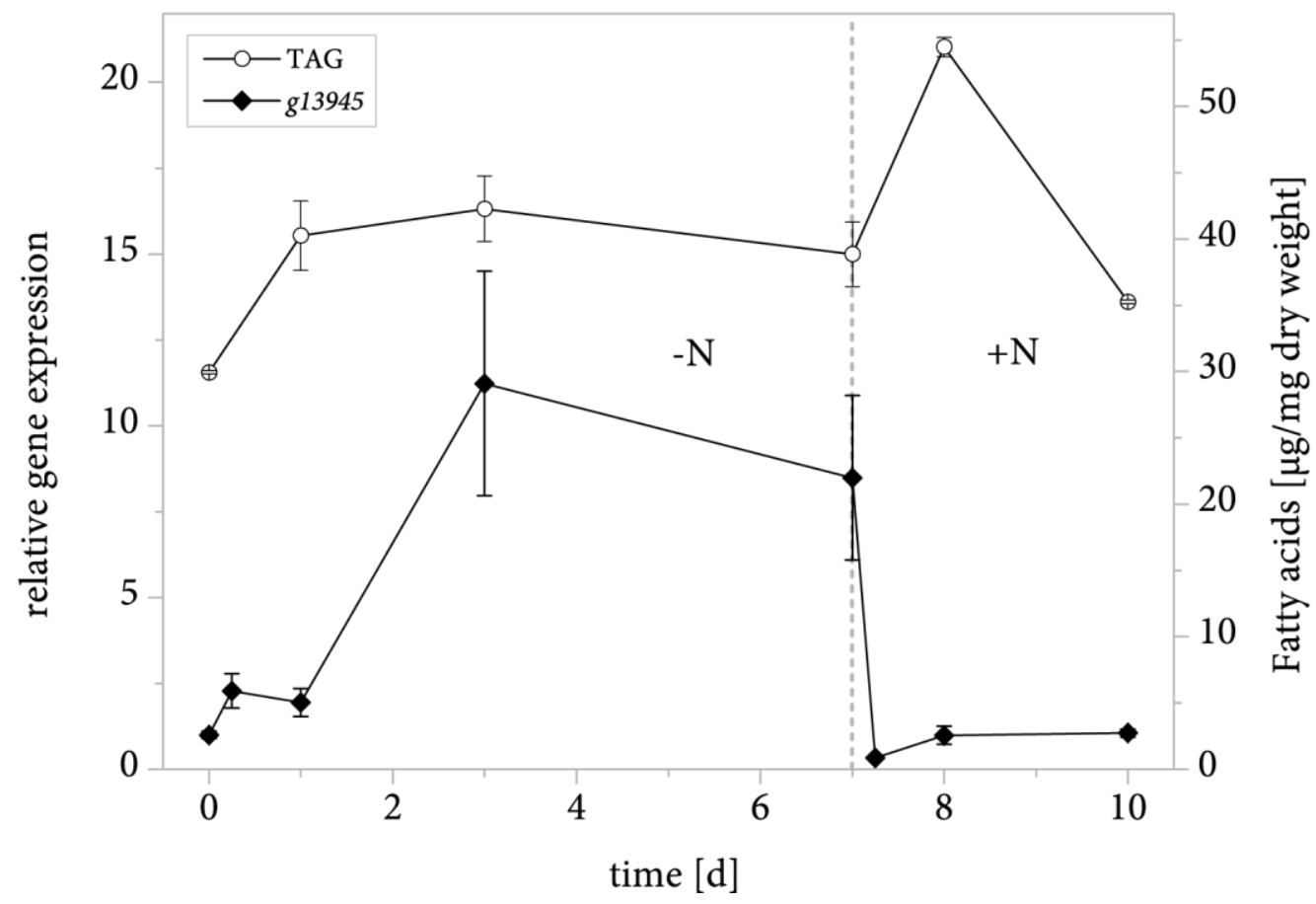

Figure 17: Expression of the L. incisa g13945 gene in response to changes in nitrogen supply. Transcript levels were determined by quantitative real-time PCR and normalized to RIBOSOMAL PROTEIN S21 transcripts. Expression is shown relative to time point $O$ and error bars represent the standard error of the mean for three batches cultivated in parallel in a single experiment. The dotted line indicates nitrogen resupply and TAG levels are shown for comparison.
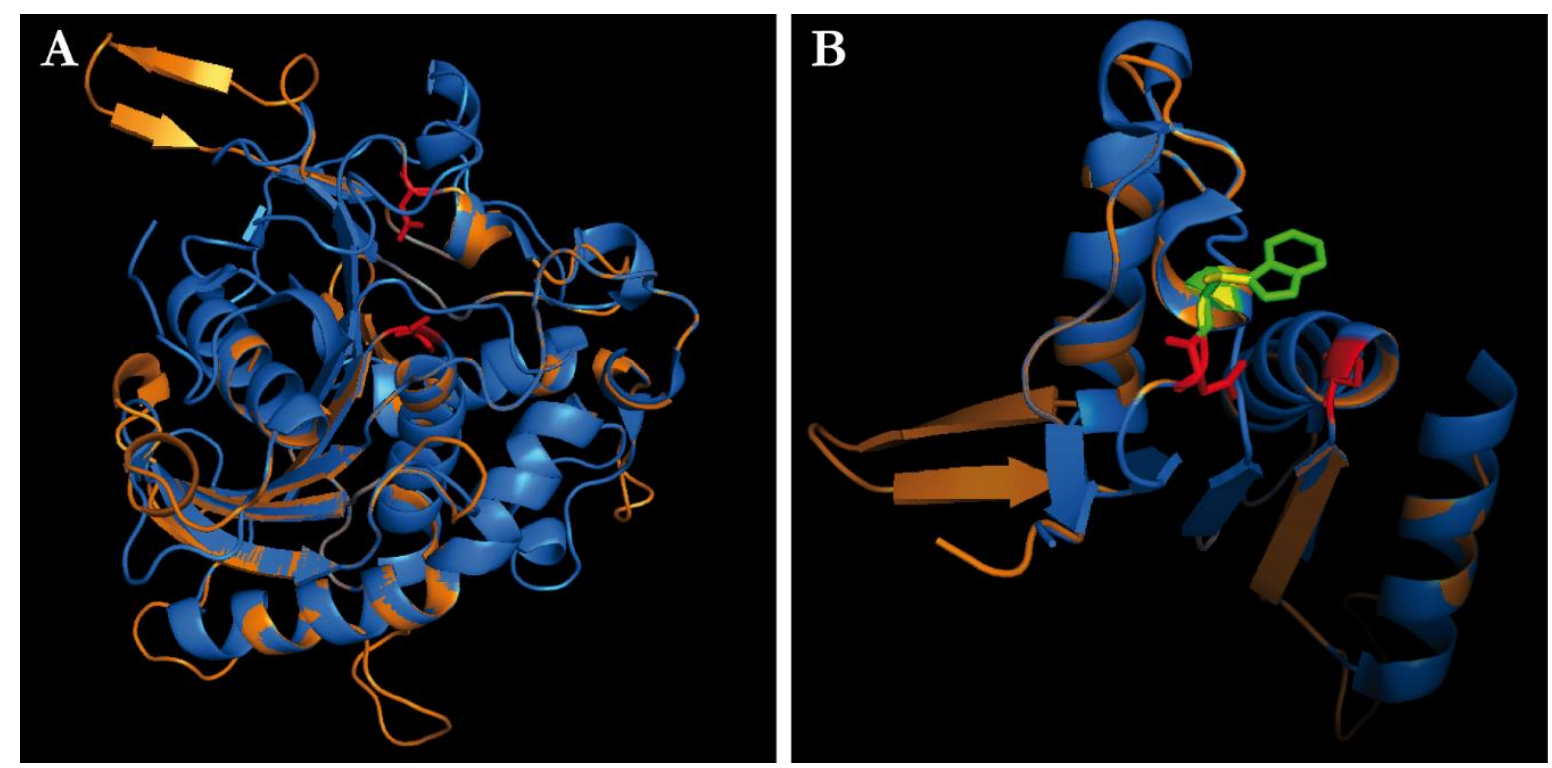

Figure 16: Predicted structural homology of L. incisa g13945 and M. globosa LIPASE 1 (MgLIP1). The amino acid sequence of g13945 from residue 190 - 447 (shown in orange) was modeled to the crystal structure of MgLIP1 (shown in blue) using the Phyre 2 server and aligned to it. A, The entire alignment is shown with the catalytic serine and aspartate residues in both proteins depicted as red sticks. $B$, Magnification of the active site with the catalytic serine and aspartate residues shown in red. In addition, the tryptophane adjacent to the catalytic aspartate in the fungal lipase is depicted in green and the valine that occupies this position in the algal protein is shown in yellow. 
The capacity of L. incisa g13945 to hydrolyze TAG was additionally tested by supplementing crude cell extracts of $3 \mathrm{~d}$ old seedlings with radioactive triolein and monitoring its degradation. The fatty acid moieties of the molecule are labeled with the ${ }^{14} \mathrm{C}$ isotope, permitting highly sensitive detection of TAG and free fatty acids in thin layer chromatography (TLC). Seedlings were allowed to germinate for $3 \mathrm{~d}$ before analysis, a developmental stage characterized by TAG degradation in the wildtype but not the $s d p 1 / s d p 1-L$ mutant (Kelly et al., 2011). Crude extracts of the seedlings were incubated with the labeled substrate in buffers at $\mathrm{pH} 6,7$ and 8, accounting

A

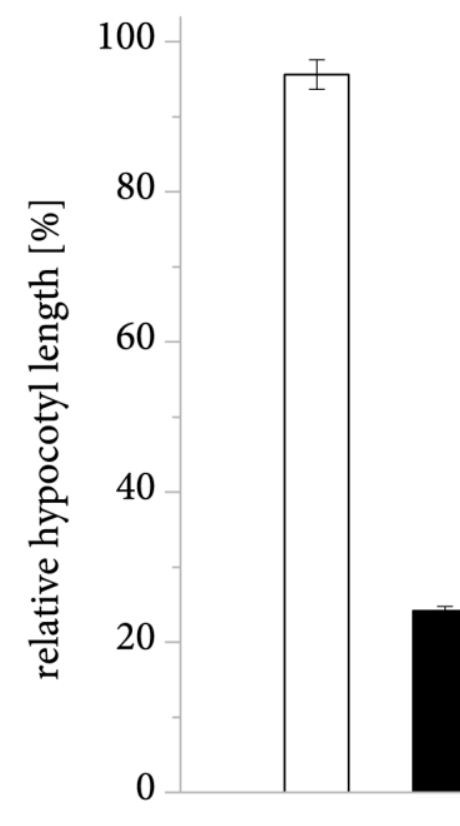

B

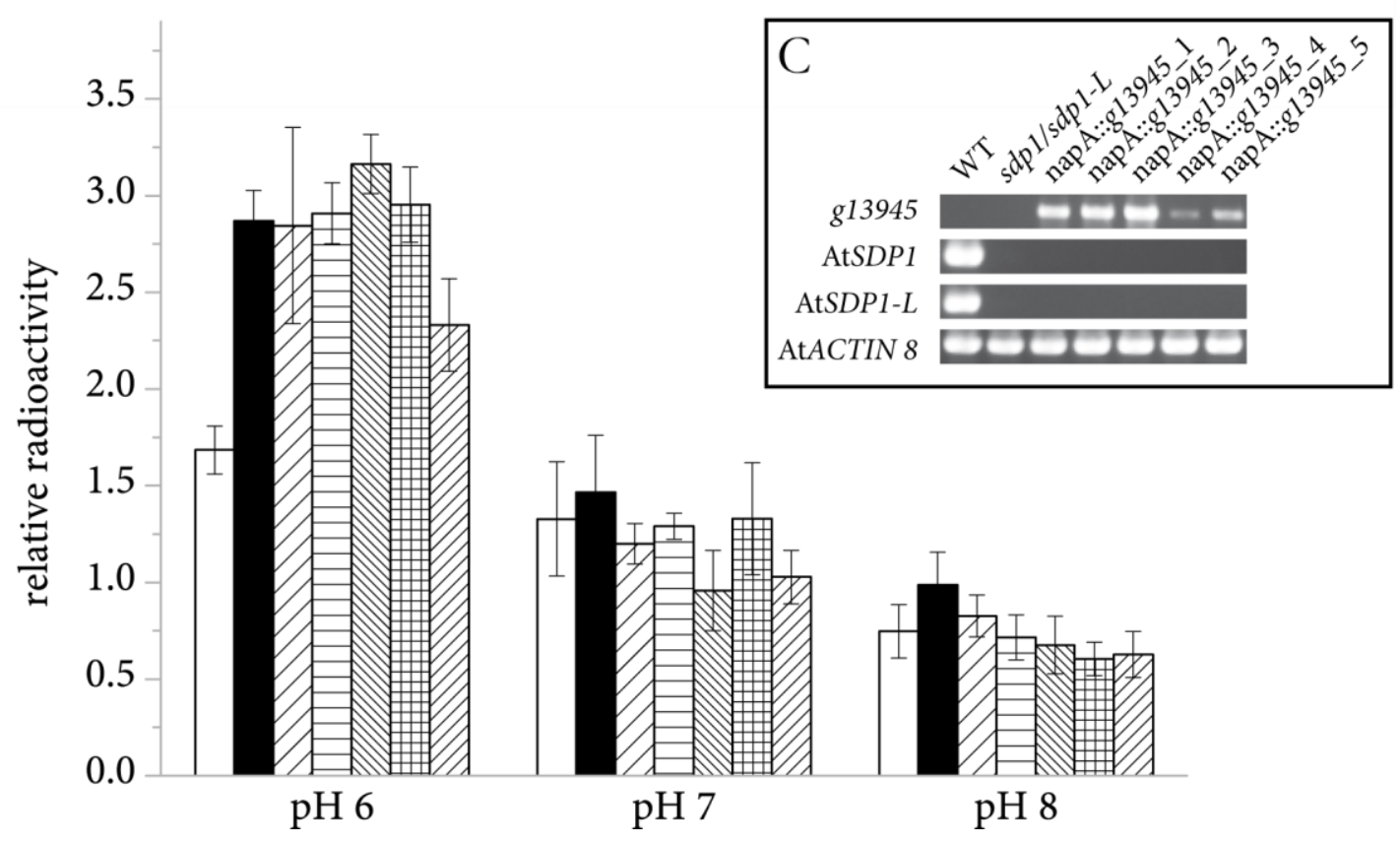


Figure 18: Lipase activity assays with L. incisa g13945 in A. thaliana. Sdp1/sdp1-L seedlings with the Brassica napus napinA (napA) promoter controlling expression of the L. incisa g13945 gene in five independent lines are compared to the mutant carrying an empty vector as well as the wildtype (WT). A, Hypocotyl length of seedlings after 5 d of germination in the dark on $1 / 2$ MS agar plates without sucrose relative to growth with sucrose. For each growth condition and independent line, three or four batches of at least 15 seedlings were measured in a single experiment and hypocotyl length of seedlings germinated without sucrose was divided by the average value for the same line with sucrose. Error bars represent the standard error of the mean. Asterisks denote measurements on transgenic lines deviating significantly from the mutant (two-sided Student's T-test, $\alpha=0.05$ ). B, Degradation of ${ }^{14} C$ labeled TAG in crude extracts of $3 \mathrm{~d}$ old seedlings. Following incubation in different buffers, the hexane extract of each reaction was separated by thin layer chromatography (TLC) and radioactivity was detected across the TLC plate. Relative radioactivity was calculated as the ratio of radiation emitted by TAG and free fatty acids as measured densitometrically. Error bars represent the standard deviation of three technical replicates in a single experiment. C, Confirmation of gene expression in dry seeds of independent lines.

for the range of physiological conditions in different cellular compartments, in three technical replicates each. Lipids were extracted from the reactions, separated by TLC (see Supplemental figure 3 for an example) and the ratio of residual radioactivity originating from the TAG and free fatty acid bands was calculated (Figure 18C), lower values thus reflecting a higher TAG turnover. The presence of lipid synthesizing enzymes in the cell extract only had a minor effect on free fatty acid and TAG amounts as demonstrated by supplementing $s d p 1 /$ sdp $1-L$ seedling extracts with radioactively labeled 16:0 (see Supplemental figure 3). Due to high background lipase activity in the crude cell extracts, a significant difference between TAG degradation in wildtype seedlings and the double knockout mutant could not be observed for reactions at $\mathrm{pH}$ 7 or 8 and the values for the complemented lines are in the same range of the controls. At pH 6 however, the mutant as well as the complemented lines are markedly reduced in their ability to hydrolyze TAG by comparison with the wildtype.

Gene expression in all lines used for the lipase activity assays was confirmed in dry seeds as shown in Figure 18B. 


\subsection{Identification of enzymes involved in triacylglycerol degradation}

Further candidate genes possibly involved in the breakdown of the main storage lipid in L. incisa were sought in the genome sequenced by the GIAVAP consortium (unpublished data). 45 predicted genes were categorized as putative lipases based on a search using the InterProScan software tool (data not shown). The list of candidates was searched for homologs of known TAG lipases and two of these were selected for further analysis.

\subsubsection{Characterization of the putative lipase g2905}

The g2905 protein was identified as a putative TAG lipase based on sequence homology with human GASTRIC LIPASE A (HsLIPA) which has TAG hydrolyzing activity (Bodmer et al., 1987) (Figure 19). The protein contains an $\alpha / \beta$ fold domain characteristic of a broad range of hydrolases (Ollis et al., 1992) which is preceded in the amino acid sequence by a domain of unknown function frequently found in this location in lipases (PFAM). A signal peptide spanning the first 20 amino acids was detected and predicted to target the protein to the ER using TargetP software. Based on the crystal structure of the human enzyme (PDB accession 1HLG, Robinson and Schlösser, 1978) and the sequence alignment shown in Figure 19, the catalytic amino acid residues of g2905 could be identified as S192 (as part of the conserved

glycine-X-serine-X-glycine motif), D373 and H404. An additional algal homolog was found in the genome of $C$. reinhardtii (CrLIPG2). The expression of this gene is known to be induced in response to nitrogen limitation (Boyle et al., 2012) and downregulated in heat stressed cells (Légeret et al., 2016).

g2905 gene expression was analyzed in L. incis a cultivated under nitrogen limited and replete conditions (

Figure 20). Transcript levels undergo a minor increase during $7 \mathrm{~d}$ of nitrogen starvation and increase approximately 2 -fold in parallel to the TAG amount when nitrogen is resupplied.

The g2905 protein was tested for TAG lipase activity by complementation of the $A$. thaliana $s d p 1 / s d p 1-L$ mutant as described above. In these assays, minor enhancements of hypocotyl growth in etiolated seedlings could be observed in four out of five complemented lines in comparison with the mutant (Figure 22A, Supplemental figure 2B). Hydrolysis of ${ }^{14} \mathrm{C}$ labeled TAG was comparable to both mutant and wildtype for reactions at $\mathrm{pH} 6,7$ and 8 (Figure 22B). 
Gene expression in all lines used for the lipase activity assays was confirmed in dry seeds as shown in Figure 22C.

Subcellular localization can help to elucidate possible functions of a protein, therefore the ability of g2905 to associate with LBs was investigated in the tobacco pollen tube system. The resulting pattern of fluorescence emitted by the mVenus tag (Figure 21) has little resemblance with LB localization as confirmed by the overlapping Nile Red signal (Figure 7,Figure 9,Figure 12). g2905 was not detected in any sample in the mass spectrometric analysis of nitrogen starved L. incisa cells.

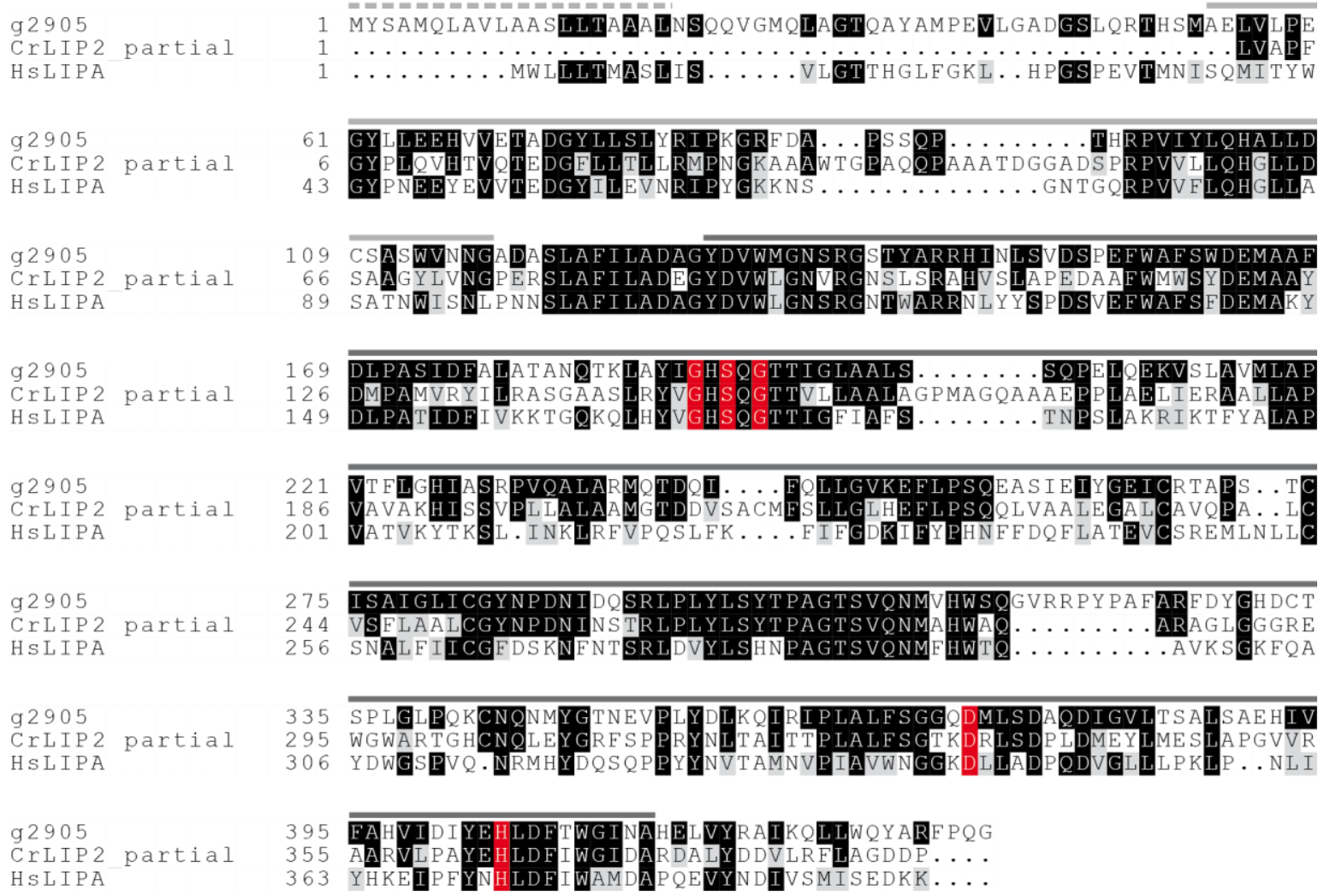

Figure 19: Features of the L. incisa g2905 amino acid sequence. Similarities with the amino acid sequences of Homo sapiens GASTRIC LIPASE A (HSLIPA) and the closest algal homolog, C. rein ha rdtii putative TRIACYLGLYCEROL LIPASE 2 (CrLIP2), are shown with black and grey boxes highlighting identical and similar residues, respectively. Sequences were aligned using MultAlin and ExPASy BoxShade software. The GXSXG motif conserved in many serine hydrolases as well as the catalytic aspartate and histidine residues are marked in red. The dotted line indicates the signal peptide as predicted using TargetP software. The dark grey bar represents the conserved $\alpha / \beta$ hydrolase domain. The light grey bar represents a domain that is conserved at the $N$-terminus of the $\alpha / \beta$ domain in many lipases. 


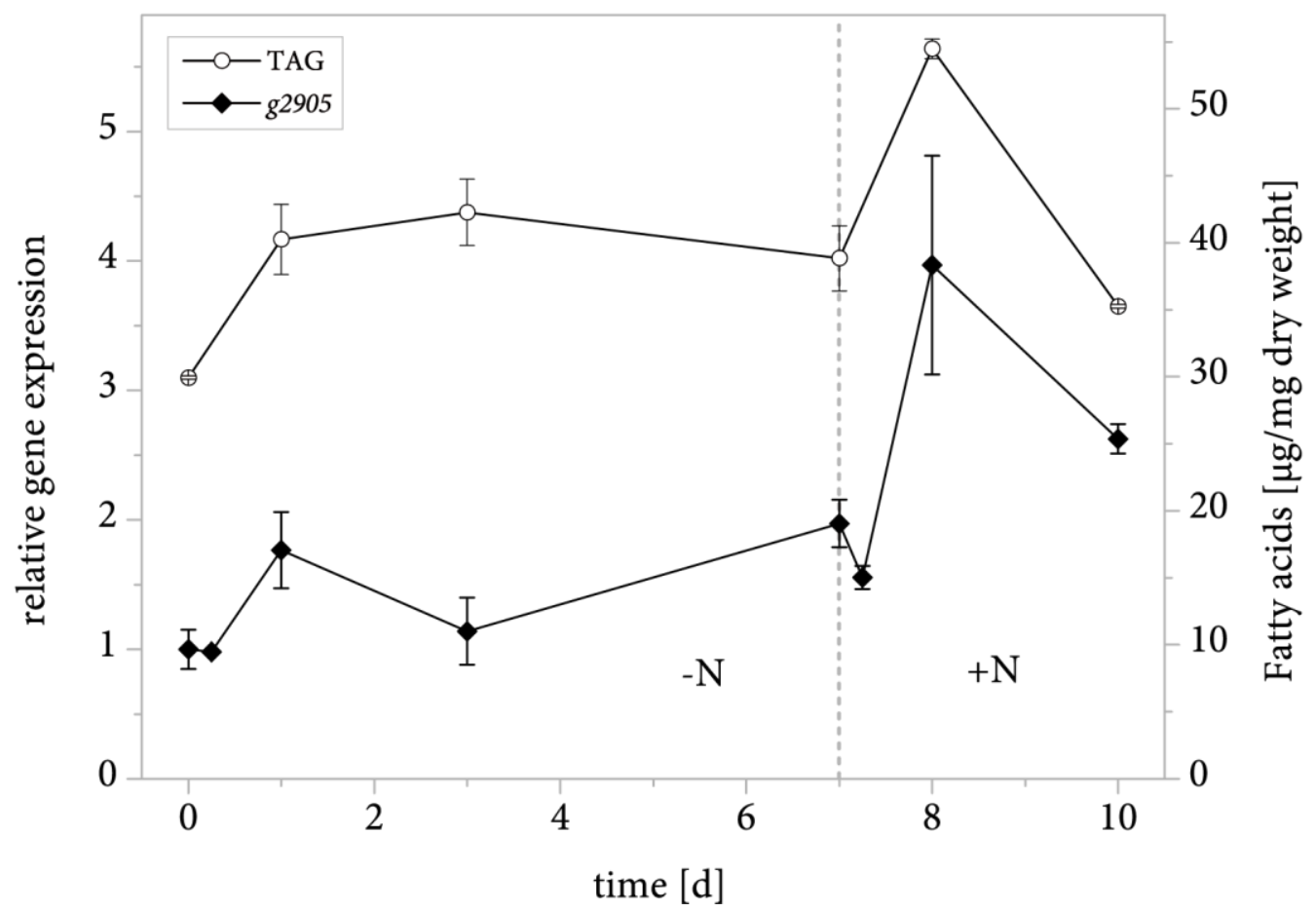

Figure 20: Expression of the L. incis a g2905 gene in response to changes in nitrogen supply. Transcript levels were determined by quantitative real-time PCR and normalized to RIBOSOMAL PROTEIN S21 transcripts. Expression is shown relative to time point 0 and error bars represent the standard error of the mean for three batches cultivated in parallel in a single experiment. The dotted line indicates nitrogen resupply and TAG levels are shown for comparison. 
Results

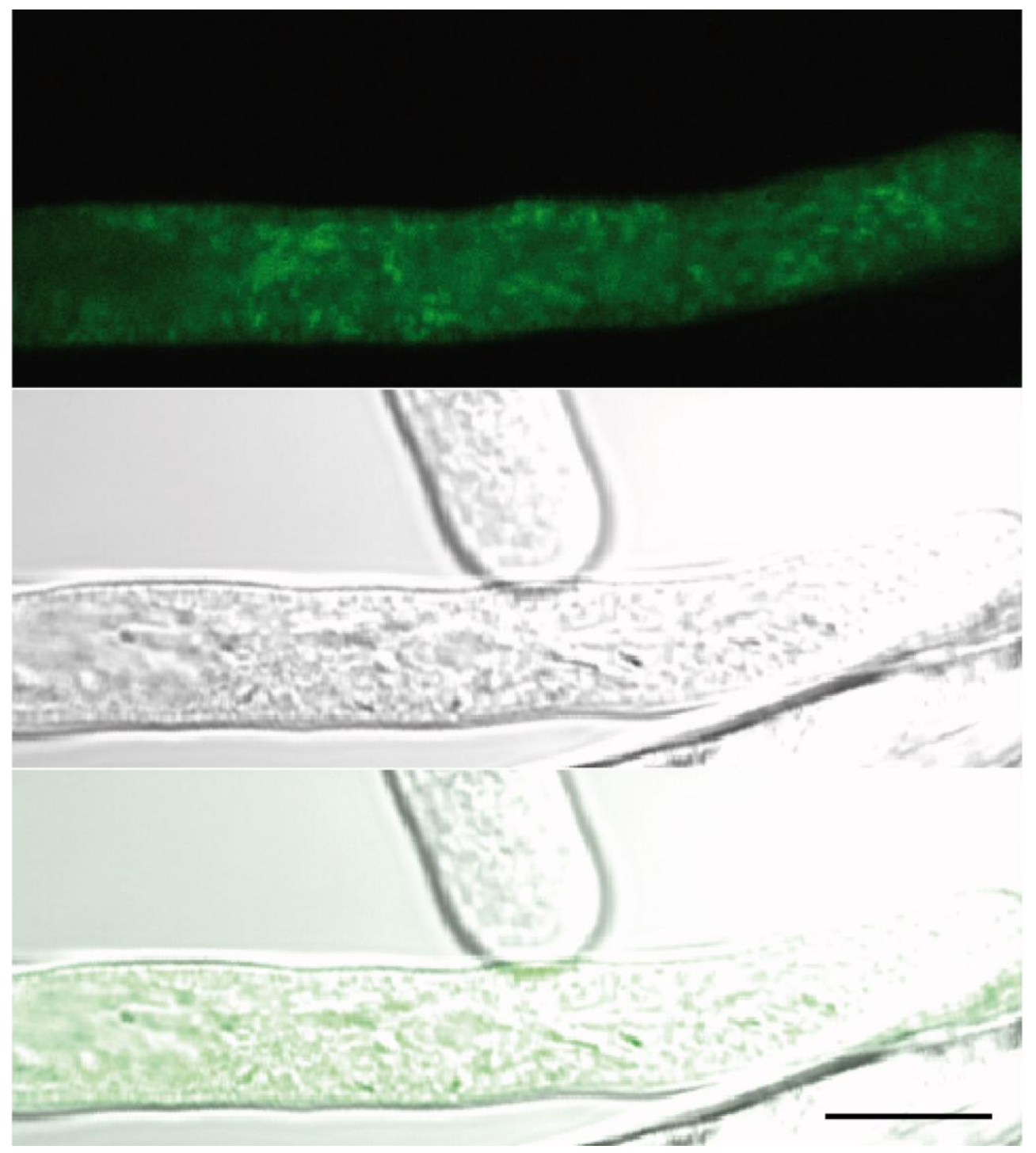

Figure 21: Subcellular localization of mVenus-L. incisa g2905 fusion in N. tabacum pollen tubes. Fluorescence was documented by confocal laser scanning microscopy after $6 \mathrm{~h}$ of pollen germination. From top to bottom: mVenus fluorescence, brightfield image, merged image. 9 out of 9 pollen tubes analyzed showed comparable results. Scale bar $=10 \mu \mathrm{m}$. 


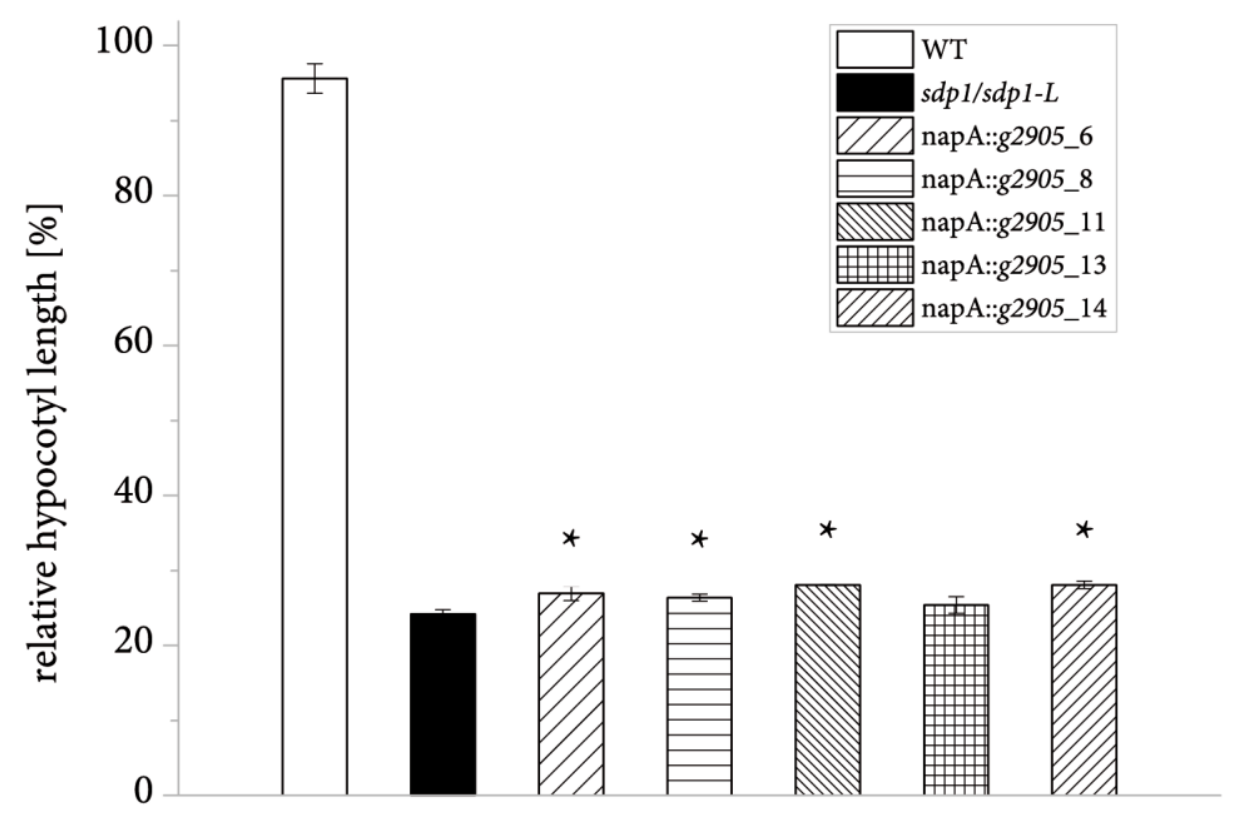

B

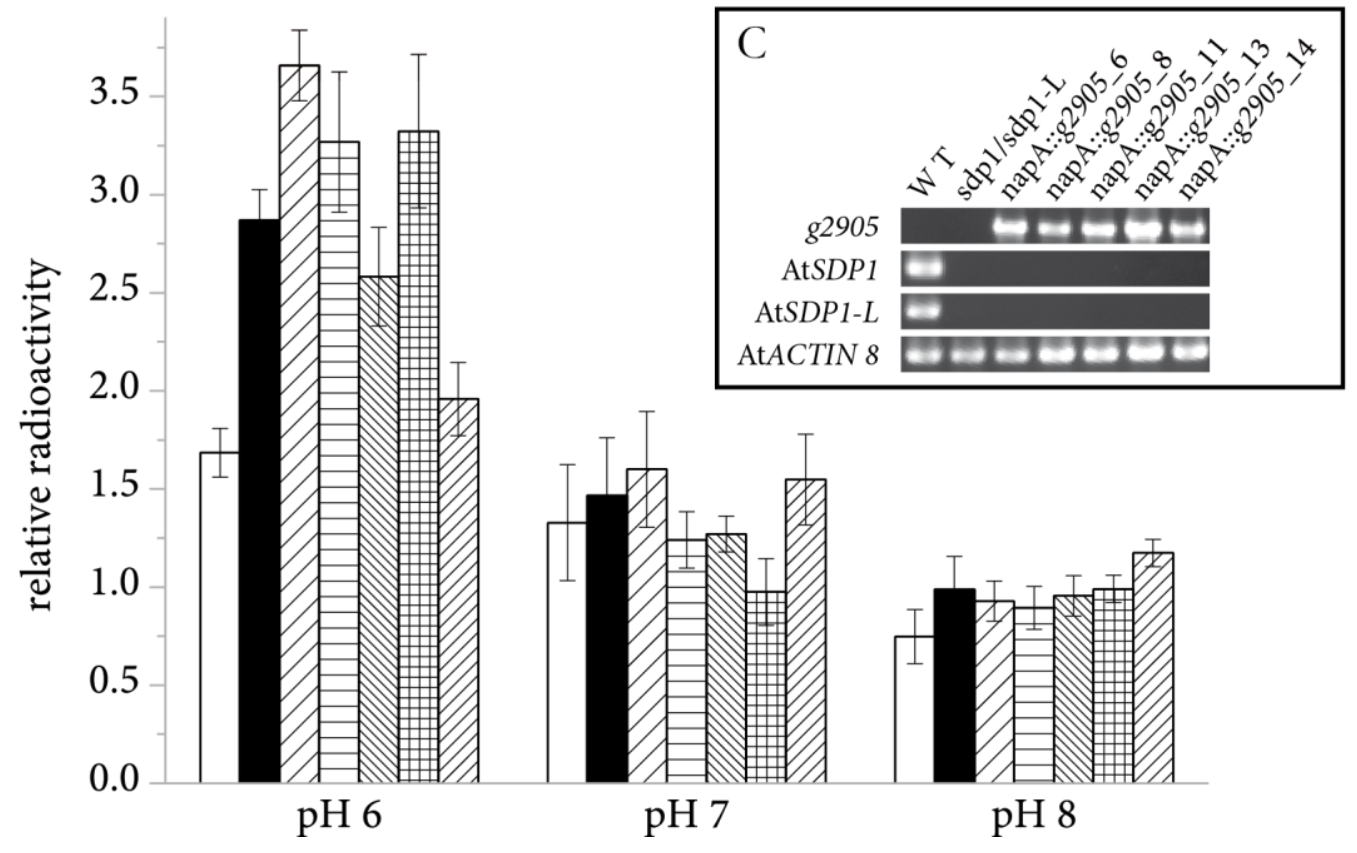


Figure 22: Lipase activity assays with L. incisa g2905 in A. thaliana. Sdp1/sdp1-L seedlings with the Brassica napus napinA (napA) promoter controlling expression of the L. incisa g2905 gene in five independent lines are compared to the mutant carrying an empty vector as well as the wildtype (WT). A, Hypocotyl length of seedlings after 5 d of germination in the dark on $1 / 2$ MS agar plates without sucrose relative to growth with sucrose. For each growth condition and independent line, three or four batches of at least 15 seedlings were measured in a single experiment and hypocotyl length of seedlings germinated without sucrose was divided by the average value for the same line with sucrose. Error bars represent the standard error of the mean. Asterisks denote measurements on transgenic lines deviating significantly from the mutant (two-sided Student's T-test, $\alpha=0.05$ ). B, Degradation of ${ }^{14} C$ labeled TAG in crude extracts of $3 \mathrm{~d}$ old seedlings. Following incubation in different buffers, the hexane extract of each reaction was separated by thin layer chromatography (TLC) and radioactivity was detected across the TLC plate. Relative radioactivity was calculated as the ratio of radiation emitted by TAG and free fatty acids as measured densitometrically. Error bars represent the standard deviation of three technical replicates in a single experiment. C, Confirmation of gene expression in dry seeds of independent lines.

\subsubsection{Characterization of the L. incisa SUGAR-DEPENDENT 1 lipase (LiSDP1)}

Another putative lipase encoded in the L. incis a genome exhibits a high degree of homology with the $A$. thaliana enzymes SUGAR-DEPENDENT 1 (AtSDP1) and SDP1-LIKE (AtSDP1-L) (Figure 23, Supplemental figure 4) and was therefore termed LiSDP1. The three proteins have a patatin domain in common, which contains a characteristic catalytic dyad of serine and aspartate, making them members of the diverse group of patatin domain lipases (Kienesberger et al., 2009). 


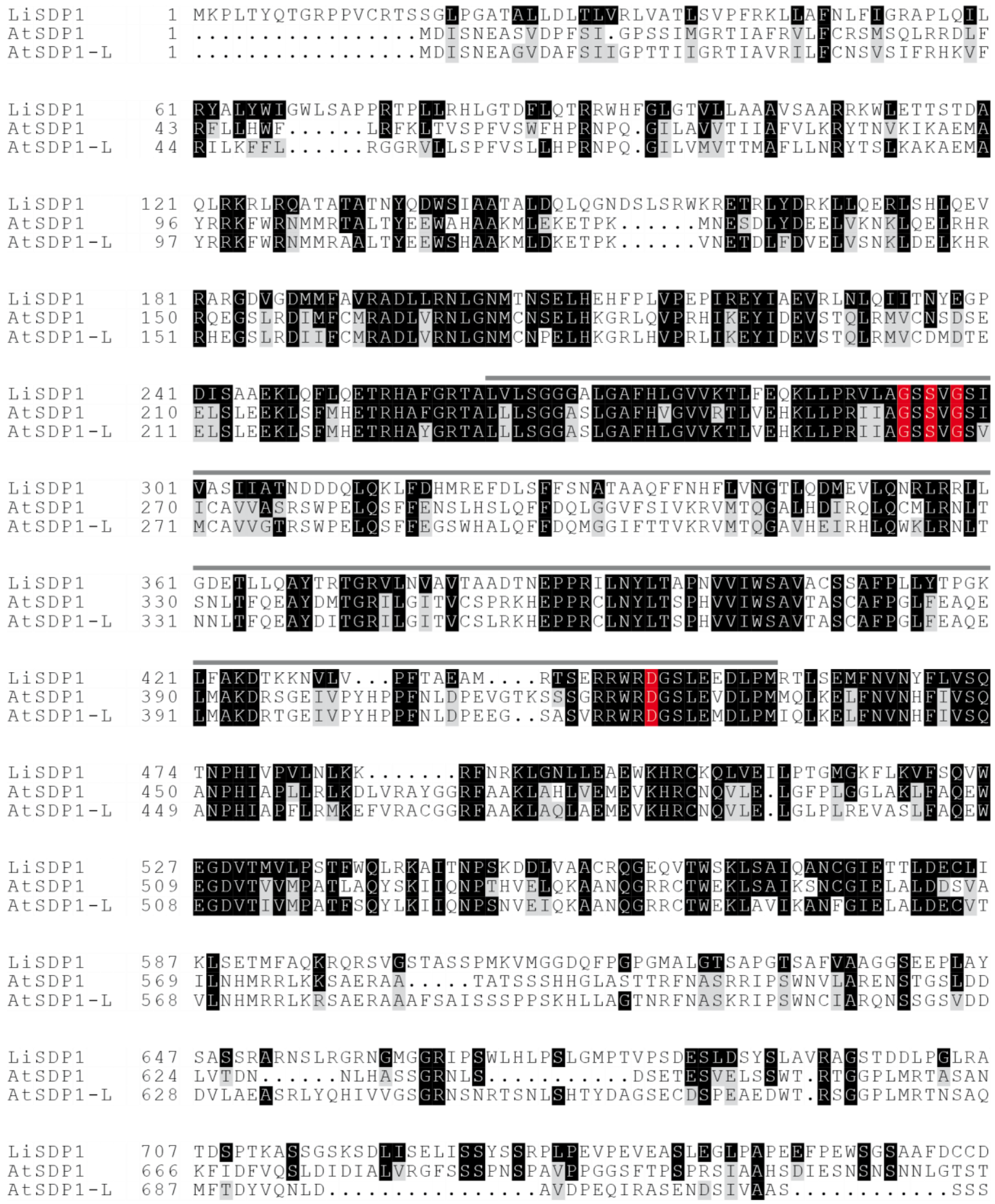

Figure 23: Features of the L. incisa SUGAR DEPENDENT 1 (LiSDP1) amino acid sequence (partial). Similarities with the amino acid sequences of A. thaliana SDP1 (AtSDP1) and SDP1-LIKE (AtSDP1-L) are shown with black and grey boxes highlighting identical and similar residues, respectively. Sequences were aligned using MultAlin and ExPASy BoxShade software and a section of the alignment is shown. Components of the active site are marked in red. The grey bar represents the conserved patatin domain. See Supplemental figure 4 for the full alignment. 
In L. incisa cultures starved of nitrogen, LiSDP1 gene expression increases more than 2-fold during the TAG accumulation phase and undergoes an additional rapid increase upon resupply of nitrogen (Figure 24). $3 \mathrm{~d}$ afterwards when the storage lipid has been degraded, gene expression once again reaches a level comparable to the beginning of the experiment.

Heterologous expression of the LiSDP1 gene with a fluorescent tag in N. tabacum pollen tubes results in a pattern of fluorescence that is reminiscent of AtSDP1 in A. thaliana seedlings (Figure 25, Eastmond, 2006).

Considering the strong sequence homology with well characterized $A$. thaliana TAG lipases, a possible functional complementation of a mutant line lacking these enzymes was investigated. As described above, the $s d p 1 / s d p 1-L$ mutant is hardly able to access its storage lipids to drive postgerminative growth. This phenotype becomes especially clear when depriving the mutant seeds of light and an external carbon source and comparing their hypocotyl elongation to that of wildtype seedlings. As shown in Figure 26, LiSDP1 can partially rescue

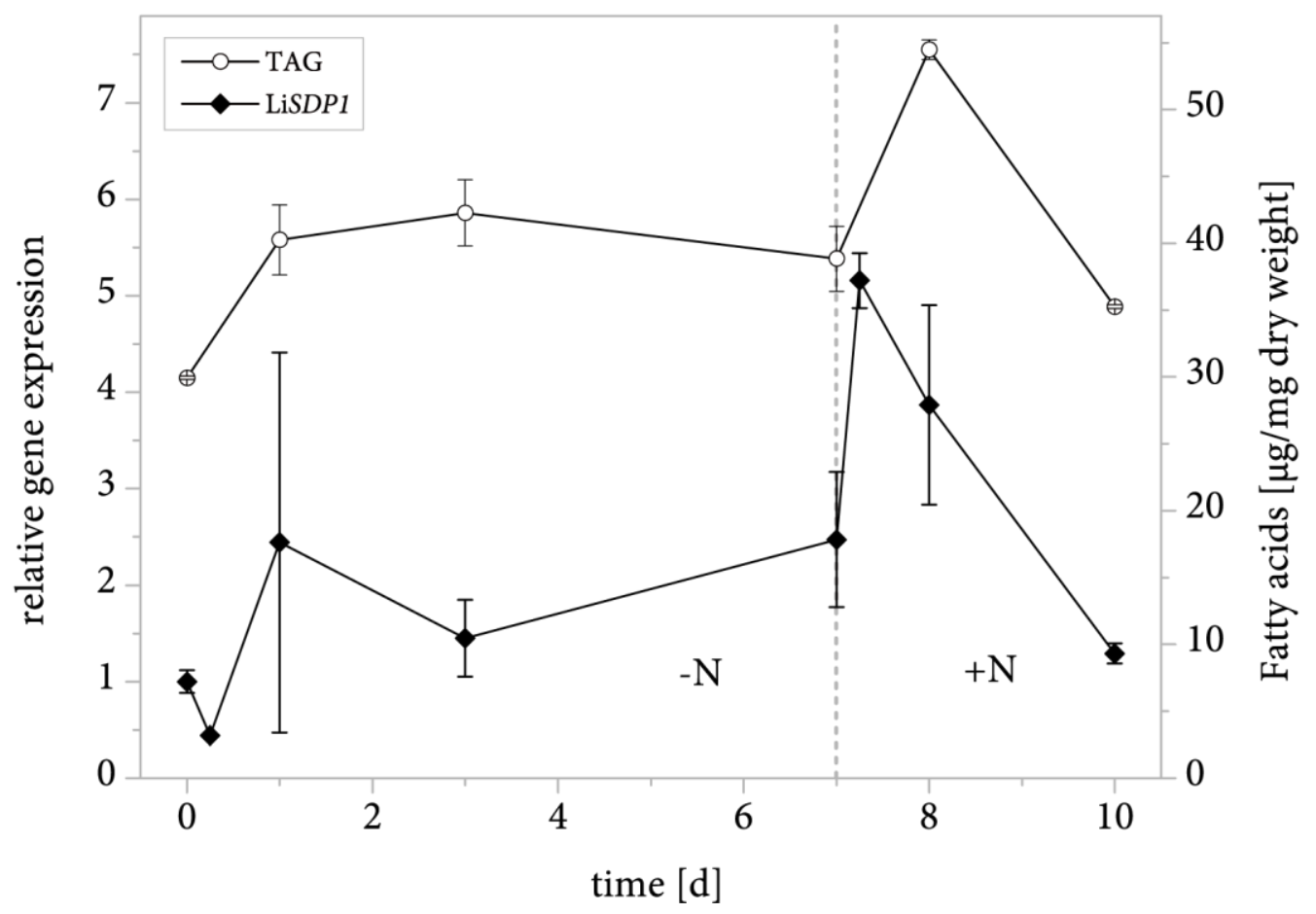

Figure 24: Expression of the L. incisa SUGAR DEPENDENT 1 (LiSDP1) gene in response to changes in nitrogen supply. Transcript levels were determined by quantitative real-time PCR and normalized to RIBOSOMAL PROTEIN S21 transcripts. Expression is shown relative to time point 0 and error bars represent the standard error of the mean for three batches cultivated in parallel in a single experiment. The dotted line indicates nitrogen resupply and TAG levels are shown for comparison. 


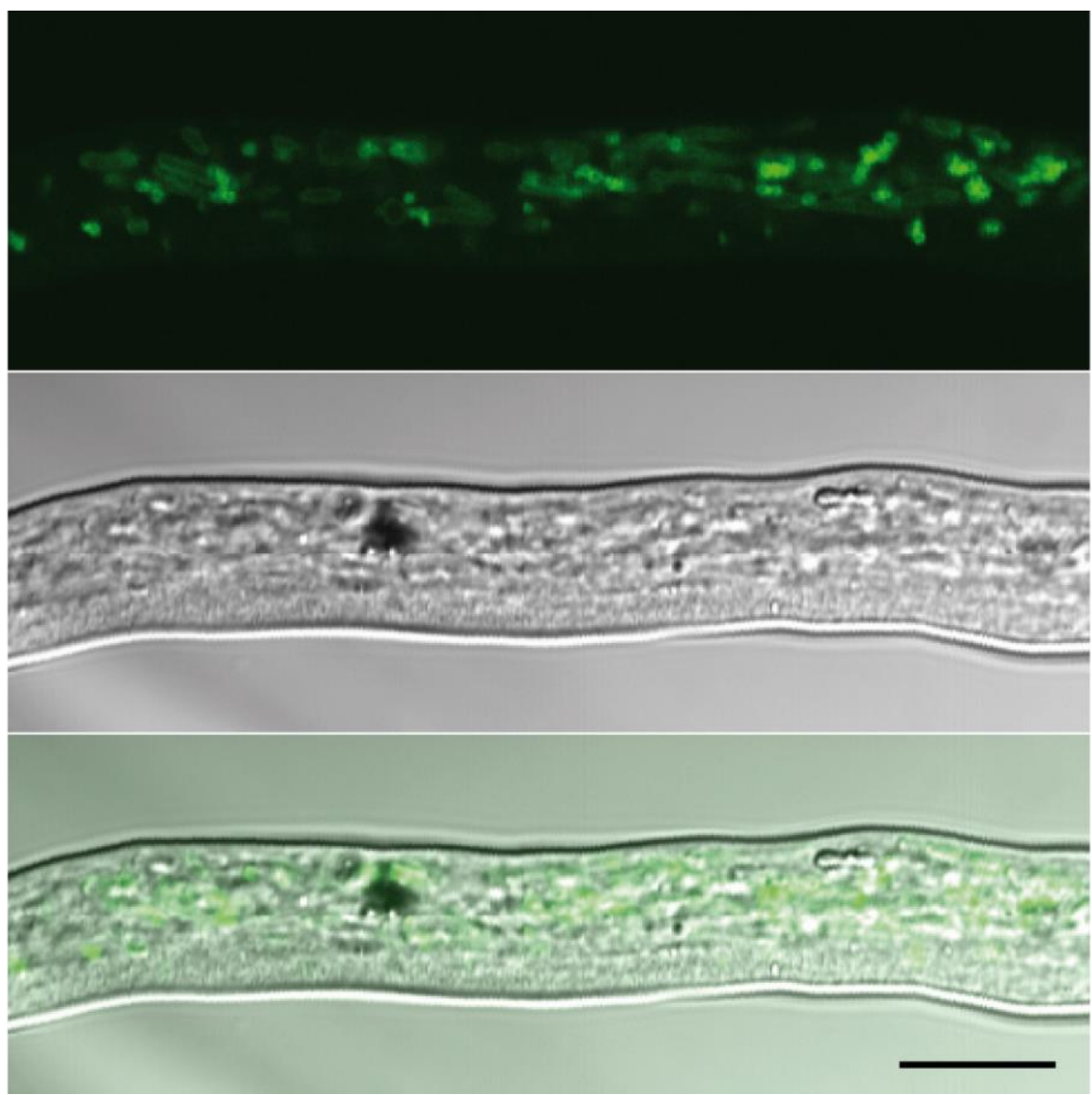

Figure 25: Subcellular localization of mVenus-LiSDP1 fusion in N. tabacum pollen tubes. Fluorescence was documented by confocal laser scanning microscopy after $6 \mathrm{~h}$ of pollen germination. From top to bottom: mVenus fluorescence, brightfield image, merged image. Five out of five pollen tubes analyzed showed comparable results. Scale bar $=10 \mu \mathrm{m}$.

postgerminative growth of the knockout mutant. The effect is more pronounced in lines constitutively expressing the algal gene under the control of the Cauliflower Mosaic Virus 35S promoter (35S, A) than with seed specific expression (B). Measurements for germination with and without sucrose are shown separately in Supplemental figure 5. 
A

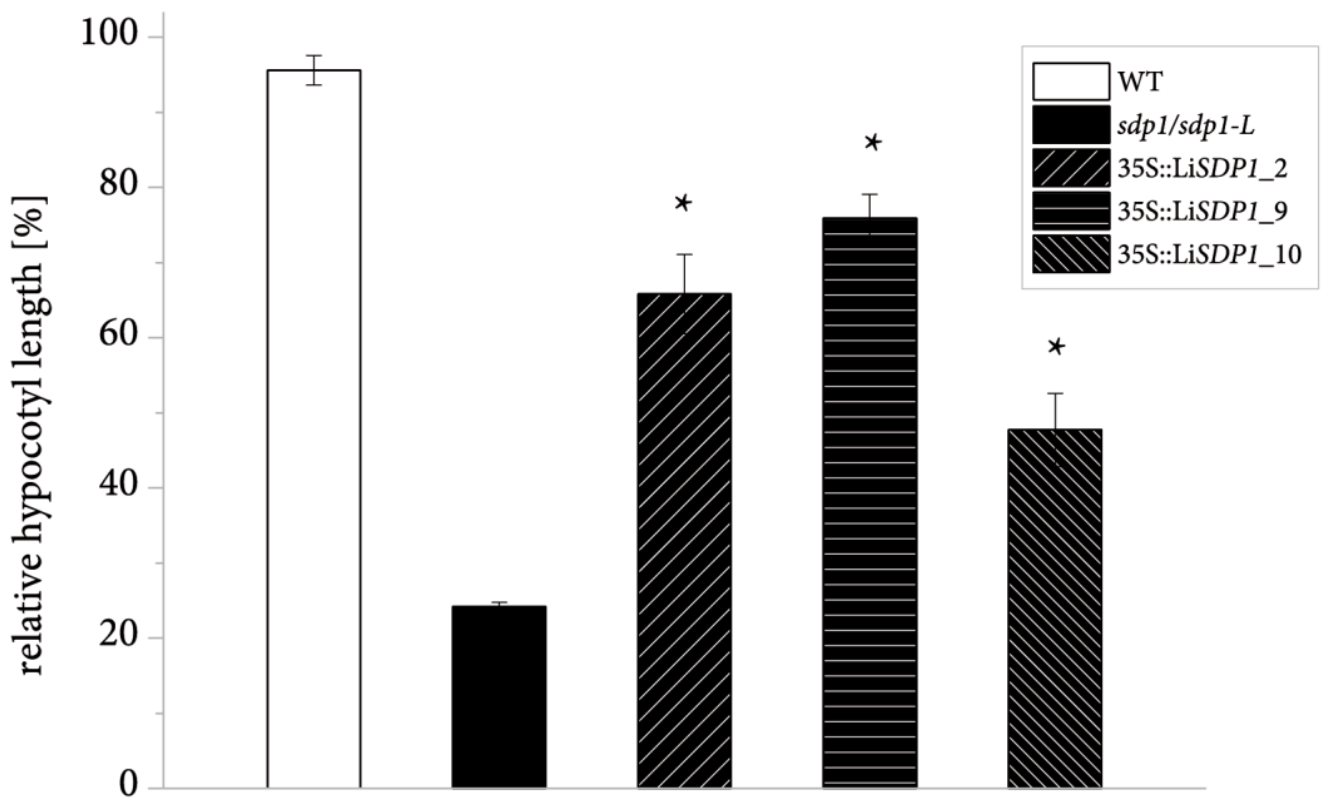

$\mathrm{B}$

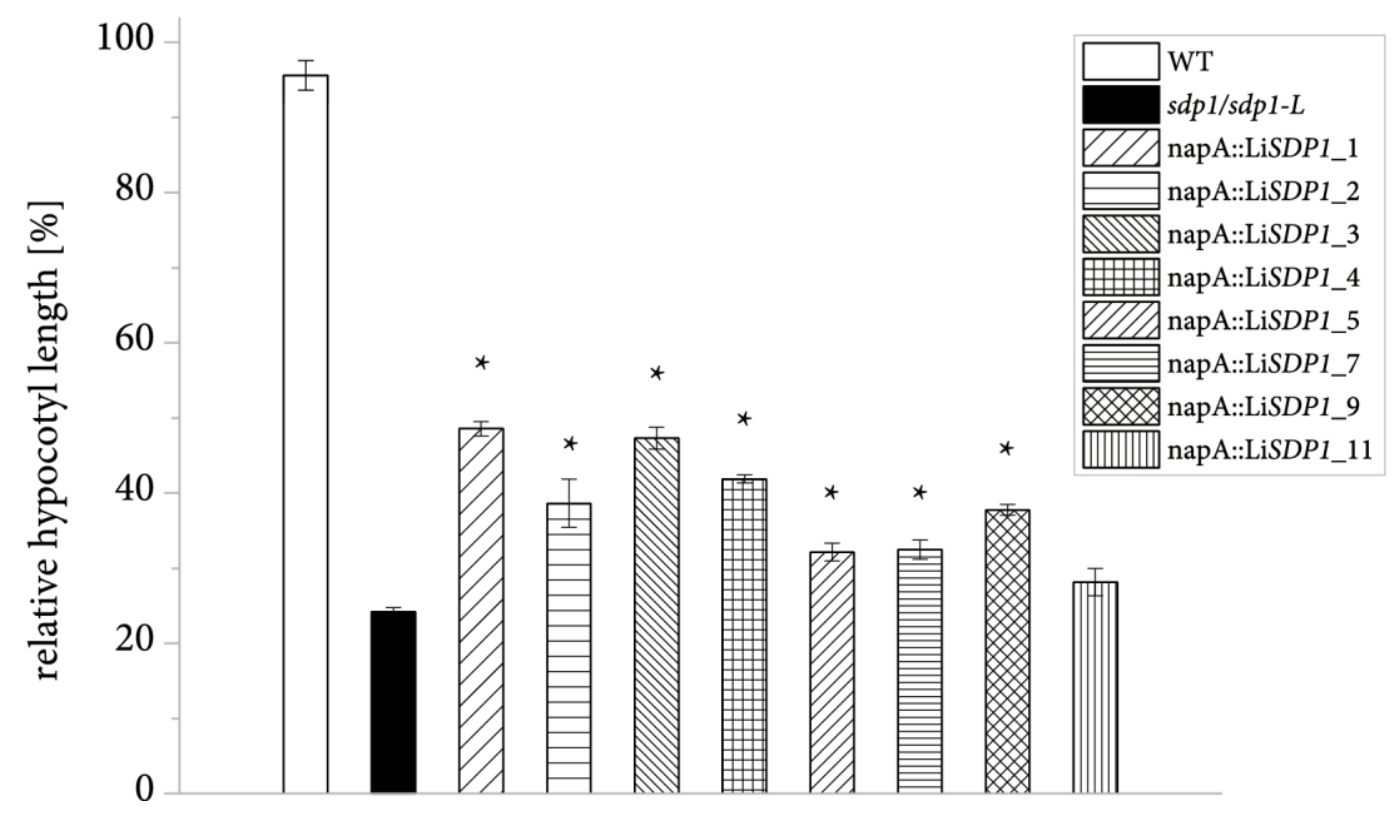

Figure 26: Effect of L. incisa SUGAR DEPENDENT 1 (LiSDP1) expression on seedling growth in A. thaliana. Sdp1/sdp1-L seedlings expressing the LiSDP1 gene in independent lines are compared to the mutant carrying an empty vector as well as the wildtype (WT). Following $5 \mathrm{~d}$ of germination in the dark on $1 / 2$ MS agar plates without sucrose, hypocotyl length was determined relative to growth with sucrose. For each growth condition and independent line, three or four batches of at least 15 seedlings were measured and hypocotyl length of seedlings germinated without sucrose was divided by the average value for the same line with sucrose. Error bars represent the standard error of the mean in a single experiment. Asterisks denote measurements on transgenic lines deviating significantly from the mutant (two-sided Student's T-test, $\alpha=0.05$ ). A, Cauliflower Mosaic Virus $35 S$ (35S) promoter controlling expression of the LiSDP1 gene. B, Brassica napus napinA (napA) promoter controlling expression of the LiSDP1 gene. 
The TFA content per 5 day-old etiolated seedling was determined by gas chromatography and is depicted relative to the seed fatty acid content in Figure 27. Wildtype seedlings only contain $10 \%$ of the initial lipids in seeds as measured in TFA content, whereas the double knockout mutant retains $80 \%$ of the seed amount. Five out of 7 lines analyzed show a clear reduction in fatty acid content when compared to the mutant. Among the plant lines constitutively expressing the algal gene, a higher degree of fatty acid breakdown appears to result in longer hypocotyls, a relation that cannot be seen as clearly for seed specific expression (Figures 26 and 27).

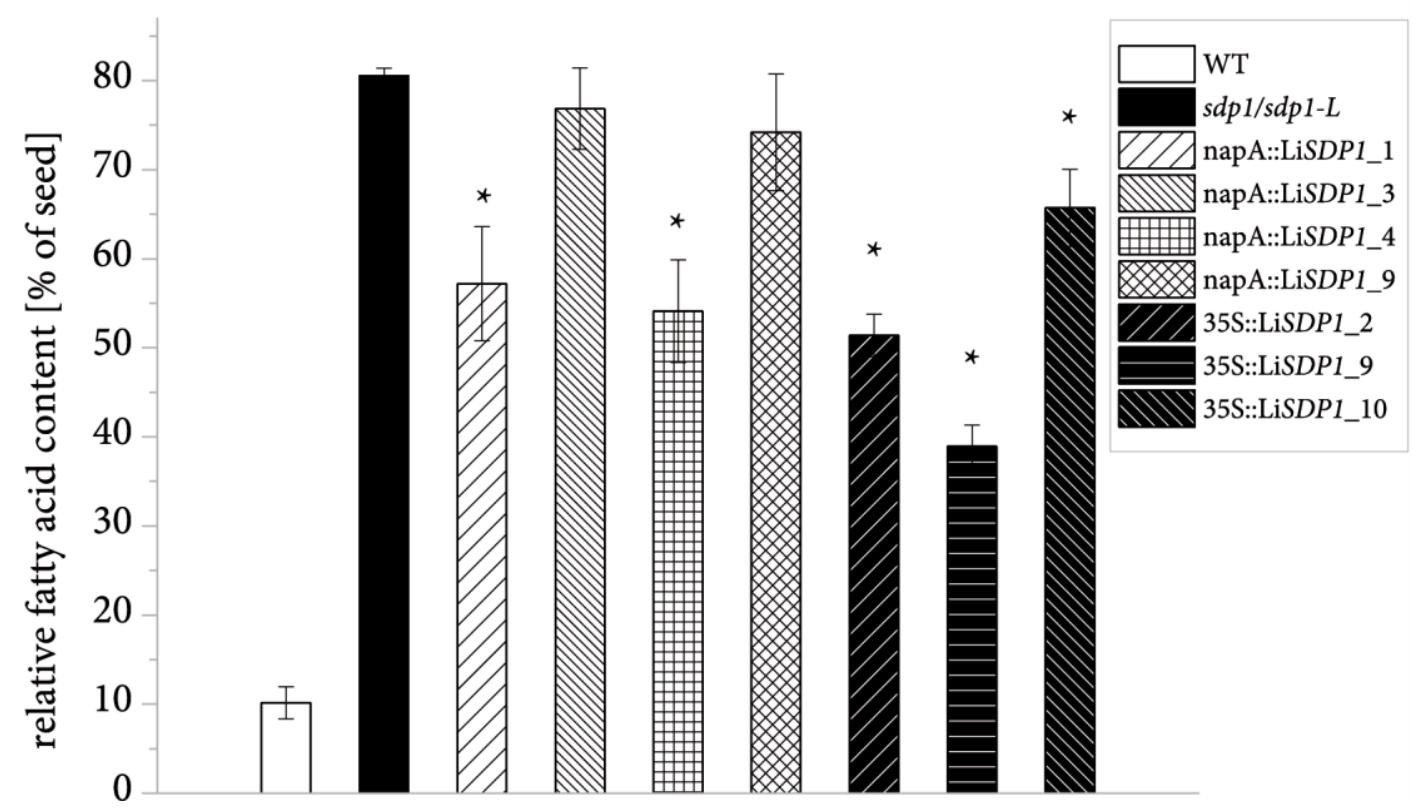

Figure 27: Effect of L. incisa SUGAR DEPENDENT 1 (LiSDP1) expression on TFAs in A. thaliana etiolated seedlings. The TFA content per seedling is shown relative to the amount per seed and the sdp1/sdp1-L mutant expressing the LiSDP1 gene in independent lines is compared to the mutant carrying an empty vector as well as the wildtype (WT). Following $5 \mathrm{~d}$ of germination in the dark on $1 / 2$ MS agar plates without sucrose, TFAs were derivatized by acidic methanolysis and analyzed by gas chromatography. For each independent line, three or four batches of 10 seeds and three or four batches of at least 9 seedlings were measured and the seedling fatty acid content was divided by the average value for seeds of the same line. Error bars represent the standard error of the mean in a single experiment. Asterisks denote measurements on transgenic lines deviating significantly from the mutant (two-sided Student's T-test, $\alpha=0.05$ ). 
The effect of LiSDP1 activity on the composition of fatty acids in the seedlings was also investigated. For this purpose, the proportion of each fatty acid species in the seedlings was related to the proportion in seeds. Concerning the fate of saturated fatty acids, the endogenous A. thaliana TAG lipases have the strongest effect on 20:0, as the difference between wildtype and mutant figures is largest for this fatty acid (Figure 28A). A similar observation cannot be made for LiSDP1 activity, since most complemented lines behave similarly with respect to the individual saturated fatty acid species. With regards to monounsaturated fatty acids, AtSDP1 and AtSDP1-L activity appear to affect 20:1, a marker fatty acid of TAG in $A$. thaliana seeds, to a larger extent than 18:1, a tendency that is not visible for the L. incisa homolog (Figure 28B). Finally, the measurements of PUFAs do not point to a distinct effect of the $A$. thaliana or L. incisa lipases (Figure 29). 
A

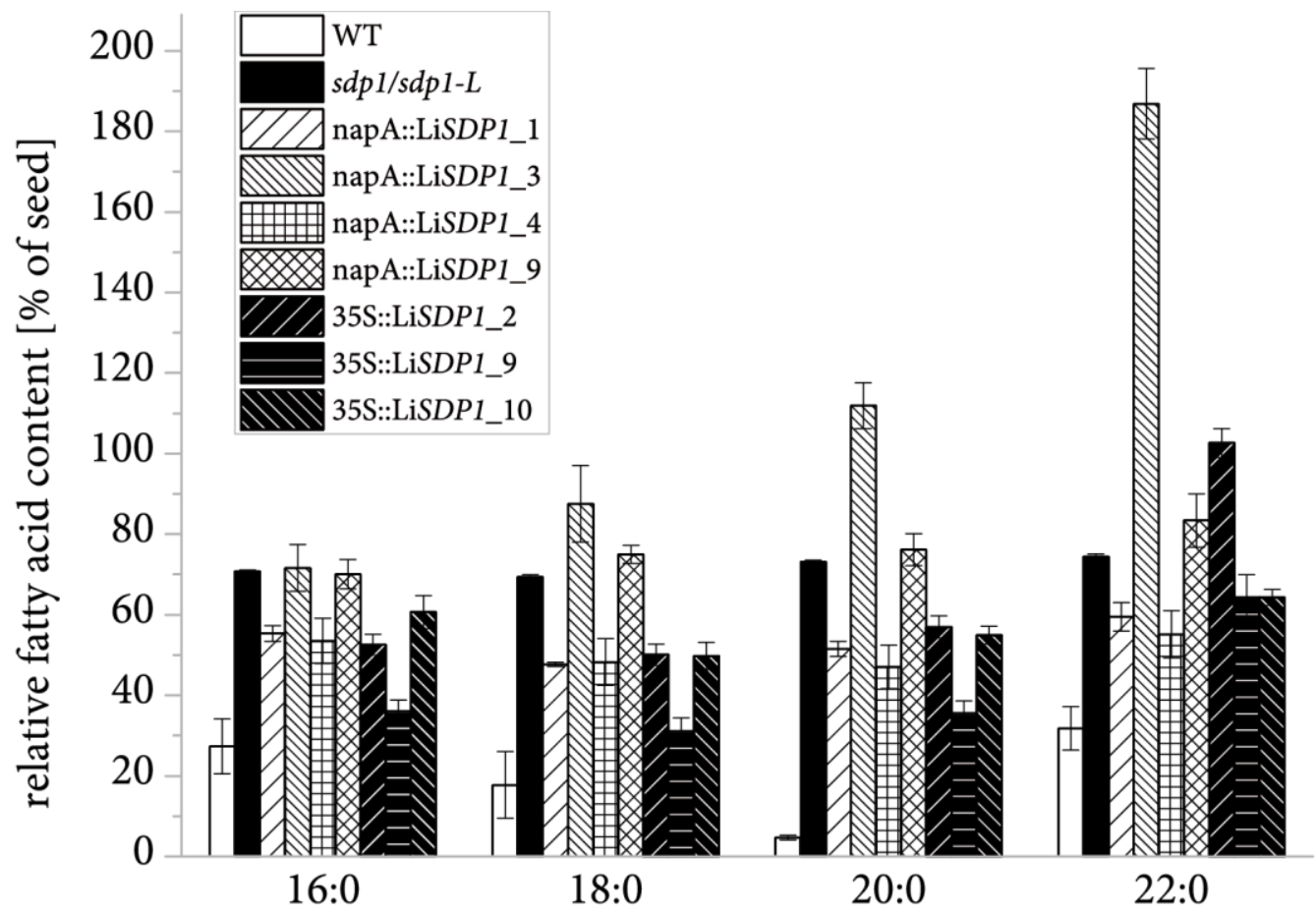

B

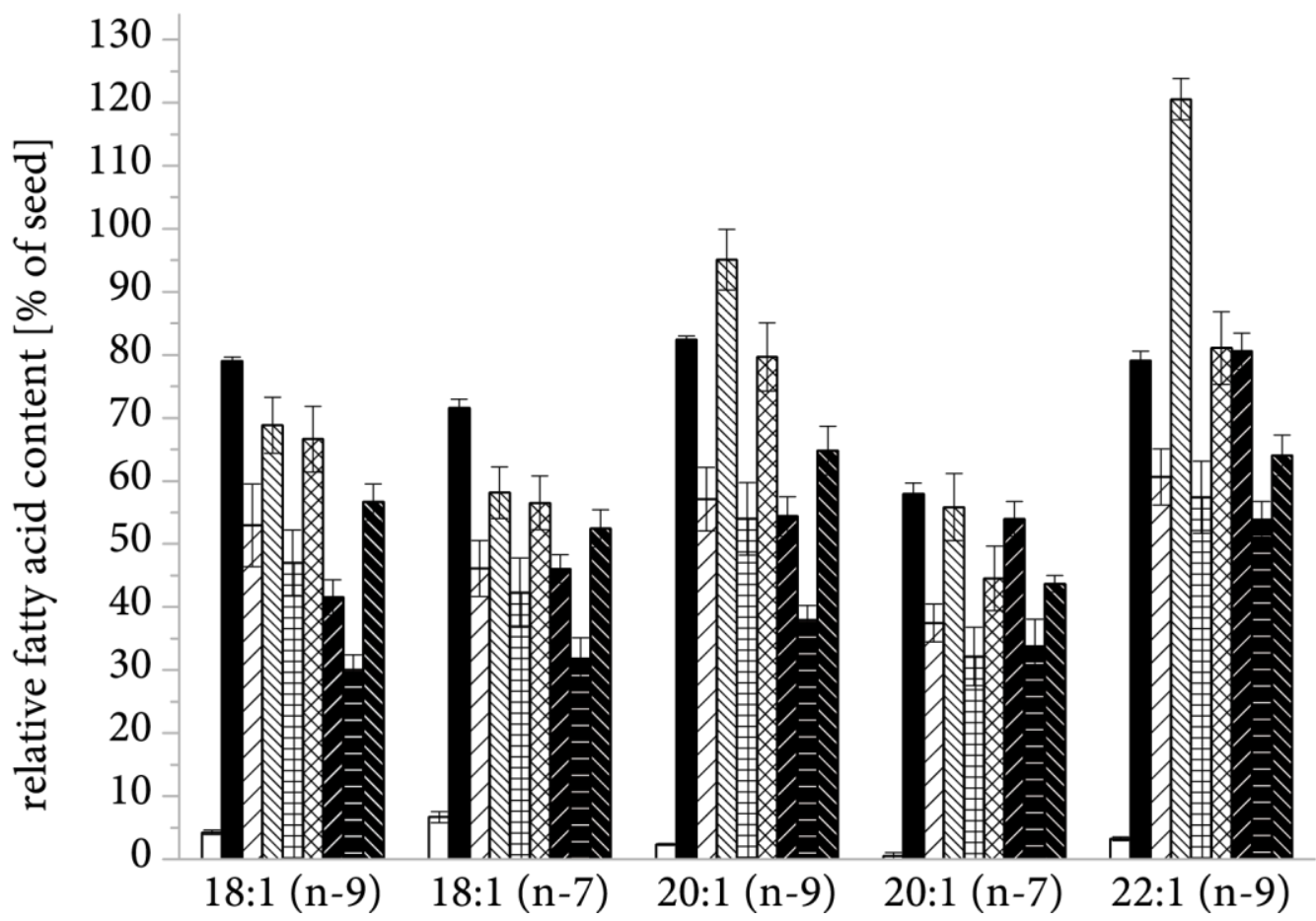


Figure 28: Effect of L. incisa SUGAR DEPENDENT 1 (LiSDP1) expression on saturated and monounsaturated fatty acids in $A$. thaliana etiolated seedlings. For each fatty acid, the amount per seedling is shown relative to the amount per seed and the sdp1/sdp1-L mutant expressing the LiSDP1 gene in independent lines is compared to the mutant carrying an empty vector as well as the wildtype (WT). Following $5 d$ of germination in the dark on $1 / 2$ MS agar plates without sucrose, TFAs were derivatized by acidic methanolysis and analyzed by gas chromatography. For each independent line, three or four batches of 10 seeds and three or four batches of at least 9 seedlings were measured and the seedling fatty acid content was divided by the average value for seeds of the same line. Error bars represent the standard error of the mean in a single experiment. A, saturated fatty acids. $B$, Monounsaturated fatty acids.

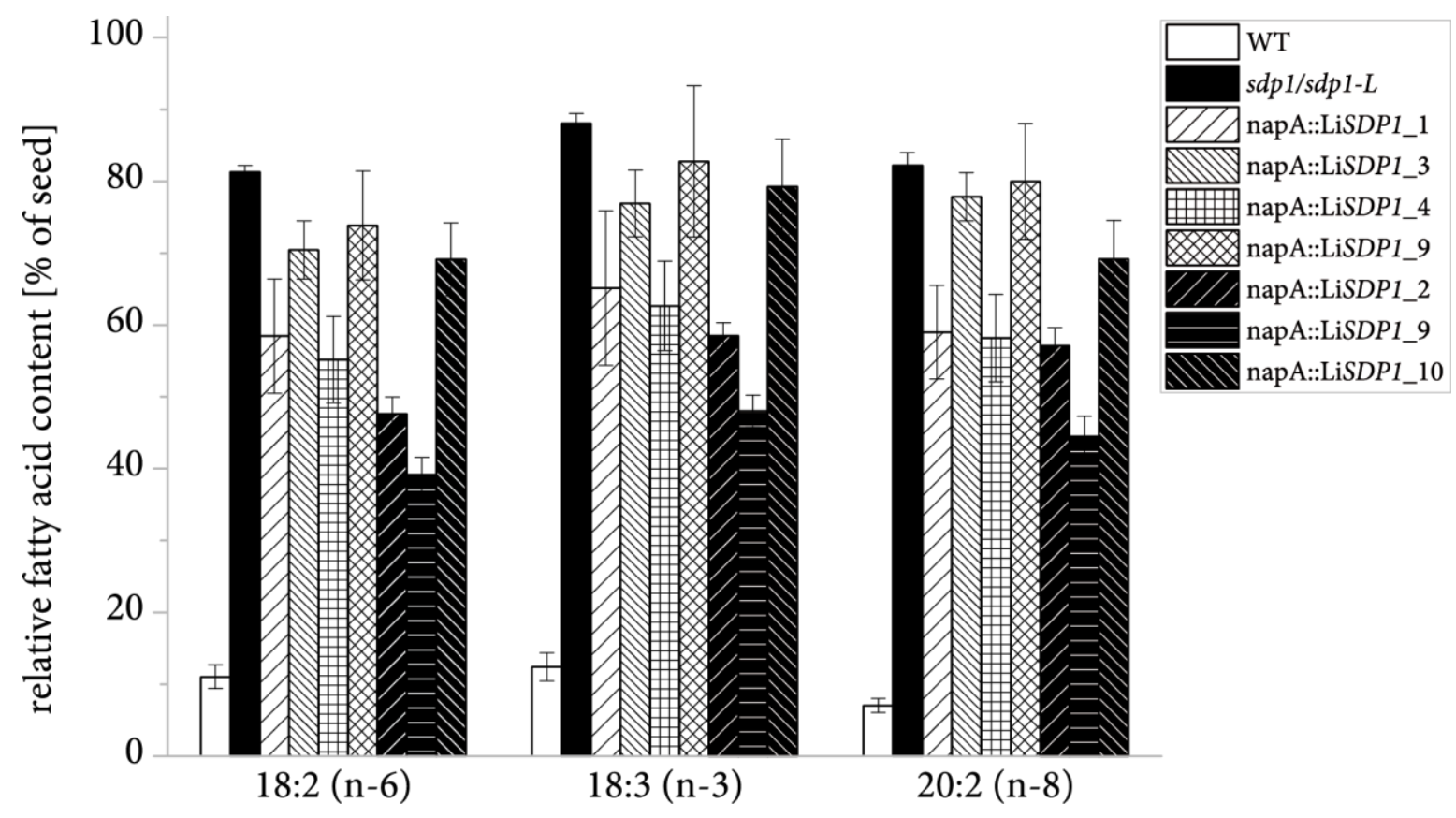

Figure 29: Effect of L. incisa SUGAR DEPENDENT 1 (LiSDP1) expression on PUFAs in A. thaliana etiolated seedlings. For each fatty acid, the amount per seedling is shown relative to the amount per seed and the sdp1/sdp1-L mutant expressing the LiSDP1 gene in independent lines is compared to the mutant carrying an empty vector as well as the wildtype (WT). Following 5 d of germination in the dark on $1 / 2$ MS agar plates without sucrose, TFAs were derivatized by acidic methanolysis and analyzed by gas chromatography. For each independent line, three or four batches of 10 seeds and three or four batches of at least 9 seedlings were measured and the seedling fatty acid content was divided by the average value for seeds of the same line. Error bars represent the standard error of the mean in a single experiment. 


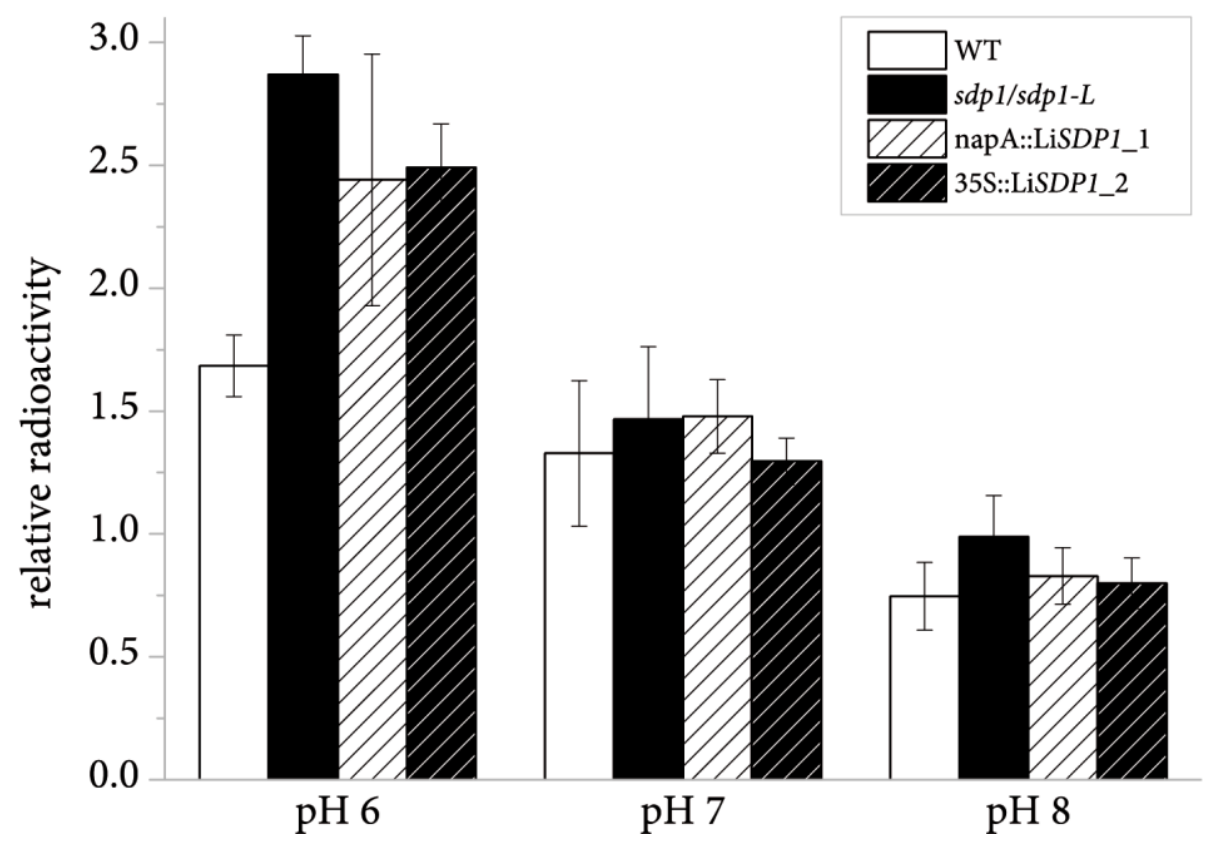

Figure 30: Effect of L. incisa SUGAR DEPENDENT 1 (LiSDP1) expression on TAG degradation in transgenic $A$. thaliana seedlings. Sdp1/sdp1-L seedlings with the Brassica napus napinA (napA) promoter or the Cauliflower Mosaic Virus 355 (35S) promoter controlling expression of LiSDP1 are compared to the mutant carrying an empty vector as well as the wildtype (WT). Crude extracts of $3 a$ old seedlings were incubated with ${ }^{14} \mathrm{C}$ labeled TAG in different buffers, the hexane extract of each reaction was separated by thin layer chromatography (TLC) and radioactivity was detected across the TLC plate. Relative radioactivity was calculated as the ratio of radiation emitted by TAG and free fatty acids as measured densitometrically. Error bars represent the standard deviation of three technical replicates in a single experiment.

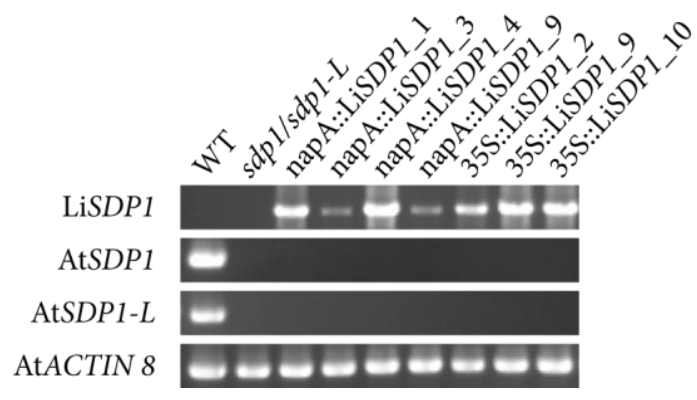

Figure 31: Confirmation of gene expression. Transcripts were detected in dry seeds of independent lines with the Brassica napus napin A (napA) promoter or the Cauliflower Mosaic Virus 355 (35S) promoter controlling expression of L. incis a SUGAR DEPENDENT 1 (LiSDP1).

In addition to the assessment of hypocotyl growth, fatty acid content and -composition, TAG hydrolysis was also investigated by monitoring ${ }^{14} \mathrm{C}$ TAG degradation in crude extracts of 
complemented $A$. thaliana sdp1/sdp1-L seedlings as described in section 4.2 .4 (Figure 30). Even though a pH optimum of 8 has been determined for AtSDP1 (Eastmond, 2006), the ratio of TAG and free fatty acids does not differ significantly between wildtype and mutant seedlings in this condition and the same is true for $\mathrm{pH}$ 7. The difference in TAG degradation is much more pronounced at a more acidic $\mathrm{pH}$ and for all three reaction conditions, total TAG lipase activity is in the range of the mutant.

The same plant lines were used for all activity assays described in this section and gene expression was confirmed in dry seeds as shown in Figure 31. 


\section{Discussion}

In this study, the green microalga L. incisa was analyzed with respect to accumulation and mobilization of ARA-rich TAG stored in LBs, processes that are strongly modulated by nitrogen availability. Special emphasis was placed on proteins that associate with LBs or may be involved in TAG hydrolysis.

\subsection{Lobosphaera incisa physiology is strongly affected by nitrogen supply}

Removal of nitrogen from the growth media severely affects algal metabolism and induces growth arrest (Cheng-Wu et al., 2002; Davidi et al., 2012; Msanne et al., 2012; Tsai et al., 2014). In the unicellular green microalga L. incisa, this radical change in cellular physiology is closely linked to an increase in the ratio of carotenoids to total chlorophyll (Simionato et al., 2013; Solovchenko et al., 2008b). The shift in pigment composition was confirmed in this study, during which three L. incisa cultures were subjected to nitrogen starvation for $7 \mathrm{~d}$ (Figure 1). It can mostly be attributed to the degradation of chloroplast membranes linked to a reduction in chlorophyll, a phenomenon commonly observed in microalgae in nitrogen depletion (Allen et al., 2015; Davidi et al., 2012; Dong et al., 2013a; Martin et al., 2014; Msanne et al., 2012; Peled et al., 2011; Schmollinger et al., 2014; Simionato et al., 2013). The ratio has been established to closely correlate with ARA accumulation in this alga (Solovchenko et al., 2009), a trend that could equally be observed in this study.

During the first $3 \mathrm{~d}$ of nitrogen limitation, the alga accumulates TAG up to $56 \%$ of TFAs, an intermediate level compared to what has been reported for the same strain in logarithmic and stationary growth (43 and $77 \%$, respectively, Bigogno et al., 2002a) or an extended period of nitrogen depletion (Khozin-Goldberg et al., 2002) and for other oleaginous microalgae cultivated in nitrogen limiting conditions (Allen et al., 2015; Li et al., 2014; Martin et al., 2014; Moellering and Benning, 2010; Msanne et al., 2012; Simionato et al., 2013). The overall amount of fatty acids determined in this experiment (and another one, data not shown) of $75 \mu \mathrm{g} / \mathrm{mg}$ dry weight however is less than half of the values published for comparable growth conditions (Solovchenko et al., 2008b) and remains constant during four more days of continuing nitrogen limitation, whereas it has been reported to double in this time in this strain (Solovchenko et al., 2008b), the closely related species C. subellipsoidea (Allen et al., 2015) and other green algae (Dong et al., 2013b; Msanne et al., 2012; Simionato et al., 2013; Xin et al., 2010). Small 
variations in cultivation conditions such as light quality and intensity, batch size or the exact state of the starter culture may be the cause for these deviations from data that have been previously published for L. incisa.

This alga is particularly interesting in terms of its fatty acid profile, as it has been accredited with the highest ARA content of any plant or alga (Bigogno et al., 2002a). Indeed, in this study ARA initially makes up $22 \%$ of the total and the proportion increases to $29 \%$ after $3 \mathrm{~d}$ without nitrogen (Figures 2 and 3), a level that is superior to many other oleaginous algae (Bigogno et al., 2002a; Li et al., 2014; Martin et al., 2014; Moellering and Benning, 2010; Msanne et al., 2012; Simionato et al., 2013), nevertheless the amount previously described is not reached (Solovchenko et al., 2008b). Although the ratio of carotenoids to chlorophyll as an indicator of the physiological state of the culture is in agreement with previously reported levels (Solovchenko et al., 2008b), total lipid content and TAG accumulation may have been affected by slightly altered growth conditions in this study. A complete fatty acid profile has not been described for L. incisa under nitrogen starvation in a comparable time frame, but a general decrease in the ratio of C16 and C18 fatty acids has also been observed in the closely related alga Coccomyxa subellipsoidea (Allen et al., 2015; Msanne et al., 2012) and in C. reinhardtii neutral lipids (Moellering and Benning, 2010).

In contrast to the effects of nitrogen limitation, the physiological response to nitrogen resupply after a period of nitrogen limitation has not been studied in many algae. In this study, the ratio of carotenoids and chlorophyll was found to no longer correlate with the total amount of fatty acids which decrease, but a similar trend as that of the storage lipid TAG can be observed. The amount of fatty acids sequestered in this lipid class rises to $90 \%$ of overall fatty acids during $1 \mathrm{~d}$ before decreasing, an observation that has not been made in C. subellipsoidea or in C. reinhardtii, which experience a sharp drop in TAG level within 24 h (Allen et al., 2015; Tsai et al., 2014).

This could be explained for instance by phospholipid:diacylglycerol acyltransferase (PDAT) and TAG lipase activities not responding to the change in growth conditions as rapidly as other cellular processes. This was not investigated in this study, but continued transfer of fatty acids, especially ARA, from membrane lipids to diacylglycerol (DAG) along with a lag in TAG hydrolysis would result in the observed accumulation of ARA enriched TAG. As the C. reinhardtii PDAT has been shown to use not only phospholipids but also the thylakoid membrane lipid MGDG as an acyldonor to synthesize TAG (Yoon et al., 2012), this delay in 
adaptation could also explain the minor transient increase in the ratio of carotenoids and chlorophyll occurring simultaneously. Transcript data for $C$. reinhardtii indicate only a minor decrease in PDAT expression $48 \mathrm{~h}$ after nitrogen resupply (Tsai et al., 2014 supplemental information), while earlier responses to the change in growth conditions have so far not been analyzed on a transcript level in this or any other alga.

TAG levels in L. incisa have previously been shown to be reduced after $2 \mathrm{~d}$ of recovery in nitrogen replete media (Khozin-Goldberg et al., 2005), but data for intermediate time points are not available. The reduction in 18:1 (n-9) in both TAG and overall fatty acids as well as the increasing proportion of ARA in TAG observed in this experiment is in line with the data published previously (Khozin-Goldberg et al., 2005).

\subsection{Nitrogen supply affects expression of genes commonly used for normalization in qRT-PCR}

qRT-PCR requires normalization of transcript levels to a gene that is stably expressed across the tissues, developmental stages and treatments to be compared (Kozera and Rapacz, 2013; Løvdal and Lillo, 2009). For a unicellular organism that responds to a stress by entering a quiescent state, the choice of reference gene must be especially carefully taken, as even housekeeping functions may be affected (Schmollinger et al., 2014). Indeed, the genes putatively encoding ACTIN and RECEPTOR FOR ACTIVATED PROTEIN KINASE C, which are frequently used to normalize transcript levels in studies of $C$. reinhardtii under nutrient deprivation (Allen et al., 2007; Boyle et al., 2012; Tsai et al., 2014), are differentially expressed (unpublished RNAseq data).

Since RNAseq is not dependent on a single gene for normalization, previously published transcript data were used to identify stably expressed genes. To this end, a study in which the response of wildtype $C$. reinhardtii cultures to nitrogen starvation was compared to that of a mutant impaired in the regulation of cellular quiescence was searched for stably expressed genes (Tsai et al., 2014 supplemental information). Using qRT-PCR, homologs of these genes in L. incisa were tested for expression at several time points during a $7 \mathrm{~d}$ period of nitrogen deprivation followed by $3 \mathrm{~d}$ in replete media (Figure 4). Variability among the three biological replicates is commonly observed in this type of experiment (Boyle et al., 2012; Guihéneuf et al., 2011; Moellering and Benning, 2010) and can possibly be attributed to minor differences in 
$\mathrm{CO}_{2}$ supply and evaporation caused by slightly uneven diameters of aeration tubes. Not having undergone normalization themselves, these data are a direct representation of the qRT-PCR results. For each gene investigated, the average transcript level was relatively stable after the first $3 \mathrm{~d}$ in comparison to the onset of nitrogen starvation, thus being in accordance with the RNAseq data for $C$. reinhardtii. However, considerable variation occurred at intermediate and later time points as well as directly following the second change in growth media. Two possible explanations for this observation seem plausible:

Firstly, stress-related differences in the physiological state of the cultures may have led to unequally efficient RNA extraction or cDNA synthesis. The former could be due to easier breakage of cells under certain conditions and the latter may have been affected by stress-induced accumulation of metabolites that inhibit reverse transcriptase activity. In this case, it can be assumed that all transcripts were equally affected and the genes analyzed here are appropriate for use as references.

Secondly, the transitions into and out of cellular quiescence are likely to have had an effect on gene expression of the protein synthesis machinery. If this was the crucial underlying effect, normalization to these genes would not be valid, as not all transcriptional programs can be assumed to be affected in the same way. This notion is opposed by the observation that transcript levels for all genes analyzed in this study follow a comparable trend whether the data have been normalized or not (Supplemental Figures 6, 7, 8).

The gene encoding RIBOSOMAL PROTEIN S21 was finally selected as a reference as it shows the least variability out of all candidates tested (Figure 4). Analysis of a large number of genes will be required in the future to identify stably expressed reference genes for nutrient deprivation experiments in unicellular algae.

\subsection{Several LB proteins were identified in this study}

Since LBs are no longer regarded as passive constituents of the cell, an interest in proteins associated with this organelle has arisen and is reflected in an increasing number of publications on the subject. The number and identity of proteins that are found to be associated with LBs in the same alga varies between studies. This becomes obvious when comparing LB proteomes that have been reported for $C$. reinhardtii, the microalga that has been most intensively studied in this regard. Different strains of $C$. reinhardtii were cultivated heterotrophically in the same media for these studies, however in one instance not a single LB protein could be identified 
(Wang et al., 2009), whereas 259 were detected by another group (Moellering and Benning, 2010) and 248 by yet another, including 150 proteins that had not been reported before (Nguyen et al., 2011). In one study, 124 proteins were co-immunoprecipitated with MAJOR LIPID DROPLET PROTEIN (MLDP) that had previously not been identified in LBs of the same strain by the same group (Tsai et al., 2015). These discrepancies underline the prominent effect of sample preparation on the loss of information or the occurrence of false positive detection in the identification of algal LB proteins.

LB associated proteins that have been identified in algae so far include highly abundant structural proteins (Davidi et al., 2012; Lin et al., 2012; Moellering and Benning, 2010; Peled et al., 2011; Sharma et al., 2015; Vieler et al., 2012; Yoneda et al., 2016) and enzymes involved in lipid metabolism as well as lipid trafficking (Moellering and Benning, 2010; Nguyen et al., 2011).

In this study, a large number of proteins was identified in samples derived from $L$. incisa LBs most likely due to the use of highly sensitive liquid chromatography coupled to tandem mass spectrometry (Table 31). The resulting list of putatively LB associated proteins contains a high proportion of proteins that obviously originated from membranes of other organelles contaminating the sample, a common aspect of LB isolation from microalgae (Ding et al., 2012; Nguyen et al., 2011; Nojima et al., 2013; Tzen et al., 1997; Zienkiewicz et al., 2014). Alternatively, the occurrence of these proteins may suggest that organelle membranes indeed interact with LBs in algae.

In addition to contamination from membranes, the LBs isolated by sucrose gradient centrifugation could also be mixed with plastoglobules, carotenoid-enriched smaller structures that derive from the thylakoids. So far they have been described for higher plants and the green alga Dunaliella bardawil (Bréhélin et al., 2007; Katz et al., 1995). The plastoglobule proteome has been analyzed in this alga (Davidi et al., 2015) as well as in $A$. thaliana (Lundquist et al., 2012) and exhibits only very little overlap with the LB proteome in the respective organism. To date plastoglobules have not been observed in electron microscopic analysis of L. incisa (Merzlyak et al., 2007) and a gene encoding a homolog of plastoglobulin, a protein that is characteristic for these structures (Bréhélin et al., 2007), is absent from its genome (data not shown). Therefore it is not likely that the LB protein extracts analyzed in this study was contaminated with plastoglobule proteins. 
5.3.1 LiOGP, g15430 and g13747 are LB associated proteins of unknown function

The screen for $L$. incisa LB associated proteins carried out in this study revealed three proteins that could be verified to localize to these specialized organelles in the tobacco pollen system.

L. incisa OIL GLOBULE PROTEIN (LiOGP) shares homology with a protein that increases in abundance in LB fractions of nitrogen starved Haematococcus pluvialis cells, HpOGP (Peled et al., 2011), but bears no other features that would hint at a possible function (Figure 6). To date, the subcellular localization of the $H$. pluvialis protein has not been confirmed, however its $L$. incisa homolog clearly colocalizes with Nile Red stained neutral lipids in tobacco pollen tubes (Figure 7). LiOGP expression is induced in parallel with the formation of LBs (Figure 8), very much like HpOGP (Peled et al., 2011) and C. reinhardtii MLDP (Moellering and Benning, 2010). In conjunction with the protein's relatively high abundance in LB fractions, this points to an involvement of the protein in LB homeostasis such as the functions that have been elucidated for $A$. thaliana oleosins, the most extensively studied structural LB proteins (Deruyffelaere et al., 2015; Lévesque-Lemay et al., 2015; Shimada et al., 2008; Siloto et al., 2006). In $A$. thaliana seeds, OLEOSIN 1 prevents LBs from coalescing and thus ensures a high surface/volume ratio of the TAG storage organelles (Siloto et al., 2006), a function that is also fulfilled by the major lipid droplet proteins in the microalgae C. reinhardtii (Moellering and Benning, 2010) and Nannochloropsis (Vieler et al., 2012). A comparable role for LiOGP was tested by heterologous seed-specific expression of the algal gene in the A. thatiana oleosin 1 mutant, however an effect on LB size could not be observed in imbibed seeds or early stages of germination (data not shown).

In contrast to LiOGP, for the g15430 protein a close relative could not be identified. The protein is clearly localized at the LBs when expressed in N. tabacum pollen tubes (Figure 9). The domain of unknown function taking up a considerable fraction of the relatively small protein (85 of 287 amino acids, Figure 10) is only present in uncharacterized proteins of a small subset of green algae and might be implicated in a process that is distinct from other algae, however the nature of this function cannot be inferred from the information gained in this study. Expression of the g15430 gene is rapidly modified in response to both media changes during the growth experiment (Figure 11). It appears to take a course independent from TAG accumulation during the first phase, whereas it clearly follows a trend opposite of TAG in the recovery stage. 
While members of the oleosin family are anchored in LBs via a hydrophobic domain that contains a proline knot motif (Tzen et al., 1992) and comprises a large part of the protein, these features cannot be found in LiOGP, g15430 or a range of other proteins for which LB localization has been established (Athenstaedt and Daum, 2003; Eastmond, 2006; Moellering and Benning, 2010). Tandem repeats possibly forming amphipathic helices, which are known to enable perilipins to attach to the LB surface in mammalian cells (Rowe et al., 2016), could not be identified either based on the amino acid sequences using an in silico method as described previously (Rowe et al., 2016, data not shown). It is therefore unclear by which mechanism LiOGP and g15430 attach to LBs.

The g13747 protein is distinguished from the other LB proteins described above in being substantially larger (805 amino acids) and having a striking hydrophobic domain (Figure 13). This feature of the protein spans a part of the amino acid sequence that is even larger than the one described for plant oleosins (approximately 120 amino acid residues compared to approximately 70 residues). The proline knot motif required for anchoring oleosins in LBs (Abell et al., 1997) is not easily discernable in g13747, which is due to the lack of sequence homology with oleosin rather than a shortage of proline residues in the hydrophobic stretch.

Each of the three LB associated proteins of unknown function that were isolated from nitrogen starved L. incisa cells could serve a function analogous to plant oleosins, protecting lipid droplets from premature degradation (Deruyffelaere et al., 2015) and ensuring a high surface/volume ratio to facilitate access for lipases when rapid TAG hydrolysis is required (Siloto et al., 2006).

\subsection{2 g13945 is an LB-localized putative lipase}

The g13945 protein is classified in this study as a putative lipase based on the presence of a partial lipase class 3 domain including a predicted active site (Figure 15). The L. incisa genome contains 53 genes harboring this domain, compared to 38 found in A. thaliana (Li et al., 2012a). This discrepancy reflects the overall differences in genome size and putative number of open reading frames between the two organisms, as in $A$. thaliana the latter is only two thirds of that predicted for L. incisa (data not shown). Since the domain is conserved in a wide range of lipases (Li et al., 2012a) including Ricinus communis acid TAG lipase (Eastmond, 2004), the generally high number of lipase class 3 genes is hardly surprising. This diversity poses a difficulty in the functional annotation of lipases ( $\mathrm{Li}$ et al., 2012a), as recombinant expression 
and activity assays with a wide range of substrates and conditions may be required before the enzymatic activity can be clearly defined.

Irrespective of sequence similarities, structural homology to a lipase of known function can be a useful indicator of the reaction catalyzed by the protein. The predicted three-dimensional structure of g13945 is highly similar to that of a group of fungal and yeast secretory lipases, including Rhizomucor miebei lipase, the first lipase for which a crystal structure could be analyzed and in which a serine-histidine-aspartate catalytic triad was identified in the active site (Brady et al., 1990). Another member of this group, M. globosa LIPASE 1 (MgLIP1) is unusual in exclusively degrading mono- and diacylglycerols in contrast to its relatives that also accept TAG (DeAngelis et al., 2007) and it has been proposed that relatively large hydrophobic residues in the vicinity of catalytic residues cause this distinction (Xu et al., 2012). Protein g13945 can be modeled on the experimentally determined MgLIP1 structure and has no such amino acids in the respective regions that could cause a steric hindrance (Figure 17), TAG is therefore a possible substrate for this putative lipase.

At first sight, the expression pattern of the g13945 gene may point to another direction (Figure 16), as it does not seem plausible for a TAG lipase to be most highly expressed in a stage of the algal culture that is characterized by TAG accumulation. Nevertheless, the protein might be synthesized in inactive form during this stage, ready to be activated as soon as environmental conditions once again permit growth. A similar succession of mechanisms has been proposed for $A$. thaliana SUGAR DEPENDENT 1 (AtSDP1), a lipase that is responsible for bulk TAG degradation during post-germinative growth of $A$. thaliana seedlings (Eastmond, 2006).

Even so, the L. incisa putative lipase was only able to effect a very weak functional complementation of hypocotyl elongation in etiolated seedlings of an $A$. thaliana mutant lacking both SDP1 and its paralog SDP1-L (Figure 18). There is a variety of possible reasons for this: For instance, g13945 protein biosynthesis may be taking place at a slow rate due to differences in codon usage. The algal coding sequence contains $61.7 \%$ guanidine and cytosine nucleotides compared to an average of only approximately $44 \%$ for $A$. thaliana coding sequences (TAIR). Alternatively, posttranslational modifications (PTMs) of the recombinant protein may have differed from the protein modifications taking place in L. incisa, negatively influencing enzyme activity. While phosphorylation sites could not be predicted in the g13945 amino acid sequence using algorithms trained on higher plants (data not shown), other PTMs may be essential for this protein to be active. PTMs in algae analyzed so far do not seem to 
diverge from those in higher plants (Kurotani and Sakurai, 2015), but they have not been extensively studied, so that his explanation cannot be completely ruled out. Independently of these considerations, the enzyme may have a substrate specificity that differs from that of AtSDP1 and AtSDP1-L. TAG in A. thaliana seeds mostly contains 18:1 (n-9), 18:2 (n-6), 18:3 (n-3) and 20:1 (n-9) (Taylor et al., 1995), whereas the strain of L. incisa investigated in this study produces TAG that is especially rich in 18:1 (n-9) and ARA. It is also conceivable that g13945 does not primarily hydrolyze TAG but is rather active on mono- and diacylglycerol (MAG and DAG). The A. thaliana sdp1/sdp1-L mutant retains most of the MAG and DAG lipase activity found in the wildtype (Kelly et al., 2011), therefore the respective enzyme activity would not effect a clear functional complementation in the mutant background.

The lipase assay in which the degradation of ${ }^{14} \mathrm{C}$ labeled triolein was monitored remained equally inconclusive, as the complemented lines behaved similarly as the $s d p 1 / s d p 1-L$ mutant under all conditions and a distinct difference to the wildtype could not be observed at $\mathrm{pH} 7$ or 8 , the $\mathrm{pH}$ optimum for AtSDP1 activity (Eastmond, 2006). Reactions at pH 6 yielded different results, as the wildtype clearly exhibited higher TAG lipase activity than the mutant and complemented lines. This may be caused by one of two possible underlying processes: In $3 \mathrm{~d}$ old wildtype seedlings, the formation of DAG by AtSDP1 and AtSDP1-L may induce formation or activity of DAG and MAG lipases with a lower $\mathrm{pH}$ optimum, catalyzing TAG hydrolysis as a secondary function. Alternatively, gene expression of additional TAG lipases that are most active in slightly alkaline conditions in other cellular compartments may be induced in the mutant background, therefore contributing to TAG degradation in the crude cell extract but not during seedling post-germinative growth. For all plant lines used in this assay, overall TAG lipase activity was highest at $\mathrm{pH} 8$, supporting the latter hypothesis. The lipase activity assay could be refined by using isolated LB membranes (Eastmond, 2006; Kelly et al., 2011) instead of a crude extract, presuming that g13945 localizes to the LBs not only in L. incisa and N. tabacum pollen tubes, but also in germinating $A$. thaliana seedlings.

Semi-quantitative enrichment analysis is a useful way of retrieving proteins that are in fact associated with LBs from complex proteomics data. This was demonstrated by confirming subcellular localization through heterologous expression in tobacco pollen tubes, a valuable system for this type of analysis (Dr. Till Ischebeck, personal communication). The dataset obtained in this study could be helpful in identifying additional LB proteins in L. incisa. 


\section{$5.4 \mathrm{~g} 2905$ is a putative lipase}

The L. incisa g2905 protein has extensive sequence homology with human GASTRIC LIPASE A (HsLIPA, Bodmer et al., 1987) and was therefore identified as a putative TAG lipase (Figure 19). It contains an $\alpha / \beta$ hydrolase domain conserved across a wide range of enzymes (reviewed in Holmquist, 2000), including a catalytic triad typical of serine hydrolases (Brady et al., 1990; Ollis et al., 1992). Many lipases harboring this domain undergo a conformational change at the lipid/water interface termed interfacial activation (Brzozowski et al., 1991). Studies on algal homologs of g2905 are limited to gene expression analyses of C. reinhardtii putative TRIACYLGLYCEROL LIPASE 2 (CrLIP2) in different stress conditions, demonstrating upregulation under nitrogen starvation (Boyle et al., 2012) and downregulation in response to heat stress, during which PUFAs are transferred from membrane lipids to DAG and TAG (Légeret et al., 2016). These findings do not seem to implicate the C. reinhardtii protein in neutral lipid hydrolysis, but the different pattern of g2905 gene expression, reaching a maximum immediately prior to TAG degradation (Figure 20), hints at a possible role in storage lipid mobilization for the L. incisa homolog. This notion is contradicted by the observation that g2905 was not detected in LB protein extracts of nitrogen starved L. incisa cells and did not localize to these organelles in tobacco pollen tubes (Figure 21).

In two different TAG lipase activity assays with heterologous expression of g2905 in the A. thaliana sdp1/sdp1-L mutant, similar results were obtained as for g13945 (Figure 22, see section 5.3.2). A TAG lipase activity could therefore not be determined for this protein despite the sequence homology with a human TAG lipase and the gene expression pattern, which is in line with the fact that it could not be observed to localize at the LBs in L. incisa or in N. tabacum pollen tubes.

\subsection{LiSDP1 is a TAG lipase}

There is another family of lipases that is defined by a common patatin domain, named after a phospholipase A that is highly abundant in potato tubers (Andrews et al., 1988). The family of patatin domain lipases comprises diverse enzymatic activities including the yeast TAG lipases TGL3, 4 and 5 (Athenstaedt and Daum, 2003, 2005) and A. thaliana SDP1 (Eastmond, 2006). The latter is involved in seedling establishment by hydrolyzing the majority of TAG in seedlings in conjunction with SDP1-L. An AtSDP1 homolog has recently been identified in the diatom 
P. tricornutum (Barka et al., 2016) and its ability to hydrolyze the TAG substrate analog para-nitrophenyl butyrate along with an observed TAG accumulation in knockdown mutants point to a TAG lipase activity. In this study, an L. incisa homolog of AtSDP1 was functionally characterized by heterologous expression in the $A$. thaliana $s d p 1 / s d p 1-L$ background and was able to complement postgerminative seedling growth (Figure 26) as well as TAG hydrolysis as demonstrated by a reduced amount of 20:1 (Figure 28), a marker fatty acid of TAG in seeds (Taylor $e t$ al., 1995). The algal lipase did not fully replace the endogenous SDP1 activity, which becomes evident when comparing with the $s d p 1$ - $L$ single knockout mutant (Kelly et al., 2011). For complemented lines with constitutive expression driven by the Cauliflower Mosaic Virus 35 S promoter (Benfey et al., 1989), this is not likely to be caused by a lower level of gene expression, as the $35 \mathrm{~S}$ promoter has been shown to be equivalent to the endogenous A. thaliana SDP1 promoter in complementing the sdp1 mutant (Kelly et al., 2011). Seed specific transgene expression driven by the Brassica napus napA promoter (Ellerström et al., 1996; Soeda et al., 2005) resulted in a less pronounced effect, which might result from transgene expression being limited to the mature seed stage, when seed storage proteins are accumulating, although napA expression has also been shown during germination in Brassica oleracea (Soeda et al., 2005).

A broad substrate specificity of various TAG compositions has been determined for AtSDP1 (Eastmond, 2006) and only minor differences in the abundance of individual fatty acid species were observed in this study (Figures 28 and 29), which could be related to SDP1-L activity or differential degradation of the fatty acids once removed from the glycerol backbone. A similarly broad spectrum of substrates appears to be turned over by the L. incisa homolog, as the relative fatty acid amount in the complemented lines in comparison to the mutant is comparable across all fatty acids analyzed in this experiment. It is however conceivable that the algal enzyme is adapted to the ARA-rich TAG species present in its endogenous environment, resulting in reduced overall activity in the seedling as discussed above.

Some similarities can also be seen in the expression patterns of the two SDP1 genes. At SDP1 is most highly expressed during seed maturation (Eastmond, 2006), which is characterized by intense TAG accumulation (Hills, 2004), pointing to a regulation of enzymatic activity by post-translational mechanisms. LiSDP1 expression undergoes a less drastic increase during the TAG synthesis phase and strongly responds to the onset of growth conditions favoring degradation of the storage lipid (Figure 24). This observation further supports the functional 
similarity of the enzymes, which is substantiated by the subcellular localization of recombinant LiSDP1 in tobacco pollen tubes resembling that of AtSDP1 in A. thaliana seeds.

LiSDP1 is only relatively weakly expressed in L. incis a cells cultivated without nitrogen, which might explain why the protein was not detected in LB extracts in this study. The composition of the LB proteome is likely to undergo dramatic changes in response to nitrogen resupply as demonstrated for C. reinhardtii (Tsai et al., 2015) and it can be assumed that TAG lipases need to be recruited to the sites of neutral lipid storage to access the cell's carbon and energy reserves.

In summary, LiSDP1 is a functional lipase accepting TAG molecules with diverse fatty acid compositions as substrates. It cannot be excluded that it is also capable of hydrolyzing other lipids such as DAG or MAG which were not included in this study, however based on extensive similarity to the homologous $A$. thaliana enzyme such an additional activity seems unlikely.

\subsection{Concluding remarks}

Oleaginous microalgae are a diverse group of organisms in terms of both their natural habitat and their phylogeny. Nonetheless, they share a remarkable trait that draws the attention of cell biologists as well as biotechnologists (Gimpel et al., 2015; Vitova et al., 2015): The accumulation of large quantities of neutral lipids, in some cases rich in PUFAs.

At present, a wide range of molecular biology tools and resources is only available for one species of microalga, C. reinhardtii. This chlorophyte has been very useful in elucidating cellular functions including algal lipid metabolism, however it is not ideally suited to studying the biogenesis of LBs. This is due to the fact that it is not per se an oleaginous organism but rather needs to be stressed by nutrient deprivation or mutated in starch synthesis (Merchant $e t$ al., 2012) in order to permit the observation of considerable LB formation.

In this study, LB biogenesis was analyzed in L. incisa, a strong producer of TAG abundant in ARA. The focus in this thesis was therefore on LB-associated proteins and putative TAG lipases. The data obtained in this work can be a promising resource for the future identification of additional LB proteins in this microalga and comparative proteomics of LBs from different growth stages could provide valuable insight into the dynamics of this organelle. Functional analysis of novel proteins will require refined assays, including a broader range of lipase activity 
assays to accommodate for the variety of substrate specificities and subcellular localizations of these enzymes.

The isolation of relatively pure organelles from this alga would be a very helpful advancement for the identification of LB proteins. This will only be possible if the cells can be easily ruptured, as is the case for the relatively fragile green microalga Dunaliella salina (Davidi et al., 2012) and the C. reinhardtii cell wall mutant $c w-15$ (Davies and Plaskitt, 1971) frequently used for cell fractionation. The isolation of an L. incisa mutant deficient in cell wall synthesis would not only alleviate this obstacle but it could also contribute substantially to the development of more efficient transformation procedures and the establishment of selectable markers for this organism. These are prerequisites for studies of gene function in their endogenous environment by means of knockout, knockdown and overexpression studies. Such transformable strains could also allow L. incisa to be included in the growing group of organisms that can be genetically manipulated with extraordinary precision by means of TALEN or CRISPR technology (Liu et al., 2013), further improving the prospects of studying this unique organism. 


\section{Summary}

Microalgae hold great promise with regards to the production of valuable products such as PUFAs and biofuel. They are a highly interesting group of organisms for investigating lipid metabolism and while some insight has been gained from comparison of C. reinhardtii to other well characterized model organisms, it is becoming increasingly clear that substantial diversity exists between algal species. Among them, the terrestrial green microalga L. incisa is unique in its ability to accumulate high levels of ARA and sequester it in neutral lipids within LBs, especially when deprived of nitrogen.

In order to understand the unique mechanisms of sequestering ARA in neutral lipids, LB biogenesis was analyzed on a protein level in L. incisa strain SAG 2468. Following $3 \mathrm{~d}$ of nitrogen limitation, a state characterized by TAG and ARA accumulation, a multitude of proteins could be identified in LB isolates by means of LC-MS/MS. Semi-quantitative enrichment analysis through comparison with other cellular fractions was carried out and yielded a number of candidate LB associated proteins. For a subset of these candidates, the subcellular localization was confirmed by heterologous expression in tobacco pollen tubes along with confocal microscopy. Additionally, gene expression was analyzed in L. incisa cultures subjected to nitrogen starvation and subsequently rescued by nitrogen resupply, a time course during which TAG is first accumulated and then remobilized.

The proteins g555, g15430 and g13747 were found to be putative structural components of the lipid storage organelle based on similarities to known algal proteins, strong enrichment in the L. incisa LB fraction and hydrophobicity of the amino acid sequence, respectively.

Furthermore, two putative lipases were investigated in this study, one of them LB-associated. Even though TAG lipase activity could not be established for either of them in this study, they may still play a role in L. incisa LB homeostasis.

An additional lipase candidate, LiSDP1, was demonstrated to hydrolyze TAG when the gene was expressed in an $A$. thaliana mutant lacking both plant homologs. The protein appears to localize to LBs in tobacco pollen tubes and is postulated to be involved in the degradation of L. incisa LBs during recovery from nitrogen starvation.

Altogether, this study saw the successful isolation and confirmation of LB proteins from L. incisa as well as the identification of a TAG lipase that is most likely involved in storage lipid degradation, thereby contributing to the elucidation of LB biogenesis in this unique microalga. 


\section{References}

Aaij, C., and Borst, P. (1972). The gel electrophoresis of DNA. BBA Sect. Nucleic Acids Protein Synth. 269, 192-200.

Abell, B.M., Holbrook, L.A., Abenes, M., Murphy, D.J., Hills, M.J., and Moloney, M.M. (1997). Role of the proline knot motif in oleosin endoplasmic reticulum topology and oil body targeting. Plant Cell 9, 1481-1493.

Allen, J.W., Dirusso, C.C., and Black, P.N. (2015). Triacylglycerol synthesis during nitrogen stress involves the prokaryotic lipid synthesis pathway and acyl chain remodeling in the microalgae Coccomyxa subellipsoidea. Algal Res. 10,110-120.

Allen, M.D., Kropat, J., Tottey, S., Del Campo, J.A., and Merchant, S.S. (2007). Manganese deficiency in Chlamydomonas results in loss of photosystem II and MnSOD function, sensitivity to peroxides, and secondary phosphorus and iron deficiency. Plant Physiol. 143, $263-277$.

Altschul, S., Gish, W., Miller, W., Myers, E.W., and Lipman, D.J. (1990). Basic local alignment search tool. J. Mol. Biol. 215, 403-410.

Andrews, D.L., Beames, B., Summers, M.D., and Park, W.D. (1988). Characterization of the lipid acyl hydrolase activity of the major potato (Solanum tuberosum) tuber protein, patatin, by cloning and abundant expression in a baculovirus vector. Biochem J 252, 199-206.

Athenstaedt, K., and Daum, G. (2003). YMR313c/TGL3 encodes a novel triacylglycerol lipase located in lipid particles of Saccharomyces cerevisiae. J. Biol. Chem. 278, 23317-23323.

Athenstaedt, K., and Daum, G. (2005). Tgl4p and Tg15p, two triacylglycerol lipases of the yeast Saccharomyces cerevisiae are localized to lipid particles. J. Biol. Chem. 280, 3730137309.

Athenstaedt, K., Zweytick, D., Jandrositz, A., and Kohlwein, S.D. (1999). Identification and characterization of major lipid particle proteins of the yeast Saccharomyces cerevisiae. J. Bacteriol. 181, 6441-6448.

Bailey, A.P., Koster, G., Guillermier, C., Hirst, E.M.A., MacRae, J.I., Lechene, C.P., Postle, A.D., and Gould, A.P. (2015). Antioxidant role for lipid droplets in a stem cell niche of Drosophila. Cell 163, 340-353.

Barka, F., Angstenberger, M., Ahrendt, T., Lorenzen, W., Bode, H.B., and Büchel, C. (2016). Identification of a triacylglycerol lipase in the diatom Phaeodactylum tricornutum. Biochim. Biophys. Acta - Mol. Cell Biol. Lipids 1861, 239-248.

Bartz, R., Zehmer, J.K., Zhu, M., Chen, Y., Serrero, G., Zhao, Y., and Liu, P. (2007). Dynamic activity of lipid droplets: Protein phosphorylation and GTP-mediated protein translocation. J. Proteome Res. 6, 15-23.

Baud, S., Dichow, N.R., Kelemen, Z., D’Andréa, S., To, A., Berger, N., Canonge, M., 
Kronenberger, J., Viterbo, D., Dubreucq, B., et al. (2009). Regulation of HSD1 in seeds of arabidopsis thaliana. Plant Cell Physiol. 50, 1463-1478.

Beilstein, F., Carrière, V., Leturque, A., and Demignot, S. (2015). Characteristics and functions of lipid droplets and associated proteins in enterocytes. Exp. Cell Res. 340, 172-179.

Beller, M. (2006). Characterization of the Drosophila lipid droplet subproteome. Mol. Cell. Proteomics 5, 1082-1094.

Benfey, P.N., Ren, L., and Chua, N.H. (1989). The CaMV 35S enhancer contains at least two domains which can confer different developmental and tissue-specific expression patterns. EMBO J. 8, 2195-2202.

Bertani, G. (1951). Studies on lysogenesis. I. The mode of phage liberation by lysogenic Escherichia coli. J. Bacteriol. 62, 293-300.

Bigogno, C., Khozin-Goldberg, I., Boussiba, S., Vonshak, A., and Cohen, Z. (2002a). Lipid and fatty acid composition of the green oleaginous alga Parietochloris incisa, the richest plant source of arachidonic acid. Phytochemistry 60, 497-503.

Bigogno, C., Khozin-Goldberg, I., and Cohen, Z. (2002b). Accumulation of arachidonic acidrich triacylglycerols in the microalga Parietochloris incisa (trebuxiophyceae, chlorophyta). Phytochemistry 60, 135-143.

Bigogno, C., Khozin-Goldberg, I., Adlerstein, D., and Cohen, Z. (2002c). Biosynthesis of arachidonic acid in the oleaginous microalga Parietochloris incisa (Chlorophyceae): radiolabeling studies. Lipids 37, 209-216.

Blaby, I.K., Glaesener, A.G., Mettler, T., Fitz-Gibbon, S.T., Gallaher, S.D., Liu, B., Boyle, N.R., Kropat, J., Stitt, M., Johnson, S., et al. (2013). Systems-level analysis of nitrogen starvation-induced modifications of carbon metabolism in a Chlamydomonas reinhardtii starchless mutant. Plant Cell 25, 4305-4323.

Blanc, G., Agarkova, I., Grimwood, J., Kuo, A., Brueggeman, A., Dunigan, D.D., Gurnon, J., Ladunga, I., Lindquist, E., Lucas, S., et al. (2012). The genome of the polar eukaryotic microalga Coccomyxa subellipsoidea reveals traits of cold adaptation. Genome Biol. 13, R39.

Blank, M.L., and Snyder, F. (1975). Quantitative aspects of thin-layer chromatography in the analysis of phosphorus-free lipids. In Analysis of Lipids and Lipoproteins, E.G. Perkins, ed. (Champaign, Illinois: American Oil Chemists' Society), pp. 63-69.

Bodmer, M.W., Angal, S., Yarranton, G.T., Harris, T.J.R., Lyons, A., King, D.J., Pieroni, G., Riviere, C., Verger, R., and Lowe, P.A. (1987). Molecular cloning of a human gastric lipase and expression of the enzyme in yeast. BBA - Gene Struct. Expr. 909, 237-244.

Bosma, M. (2015). Lipid droplet dynamics in skeletal muscle. Exp. Cell Res. 340, 180-186.

Boyle, N.R., Page, M.D., Liu, B., Blaby, I.K., Casero, D., Kropat, J., Cokus, S.J., HongHermesdorf, A., Shaw, J., Karpowicz, S.J., et al. (2012). Three acyltransferases and nitrogenresponsive regulator are implicated in nitrogen starvation-induced triacylglycerol accumulation 
in Chlamydomonas. J. Biol. Chem. 287, 15811-15825.

Bradford, M.M. (1976). A rapid and sensitive method for the quantitation of microgram quantities of protein utilizing the principle of protein-dye binding. Anal. Biochem. 72, 248254.

Brady, L., Brzozowski, A.M., Derewenda, Z.S., Dodson, E., Dodson, G., Tolley, S., Turkenburg, J.P., Christiansen, L., Huge-Jensen, B., Norskov, L., et al. (1990). A serine protease triad forms the catalytic centre of a triacylglycerol lipase. Nature 343, 767-770.

Brasaemle, D.L., Dolios, G., Shapiro, L., and Wang, R. (2004). Proteomic analysis of proteins associated with lipid droplets of basal and lipolytically stimulated 3T3-L1 adipocytes. J. Biol. Chem. 279, 46835-46842.

Bréhélin, C., Kessler, F., and van Wijk, K.J. (2007). Plastoglobules: versatile lipoprotein particles in plastids. Trends Plant Sci. 12, 260-266.

Brzozowski, A.M., Derewenda, U., Derewenda, Z.S., Dodson, G.G., Lawson, D.M., Turkenburg, J.P., Bjorkling, F., Huge-Jensen, B., Patkar, S.A., and Thim, L. (1991). A model for interfacial activation in lipases from the structure of a fungal lipase-inhibitor complex. Nature 351, 491-494.

Carr, R.M., and Ahima, R.S. (2015). Pathophysiology of lipid droplet proteins in liver diseases. Exp. Cell Res. 340, 187-192.

Chapman, K.D., Dyer, J.M., and Mullen, R.T. (2012). Biogenesis and functions of lipid droplets in plants: Thematic Review Series: Lipid Droplet Synthesis and Metabolism: from Yeast to Man. J. Lipid Res. 53, 215-226.

Chen, C.X., Sun, Z., Cao, H.S., Fang, F.L., Ouyang, L.L., and Zhou, Z.G. (2015). Identification and characterization of three genes encoding acyl-CoA: Diacylglycerol acyltransferase (DGAT) from the microalga Myrmecia incisa Reisigl. Algal Res. 12, 280-288.

Chen, E.C., Tai, S.S., Peng, C.C., and Tzen, J.T. (1998). Identification of three novel unique proteins in seed oil bodies of sesame. Plant Cell Physiol. 39, 935-941.

Chen, J.C., Tsai, C.C., and Tzen, J.T. (1999). Cloning and secondary structure analysis of caleosin, a unique calcium-binding protein in oil bodies of plant seeds. Plant Cell Physiol. 40, 1079-1086.

Cheng-Wu, Z., Cohen, Z., Khozin-Goldberg, I., and Richmond, A. (2002). Characterization of growth and arachidonic acid production of Parietochloris incisa comb. nov (Trebouxiophyceae, Chlorophyta). J. Appl. Phycol. 14, 453-460.

Chomcyzynski, P., and Sacchi, N. (1987). Single-step method of RNA isolation by acid guanidinium thiocyanate-. Anal Biochem 162, 156-159.

Clausen, M.K., Christiansen, K., Jensen, P.K., and Behnke, O. (1974). Isolation of lipid particles from baker's yeast. FEBS Lett. 43, 176-179. 
d'Andréa, S., Canonge, M., Beopoulos, A., Jolivet, P., Hartmann, M.A., Miquel, M., Lepiniec, L., and Chardot, T. (2007). At5g50600 encodes a member of the short-chain dehydrogenase reductase superfamily with $11 \beta$ - and $17 \beta$-hydroxysteroid dehydrogenase activities associated with Arabidopsis thaliana seed oil bodies. Biochimie 89, 222-229.

Dahlhoff, M., Fröhlich, T., Arnold, G.J., Müller, U., Leonhardt, H., Zouboulis, C.C., and Schneider, M.R. (2015). Characterization of the sebocyte lipid droplet proteome reveals novel potential regulators of sebaceous lipogenesis. Exp. Cell Res. 332, 146-155.

Dahlqvist, A., Stahl, U., Lenman, M., Banas, A., Lee, M., Sandager, L., Ronne, H., and Stymne, S. (2000). Phospholipid:diacylglycerol acyltransferase: an enzyme that catalyzes the acyl-CoAindependent formation of triacylglycerol in yeast and plants. Proc. Natl. Acad. Sci. U. S. A. 97, 6487-6492.

Daum, G., and Paltauf, F. (1980). Triacylglycerols as fatty acid donors for membrane phospholipid biosynthesis in yeast. Monatshefte Für Chemie 111, 355-363.

Davidi, L., Katz, A., and Pick, U. (2012). Characterization of major lipid droplet proteins from Dunaliella. Planta 236, 19-33.

Davidi, L., Levin, Y., Ben-Dor, S., and Pick, U. (2015). Proteome analysis of cytoplasmatic and plastidic beta-carotene lipid droplets in Dunaliella bardawil. Plant Physiol. 167, 60-79.

Davies, D.R., and Plaskitt, A. (1971). Genetical and structural analyses of cell-wall formation in Chlamydomonas reinhardi. Genet. Res. 17, 33.

Davis, B.J. (1964). Disc electrophoresis. II. Method and application to human serum proteins. Ann. N. Y. Acad. Sci. 121, 404-427.

DeAngelis, Y.M., Saunders, C.W., Johnstone, K.R., Reeder, N.L., Coleman, C.G., Kaczvinsky, J.R., Gale, C., Walter, R., Mekel, M., Lacey, M.P., et al. (2007). Isolation and expression of a Malassezia globosa lipase gene, LIP1. J. Invest. Dermatol. 127, 2138-2146.

Derewenda, Z.S., and Derewenda, U. (1991). Relationships among serine hydrolases: evidence for a common structural motif in triacylglyceride lipases and esterases. Biochem. Cell Biol. 69, 842-851.

Deruyffelaere, C., Bouchez, I., Morin, H., Guillot, A., Miquel, M., Froissard, M., Chardot, T., and D'Andréa, S. (2015). Ubiquitin-mediated proteasomal degradation of oleosins is involved in oil body mobilization during post-germinative seedling growth in Arabidopsis. Plant Cell Physiol. 56, 1374-1387.

Dichlberger, A., Schlager, S., Kovanen, P.T., and Schneider, W.J. (2015). Lipid droplets in activated mast cells - a significant source of triglyceride-derived arachidonic acid for eicosanoid production. Eur. J. Pharmacol.

Ding, Y., Zhang, S., Yang, L., Na, H., Zhang, P., Zhang, H., Wang, Y., Chen, Y., Yu, J., Huo, C., et al. (2012). Isolating lipid droplets from multiple species. Nat. Protoc. 8, 43-51.

Dong, H.-P., Williams, E., Wang, D.-Z., Xie, Z.-X., Hsia, R.-C., Jenck, A., Halden, R., Li, J., 
Chen, F., and Place, A.R. (2013a). Responses of Nannochloropsis oceanica IMET1 to longterm nitrogen starvation and recovery. Plant Physiol. 162, 1110-1126.

Dong, H.-P., Williams, E., Wang, D., Xie, Z.-X., Hsia, R., Jenck, A., Halden, R., Li, J., Chen, F., and Place, A.R. (2013b). Responses of Nannochloropsis oceanica IMET1 to Long-Term Nitrogen Starvation and Recovery. Plant Physiol. 162, 1110-1126.

Doyle, J.J. (1987). A rapid DNA isolation procedure for small quantities of fresh leaf tissue. Phytochem Bull 19, 11-15.

Eastmond, P.J. (2004). Cloning and characterization of the acid lipase from castor beans. J. Biol. Chem. 279, 45540-45545.

Eastmond, P.J. (2006). SUGAR-DEPENDENT1 encodes a patatin domain triacylglycerol lipase that initiates storage oil breakdown in germinating Arabidopsis seeds. Plant Cell 18, $665-675$.

Eichmann, T.O., Grumet, L., Taschler, U., Hartler, J., Heier, C., Woblistin, A., Pajed, L., Kollroser, M., Rechberger, G., Thallinger, G.G., et al. (2015). ATGL and CGI-58 are lipid droplet proteins of the hepatic stellate cell line HSC-T6. J. Lipid Res. 56, 1972-1984.

Ellerström, M., Stålberg, K., Ezcurra, I., and Rask, L. (1996). Functional dissection of a napin gene promoter: identification of promoter elements required for embryo and endospermspecific transcription. Plant Mol. Biol. 32, 1019-1027.

Emanuelsson, O., Nielsen, H., Brunak, S., and von Heijne, G. (2000). Predicting subcellular localization of proteins based on their N-terminal amino acid sequence. J. Mol. Biol. 300, 1005-1016.

Feussner, I., and Kindl, H. (1992). A lipoxygenase is the main lipid body protein in cucumber and soybean cotyledons during the stage of triglyceride mobilization. FEBS Lett. 298, 223225.

Feussner, I., Wasternack, C., Kindl, H., and Kühn, H. (1995). Lipoxygenase-catalyzed oxygenation of storage lipids is implicated in lipid mobilization during germination. Proc. Natl. Acad. Sci. U. S. A. 92, 11849-11853.

Finn, R.D., Bateman, A., Clements, J., Coggill, P., Eberhardt, R.Y., Eddy, S.R., Heger, A., Hetherington, K., Holm, L., Mistry, J., et al. (2014). Pfam: the protein families database. Nucleic Acids Res. 42, D222-D230.

Gimpel, J.A., Henríquez, V., and Mayfield, S.P. (2015). In metabolic engineering of eukaryotic microalgae: Potential and challenges come with great diversity. Front. Microbiol. 6, 1376.

Giroud, C., Gerber, A., and Eichenberger, W. (1988). Lipids of Chlamydomonas reinhardtii Analysis of molecular species and intracellular site(s) of biosynthesis. Plant Cell Physiol. 29, 587-595.

Graham Hope, R., Murphy, D.J., and McLauchlan, J. (2002). The domains required to direct core proteins of hepatitis $\mathrm{C}$ virus and $\mathrm{GB}$ virus-B to lipid droplets share common features 
with plant oleosin proteins. J. Biol. Chem. 277, 4261-4270.

Green, M.R., and Sambrook, J. (2012). Molecular cloning: A laboratory manual (Cold Spring Harbor Laboratory Press).

Greenspan, P., Mayer, E.P., and Fowler, S.D. (1985). Nile red: A selective fluorescent stain for intracellular lipid droplets. J. Cell Biol. 100, 965-973.

Grillitsch, K., Connerth, M., Köfeler, H., Arrey, T.N., Rietschel, B., Wagner, B., Karas, M., and Daum, G. (2011). Lipid particles/droplets of the yeast Saccharomyces cerevisiae revisited: Lipidome meets proteome. Biochim. Biophys. Acta - Mol. Cell Biol. Lipids 1811, 1165-1175.

Grönke, S., Beller, M., Fellert, S., Ramakrishnan, H., Jäckle, H., and Kühnlein, R.P. (2003). Control of fat storage by a Drosophila PAT domain protein. Curr. Biol. 13, 603-606.

Guihéneuf, F., Leu, S., Zarka, A., Khozin-Goldberg, I., Khalilov, I., and Boussiba, S. (2011). Cloning and molecular characterization of a novel acyl-CoA:diacylglycerol acyltransferase 1like gene (PtDGAT1) from the diatom Phaeodactylum tricornutum. FEBS J. 278, 3651-3666.

Hanano, A., Burcklen, M., Flenet, M., Ivancich, A., Louwagie, M., Garin, J., and Blée, E. (2006). Plant seed peroxygenase is an original heme-oxygenase with an EF-hand calcium binding motif. J. Biol. Chem. 281, 33140-33151.

Heilmann, M., Iven, T., Ahmann, K., Hornung, E., Stymne, S., and Feussner, I. (2012). Production of wax esters in plant seed oils by oleosomal cotargeting of biosynthetic enzymes. J. Lipid Res. 53, 2153-2161.

Hills, M.J. (2004). Control of storage-product synthesis in seeds. Curr. Opin. Plant Biol. 7, 302-308.

Hills, M.J., and Murphy, D.J. (1988). Characterization of lipases from the lipid bodies and microsomal membranes of erucic acid-free oilseed-rape (Brassica napus) cotyledons. Biochem. J. 249, 687-693.

Höfgen, R., and Willmitzer, L. (1988). Storage of competent cells for Agrobacterium transformation. Nucleic Acids Res. 16, 9877.

Holmquist, M. (2000). Alpha/beta-hydrolase fold enzymes: structures, functions and mechanisms. Curr. Protein Pept. Sci. 1, 209-235.

Horn, P.J., James, C.N., Gidda, S.K., Kilaru, A., Dyer, J.M., Mullen, R.T., Ohlrogge, J.B., and Chapman, K.D. (2013). Identification of a new class of lipid droplet-associated proteins in plants. Plant Physiol. 162, 1926-1936.

Hornung, E., Krueger, C., Pernstich, C., Gipmans, M., Porzel, A., and Feussner, I. (2005). Production of (10E,12Z)-conjugated linoleic acid in yeast and tobacco seeds. Biochim. Biophys. Acta - Mol. Cell Biol. Lipids 1738, 105-114.

Hu, Q., Sommerfeld, M., Jarvis, E., Ghirardi, M., Posewitz, M., Seibert, M., and Darzins, A. (2008). Microalgal triacylglycerols as feedstocks for biofuel production: Perspectives and 
advances. Plant J. 54, 621-639.

Iskandarov, U., Khozin-Goldberg, I., Ofir, R., and Cohen, Z. (2009). Cloning and characterization of the $\Delta 6$ polyunsaturated fatty acid elongase from the green microalga Parietochloris incisa. Lipids 44, 545-554.

Iskandarov, U., Khozin-Goldberg, I., and Cohen, Z. (2011). Selection of a DGLA-producing mutant of the microalga Parietochloris incisa: I. Identification of mutation site and expression of VLC-PUFA biosynthesis genes. Appl. Microbiol. Biotechnol. 90, 249-256.

Iskandarov, U., Sitnik, S., Shtaida, N., Didi-Cohen, S., Leu, S., Khozin-Goldberg, I., Cohen, Z., and Boussiba, S. (2015). Cloning and characterization of a GPAT-like gene from the microalga Lobosphaera incisa (Trebouxiophyceae): overexpression in Chlamydomonas reinhardtii enhances TAG production. J. Appl. Phycol.

Jacquier, N., Mishra, S., Choudhary, V., and Schneiter, R. (2013). Expression of oleosin and perilipins in yeast promotes formation of lipid droplets from the endoplasmic reticulum. J. Cell Sci. 126, 5198-5209.

Jolivet, P., Roux, E., D’Andrea, S., Davanture, M., Negroni, L., Zivy, M., and Chardot, T. (2004). Protein composition of oil bodies in Arabidopsis thaliana ecotype WS. Plant Physiol. Biochem. 42, 501-509.

Jolivet, P., Boulard, C., Bellamy, A., Larré, C., Barre, M., Rogniaux, H., D’Andréa, S., Chardot, T., and Nesi, N. (2009). Protein composition of oil bodies from mature Brassica napus seeds. Proteomics 9, 3268-3284.

Jolivet, P., Acevedo, F., Boulard, C., d'Andréa, S., Faure, J.-D., Kohli, A., Nesi, N., Valot, B., and Chardot, T. (2013). Crop seed oil bodies: From challenges in protein identification to an emerging picture of the oil body proteome. Proteomics 13, 1836-1849.

Kajikawa, M., Sawaragi, Y., Shinkawa, H., Yamano, T., Ando, A., Kato, M., Hirono, M., Sato, N., and Fukuzawa, H. (2015). Algal dual-specificity tyrosine phosphorylation-regulated kinase, Triacylglycerol Accumulation Regulator1, regulates accumulation of triacylglycerol in nitrogen or sulfur deficiency. Plant Physiol. 168, 752-764.

Karsten, U., Friedl, T., Schumann, R., Hoyer, K., and Lembcke, S. (2005). Mycosporine-like amino acids and phylogenies in green algae: Prasiola and its relatives from the Trebouxiophyceae (Chlorophyta). J. Phycol. 41, 557-566.

Katavic, V., Agrawal, G.K., Hajduch, M., Harris, S.L., and Thelen, J.J. (2006). Protein and lipid composition analysis of oil bodies from two Brassica napus cultivars. Proteomics 6, 45864598.

Katz, A., Jimenez, C., and Pick, U. (1995). Isolation and characterization of a protein associated with carotene globules in the alga Dunaliella bardawil. Plant Physiol. 108, 16571664.

Kellenberger, E. (1968). The Components of the T4 capsid and of other, capsid-related structures. Virology 34, 549-561. 
Kelly, A.A., Quettier, A.-L., Shaw, E., and Eastmond, P.J. (2011). Seed storage oil mobilization is important but not essential for germination or seedling establishment in Arabidopsis. Plant Physiol. 157, 866-875.

Kelly, A.A., van Erp, H., Quettier, A.-L., Shaw, E., Menard, G., Kurup, S., and Eastmond, P.J. (2013). The SUGAR-DEPENDENT1 lipase limits triacylglycerol accumulation in vegetative tissues of Arabidopsis. Plant Physiol. 162, 1282-1289.

Khozin-Goldberg, I., Bigogno, C., Shrestha, P., and Cohen, Z. (2002). Nitrogen starvation induces the accumulation of arachidonic acid in the freshwater green alga Parietochloris incisa (Trebuxiophyceae). J. Phycol. 38, 991-994.

Khozin-Goldberg, I., Shrestha, P., and Cohen, Z. (2005). Mobilization of arachidonyl moieties from triacylglycerols into chloroplastic lipids following recovery from nitrogen starvation of the microalga Parietochloris incisa. Biochim. Biophys. Acta - Mol. Cell Biol. Lipids 1738, 6371.

Khozin-Goldberg, I., Iskandarov, U., and Cohen, Z. (2011). LC-PUFA from photosynthetic microalgae: occurrence, biosynthesis, and prospects in biotechnology. Appl. Microbiol. Biotechnol. 91, 905-915.

Kienesberger, P.C., Oberer, M., Lass, A., and Zechner, R. (2009). Mammalian patatin domain containing proteins: a family with diverse lipolytic activities involved in multiple biological functions. J. Lipid Res. 50 Suppl, S63-S68.

Kim, H.U., Hsieh, K., Ratnayake, C., and Huang, A.H.C. (2002). A novel group of oleosins is present inside the pollen of Arabidopsis. J. Biol. Chem. 277, 22677-22684.

Kozera, B., and Rapacz, M. (2013). Reference genes in real-time PCR. J. Appl. Genet. 54, 391-406.

Kurotani, A., and Sakurai, T. (2015). In Silico analysis of correlations between protein disorder and post-translational modifications in algae. Int. J. Mol. Sci. 16, 19812-19835.

Laemmli, U.K. (1970). Cleavage of structural proteins during the assembly of the head of bacteriophage T4. Nature 227, 680-685.

Layerenza, J.P., González, P., García De Bravo, M.M., Polo, M.P., Sisti, M.S., and Ves-Losada, A. (2013). Nuclear lipid droplets: A novel nuclear domain. Biochim. Biophys. Acta - Mol. Cell Biol. Lipids 1831, 327-340.

Légeret, B., Schulz-Raffelt, M., Nguyen, H.M., Auroy, P., Beisson, F., Peltier, G., Blanc, G., and Li-Beisson, Y. (2016). Lipidomic and transcriptomic analyses of Chlamydomonas reinhardtii under heat stress unveil a direct route for the conversion of membrane lipids into storage lipids. Plant, Cell Environ. 39, 834-847.

Lemieux, B., Miquel, M., and Somerville, C. (1990). Mutants of Arabidopsis with alterations in seed lipid fatty acid composition. Theor. Appl. Genet. 80, 234-240.

Lévesque-Lemay, M., Chabot, D., Hubbard, K., Chan, J.K., Miller, S., and Robert, L.S. (2015). 
Tapetal oleosins play an essential role in tapetosome formation and protein relocation to the pollen coat.

Li, F., Asami, T., Wu, X., Tsang, E.W.T., and Cutler, A.J. (2007). A putative hydroxysteroid dehydrogenase involved in regulating plant growth and development. Plant Physiol. 145, 8797.

Li, J., Han, D., Wang, D., Ning, K., Jia, J., Wei, L., Jing, X., Huang, S., Chen, J., Li, Y., et al. (2014). Choreography of transcriptomes and lipidomes of Nannochloropsis reveals the mechanisms of oil synthesis in microalgae. Plant Cell 26, 1645-1665.

Li, W., Ling, H., Zhang, F., Yao, H., Sun, X., and Tang, K. (2012a). Analysis of Arabidopsis genes encoding putative class III lipases. J. Plant Biochem. Biotechnol. 21, 261-267.

Li, X., Benning, C., and Kuo, M.-H. (2012b). Rapid triacylglycerol turnover in Chlamydomonas reinhardtii requires a lipase with broad substrate specificity. Eukaryot. Cell $11,1451-1462$.

Lin, I.P., Jiang, P.L., Chen, C.S., and Tzen, J.T.C. (2012). A unique caleosin serving as the major integral protein in oil bodies isolated from Chlorella sp. cells cultured with limited nitrogen. Plant Physiol. Biochem. 61, 80-87.

Lin, L.-J., Tai, S.S.K., Peng, C.-C., and Tzen, J.T.C. (2002). Steroleosin, a sterol-binding dehydrogenase in seed oil bodies. Plant Physiol. 128, 1200-1211.

Liu, B., and Benning, C. (2013). Lipid metabolism in microalgae distinguishes itself. Curr. Opin. Biotechnol. 24, 300-309.

Liu, W., Yuan, J.S., and Stewart, C.N. (2013). Advanced genetic tools for plant biotechnology. Nat. Rev. Genet. 14, 781-793.

Løvdal, T., and Lillo, C. (2009). Reference gene selection for quantitative real-time PCR normalization in tomato subjected to nitrogen, cold, and light stress. Anal. Biochem. 387, $238-242$.

Love, J.D., Suzuki, T., Robinson, D.B., Harris, C.M., Johnson, J.E., Mohler, P.J., Jerome, W.G., and Swift, L.L. (2015). Microsomal triglyceride transfer protein (MTP) associates with cytosolic lipid droplets in 3T3-L1 adipocytes. PLoS One 10, 1-18.

Lundquist, P.K., Poliakov, A., Bhuiyan, N.H., Zybailov, B., Sun, Q., and van Wijk, K.J. (2012). The functional network of the Arabidopsis plastoglobule proteome based on quantitative proteomics and genome-wide coexpression analysis. Plant Physiol. 158, 1172-1192.

Mähs, A., Steinhorst, L., Han, J.-P., Shen, L.-K., Wang, Y., and Kudla, J. (2013). The calcineurin B-like Ca2+ sensors CBL1 and CBL9 function in pollen germination and pollen tube growth in Arabidopsis. Mol. Plant 6, 1149-1162.

Markham, N.R., and Zuker, M. (2008). UNAFold: software for nucleic acid folding and hybridization. 453 . 
Martin, G.J.O., Hill, D.R.A., Olmstead, I.L.D., Bergamin, A., Shears, M.J., Dias, D.A., Kentish, S.E., Scales, P.J., Botté, C.Y., and Callahan, D.L. (2014). Lipid profile remodeling in response to nitrogen deprivation in the microalgae Chlorella sp. (Trebouxiophyceae) and Nannochloropsis sp. (Eustigmatophyceae). PLoS One 9, e103389.

Martinez-Botas, J., Anderson, J.B., Tessier, D., Lapillonne, A., Chang, B.H., Quast, M.J., Gorenstein, D., Chen, K.H., and Chan, L. (2000). Absence of perilipin results in leanness and reverses obesity in Lepr(db/db) mice. Nat. Genet. 26, 474-479.

Matyash, V., Liebisch, G., Kurzchalia, T. V, Shevchenko, A., and Schwudke, D. (2008). Lipid extraction by methyl-tert-butyl ether for high-throughput lipidomics. J. Lipid Res. 49, 11371146.

May, C., Höhne, M., Gnau, P., Schwennesen, K., and Kindl, H. (2000). The N-terminal $\beta$ barrel structure of lipid body lipoxygenase mediates its binding to liposomes and lipid bodies. Eur. J. Biochem. 267, 1100-1109.

Melo, R.C.N., and Weller, P.F. (2015). Lipid droplets in leukocytes: Organelles linked to inflammatory responses. Exp. Cell Res. 340, 193-197.

Merchant, S.S., Kropat, J., Liu, B., Shaw, J., and Warakanont, J. (2012). TAG, You're it! Chlamydomonas as a reference organism for understanding algal triacylglycerol accumulation. Curr. Opin. Biotechnol. 23, 352-363.

Merzlyak, M.N., Chivkunova, O.B., Gorelova, O.A., Reshetnikova, I. V., Solovchenko, A.E., Khozin-Goldberg, I., and Cohen, Z. (2007). Effect of nitrogen starvation on optical properties, pigments, and arachidonic acid content of the unicellular green alga Parietochloris incisa (Trebouxiophyceae, Chlorophyta). J. Phycol. 43, 833-843.

Meyer, T.S., and Lamberts, B.L. (1965). Use of coomassie brilliant blue R250 for the electrophoresis of microgram quantities of parotid saliva proteins on acrylamide-gel strips. Biochim. Biophys. Acta 107, 144-145.

Miller, R., Wu, G., Deshpande, R.R., Vieler, A., Gärtner, K., Li, X., Moellering, E.R., Zäuner, S., Cornish, A.J., Liu, B., et al. (2010). Changes in transcript abundance in Chlamydomonas reinhardtii following nitrogen deprivation predict diversion of metabolism. Plant Physiol. 154, 1737-1752.

Miquel, M., and Browse, J. (1992). Arabidopsis mutants deficient in polyunsaturated fatty acid synthesis. Biochemical and genetic characterization of a plant oleoyl-phosphatidylcholine desaturase. J. Biol. Chem. 267, 1502-1509.

Mishra, S., and Schneiter, R. (2015). Expression of perilipin 5 promotes lipid droplet formation in yeast. Commun. Integr. Biol. 0889.

Moellering, E.R., and Benning, C. (2010). RNA interference silencing of a major lipid droplet protein affects lipid droplet size in Chlamydomonas reinhardtii. Eukaryot. Cell 9, 97-106.

Moellering, E.R., Miller, R., and Benning, C. (2009). Molecular genetics of lipid metabolism in the model green alga Chlamydomonas reinhardtii. In Lipids in Photosynthesis, pp. 139-155. 
Msanne, J., Xu, D., Konda, A.R., Casas-Mollano, J.A., Awada, T., Cahoon, E.B., and Cerutti, H. (2012). Metabolic and gene expression changes triggered by nitrogen deprivation in the photoautotrophically grown microalgae Chlamydomonas reinhardtii and Coccomyxa sp. C169. Phytochemistry $75,50-59$.

Mullis, K. (1987). Process for amplifying nucleic acid sequences.

Murashige, T., and Skoog, F. (1962). A revised medium for rapid growth and bio assays with tobacco tissue cultures. Physiol. Plant. 15, 473-497.

Murphy, D.J. (2001). The biogenesis and functions of lipid bodies in animals, plants and microorganisms. Prog. Lipid Res. 40, 325-438.

Najt, C.P., Lwande, J.S., Mcintosh, A.L., Senthivinayagam, S., Gupta, S., Kuhn, L.A., and Atshaves, B.P. (2014). Structural and functional assessment of perilipin 2 lipid binding domain(s). Biochemistry 53, 7051-7066.

Neuhoff, V., Arold, N., Taube, D., and Ehrhardt, W. (1988). Improved staining of proteins in polyacrylamide gels including isoelectric focusing gels with clear background at nanogram sensitivity using Coomassie Brilliant Blue G-250 and R-250. Electrophoresis 9, 255-262.

Nguyen, H.M., Baudet, M., Cuiné, S., Adriano, J.-M., Barthe, D., Billon, E., Bruley, C., Beisson, F., Peltier, G., Ferro, M., et al. (2011). Proteomic profiling of oil bodies isolated from the unicellular green microalga Chlamydomonas reinhardtii: With focus on proteins involved in lipid metabolism. Proteomics 11, 4266-4273.

Nichols, B.W., and Appleby, R.S. (1969). The distribution and biosynthesis of arachidonic acid in algae. Phytochemistry 8,1907-1915.

Nojima, D., Yoshino, T., Maeda, Y., Tanaka, M., Nemoto, M., and Tanaka, T. (2013). Proteomics analysis of oil body-associated proteins in the oleaginous diatom. J. Proteome Res. 12, 5293-5301.

Oelkers, P., Cromley, D., Padamsee, M., Billheimer, J.T., and Sturley, S.L. (2002). The DGA1 gene determines a second triglyceride synthetic pathway in yeast. J. Biol. Chem. 277, 88778881.

Ollis, D.L., Cheah, E., Cygler, M., Dijkstra, B., Frolow, F., Franken, S.M., Harel, M., Remington, S.J., Silman, I., and Schrag, J. (1992). The alpha/beta hydrolase fold. Protein Eng. 5, 197-211.

Oñate-Sánchez, L., and Vicente-Carbajosa, J. (2008). DNA-free RNA isolation protocols for Arabidopsis thaliana, including seeds and siliques. BMC Res. Notes 1, 93.

Ornstein, L. (1964). Disc electrophoresis - I Background and theory. Ann. N. Y. Acad. Sci. $121,321-349$.

Ouyang, L., Du, D., Yu, S., Li, C., Zhang, C., Gao, H., and Zhou, Z. (2012). Expressed sequence tags analysis revealing the taxonomic position and fatty acid biosynthesis in an oleaginous green microalga, Myrmecia incisa Reisigl (Trebouxiophyceae, Chlorophyta). 
Chinese Sci. Bull. 57, 3342-3352.

Ouyang, L.-L., Chen, S.-H., Li, Y., and Zhou, Z.-G. (2013). Transcriptome analysis reveals unique C4-like photosynthesis and oil body formation in an arachidonic acid-rich microalga Myrmecia incisa Reisigl H4301. BMC Genomics 14, 396.

Pasaribu, B., Lin, I.P., Chen, C.S., Lu, C.Y., and Jiang, P.L. (2014). Nutrient limitation in Auxenochlorella protothecoides induces qualitative changes of fatty acid and expression of caleosin as a membrane protein associated with oil bodies. Biotechnol. Lett. 36, 175-180.

Pautov, A., Yakovleva, O., Krylova, E., and Gussarova, G. (2016). Large lipid droplets in leaf epidermis of angiosperms. Flora - Morphol. Distrib. Funct. Ecol. Plants.

Peled, E., Leu, S., Zarka, A., Weiss, M., Pick, U., Khozin-Goldberg, I., and Boussiba, S. (2011). Isolation of a novel oil globule protein from the green alga Haematococcus pluvialis (Chlorophyceae). Lipids 46, 851-861.

Powell, D.W., Weaver, C.M., Jennings, J.L., McAfee, K.J., He, Y., Weil, P.A., and Link, A.J. (2004). Cluster analysis of mass spectrometry data reveals a novel component of SAGA. Mol. Cell. Biol. 24, 7249-7259.

Poxleitner, M., Rogers, S.W., Lacey Samuels, A., Browse, J., and Rogers, J.C. (2006). A role for caleosin in degradation of oil-body storage lipid during seed germination. Plant J. 47, 917-933.

Rasband, W.S. ImageJ (Bethesda, Maryland, USA).

Read, S.M., Clarke, A.E., and Bacic, A. (1993). Stimulation of growth of cultured Nicotiana tabacum W 38 pollen tubes by poly(ethylene glycol) and $\mathrm{Cu}(\mathrm{II})$ salts. Protoplasma 177, 1-14.

Reisigl, H. (1964). Zur Systematik und Ökologie alpiner Bodenalgen. Österreichische Bot. Zeitschrift 111, 402-499.

Robinson, D.G., and Schlösser, U.G. (1978). Crystal structure of human gastric lipase and model of lysosomal acid lipase, two lipolytic enzymes of medical interest. J. Biol. Chem. 141, 83-92.

Rowe, E.R., Mimmack, M.L., Barbosa, A.D., Haider, A., Isaac, I., Ouberai, M.M., Thiam, A.R., Patel, S., Saudek, V., Siniossoglou, S., et al. (2016). Conserved amphipathic helices mediate lipid droplet targeting of perilipins 1-3. J. Biol. Chem. M115.691048 - .

Rudolph, M., Schlereth, a., Korner, M., Feussner, K., Berndt, E., Melzer, M., Hornung, E., and Feussner, I. (2011). The lipoxygenase-dependent oxygenation of lipid body membranes is promoted by a patatin-type phospholipase in cucumber cotyledons. J. Exp. Bot. 62, 749-760.

Schmollinger, S., Mühlhaus, T., Boyle, N.R., Blaby, I.K., Casero, D., Mettler, T., Moseley, J.L., Kropat, J., Sommer, F., Strenkert, D., et al. (2014). Nitrogen-sparing mechanisms in Chlamydomonas affect the transcriptome, the proteome, and photosynthetic metabolism. Plant Cell 26, 1410-1435.

Schweiger, M., and Zechner, R. (2015). Breaking the barrier - Chaperone-mediated autophagy 
of perilipins regulates the lipolytic degradation of fat. Cell Metab. 22, 60-61.

Segrest, J.P., Jackson, R.L., Morrisett, J.D., and Gotto, A.M. (1974). A molecular theory of lipid-protein interactions in the plasma lipoproteins. FEBS Lett. 38, 247-258.

Sharma, K.K., Li, Y., and Schenk, P.M. (2015). Rapid lipid induction in Chlorella sp by UV-C radiation. BioEnergy Res. 8, 1824-1830.

Shevchenko, A., Tomas, H., Havliš, J., Olsen, J. V, and Mann, M. (2007). In-gel digestion for mass spectrometric characterization of proteins and proteomes. Nat. Protoc. 1, 2856-2860.

Shi, S., Valle-Rodríguez, J.O., Siewers, V., and Nielsen, J. (2011). Prospects for microbial biodiesel production. Biotechnol. J. 6, 277-285.

Shimada, T.L., and Hara-Nishimura, I. (2015). Leaf oil bodies are subcellular factories producing antifungal oxylipins. Curr. Opin. Plant Biol. 25, 145-150.

Shimada, T.L., Shimada, T., Takahashi, H., Fukao, Y., and Hara-Nishimura, I. (2008). A novel role for oleosins in freezing tolerance of oilseeds in Arabidopsis thaliana. Plant J. 55, 798-809.

Siloto, R.M.P., Findlay, K., Lopez-Villalobos, A., Yeung, E.C., Nykiforuk, C.L., and Moloney, M.M. (2006). The accumulation of oleosins determines the size of seed oilbodies in Arabidopsis. Plant Cell 18,1961-1974.

Simionato, D., Block, M.A., La Rocca, N., Jouhet, J., Maréchal, E., Finazzi, G., and Morosinotto, T. (2013). The response of Nannochloropsis gaditana to nitrogen starvation includes de novo biosynthesis of triacylglycerols, a decrease of chloroplast galactolipids, and reorganization of the photosynthetic apparatus. Eukaryot. Cell 12, 665-676.

Soeda, Y., Konings, M.C.J.M., Vorst, O., van Houwelingen, A.M.M.L., Stoopen, G.M., Maliepaard, C.A., Kodde, J., Bino, R.J., Groot, S.P.C., and van der Geest, A.H.M. (2005). Gene expression programs during Brassica oleracea seed maturation, osmopriming, and germination are indicators of progression of the germination process and the stress tolerance level. Plant Physiol. 137, 354-368.

Solovchenko, A.E., Khozin-Goldberg, I., Didi-Cohen, S., Cohen, Z., and Merzlyak, M.N. (2008a). Effects of light and nitrogen starvation on the content and composition of carotenoids of the green microalga Parietochloris incisa. Russ. J. Plant Physiol. 55, 455-462.

Solovchenko, A.E., Khozin-Goldberg, I., Didi-Cohen, S., Cohen, Z., and Merzlyak, M.N. (2008b). Effects of light intensity and nitrogen starvation on growth, total fatty acids and arachidonic acid in the green microalga Parietochloris incisa. J. Appl. Phycol. 20, 245-251.

Solovchenko, A.E., Khozin-Goldberg, I., Cohen, Z., and Merzlyak, M.N. (2009). Carotenoidto-chlorophyll ratio as a proxy for assay of total fatty acids and arachidonic acid content in the green microalga Parietochloris incisa. J. Appl. Phycol. 21, 361-366.

Sorger, D., and Daum, G. (2002). Synthesis of triacylglycerols by the acyl-coenzyme A : diacyl glycerol acyltransferase Dga1p in lipid particles of the yeast Saccharomyces cerevisiae. J.

Bacteriol. 184, 519-524. 
Stahl, U., Carlsson, A., Lenman, M., Dahlqvist, A., Huang, B., Banás, W., Banás, A., and Stymne, S. (2004). Cloning and functional characterization of a phospholipid : diacylglycerol acyltransferase from Arabidopsis. Am. Soc. Plant Biol. 135, 1324-1335.

Stanier, R.Y., Kunisawa, R., Mandel, M., and Cohen-Bazire, G. (1971). Purification and properties of unicellular blue-green algae (order Chroococcales). Bacteriol. Rev. 35, 171-205.

Sturm, A., Schwennesen, K., and Kindl, H. (1985). Isolation of proteins assembled in lipid body membranes during fat mobilization in cucumber cotyledons. Eur. J. Biochem. 150, 461468.

Sztalryd, C., and Kimmel, A.R. (2014). Perilipins: Lipid droplet coat proteins adapted for tissue-specific energy storage and utilization, and lipid cytoprotection. Biochimie 96, 96-101.

Takahashi, S., Katagiri, T., Yamaguchi-Shinozaki, K., and Shinozaki, K. (2000). An Arabidopsis gene encoding a $\mathrm{Ca} 2+$-binding protein is induced by abscisic acid during dehydration. Plant Cell Physiol. 41, 898-903.

Tardif, M., Atteia, A., Specht, M., Cogne, G., Rolland, N., Brugiere, S., Hippler, M., Ferro, M., Bruley, C., Peltier, G., et al. (2012). PredAlgo: A new subcellular localization prediction tool dedicated to green algae. Mol. Biol. Evol. 29, 3625-3639.

Taylor, D.C., Giblin, E.M., Reed, D.W., and Hogge, L.R. (1995). Stereospecific analysis and mass spectrometry of triacylglycerols from Arabidopsis thaliana (L.) heynh. Columbia seed. J. Am. Oil Chem. Soc. 72, 305-308.

Teixeira, L., Rabouille, C., Rørth, P., Ephrussi, A., and Vanzo, N.F. (2003). Drosophila Perilipin/ADRP homologue Lsd2 regulates lipid metabolism. Mech. Dev. 120, 1071-1081.

Thakur, A., and Bhatla, S.C. (2016). Proteomic analysis of oil body membrane proteins accompanying the onset of desiccation phase during sunflower seed development. Plant Signal. Behav.

Thorne, H. (1967). Electrophoretic characterization and fractionation of polyoma virus DNA. J. Mol. Biol. 24, 203-211.

Tnani, H., López, I., Jouenne, T., and Vicient, C.M. (2011). Protein composition analysis of oil bodies from maize embryos during germination. J. Plant Physiol. 168, 510-513.

Tourasse, N.J., Barbi, T., Waterhouse, J.C., Shtaida, N., Leu, S., Boussiba, S., Purton, S., and Vallon, O. (2014). The complete sequence of the chloroplast genome of the green microalga Lobosphaera (Parietochloris) incisa. Mitochondrial DNA Part A.

Tourasse, N.J., Shtaida, N., Khozin-Goldberg, I., Boussiba, S., and Vallon, O. (2015). The complete mitochondrial genome sequence of the green microalga Lobosphaera (Parietochloris) incisa reveals a new type of palindromic repetitive repeat. BMC Genomics 16, 580 .

Tsai, C.-H., Warakanont, J., Takeuchi, T., Sears, B.B., Moellering, E.R., and Benning, C. (2014). The protein Compromised Hydrolysis of Triacylglycerols 7 (CHT7) acts as a repressor 
of cellular quiescence in Chlamydomonas. Proc. Natl. Acad. Sci. 111, 15833-15838.

Tsai, C.-H., Zienkiewicz, K., Amstutz, C.L., Brink, B.G., Warakanont, J., Roston, R., and Benning, C. (2015). Dynamics of protein and polar lipid recruitment during lipid droplet assembly in Chlamydomonas reinhardtii. Plant J. 83, 650-660.

Twell, D., Wing, R., Yamaguchi, J., and McCormick, S. (1989). Isolation and expression of an anther-specific gene from tomato. MGG Mol. Gen. Genet. 217, 240-245.

Tzen, J.T., Peng, C.C., Cheng, D.J., Chen, E.C., and Chiu, J.M. (1997). A new method for seed oil body purification and examination of oil body integrity following germination. J. Biochem. $121,762-768$.

Tzen, J.T.C., Lie, G.C., and Huang, A.H.C. (1992). Characterization of the charged components and their topology on the surface of plant seed oil bodies. J. Biol. Chem. 267, $15626-15634$.

Untergasser, A., Cutcutache, I., Koressaar, T., Ye, J., Faircloth, B.C., Remm, M., and Rozen, S.G. (2012). Primer3-new capabilities and interfaces. Nucleic Acids Res. 40, 1-12.

Uzbekov, R., and Roingeard, P. (2013). Nuclear lipid droplets identified by electron microscopy of serial sections. BMC Res. Notes 6, 386.

Valledor, L., and Weckwerth, W. (2014). An improved detergent-compatible gel-fractionation LC-LTQ-Orbitrap-MS workflow for plant and microbial proteomics. In Plant Proteomics: Methods and Protocols, J. V. Jorrin-Novo, S. Komatsu, W. Weckwerth, and S. Wienkoop, eds. pp. 85-91.

Vance, V.B., and Huang, A.H. (1987). The major protein from lipid bodies of maize. Characterization and structure based on cDNA cloning. J. Biol. Chem. 262, 11275-11279.

Vermachova, M., Purkrtova, Z., Santrucek, J., Jolivet, P., Chardot, T., and Kodicek, M. (2011). New protein isoforms identified within Arabidopsis thaliana seed oil bodies combining chymotrypsin/trypsin digestion and peptide fragmentation analysis. Proteomics 11, 3430_ 3434.

Vieler, A., Brubaker, S.B., Vick, B., and Benning, C. (2012). A lipid droplet protein of Nannochloropsis with functions partially analogous to plant oleosins. Plant Physiol. 158, 1562-1569.

Vitova, M., Bisova, K., Kawano, S., and Zachleder, V. (2015). Accumulation of energy reserves in algae: From cell cycles to biotechnological applications. Biotechnol. Adv. 33, 1204 1218.

Wang, H., Bell, M., Sreenevasan, U., Hu, H., Liu, J., Dalen, K., Londos, C., Yamaguchi, T., Rizzo, M.A., Coleman, R., et al. (2011). Unique regulation of adipose triglyceride lipase (ATGL) by perilipin 5, a lipid droplet-associated protein. J. Biol. Chem. 286, 15707-15715.

Wang, L., Wang, Y., Liang, Y., Li, J., Liu, Y., Zhang, J., Zhang, A., Fu, J., and Jiang, G. (2013). Specific accumulation of lipid droplets in hepatocyte nuclei of PFOA-exposed BALB/c mice. 
Sci. Rep. 3, 2174.

Wang, W., Wei, S., Li, L., Su, X., Du, C., Li, F., Geng, B., Liu, P., and Xu, G. (2015).

Proteomic analysis of murine testes lipid droplets. Sci. Rep. 5, 12070.

Wang, Z.T., Ullrich, N., Joo, S., Waffenschmidt, S., and Goodenough, U. (2009). Algal lipid bodies: Stress induction, purification, and biochemical characterization in wild-type and starchless chlamydomonas reinhardtit. Eukaryot. Cell 8, 1856-1868.

Watanabe, S., Hirabayashii, S., Boussiba, S., Cohen, Z., Vonshak, A., and Richmond, A. (1996). Parietochloris incisa comb. nov. (Trebouxiophyceae, Chlorophyta). Phycol. Res. 44, 107-108.

Weigel, D., and Glazebrook, J. (2002). Arabidopsis: A laboratory manual (Cold Spring Harbor Laboratory Press).

Wellburn, A.R. (1994). The spectral determination of chlorophylls a and b, as well as total carotenoids, using various solvents with spectrophotometers of different resolution. J. Plant Physiol. 144, 307-313.

Wilfling, F., Wang, H., Haas, J.T., Krahmer, N., Gould, T.J., Uchida, A., Cheng, J.-X., Graham, M., Christiano, R., Fröhlich, F., et al. (2013). Triacylglycerol synthesis enzymes mediate lipid droplet growth by relocalizing from the ER to lipid droplets. Dev. Cell 24, 384 399.

Xin, L., Hong-ying, H., Ke, G., and Ying-xue, S. (2010). Effects of different nitrogen and phosphorus concentrations on the growth, nutrient uptake, and lipid accumulation of a freshwater microalga Scenedesmus sp. Bioresour. Technol. 101, 5494-5500.

Xu, T., Liu, L., Hou, S., Xu, J., Yang, B., Wang, Y., and Liu, J. (2012). Crystal structure of a mono- and diacylglycerol lipase from Malassezia globosa reveals a novel lid conformation and insights into the substrate specificity. J. Struct. Biol. 178, 363-369.

Yatsu, L.Y., and Jacks, T.J. (1972). Spherosome membranes: half unit-membranes. Plant Physiol 49, 937-943.

Yatsu, L.Y., Jacks, T.J., and Hensarling, T.P. (1971). Isolation of spherosomes (oleosomes) from onion, cabbage, and cottonseed tissues. Plant Physiol. 48, 675-682.

Yoneda, K., Yoshida, M., Suzuki, I., and Watanabe, M.M. (2016). Identification of a major lipid droplet protein in a marine diatom Phaeodactylum tricornutum. Plant Cell Physiol. pcr204.

Yoon, K., Han, D., Li, Y., Sommerfeld, M., and Hu, Q. (2012). Phospholipid:diacylglycerol acyltransferase is a multifunctional enzyme involved in membrane lipid turnover and degradation while synthesizing triacylglycerol in the unicellular green microalga Chlamydomonas reinhardtii. Plant Cell 24, 3708-3724.

Yu, S.Y., Li, H., Tong, M., Ouyang, L.L., and Zhou, Z.G. (2012). Identification of a $\delta 6$ fatty acid elongase gene for arachidonic acid biosynthesis localized to the endoplasmic reticulum in 
the green microalga Myrmecia incisa Reisigl. Gene 493, 219-227.

Zienkiewicz, A., Rejón, J.D., Alché, J.D.D., Rodríguez-García, M.I., and Castro, A.J. (2014). A protocol for protein extraction from lipid-rich plant tissues suitable for electrophoresis. In Plant Proteomics: Methods and Protocols, pp. 85-91.

Zienkiewicz, K., Du, Z.-Y., Ma, W., Vollheyde, K., and Benning, C. (2016). Stress-induced neutral lipid biosynthesis in microalgae - Molecular, cellular and physiological insights.

Biochim. Biophys. Acta - Mol. Cell Biol. Lipids.

Zinser, E., Paltauf, F., and Daum, G. (1993). Sterol composition of yeast organelle membranes and subcellular distribution of enzymes involved in sterol metabolism. J. Bacteriol. 175, 28532858.

Zorin, B., Grundman, O., Khozin-Goldberg, I., Leu, S., Shapira, M., Kaye, Y., Tourasse, N., Vallon, O., and Boussiba, S. (2014). Development of a nuclear transformation system for oleaginous green alga Lobosphaera (Parietochloris) incisa and genetic complementation of a mutant strain, deficient in arachidonic acid biosynthesis. PLoS One 9, e105223.

Zybailov, B., Mosley, A.L., Sardiu, M.E., Coleman, M.K., Florens, L., and Washburn, M.P. (2006). Statistical analysis of membrane proteome expression changes in Saccharomyces cerevisiae. J. Proteome Res. 5, 2339-2347. 


\section{List of Abbreviations}

\begin{tabular}{|c|c|}
\hline${ }^{\circ} \mathrm{C}$ & Degrees Celsius \\
\hline$\%$ & Percent \\
\hline $1 / 2 \mathrm{MS}$ media & 1/2 Murashige-Skoog media \\
\hline A & Light absorption \\
\hline APS & Ammonium persulfate \\
\hline ARA & Arachidonic acid \\
\hline cDNA & Complementary desoxyribonucleic acid \\
\hline CTAB & Cetyltrimethylammoniumbromide \\
\hline C-terminus & Carboxyl-terminus of a peptide \\
\hline d & Day \\
\hline DMSO & Dimethyl sulfoxide \\
\hline DNA & Desoxyribonucleic acid \\
\hline ER & Endoplasmic reticulum \\
\hline FID & Flame ionization detector \\
\hline$g$ & Earth's gravitational force \\
\hline GC & Gas chromatography \\
\hline $\mathrm{h}$ & Hour \\
\hline HEPES & 4-(2-hydroxyethyl)-1-piperazineethanesulfonic acid \\
\hline $\mathrm{L}$ & Length of a protein's amino acid sequence \\
\hline $\mathrm{LB}$ & Lipid body \\
\hline LB media & Lysogeny broth \\
\hline LDSP & LIPID DROPLET SURFACE PROTEIN \\
\hline MCS & Multiple Cloning Site \\
\hline MES & 2-(N-morpholino)ethanesulfonic acid \\
\hline MGDG & Monogalactosyldiacylglycerol \\
\hline $\min$ & Minute \\
\hline MLDP & MAJOR LIPID DROPLET PROTEIN \\
\hline mRNA & Messenger ribonucleic acid \\
\hline MS/MS & Tandem mass spectrometry \\
\hline MTBE & Methyl tert-butyl ether \\
\hline napA & Napin A \\
\hline $\mathrm{N}$ & Number of proteins detected in a sample \\
\hline $\mathrm{NCBI}$ & National Center for Biotechnology Information \\
\hline NSAF & Normalized spectral abundance factor \\
\hline N-terminus & Amino-terminus of a peptide \\
\hline $\mathrm{OD}_{600}$ & Optical density at $600 \mathrm{~nm}$ \\
\hline OGP & OIL GLOBULE PROTEIN \\
\hline PAF & Protein Abundance Factor \\
\hline PAGE & Polyacrylamide gel electrophoresis \\
\hline PCR & Polymerase chain reaction \\
\hline
\end{tabular}


PDAT

Pfam

$\mathrm{pH}$

PT media

PTFE

PTM

PUFA

qRT-PCR

RNA

SDP1

SDS

SpC

TAG

TE buffer

TEMED

TFAs

TGL

WT
Phospholipid:diacylglycerol acyltransferase

Protein families

Power of hydrogen

Pollen tube growth media

Polytetrafluoroethylene

Posttranslational modifications

Polyunsaturated fatty acid

quantitative real time polymerase chain reaction

Ribonucleic acid

SUGAR DEPENDENT 1

Sodium dodecyl sulfate

Spectral count

Triacylglycerol

Tris EDTA buffer

Tetramethylethylenediamine

Total fatty acids

TRIACYLGLYCEROL LIPASE

Wildtype 


\section{Appendix}

9.1 Supplemental figures

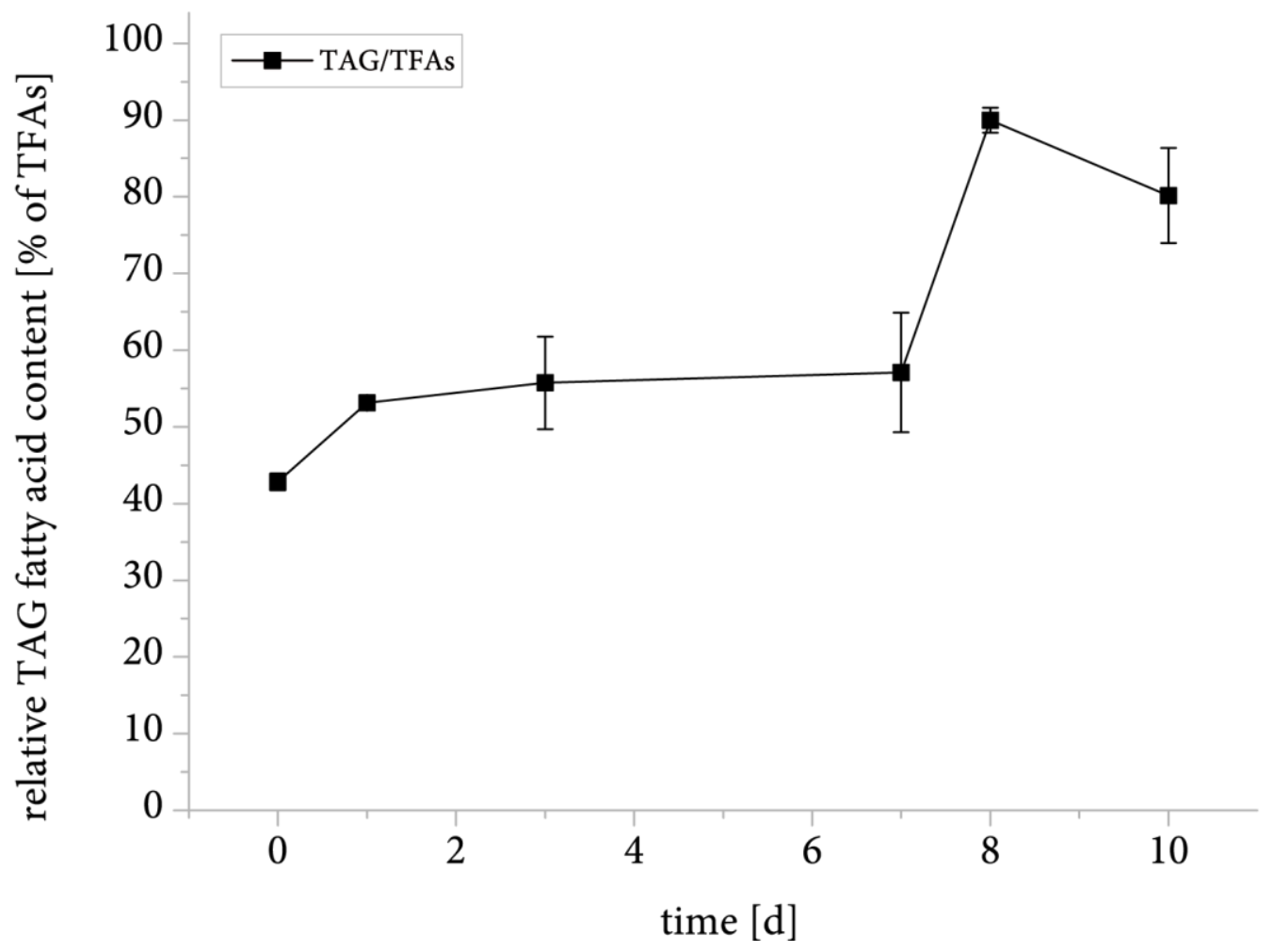

Supplemental figure 1: Proportion of fatty acids accumulating in TAG during cultivation of L. incisa in nitrogen deplete and replete conditions. For each of three L. incisa batches cultivated in parallel in a single experiment, the amount of fatty acids in TAG is shown relative to the TFA content and error bars represent the standard error of the mean. 
Appendix

A

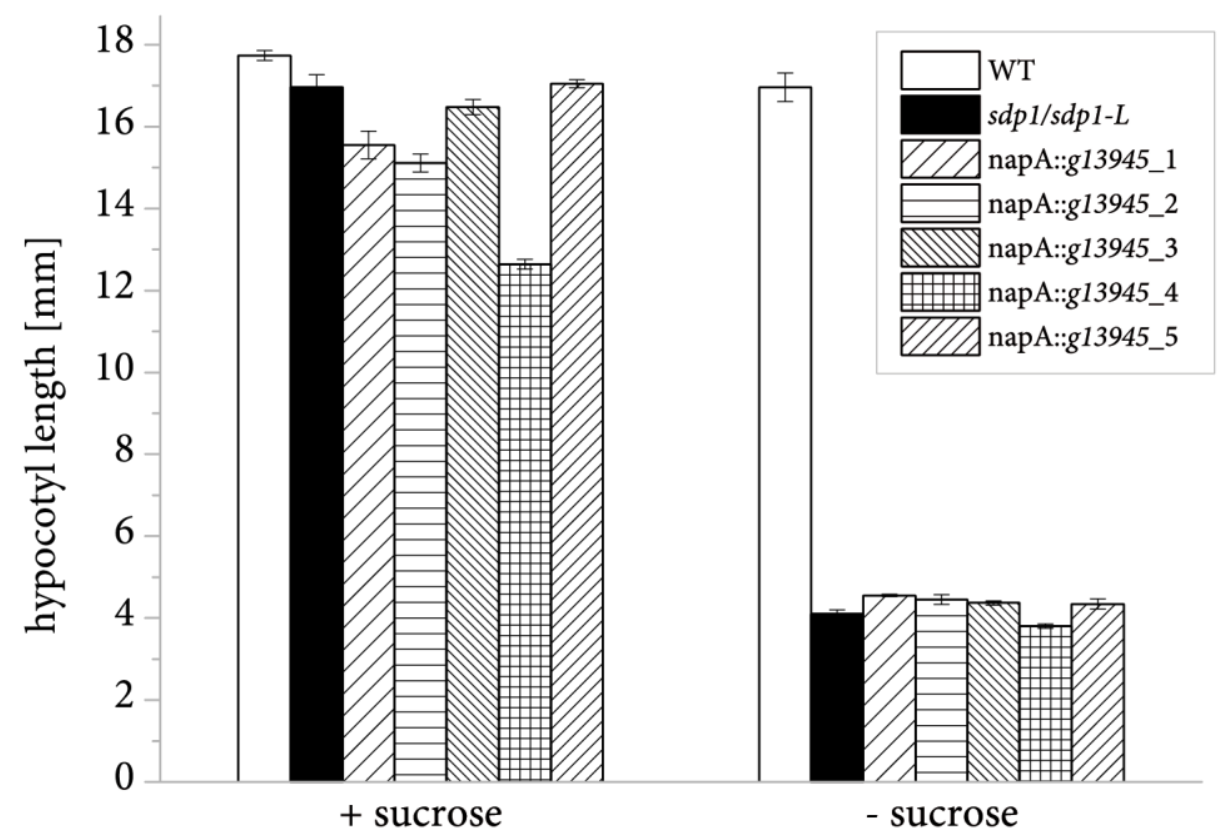

$\mathrm{B}$

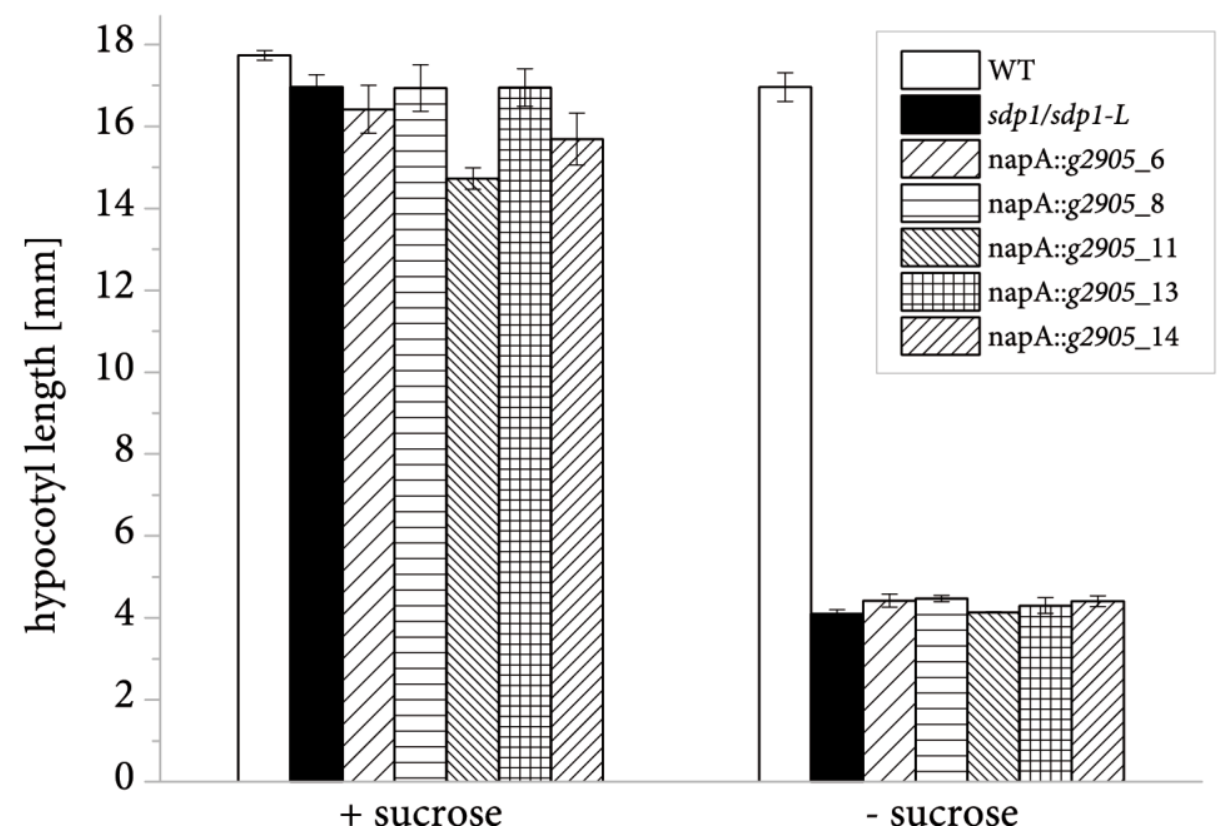

Supplemental figure 2: Hypocotyl length of $A$. thaliana seedlings after $5 \mathrm{~d}$ of germination in the dark. Seeds were germinated on $1 / 2$ MS agar plates either with or without $1 \%$ sucrose. Error bars represent the standard error of the mean of three or four batches of at least 15 seedlings each in a single experiment. A, sdp1/sdp1-L seedlings with the Brassica napus napinA (napA) promoter controlling expression of the $L$. incis a g13945 gene in five independent lines are compared to the mutant carrying an empty vector as well as the wildtype (WT). B, sdp1/sdp1-L seedlings with the napA promoter controlling expression of the L. in cis a g2905 gene in five independent lines are compared to the mutant carrying an empty vector as well as the wildtype (WT). 


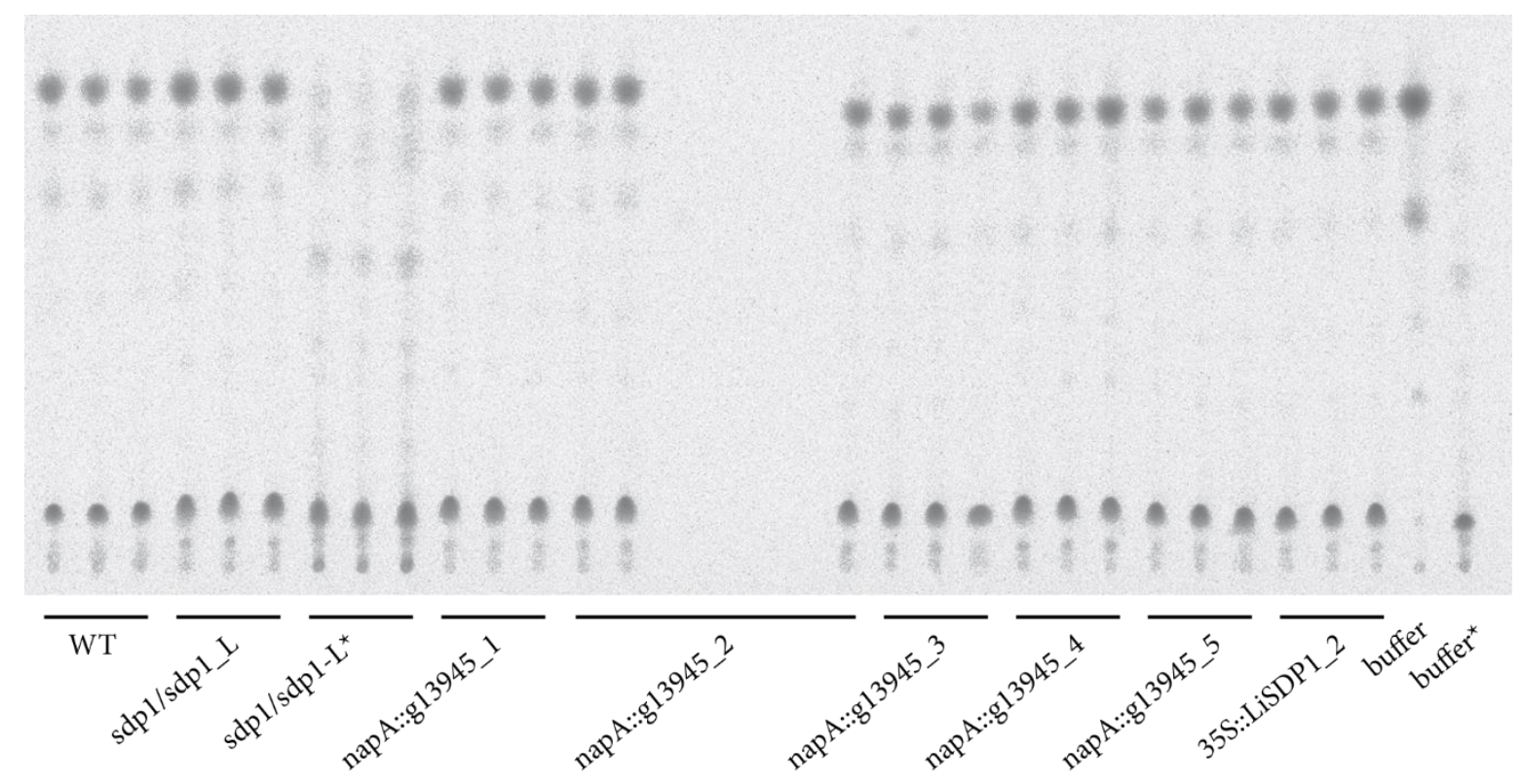

Supplemental figure 3: Radioactivity detected on thin layer chromatography plates showing degradation of ${ }^{14} \mathrm{C}$ labeled triolein in crude seedling extracts at $\mathrm{pH}$ 7. The asterisks denote samples that were supplemented with ${ }^{14} \mathrm{C}$ labeled palmitic acid (16:0) instead of ${ }^{14} \mathrm{C}-\mathrm{TAG}$. The result of a single experiment is shown. 
LiSDP 1

AtSDP1

AtSDP1-L

LISDP 1

AtSDP1

At SDP1-L

LiSDP 1

AtSDP 1

AtSDP1-I

L I SDP 1

At SDP 1

AtSDP1-I

LiSDP1

AtSDP 1

AtSDP1-I

LISDP 1

At SDP 1

AtSDP1-I

LiSDP 1

AtSDP 1

At SDP 1 - I

LiSDP 1

At SDP 1

AtSDP1-I

LiSDP 1

AtSDP 1

AtSDP1-I

LISDP 1

AtSDP 1

At SDP 1-I

LiSDP1

AtSDP1

AtSDP1-I

LiSDP 1

At SDP 1

AtSDP1-I

LiSDP 1

At SDP 1

At SDP1-I

LISDP 1

AtSDP 1

At SDP1-I
MKPLTYQTGRPPVCRTS S GLPGATALLD LTLVRLVATLSVPFRK LUAFNLFIGRAPLQIL

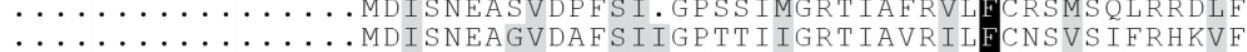

\section{QLRKRLRQATATATNYQDWSIAATALDQLQGNDS LSRWKRETRLYDRKLLQERLS HLQEV 97 YRRKEWRNMMRAALTYEWSHAAKMLDKETPK . . . . . V VNETDLFDVELVSNKLDELKHR}

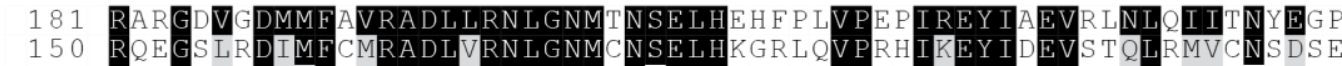 151 RHEGSLRDI IFCMRADLVRNLGNMCNPELHKGRLHVPRLIKEY IDEVS T QLRMVCDMDTE}

\section{DISAAEKLeFLeETRHAFGRTALVLSGGGALGAFHLGVVKTLEEQKLLPRVLAGSSVGS I \\ 210 ELSLEEKLSFMHETRHAFGRTALLLS GGAS LGAFHVGVVRT LVEHKLLPRI IAGS SVGS I} 211 ELSLEEKLSEMHETRHAYGRTALLLSGGAS LGAFHLGVVKTLVEHKLLPRI I A GS SVGSV

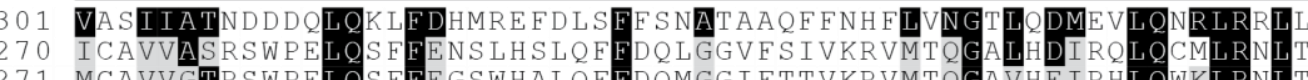
271 MCAVVGTRSWPELQS EE GSWHALQFEDQMGG IFTTVKRVMTQGAVHE IRHLQWKLRNLT

647 SASSRARNSLRGR GMGGRI PSWLHLPSLGMPTVPS DESLDSYSLAVRAGST D DLPGLRA 624 LVTDN........NLHASSGRNLS............ DSETESVELSSWT.RTGGPLMRTASAN 628 DVLAEASRLYQH IVVGSGRNSNRT SNLSHTYDAGSECDSPEAEDWT.RSGGPLMRTNSAQ

707 TDSPTKASSGSKSDLISELISSYSSRPLPEVPEVEASLEGLPAPEFPEWSGSAAFDCCD 666 KFIDFVQSLDIDIALVRG ESSSPNSPAVPPGGSFTPSPRS IAAHSDIESNSNSNNLGTST

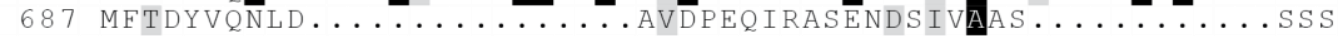

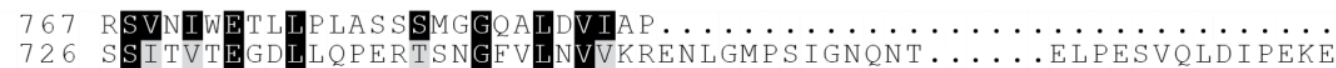

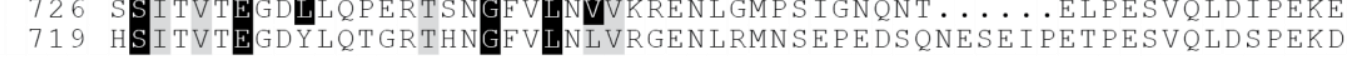


Supplemental figure 4: Features of the L. incis a SUGAR DEPENDENT 1 (LiSDP1) amino acid sequence. Similarities with the amino acid sequences of $A$. thaliana SDP1 (AtSDP1) and SDP1-LIKE (AtSDP1-L) are shown with black and grey boxes highlighting identical and similar residues, respectively. Sequences were aligned using MultAlin and ExPASy BoxShade software. Components of the active site are marked in red. The grey bar represents the conserved patatin domain. 
A

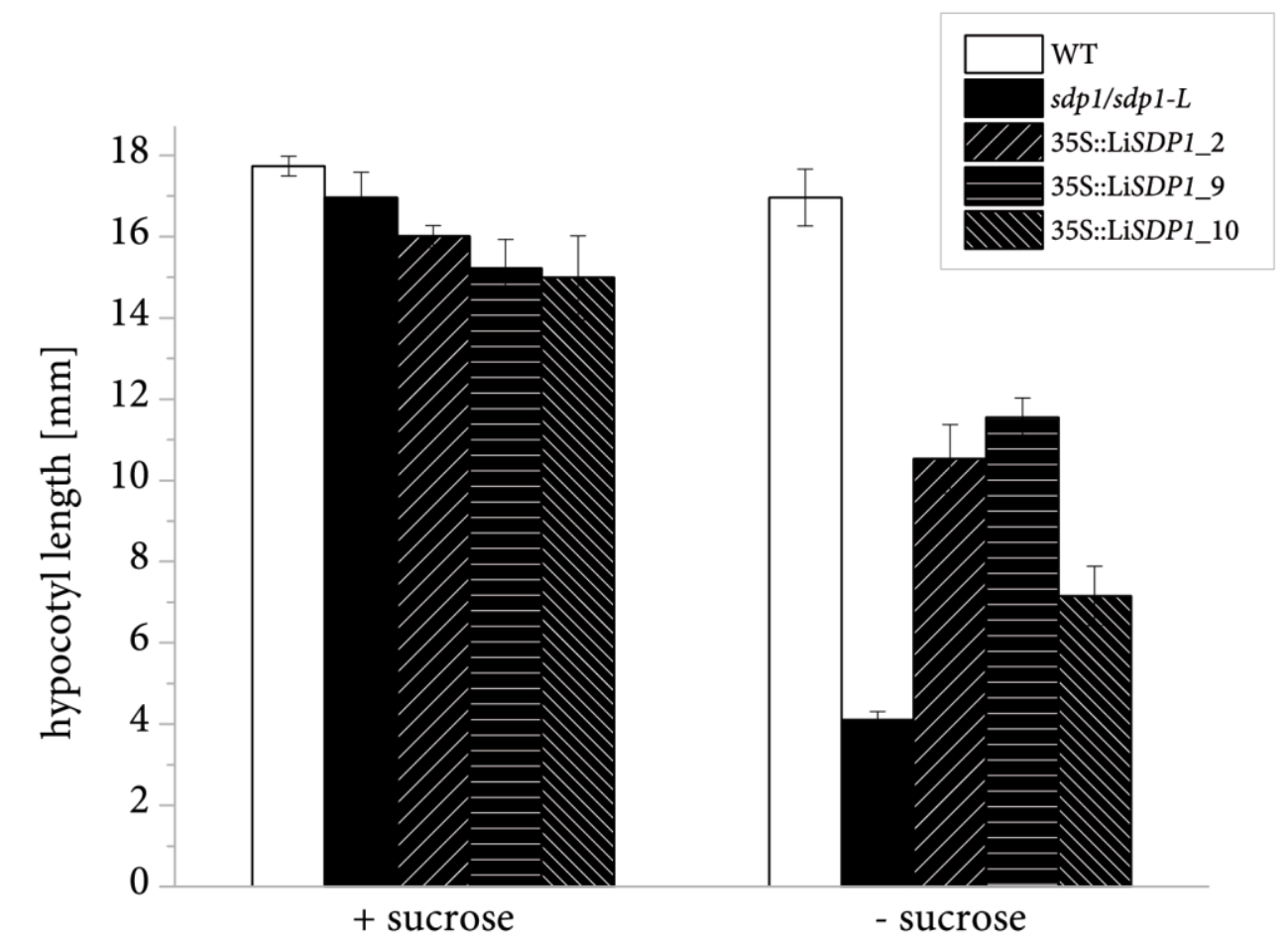

B

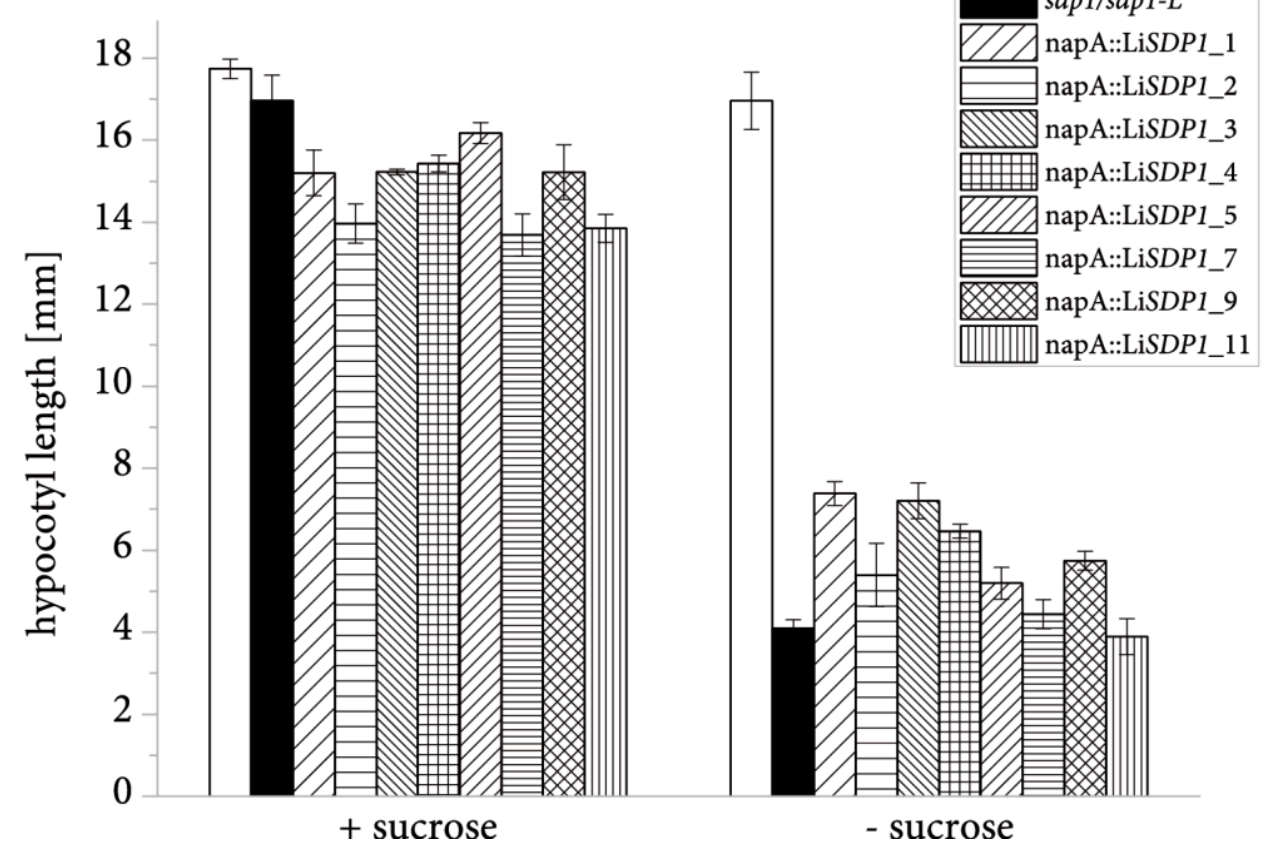


Supplemental figure 5: Hypocotyl length of A. thalia na seedlings after 5 d of germination in the dark. Seeds were germinated on $1 / 2$ MS agar plates either with or without $1 \%$ sucrose. Error bars represent the standard error of the mean of three or four batches of at least 15 seedlings each in a single experiment. A, sdp1/sdp1-L seedlings with the Cauliflower Mosaic Virus 35S (35S) promoter controlling expression of the L. incisa SUGAR DEPENDENT 1 (LiSDP1) gene in three independent lines are compared to the mutant carrying an empty vector as well as the wildtype (WT). B, sdp1/sdp1-L seedlings with the Brassica napus napinA (napA) promoter controlling expression of the LiSDP1 gene in 8 independent lines are compared to the mutant carrying an empty vector as well as the WT. 
A

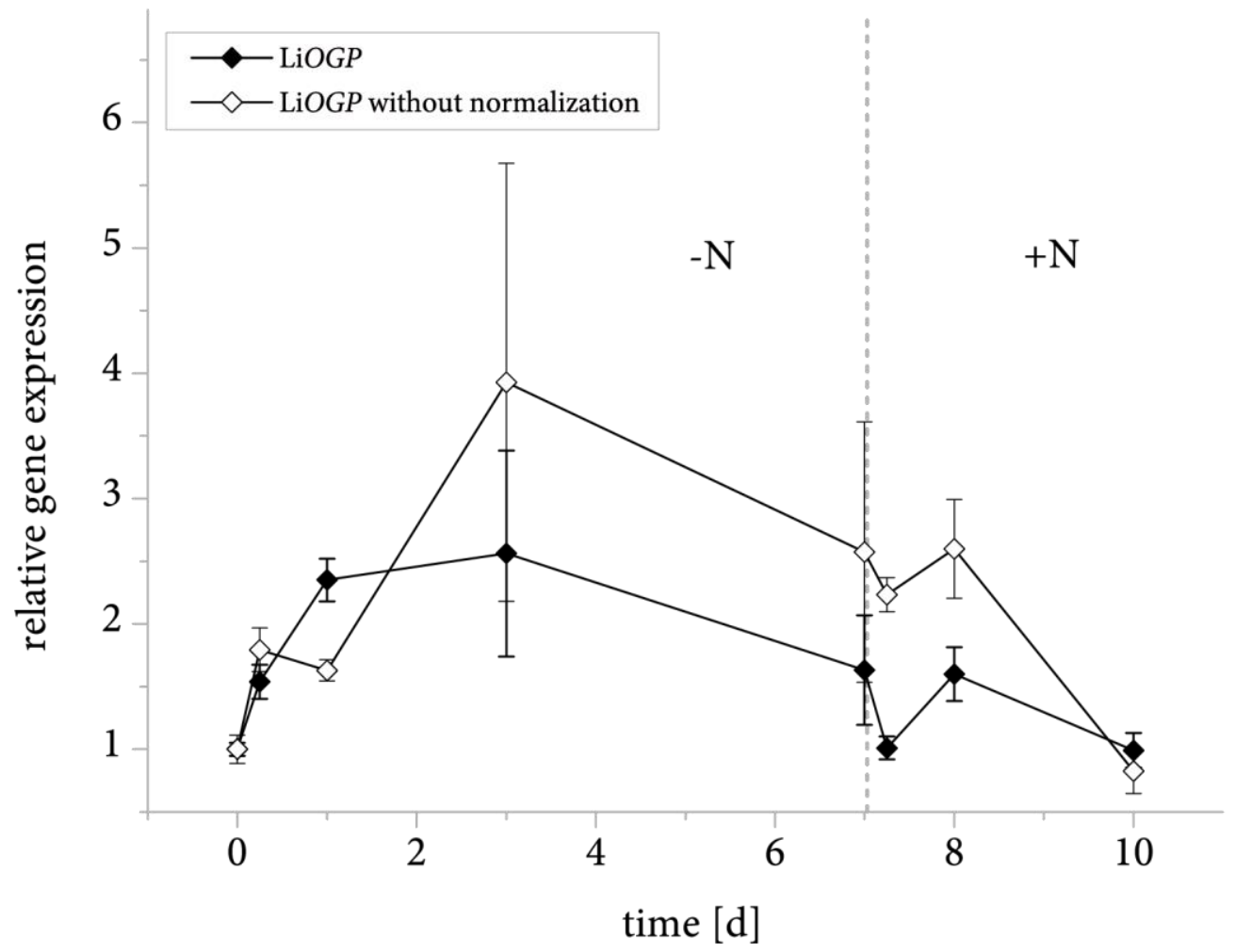

B

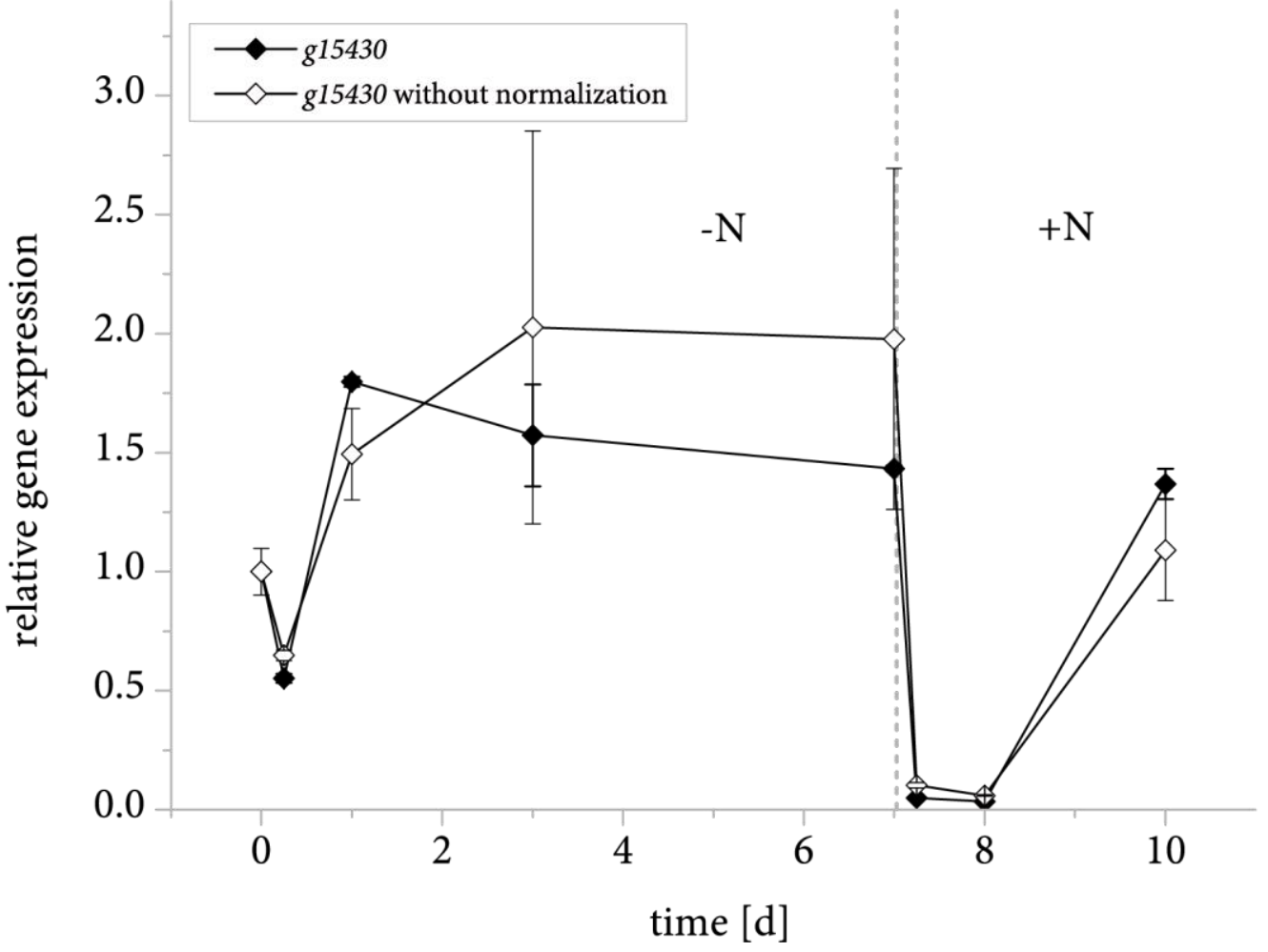

Supplemental figure 6: Comparison of gene expression as determined by qRT-PCR with and without normalization to RIBOSOMAL PROTEIN S21 transcript levels. Expression is shown relative to time point $O$ and error bars represent the standard error of the mean for three batches cultivated in parallel in a single experiment. The dotted line indicates nitrogen resupply. A, L. incisa OIL GLOBULE PROTEIN (LiOGP). B, g15430. 
Appendix

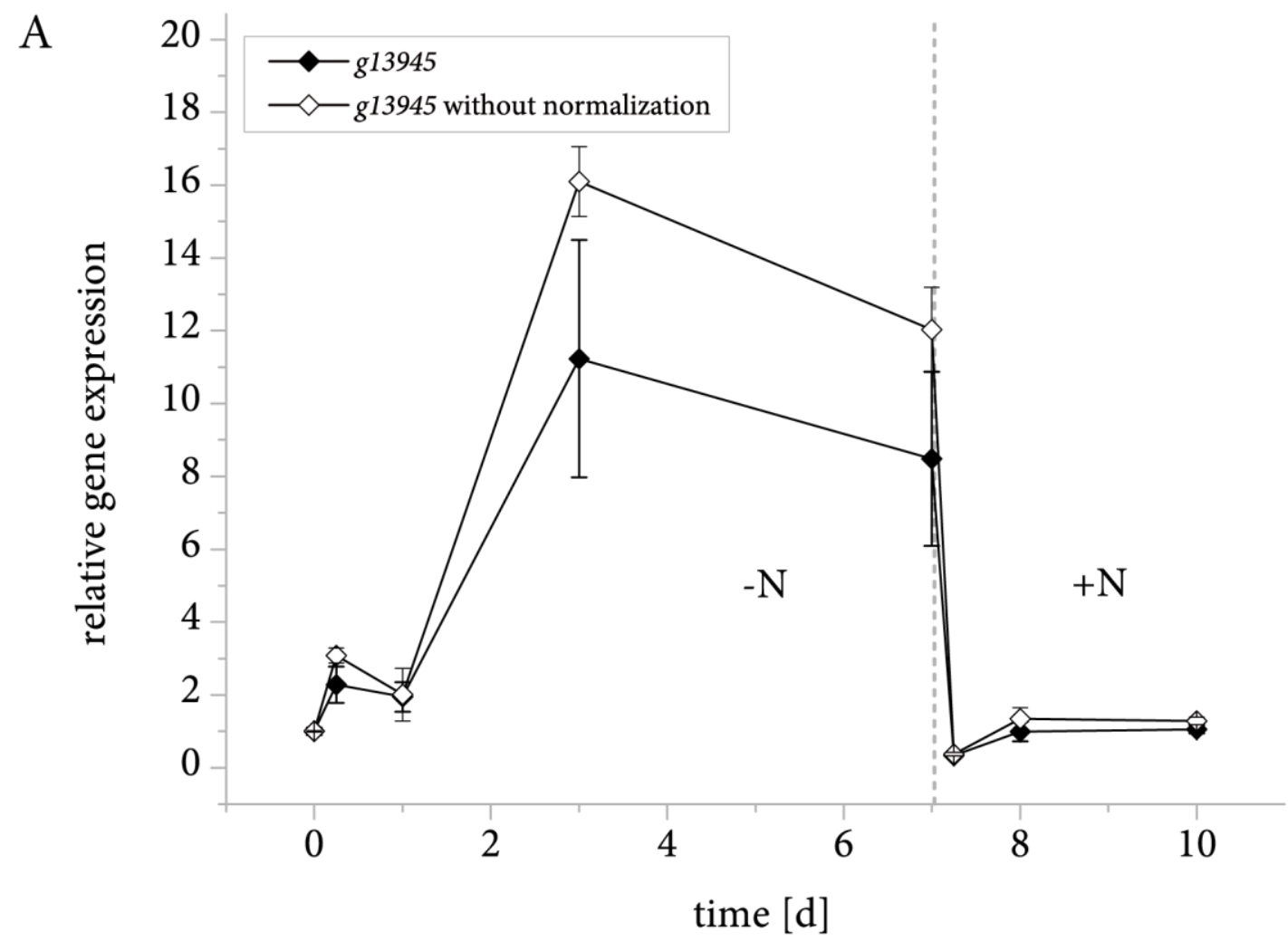

B

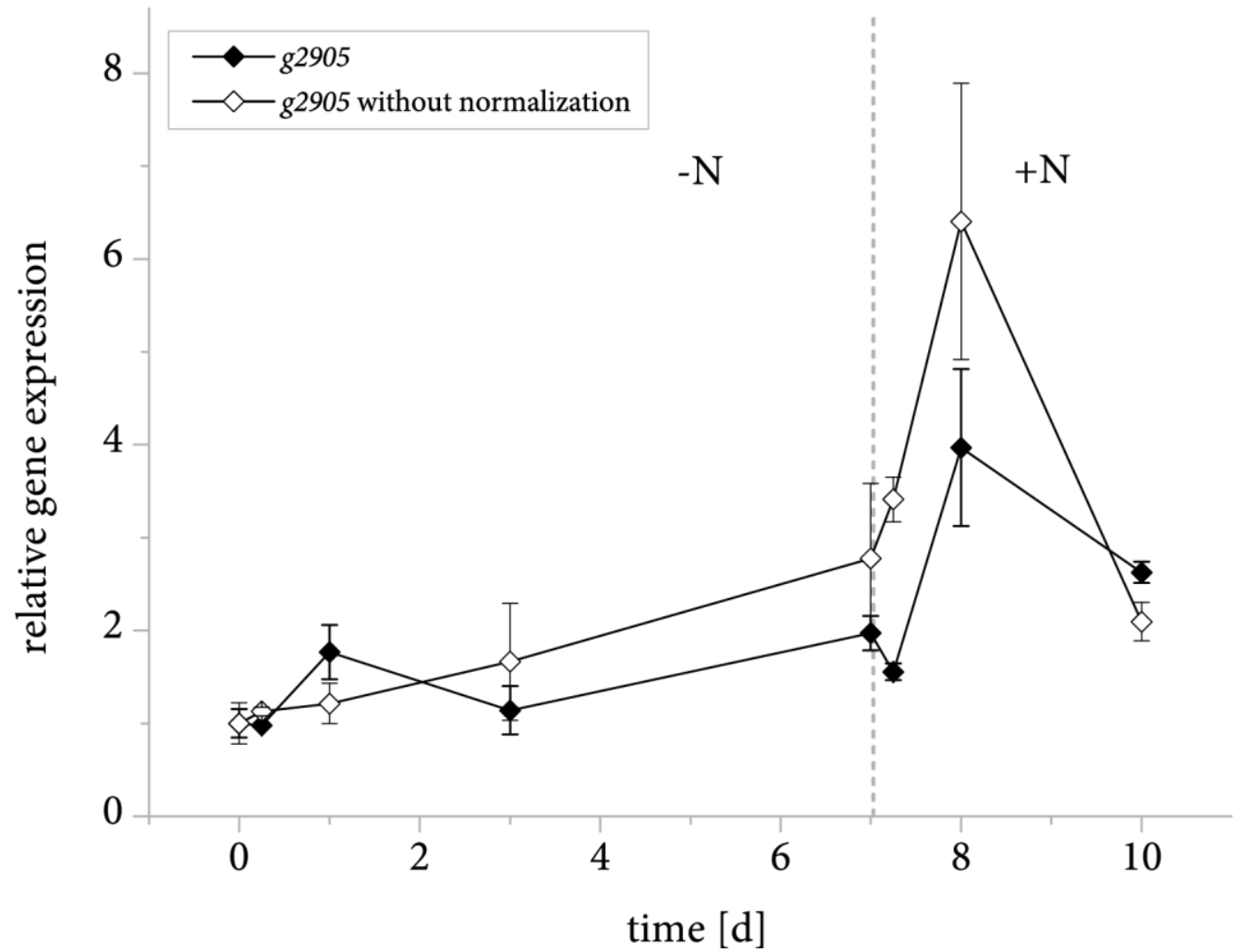

Supplemental figure 7: Comparison of gene expression as determined by qRT-PCR with and without normalization to RIBOSOMAL PROTEIN S21 transcript levels. Expression is shown relative to time point $O$ and error bars represent the standard error of the mean for three batches cultivated in parallel in a single experiment. The dotted line indicates nitrogen resupply. A, g13945. B, g2905. 
Appendix

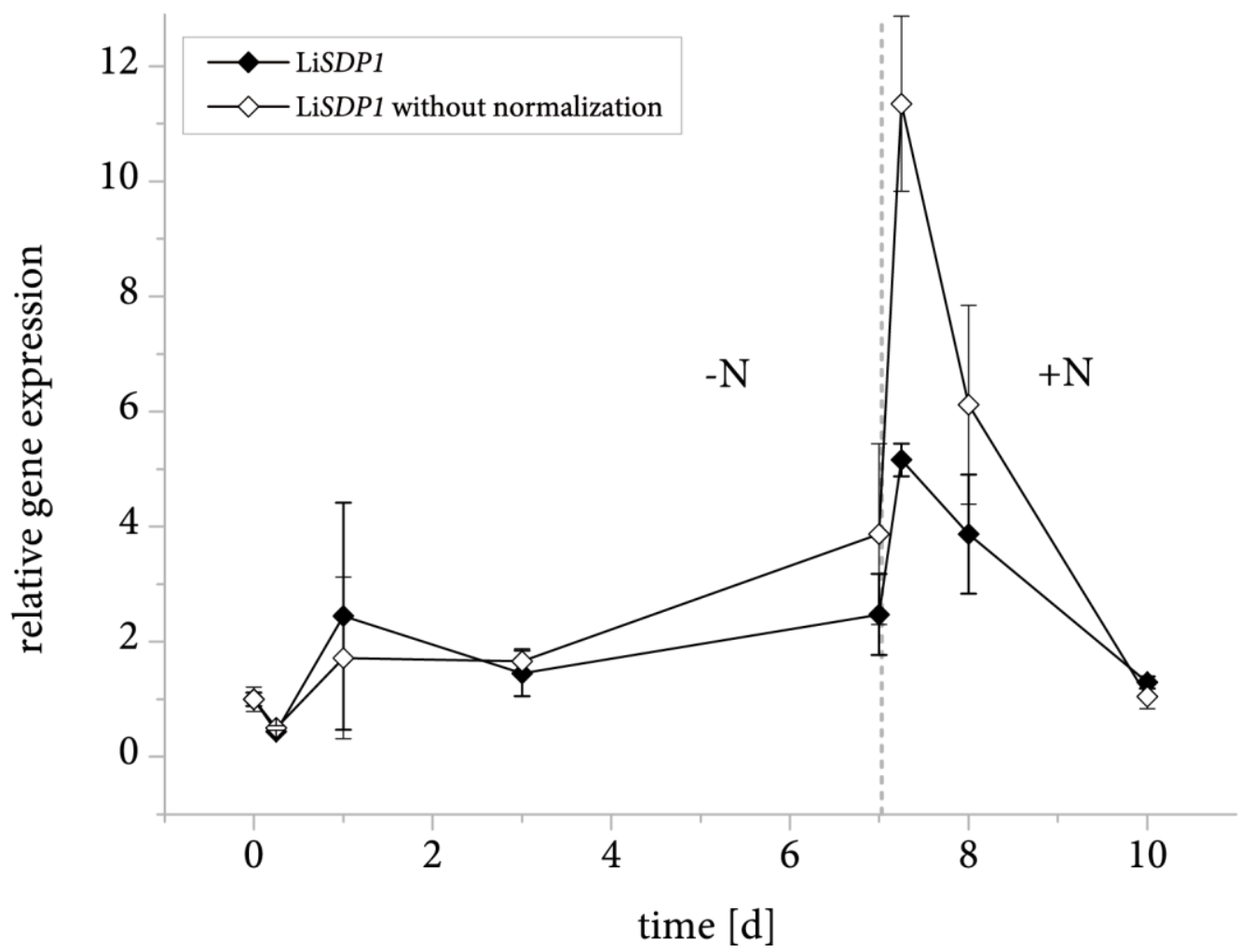

Supplemental figure 8: Comparison of LiSDP1 gene expression as determined by qRT-PCR with and without normalization to RIBOSOMAL PROTEIN S21 transcript levels. Expression is shown relative to time point $O$ and error bars represent the standard error of the mean for three batches cultivated in parallel in a single experiment. The dotted line indicates nitrogen resupply. 


\subsection{L. incisa coding sequences used in this study}

g555.t1

atgggtgctgagccagtgaacaacgectctggcgctgacctgaagcgectggctttcgtccgctcttacagcgactactacttccagaaggg ctatggcctcgcctcatctgtctacagtactggcaagagctatgcaccagatcgcttccacggcagcatcgecgctgtagagcagacagtca gcgagtatggaacacccetggtcaccgcggtccaggacaaatctgaccaagtgctgcaccttgtggactccaaggttgacaccgctgtcact tccgcctaccagttctacgaggacaagtccagcttcctggecagccaggtcgagaagcagaagaagtatcatgaaaagaacctggagcact acaaggccagccgcgaaaactacctgaagaagatcgaggagagcgtggacttcctcaagaaggagggcctcaccggcagcgcgcggtac gccgccgacgccgtgctggctcgcgtggatgatgccaagaagttgccgcccatgctggtggacgaggccaaggtggtggtcagcaaggtc ggcgacgcctgggectacctggcttcactgccagcagtccacaagctgctggagactgcacagccctcactggatctggcctggaagaagt acctgatcgeccatgacagcctgctgtccacccccatctaccaccagatcgeccgggactccaccgctgtggtgggeaaggtccagcaga cccccatctacaagaagatcacaacgacagtctaccccatcatctctccetacgetgaccccgccctggacacgatctaccagtcaagctac tactctgctgtcaaggaccacctaaagcctgtggaggtgaacggcgctcactga

g2905.t1

atgtactcagcgatgcagttggcagtgcttgctgcaagcctgctaactgcagctgctttgaacagccagcaagtaggtatgcaactggctggt acacaggcctacgcgatgcctgaggtgctgggcgctgatggttccettcagcgcactcacagtatggcagagctggtgctgccagagggct accttctggaagagcatgtcgttgagactgcaga gggctacctcttgtccctgtaccgcatacccaagggccgctttgacgcaccgtccagt cagccaactcacaggccggtcatctacctgcagcatgcactgctggactgcagtgccagctgggtcaacaacggcgcagatgccagcctg gccttcatcctggccgacgcaggctatgacgtctggatgggcaacagccotggcagcacgtacgccaggcgccacatcaatctgtcggtg gactcgcctgagttctgggcattctcctgggacgagatggcagccttcgacctgccogccagcatagactttgcgctggcgacagcaaacc agacaaagctggcgtacataggccactcacaaggcacaactattggcttggctgccctcagctctcagccagaattgcaagagaaggtgtc cctggcagtgatgctggcaccggtcaccttcctgggccacatcgctagcaggccagtgcaagctcttgcgcgcatgcagactgatcagatat tccagctgctgggtgtcaaggagtttctgccttcgcaagaggcgagcattgagatctatggggagatctgccocacagcgcccagcacatg cattagcgccattggectgatttgcggctacaacccagacaacatcgaccagtcgcggetgccactgtacctgtcctacactcctgcaggta ccagcgtacagaacatggtgcattggagccaaggggtgcgtcggccatatcccgcctttgcceggtttgactacggccacgactgcacaag tccgcttgggctgccccaaaagtgcaaccagaatatgtatggcaccaatgaagtaccactgtacgacctgaagcagatacgcataccgettg cgctattctcaggtggacaagacatgctctctgatgctcaggacatcggtgtactgacttccgcgctgtccgcggagcacatcgtgtttgcgc atgtgatcgatatctatgagcatttggactttacgtggggcatcaatgctcatgaactcgtgtacagggcgatcaagcagctgctgtggcagta tgccaggttcccgcagggctaa

g5830.t1

atgctgcgccaggcegcctccagcagcttggcagctctcagagcaacgagcttgcaggcctgcggaaactcggcagcaagggacgctgg cagtaatgttatggtcagctccaccatggcgcagcattgctggcaccagcagcgaggcctgaaggtgccagtgcagaacaacaacgtagac aaggccttttcccagctgagcaggaagctgagggccgaaggcatgatcgacaagtggagggaccaggtggagttcaccaagccagccttg aggcgagtgctcaaccagaaggagacagcgcacaggctgaaggtggagcggttcagggcactgatgagctgggttctcaagcgcaaagc caggggcttctga 
g9192.t1 (LiSDP1)

atgaaacctctgacttaccaaacggggtcgcccaccagtctgccgaaccagttcgggacttcctggcgcaacagcactgctggacttgacctt agtgcgectggtcgecaccttgtcagtcccettccgcaagctacttgcattcaacctcttcatcggcagagcacccetgcaaatcttgcgttat gctctctactggataggatggctatctgctcogcctcgcacgccgctgctgcgtcacctgggcacagactttctgcagacaaggcggtggca ctttggcctaggcacggtcctcctggctgccgctgtctctgctgctcggcggaaatggctggaaactaccagcacagatgctcagctgcgca aaaggctgcggcaagcaacagctacagccacgaactaccaggactggtcaatagcagcaacagctttagaccagctacagggcaacgact ctctgagccgatggaagcgggagaccaggctgtacgatcgcaagctgctgcaggagcggctgtcccacttgcaggaagtgcgagctcgcg gcgatgtggggggacatgatgtttgctgtgcgggcagacctgctgcgcaacctgggcaacatgaccaacagtgagttgcacgagcacttccc gctggtgccogagccgatccgcgagtacattgcagaggtccgcctgaacctgcagatcatcaccaactatgagggcccagacataagtgc ggcggagaagctgcagttcctgcaagaaacgcggcacgcttttgggegcacagcgctggtgctgagtggtggtgecgcgctgggcecttt ccacctgggtgttgtcaagacgctctttgagcagaagctgttgccgcgcgtgttggcagggagcagtgtggggtcaatagtcgcatccatca tcgcaaccaacgacgacgaccagctgcagaagctgtttgaccacatgcgcgagtttgacctgtccttcttcagcaacgcgacggecgegca gttcttcaaccacttcctggtcaacggcacgctgcaggaca tggaggtgctccagaaccogctgcgccgcctgctgggegatgagacgctg ctgcaggectacacacgcacaggecogggtgctgaacgtcgcggtcacggetgctgacaccaacgagcctccgcgcatcctcaactacctg acggegcccaatgtggtcatctggagcgeggtcgectgctcctcagccttccegctgctgtacacgeccogcaagctcttcgcaaaggaca ccaagaagaacgtgctcgtgccgttcacggcggaggcgatgcgcacgagcgagcggcggtggcgggatggcagcctggaggaggacct gccgatgcgcacgctgagcgagatgttcaacgtcaactacttcctggtcagccagaccaacccgcacatcgtgccggtgctcaacctcaaa aagcgcttcaaccgcaagctgggcaacctgctggaggctgaatggaagcacaggtgtaagcagctggtggagatcctgccgactgggatg ggcaagttcctcaaggtgttcagccaggtgtgggagggcgacgtcaccatggtgctgcctccaccttctggcagctacgcaaggccatca ccaacccctccaaggacgacctggtagcagcctgccgccagggtgagcaggtgacatggtccaagctgagcgccatccaggccaactgt ggcatagaaacgacgctggatgagtgcctcatcaagctgtcagagaccatgtttgcgcagaagcgccagcggagcgtgggcagcacggcc agctcaccgatgaaggtcatgggcggggaccagttcccaggcccagggatggcgctggggaccagcgcgccoggcaccagtgcgtttgt ggcggccggcggcagcgaggagccgttggcgtacagtgcaagcagtcgggctcggaacagcttgcggggtcgcaacggcatgggaggc cgcataccctcgtggetccacetgccctcgctaggcatgcccacggtgccttcagacgagtcgctggacagctacagcctggcggtgcgtg ccggetcgactgatgacctgcceggcttgcgtgccacggacagccccaccaaggccagcagcggcagcaagagtgacctcatctcagag ctgatctccagctactccagccgacctttgccagaggtgccggaggtggaagccagcctggagggtctgccggcgeccgaggagttcccc gagtggtcgggctccgcggcctttgactgctgcgatcgcagcgtcaacatctgggagacgctgctgccoctggcatcotcgtccatggggg gccaggegctggacgtcatcgegccatga 
g13747.t1

atgactaagtccacctcatcccgatcagcacgtgatgtggecttcagtgtcttcgaggtcacgacagcagtgatgtatggegtgtacgagaac actctggccggcgttgtgccctacttcgtcccggatcggacccgggaagccttgttccagctgttctgcgagctcaggaccaactctcccaa ggacaagcggtttttgaaaaagcacctggcggcgttcgttcccgagaccgtcactggctggatcgccatcgcctccagtgctttgtatgctac cagcgtagtcgccggcagcctgttcatgctggcgggcctggcatcagccgcgctgttcagttgcgtcggcatcgtactagttacaggcagct ttgctggcgtagcgctggcgttcctggcactgggtctcctctgtgctgcgtgcatcacaggcgtcatgggaactgcgacagcgtttggctacc tgtctgcgctcaccggcagcgctgcctggcagttcgtcagccagcatgtgtttggcggggcttcgctggtccoggactccattcgtgacctg cccatttccaggcacgctggcaagatcttcggctctactgcttctgcgcacaagcagagtgacaccaagcgcgcctcagctcgcgcctcag ctcaacagccaccctctgcccegcagccaccgaagcttcagcccctgagccagcaacaggcattcggctcaatgcagacccaggatgagg ctcagtgcaccggcaagcagccaacagctggtcaggcacccagcgagccgcagcaggcaaagcatgcaaagcagccggcaccagcog agctgccacagccagtcagtagcgtggectcagtgaaggeccctgatggccccacaacccctgcgccaagcgacaccaagccegctgtg gegctgccgaccacagtgcctcagctcgcattgcccacacctggctcgcgcagcacagcggtgcagccgccttcaagcttcaggatttcaa ttgactcagcgccaagcggcgactcogccgagacgggtgcatcatccagggcagcagacgcaaacgatgcaggccaggatgcacagcc cgaaactgacgcccagcacgcgcagcatgtcaagcaacctgcaccagccgagctcgccaagcctcccacaaccgagcccacagctgagg ttgtatctgagcagcccaaagcagcgcccaaggtcgettttgctgacccacagcaggccatcactgctagcagtgcttctgctgaacagctg taccaaatcaagccaagcgcggtggaccagcagcccgtgacagcccggcatggcacagcggecactgaggcgcagcaggccaagcatg cacagcagccggcaccggcagagcctggcaagcctcccegcagcgagacgcctgctgcggecgetgcgccegcagcgcagcagacac agcgcagcgtgccagaacccatcgacgtgcacatcattggcgatgcggatgctgcacaggctggeccgccacctggcgcagcgectgcc acgcgctctaacgccagcaagtcacaggagctgtttgcggacctggccgacacagctgtggtggaagccatcgatgatgegecgcacgag atcgatgccagtgtggecctcaacagtgccctcaaaacaggcactgcacaggcggctgttgtggagggaccagcgectgccatccaggcc agcgcctcagcaacacacagcgatgatacgggettcctgacaggtgctaagggtgtcgcaccgaactacctgagcacgcccgacagcaac agcaagctgccagctggtctgcagacgtccetgccagccagcatcgacacgcagccggcgccgttctcctttgtggtgcctgagectgcga ctgctgctccacacaccagcagcgetgctgtgctgacgggcctacggtctgctgacgtggcacctcagggtccgcacctggctgcccatgc ggaccagcctgcggcacccatctttggccacagcgcagcgcagcagcctcagcaggcagacacccaggcccetgcctcggccatggggt tcagcgeccccagcgtgcatgacaacatccttttcgacctcccccgagcaggttcaaacacccctatgctcagcgacaccaagccgeccgt cgcgctgagcgcgctgccacagcctaccgcggcgatcccegatgcgaccaccacaactgtgcagcccgtcacaagcttcaggatctcaat tgatagcggggactcggcccacacaggcgcgtcagacccgctcgtgcgcagcgaaagtgagggcagtgaggtcagcgcgtccggcagg ccaaagcgcatgtcgtccaagaaccggcggaaggcgcaggcggctgccetggecgctagcgctgaacagtga 
g13945.t1

atggcatctcatgacaacctgcgggecctgcgaggecccegggagggecgcgtggacctgcgecgccccgagaactacgaaagcgtcta tgacaagatggtggacgecttcgtctggctcgctcgcaccttctgcacctgggecgtcccacgcttccagccctgctggacttcatcgtgc accacatctgcgagcctgtcttcgeccgcetgactggcagcttctacccacccaaggeggtcctgcaccgcggccagcagccggtgtcctt catcagcgtcaccgaccccgagtttggcgtgcctctgctggatggcaaaccgctgtacaacccegccaacttcccetttgacgccacgcatg ccgacgctcatgggttccatgagtgggtcgcccacttcctcatcctgtgcgccaaggtggcttacgagcgcgaggcgctgatcaagatggtc gtcgacagggagttcaccggcatcaagtttgtcgacagcttccccacagagcccaaggaggctgccaacttgaccgtggaggagaaggcg catgtgatgcaggaggcggccttcaatcggctcatggccggcgctgacacagcgaacccagcgatgatcaagcggeggctggggtctgag caccagcagctggcgaacagagtcaccgcaaccctcatcceggacaccatggccttcatgatcaccaacgacaatgctgtcatcctgttctt ccgcggcactgagccgcacaagctcgcccagtggtggtccgactgcgatctggagctggcggtgcggaccaacgecgccgggaaggtg caccagggcttctgggaggcgctgttctacaaggcaccgcctgtcaatggcaagaaggagatccccagcgtcttcaaccgcatctgtgcgg cactggagagggagacgaggggcaaccacaagcgcatctacgtcacgggtcactctctgggtggaggcctgactgccatgtttgcccaca cgcttgctcaccctgacactgcaaagctgcccagcaccottgggtacaaggatgctcacaaactcotggaccotgtgggeggggtgtacac tttcgcgagccccacggetggcaaccatgccttctgtgatttccttgtctatacctatggcaagaatgtcaagaagggcattggcaaggacag gcttttccgtgtcgeccactcatcggatgtggtcgtcaagataccttttgggcagggctaccgccaccacaagcttgagtactacatcaactac aaaggcgacatccaccatgatccggaggacatcgaggcatggcgggtgtgcgagagcgaccagttcgacttcttctacctctgcaaggtcc tggccggctggacccgttggtcgccctgggggggcgcccaacggcagctggctgtggccggacttctgggtgttcttcatgegctgctacct gcacaccgtcattcgtgtggtcgcctatacgetggggeccetcatcccaggccatgtcatctccggegtgccegaccacttccegtgcgact acgaacgcaagatccgccgcgtagccgttgacctcggecgcgtgctcaaggectcgeccgagcagcgtacccacatgcacacccatgtcc tctcgcgttccgaggagcactgtgatgetgatgtcaacatccetgtgagcgtcaatggcggccacatccagagcagcaatggectaaacatg aaatga

g15430.t1

atgtataacgcagacgggtccatgcgggccatccgccgtcctgcgcactccagcggaggcggtcagtcaagctggagcgggggttgggc agcagatgcaaaggagaatgagcccegcaacacggtcggctcaaactcaggcaacgttgctggcggtccgtcacctgctgcagctggtat gaagtccaagagcgctggcgctgaggetggcaagggttcacgacagccagctgacgccaagatcaaggcgctgcaggggagcaacatct ttggcaaccacagcgacgcggccactgtccaggacaccactgccaagctccagcaagtgcaggtgcaggacatgcctgccocgggcagc gccacaattgccaactccggcgagtcaggagtccgtgacatcagcgatgccaagcggcgcgcgctctaccagacaaacgtgttcacgcac acggatgagcctgcaaactggegctctgcagaggccattgccagcaagccagaggtccccgcggtcccagctggcagcgctgggcagtt ccgggcagccgagccgggegcgcgcgacatcagcgacgccaagaagaagtcgctctaccagacccgcgtctttggcacggecgatgctg cctcgcccgcagcgcagcaggtgtccagcctgaagcacaaggagatgagcggccacaacatctttggegctgctgaggagcetgegeco cgcgctgctggccagtcgggccggcgccccccaggaggtgccagcaccttcagctttggcaacagcggcgctgagagcgctgcggeccc ctctcaggggggtagccggcagactgctggcggcagcagcacgttcagctttggatggtga 


\subsection{Vector maps}
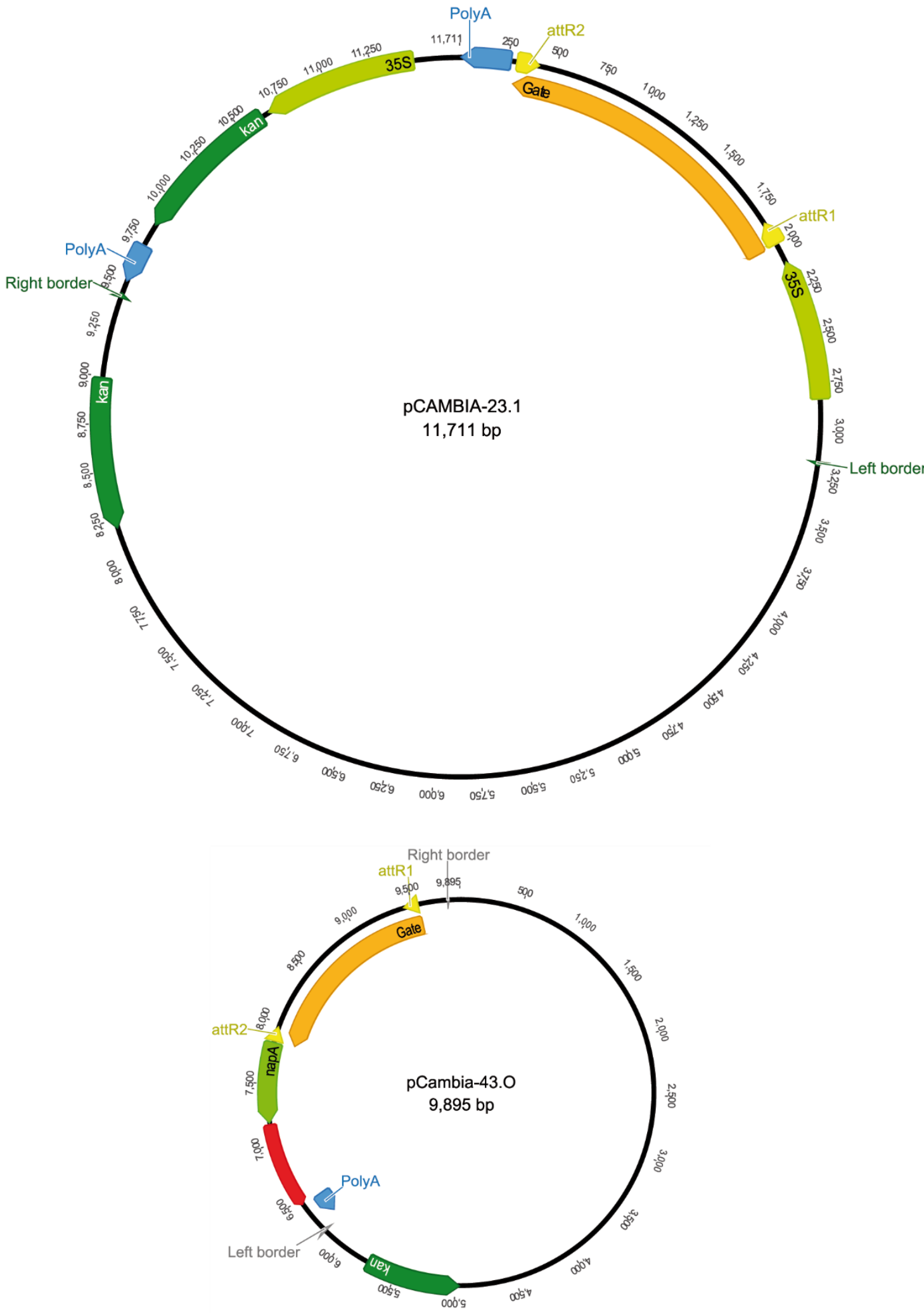


\section{Acknowledgements}

First I would like to thank Prof. Dr. Ivo Feußner for giving me this project and letting it evolve with patience and a great deal of support. Not only were our discussions invaluable for the progress of the project, but your positive way of motivating me certainly made a difference as well.

I am also indebted to Prof. Dr. Lipka and Prof. Dr. Friedl as members of the thesis committee for their interest in the project and very helpful and interesting discussions.

Dr. Oliver Valerius (Institute for Microbiology and Genetics, University of Göttingen) performed LC-MS/MS analyses of the protein samples and I am very grateful for this as well as for his practical and theoretical advice.

I would like to thank Dr. Jennifer Popko for GC measurements and data analysis, her guidance in this work, lots of helpful advice in the lab as well as her patient and kind way of dealing with her students and colleagues. I am also very grateful for her critical reading of this thesis as well as the never ending supply of chocolate coming from her office.

Dr. Ellen Hornung has played key parts in several aspects of my work. Firstly, she is an incredible resource in terms of scientific experience and advice and has helped me out with hints and propositions many times. Secondly, her practical help with cloning, genotyping etc. as well as her critical reading of this thesis have helped me out a lot. Thirdly, she kindly provides half the lab including me with great food. And finally, it is a mystery to me how she stays in such a good mood while working this hard and constantly being asked for help, but I admire her for it and I hope that no student (or ligation reaction) will ever change that.

I am very thankful for all the help and advice I received from Dr. Till Ischebeck. He has introduced me to and helped me with tobacco pollen transformation and has been a great support in confocal microscopy. Despite being extremely busy, he still finds the time to help out, which I fully appreciate.

My thanks also go to Dr. Martin Fulda for his helpful advice and ideas, which have been very valuable in this work.

Dr. Corinna Thurow and Dr. Guido Kriete (Albrecht-von-Haller Institute for Plant Sciences, Department of Plant Molecular Biology and Physiology) were a great support in setting up qRTPCR in our lab and helping me with practical as well as theoretical issues, which I am very grateful for. 
I sincerely thank Sabine Freitag for her practical support with lipid extractions, constant willingness to help out where she can and being a fantastic moral support throughout this work. The lab would not be the same without her and her great sense of humor.

Anna Müller introduced me to radioactive lipase assays and was a great help, thank you! I also appreciate all the practical help that I got from Andrea Nickel and Susanne Mester in terms of cloning, genotyping, plant care, seed collecting - thank you! Thank you also very much to Janett for her help measuring hypocotyls.

Even though the results of their work did not end up in this thesis, I am grateful to Urs Benning, Dagmar Akkermann and Alicia Illen for their work related to L. incisa DGATs during their Master theses and lab rotations.

I want to express my gratitude to the current and former members of this lab, who have really made it a great place to work.

Finally, I want to thank my family and Kurt for being the most supportive and calm people on the planet, I don't know how you do that but it is definitely in large part thanks to all of you that I have not lost my mind (yet).

Thank you! 


\section{Curriculum vitae}

Heike Siegler

born 22.04.1987 in Münster, Germany

University Education

since Doctoral thesis „Biogenesis of Lipid Bodies in Lobosphaera

Nov 2012 incisa“

University of Göttingen, Albrecht-von-Haller Institute for Plant

Sciences, Department for Plant Biochemistry

Nov 2011 External Master Thesis “Cell-specific and Inducible Expression

- May 2012 of Salt Tolerance Genes in Transgenic Rice"

Australian Centre for Plant Functional Genomics, Adelaide

Sep 2009 Student Exchange

- Sep 2010 École Polytechnique Fédérale de Lausanne und Université de Lausanne, Switzerland

Sep 2009 Master of Science: Biotechnology/Molecular Biotechnology

- May 2012 RWTH Aachen University, Germany

Final grade: 1.8

Jun 2009 Bachelor Thesis “The Impact of Different GAR22 Variants on Cell

- Aug 2009 Motility"

Helmholtz Institute for Biomedical Engineering, Department of Cell Biology

Okt 2006 Bachelor of Science: Biotechnology/Molecular Biotechnology

- Aug 2009 RWTH Aachen University, Germany

Final grade: 2.1

Publications

Gutjahr, C., Radovanovic, D., Geoffroy, J., Zhang, Q., Siegler, H., Chiapello, M., Casieri, L., An, K., An, G., Guiderdoni, E., et al. (2012). The half-size ABC transporters STR1 and STR2 are indispensable for mycorrhizal arbuscule formation in rice. Plant J. 69, 906-920.

Gutjahr, C., Siegler, H., Haga, K., Iino, M., and Paszkowski, U. (2015). Full establishment of arbuscular mycorrhizal symbiosis in rice occurs independently of enzymatic jasmonate biosynthesis. PLoS One 10, e0123422. 
